\title{
Eine Finite-Elemente-Methode für nicht-isotherme inkompressible Strömungsprobleme
}

\author{
Dissertation \\ zur Erlangung des mathematisch-naturwissenschaftlichen Doktorgrades \\ "Dr. rerum naturalium" \\ an der Georg-August-Universität Göttingen
}

vorgelegt von

Johannes Löwe

aus

Göttingen

Göttingen 2011 
D7

Referent: Prof. Dr. Gert Lube

Koreferentin: Prof. Dr. Gerlind Plonka-Hoch

Tag der mündlichen Prüfung: 14.07.2011 


\section{Inhaltsverzeichnis}

Inhaltsverzeichnis $\quad$ i

$\begin{array}{ll}\text { Einleitung } & 1\end{array}$

1 Mathematisches Modell für nicht-isotherme Strömungen 5

1.1 Strömungsmechanik . . . . . . . . . . . . . . . 5

1.2 Das Navier-Stokes-Fourier-Modell . . . . . . . . . . . . . . . . . . . . 6

1.3 Das Oberbeck-Boussinesq-Modell . . . . . . . . . . . . . . . . . 8

1.4 Randbedingungen . . . . . . . . . . . . . . . . 9

1.5 Physikalische Parameter und Entdimensionalisierung . . . . . . . . . . . . 10

1.6 Numerische Strömungsmechanik (CFD) . . . . . . . . . . . . . . . . . . 11

1.7 Notation . . . . . . . . . . . . . . . . . . . . . . . . 14

$\begin{array}{lll}2 & \text { Die räumliche Diskretisierung } & \mathbf{1 7}\end{array}$

2.1 Variationsformulierung . . . . . . . . . . . . . . . . . . . 18

2.2 Das Ritz-Galerkin-Verfahren . . . . . . . . . . . . . . . . . . . 22

2.3 Die Finite-Elemente-Methode . . . . . . . . . . . . . . . . . . . . . . . . . 24

2.3.1 Viereck- und Hexaederelemente . . . . . . . . . . . . . . . . . 25

2.3.2 Geeignete Räume für die Geschwindigkeit und den Druck . . . . . 28

2.3.3 Interpolation in $\mathcal{Q}_{k, h}$ und $\mathbf{Z}_{h} \ldots \ldots \ldots \ldots \ldots$

2.3.4 Finite-Elemente-Software . . . . . . . . . . . . . . . . 30

2.4 Divergenz-Stabilisierung . . . . . . . . . . . . . . . . . . . . . 30

2.5 Druckseparation zur Verbesserung der Diskretisierungsfehler . . . . . . . . 31

2.6 Die spektralen Eigenschaften der räumlichen Diskretisierung . . . . . . . . 32

3 Turbulenzmodellierung $\quad 35$

3.1 Die Energieverteilung auf unterschiedlichen Skalen . . . . . . . . . . . . . . 36

3.2 Ansätze zur Turbulenzmodellierung . . . . . . . . . . . . . . . . . . 39

3.3 Die Reynolds-gemittelten Gleichungen . . . . . . . . . . . . . . . 40 
3.4 Large Eddy Simulation . . . . . . . . . . . . . . . . . . . . . . . . . 43

3.4.1 Eine Betrachtung der Ursachen für die Probleme . . . . . . . . . . 43

3.4.2 Die räumliche Filterung der Modellgleichungen . . . . . . . . . . . 44

3.4.3 Das Taylor- und Rational-LES Modell . . . . . . . . . . . . . . 46

3.4.4 Das Lilly-Eidson Modell . . . . . . . . . . . . . . . . . . . 47

3.4.5 LES mittels VMS-Turbulenzmodellen . . . . . . . . . . . . . 48

3.5 Die Implementierung der SGS-Modellterme . . . . . . . . . . . . . . . 51

3.6 Zusammenfassung . . . . . . . . . . . . . . . . 52

4 Analysis zum semi-diskreten Problem $\quad 53$

4.1 Vorbereitungen ........................ 54

4.2 Eindeutigkeit und Stabilitätsabschätzungen . . . . . . . . . . . 56

4.3 A-priori Fehlerabschätzung für Geschwindigkeit und Temperatur . . . . . 59

4.4 Abgeleitete Fehlerabschätzung für den Druck . . . . . . . . . . . . . . . 66

4.5 Diskussion . . . . . . . . . . . . . . . . . . . . 68

$\begin{array}{lll}5 & \text { Die zeitliche Diskretisierung } & \mathbf{7 1}\end{array}$

5.1 Differentiell algebraische Gleichungen . . . . . . . . . . . . . . . . 72

5.2 Grundlagen der zeitlichen Diskretisierung . . . . . . . . . . . . . . . 74

5.3 BDF- und sBDF-Verfahren . . . . . . . . . . . . . . . 74

5.4 Implizite Runge-Kutta-Verfahren . . . . . . . . . . . . . . . . . . 77

5.4 .1 Äquivalente Formulierung . . . . . . . . . . . . . . . . . . . . . . . . . . 78

5.4 .2 Steif-stabile DIRK-Verfahren . . . . . . . . . . . . . . 79

5.4.3 Die Radau-IIA-Verfahren . . . . . . . . . . . . . . . . . . . 80

5.4 IMEX Runge-Kutta Verfahren . . . . . . . . . . . . . . . . 82

5.5 Stabilitätsgebiete und Zeitschrittweitenbeschränkung . . . . . . . . . . 83

5.6 Stetige Ausgabe . . . . . . . . . . . . . . . . . . . . . . . . . . . . . . . . . . . . . 85

5.7 Zusammenfassung . . . . . . . . . . . . . . . . . 85

6 Umströmung eines zweidimensionalen Zylinders $\quad \mathbf{8 7}$

6.1 Benchmarkbeschreibung . . . . . . . . . . . . . . . . 88

6.2 Benchmarkgrößen . . . . . . . . . . . . . . . . . . . 88

6.3 Diskretisierung . . . . . . . . . . . . . . . . . . . . . . . . 91

6.4 Linearisierung . . . . . . . . . . . . . . . . . . . . . 93

6.5 Konvergenzuntersuchung der zeitlichen Diskretisierung . . . . . . . . . . 94

6.6 Konvergenzuntersuchung Ort . . . . . . . . . . . . . . . . . . 98

6.7 Fazit . . . . . . . . . . . . . . . . . . . . 100 
7 Laminare nicht-isotherme Hohlraumströmung 103

7.1 Problembeschreibung . . . . . . . . . . . . . . . . . . . . . . 103

7.2 Beschreibung der Benchmarkwerte . . . . . . . . . . . . . . . . 104

7.3 Zeitabhängiger Lösungsverlauf . . . . . . . . . . . . . . . . . 106

7.4 Beschreibung wesentlicher Strömungsmerkmale der stationären Lösung . 107

7.5 Gitterkonvergenzstudie . . . . . . . . . . . . . . . . . . . 109

7.6 Druckseparation . . . . . . . . . . . . . . . . . . 113

8 Turbulente nicht-isotherme Hohlraumströmung 121

8.1 Beschreibung des Experiments . . . . . . . . . . . . . . . . . . . 121

8.2 Wahl der Filterweite . . . . . . . . . . . . . . . . . . . . . 123

8.3 Zeitliche Mittelung . . . . . . . . . . . . . . . . . . . . . . 125

8.4 Zweidimensionale Rechnungen . . . . . . . . . . . . . . . . . 125

8.5 Dreidimensionale Rechnungen . . . . . . . . . . . . . . . . . . . 128

8.6 Untersuchung des Energiespektrums . . . . . . . . . . . . . . . . 129

$\begin{array}{ll}\text { Abschlussbetrachtung } & 131\end{array}$

$\begin{array}{ll}\text { Symbolverzeichnis } & 133\end{array}$

$\begin{array}{ll}\text { Literaturverzeichnis } & \mathbf{1 3 5}\end{array}$

$\begin{array}{lr}\text { Danksagung } & 141\end{array}$ 


\section{Einleitung}

Die numerische Strömungsmechanik ist ein wichtiges Teilgebiet der angewandten Mathematik. Ihr Ziel ist die zuverlässige Vorhersage von Strömungen und deren Eigenschaften. Sie bedient sich der stetig wachsenden Rechenleistung von Computern und kann so ein Experiment oder einen Modellversuch ergänzen oder sogar ersetzen. Neben der Suche nach geeigneten mathematischen Gleichungen, dem Strömungsmodell, gehört daher auch die Entwicklung von Verfahren, mit denen diese Gleichungen von Computern effizient gelöst werden können zu diesem weiten Forschungsfeld.

Für den wichtigen Fall nicht-isothermer inkompressibler Strömungen wird bei geringen Temperaturvariationen das Oberbeck-Boussinesq-Modell zur Beschreibung des Geschwindigkeitsfeldes $\mathbf{u}$, des kinematischen Druckes $p$ und der Temperatur $\theta$ verwendet. Strömungen, die durch dieses Modell angemessen beschrieben werden können, finden sich in vielen Anwendungsbereichen wieder. Dazu gehören z.B. die Innenraumströmungen von Wohn- und Büroräumen oder auch von Flugzeugkabinen, die durch Belüftungs- und Klimaanlagen gesteuert werden können, um ein für Menschen angenehmes Raumklima zu erzeugen. Auch die Belüftung von technischen Anlagen (z.B. Rechenzentren) oder von einzelnen Geräten mit dem Ziel der geregelten Wärmeabfuhr kann durch dieses Modell beschrieben werden. Häufiges Ziel der Simulation ist es, die Effektivität oder Effizienz geplanter Anlagen im Voraus zu überprüfen und ggf. zu verbessern.

Nach einer räumlichen und zeitlichen Diskretisierung können Computerprogramme implementiert werden, die die Modellgleichungen näherungsweise lösen. Da die Strömungen in vielen praktischen Anwendungen jedoch turbulent sind und die auftretenden Strukturen sowohl räumlich als auch zeitlich ein großes Spektrum an Skalen umfassen, ist die direkte numerische Simulation turbulenter Strömungen, trotz enormer verfügbarer Rechenleistungen, nur in wenigen Spezialfällen möglich. Die Modellgleichungen müssen daher um ein Turbulenzmodell ergänzt werden, das die Anforderungen an die räumliche und zeitliche Auflösung der Diskretisierung reduziert und dabei trotzdem sicherstellt, dass die aufgelösten energiereichen Skalen korrekt simuliert werden. Nur die Entwicklung von Turbulenzmodellen hat es überhaupt möglich gemacht, dass mit der heute verfügbaren Rechenleistung von Rechenzentren turbulente Strömungssimulationen in praktischen Anwendungen möglich sind. 


\section{Ziele dieser Arbeit}

Ziel dieser Arbeit war die Entwicklung eines Strömungslösers für nicht-isotherme inkompressible Strömungen auf der Grundlage des Oberbeck-Boussinesq-Modells. Dabei sollten in allen Bereichen mathematisch fundierte Verfahren verwendet werden, die sich modular implementieren lassen.

Bei der räumlichen Diskretisierung wurde eine stabile Diskretisierung mittels TaylorHood-Elementen gewählt, die eine zusätzliche Stabilisierung des Drucks überflüssig macht. So müssen an dieser Stelle keine Stabilisierungsparameter gewählt bzw. optimiert werden. Ein zentraler Punkt dieser Arbeit ist die erstmalige Verwendung eines Variationellen-Multiskalen-Diffusionsterms zur Turbulenzmodellierung für nichtisotherme Probleme. Diese Art der Turbulenzmodellierung geht auf eine Arbeit von Hughes [33] zurück und orientiert sich gleichzeitig am weit verbreiteten SmagorinskyTurbulenzmodell. Eine Fehleranalysis dieser Methode für den isothermen Fall wurde in [38] und [63] durchgeführt. Diese wird in der vorliegenden Arbeit um den nichtisothermen Fall erweitert.

Zur vollständigen Implementierung eines Strömungslösers wird auch ein Zeitschrittverfahren benötigt. Neben einer Betrachtung klassischer Runge-Kutta- und Mehrschrittverfahren werden auch IMEX-Varianten (implizit/explizit) dieser Verfahren aus [4] und [5] vorgestellt und eine Modifikation zur Verbesserung der Stabilitätseigenschaften dieser Verfahren beschrieben.

Die Analysis dieser Arbeit wird durch numerische Tests ergänzt. Dazu wurden die beschriebenen Bausteine eines Strömungslösers auf der Basis einer Finite-ElementeBibliothek (deal.II) implementiert. Die Testprobleme wurden ausgewählt, um möglichst alle Teile des Strömungslösers zu testen. Zu den Testproblemen gehört die isotherme Umströmung eines Zylinders, wobei es sich um ein verbreitetes zeitabhängiges Benchmarkproblem handelt, für das Referenzwerte aus der Literatur vorliegen. Dieses Problem stellt hohe Ansprüche an die räumliche Diskretisierung (krummlinige Ränder) und das Zeitschrittverfahren. Ferner werden nicht-isotherme Hohlraumströmungen (als Prototyp für Raumluftströmungen) im laminaren und turbulenten Fall untersucht. Hier spielt die Temperaturkopplung und eine anisotrope Gitterverfeinerung eine wichtige Rolle. Bei den turbulenten Rechnungen kommt das VMS-Turbulenzmodell zum Einsatz und die Ergebnisse werden mit experimentellen und anderen numerischen Resultaten verglichen.

\section{Aufbau der Arbeit}

In Kapitel 1 geht es zentral um das mathematische Modell, mit dem die Strömung beschrieben wird. Insbesondere werden neuere Resultate zitiert, wie das OberbeckBoussinesq-Modell systematisch aus dem allgemeineren Navier-Stokes-Fourier-Modell 
abgeleitet werden kann.

Die räumliche Diskretisierung der Modellgleichungen wird in Kapitel 2 beschrieben. Dabei werden ausführlich die Schritte von der Variationsformulierung über den GalerkinAnsatz bis zum endlichdimensionalen Anfangswertproblem nachvollzogen. Außerdem werden die speziellen mathematischen Eigenschaften der Diskretisierung mittels der Taylor-Hood-Elemente diskutiert.

Kapitel 3 beinhaltet einige Grundlagen der Turbulenzmodellierung und eine Übersicht über gängige Turbulenzmodelle. Außerdem wird ein neues Turbulenzmodell für nichtisotherme Strömungen vorgestellt und Aspekte der Implementierung besprochen.

Neben einem Eindeutigkeitsbeweis für die diskreten Lösungen des Oberbeck-BoussinesqModells mit Turbulenzmodell werden in Kapitel 4 Stabilitätsabschätzungen und eine a-priori Fehlerabschätzung bewiesen. Dabei handelt es sich um den analytischen Kern dieser Arbeit.

In Kapitel 5 werden zunächst einige Grundlagen zu differentiell-algebraischen Gleichungssystemen wiederholt und Zeitschrittverfahren aus zwei unterschiedlichen Familien vorgestellt sowie deren theoretische Eigenschaften besprochen. Auch eine mögliche Verknüpfung von zeitlicher Diskretisierung und Linearisierung der Gleichungen wird besprochen.

Anhand von numerischen Versuchen am Beispiel eines umströmten Zylinders werden in Kapitel 6 die verschiedenen Verfahren zur zeitlichen Diskretisierung aus dem vorherigen Kapitel experimentell untersucht und miteinander verglichen. In den letzten beiden Kapiteln werden Resultate numerischer Simulationen laminarer und turbulenter nichtisothermer Hohlraumströmung vorgestellt und ausgewertet. 


\section{Kapitel 1}

\section{Mathematisches Modell für nicht-isotherme Strömungen}

In diesem Kapitel geht es um die Bildung eines mathematischen Modells für nichtisotherme Strömungen. Dazu betrachten wir zunächst das allgemeine Navier-StokesFourier-Modell und anschließend das nur noch für inkompressible Fluide und kleine Temperaturunterschiede gültige Oberbeck-Boussinesq-Modell. Dieses Modell wurde erstmals 1879 zur Erklärung von Abweichungen bei der experimentellen Bestimmung der Wärmeleitfähigkeit von Gasen verwendet [56]. Nachdem das Oberbeck-BoussinesqModell zuvor nur durch formale Reihenentwicklung begründet wurde, zeigen neuere analytische Untersuchungen, dass es sich bei den Lösungen des Oberbeck-BoussinesqModells um singuläre Grenzwerte von Lösungen des Navier-Stokes-Fourier-Modells handelt [20]. Für Details zur Notation sei an dieser Stelle auf Abschnitt 1.7 dieses Kapitels verwiesen.

\subsection{Strömungsmechanik}

Bei der Strömungsmechanik handelt es sich um ein Teilgebiet der Kontinuumsmechanik, bei der vom mikroskopischen Aufbau der Materie abgesehen und angenommen wird, dass das Medium (Flüssigkeit oder Gas) als ein Kontinuum angenähert werden kann. Die Gültigkeit dieser Annahme kann theoretisch untersucht werden und hängt von einer Skalenseparation ab. Ab bestimmten kleinen Längenmaßstäben muss Materie als Verbund einzelner diskreter Teilchen betrachtet werden. Auf größeren Skalen hingegen spielt diese mikroskopische Struktur keine Rolle und das einzelne Teilchen verliert an Bedeutung. So kann unter geeigneten Bedingungen auch ein Granulat (z.B. feiner Sand) als Kontinuum modelliert werden.

In einem Gebiet $\Omega \subset \mathbb{R}^{3}$ wird ein homogenes Fluid durch die Zustandsvariablen Dichte $\varrho$, Geschwindigkeit $\mathbf{u}$ und Temperatur $\vartheta$ sowie durch die thermodynamischen Funktio- 
nen Druck $p=p(\varrho, \vartheta)$, spezifische innere Energie $e=e(\varrho, \vartheta)$ und spezifische Entropie $s=s(\varrho, \vartheta)$ beschrieben. Basierend auf den Annahmen zur Massenerhaltung, Energieerhaltung und Impulserhaltung, sowie auf der bereits erwähnten Kontinuumsannahme lassen sich Evolutionsgleichungen für die zeitliche Entwicklung der Zustandsvariablen eines Fluids aufstellen.

Eine wichtige Eigenschaft von Fluiden ist die Art und Weise, auf welche die inneren Spannungskräfte von den Geschwindigkeitsgradienten abhängen. Ein Newtonsches Fluid (nach dem Newtonschen Elementargesetz der Zähigkeitsreibung) ist eine Flüssigkeit oder ein Gas, dessen Scherspannung (auch Schubspannung) sich proportional zur Schergeschwindigkeit verhält. Dies trifft auf die meisten gewöhnlichen Fluide (z.B. Luft und Wasser) zu und ist zugleich eines der einfachsten und am besten untersuchten Modelle, das in der Strömungsmechanik verwendet werden kann. Nicht-Newtonsche Fluide sind u.a. viskoplastische Bingham-Fluide (z.B. Zahnpasta), die erst ab einer bestimmten Scherspannung anfangen zu fließen und sich unterhalb dieser Spannung wie ein Festkörper verhalten. Ein anderes Beispiel ist eine viskoelastische Stärkelösung, die bei niedriger Scherspannung flüssig ist, aber unter großer Scherspannung elastisch wird. Nicht-Newtonsche Strömungsmodelle werden in dieser Arbeit jedoch keine Rolle spielen.

\subsection{Das Navier-Stokes-Fourier-Modell}

Ausgehend von Erhaltungsgleichungen lassen sich für die Dichte $\varrho=\varrho(t)$, die Geschwindigkeit $\mathbf{u}=\mathbf{u}(t)$ und die absolute Temperatur $\vartheta=\vartheta(t)$ Evolutionsgleichungen in Form von partiellen Differentialgleichungen herleiten. Eine ausführliche Darstellung der Herleitung ist in Kapitel 1 von Ref. [20] zu finden. Nach einer entsprechenden Entdimensionalisierung, bei der die dimensionsbehafteten Größen durch Referenzwerte normiert werden, erhalten wir das Navier-Stokes-Fourier-Modell (1.1) bis (1.8) (siehe [20], Seite 129). Für physikalische Größen folgen wir der Notation in [34].

\section{Das Navier-Stokes-Fourier-Modell:}

$$
\begin{gathered}
\operatorname{Sr} \partial_{t} \varrho+\operatorname{div}(\varrho \mathbf{u})=0 \\
\operatorname{Sr} \partial_{t}(\varrho \mathbf{u})+\operatorname{div}(\varrho \mathbf{u} \otimes \mathbf{u})+\frac{1}{\mathrm{Ma}^{2}} \nabla p(\varrho, \vartheta)=\frac{1}{\operatorname{Re}} \operatorname{div} \mathbb{S}-\frac{1}{\mathrm{Fr}^{2}} \varrho \mathbf{f} \\
\operatorname{Sr} \partial_{t}(\varrho s(\varrho, \vartheta))+\operatorname{div}(\varrho s(\varrho, \vartheta) \mathbf{u})+\frac{1}{\mathrm{Pe}} \operatorname{div}\left(\frac{\mathbf{q}}{\vartheta}\right)=\sigma+\operatorname{Hr} \varrho \frac{Q}{\vartheta} \\
\operatorname{Sr} \partial_{t} \int_{\Omega}\left(\frac{\mathrm{Ma}^{2}}{2} \varrho|\mathbf{u}|^{2}+\varrho e(\varrho, \vartheta)\right)=\int_{\Omega}\left(\frac{\mathrm{Ma}^{2}}{\mathrm{Fr}^{2}} \varrho \mathbf{f} \cdot \mathbf{u}\right) .
\end{gathered}
$$

Der Druck $p=p(\varrho, \vartheta)$, die spezifische Entropie $s=s(\varrho, \vartheta)$ und die spezifische innere Energie $e=e(\varrho, \vartheta)$ sind differenzierbare Funktionen von $\varrho$ und $\vartheta$, die durch die GibbsRelation

$$
\vartheta D s(\varrho, \vartheta)=D e(\varrho, \vartheta)+p(\varrho, \vartheta) D\left(\frac{1}{\varrho}\right)
$$


in Zusammenhang stehen. Für ideale Gase wird $p(\varrho, \vartheta)=R \varrho \vartheta$ und $\frac{2}{3} \varrho e(\varrho, \vartheta)=p(\varrho, \vartheta)$ mit der spezifischen Gaskonstanten $R$ verwendet. Daraus ergibt sich, dass die spezifische innere Energie $s=s(\vartheta)$ allein von der Temperatur des Gases abhängt. Der viskose Spannungstensor $\mathbb{S}$ ist für Newtonsche Fluide durch

$$
\mathbb{S}=\mu(\vartheta)\left(\nabla \mathbf{u}+(\nabla \mathbf{u})^{\top}-\frac{2}{3} \operatorname{div}(\mathbf{u}) \mathbb{I}\right)+\zeta(\vartheta) \operatorname{div}(\mathbf{u}) \mathbb{I}
$$

gegeben und enthält die temperaturabhängige dynamische Viskosität $\mu(\vartheta)$ und die Volumenviskosität $\zeta(\vartheta)$. Der Wärmestrom q mit temperaturabhängiger Wärmeleitfähigkeit $k(\vartheta)$ ist gegeben durch

$$
\mathbf{q}=-k(\vartheta) \nabla \vartheta
$$

Die Entropieproduktionsrate $\sigma$ ist definiert als

$$
\sigma=\frac{1}{\vartheta}\left(\frac{\mathrm{Ma}^{2}}{\mathrm{Re}} \mathbb{S}: \nabla \mathbf{u}-\frac{1}{\mathrm{Pe}} \frac{\mathbf{q} \cdot \nabla \vartheta}{\vartheta}\right)
$$

Im Modell treten die vier physikalischen Einheiten Zeit, Länge, Masse und Temperatur auf. Es können die charakteristischen Größen für Zeit $T_{\text {ref }}$, Länge $L_{\text {ref }}$, Dichte $\varrho_{\text {ref }}$, Temperatur $\vartheta_{\text {ref }}$ und Geschwindigkeit $U_{\text {ref }}$ sowie die zusamengesetzten Größen $p_{\text {ref }}, e_{\text {ref }}, \mu_{\text {ref }}, \zeta_{\text {ref }}, k_{\text {ref }}$ und Quellterm $f_{\text {ref }}$ festgelegt werden. Alle im Modell auftretenden abhängigen und unabhängigen Variablen wurden mit diesen charakteristischen Größen skaliert. Diese Substitution ist Ursache für das Auftreten der dimensionslosen Skalierungsparameter, die in Tabelle 1.1 angegeben sind. Eine Existenzaussage für schwache Lösungen des Navier-Stokes-Fourier-Modells liefert Theorem 3.1 in [20].

\begin{tabular}{ccl}
\hline Symbol & Definition & Bezeichnung \\
\hline Sr & $L_{\text {ref }} /\left(T_{\text {ref }} U_{\text {ref }}\right)$ & Strouhal-Zahl \\
Ma & $U_{\text {ref }} / \sqrt{p_{\text {ref }} \varrho_{\text {ref }}}$ & Mach-Zahl \\
Re & $\varrho_{\text {ref }} U_{\text {ref }} L_{\text {ref }} / \mu_{\text {ref }}$ & Reynolds-Zahl \\
Fr & $U_{\text {ref }} / \sqrt{L_{\text {ref }} f_{\text {ref }}}$ & Froude-Zahl \\
Pe & $p_{\text {ref }} L_{\text {ref }} U_{\text {ref }} /\left(\vartheta_{\text {ref }} k_{\text {ref }}\right)$ & Péclet-Zahl \\
Hr & $\varrho_{\text {ref }} Q_{\text {ref }} L_{\text {ref }} /\left(p_{\text {ref }} U_{\text {ref }}\right)$ & 'Heat release parameter' \\
\hline
\end{tabular}

Tabelle 1.1: Skalierungsparameter des Navier-Stokes-Fourier-Modells.

Oft handelt es sich bei dem Beschleunigungsvektor $\mathbf{f}$ um den Gradienten eines skalaren Potentials. Im Fall von Gravitationsbeschleunigung in einem lokalen Koordinatensystem hat $\mathbf{f}$ die einfache Gestalt

$$
\mathbf{f}=\nabla F=\nabla x_{3}=(0,0,1)^{\top}
$$

und entspricht einer konstanten nach unten gerichteten Beschleunigung. 


\subsection{Das Oberbeck-Boussinesq-Modell}

In vielen Anwendungen ist die Mach-Zahl klein, d.h., die auftretenden Geschwindigkeiten sind klein im Vergleich zur Schallgeschwindigkeit. In diesem Bereich hat die Ausbreitung von Schallwellen vernachlässigbaren Einfluss auf die Strömung. Insbesondere treten keine Schockwellen oder Stöße im Fluid auf.

Sind zusätzlich die Temperaturunterschiede gering, kann die Dichte des Fluids als konstant angenommen werden. Aus (1.1) folgt dann die Inkompressibilität $\nabla \cdot \mathbf{u}=0$ des Fluids. Bei kleiner Froude-Zahl Fr $\approx$ Ma wirken sich die Temperaturunterschiede und die daraus resultierenden Dichtevariationen im wesentlichen nur noch im Auftriebsterm auf der rechten Seite der Impulsgleichung auf das Fluid aus. Unter diesen Bedingungen kommt es zu starker Schichtung innerhalb des Fluids, bei der wesentliche Eigenschaften des Fluids nur noch von der vertikalen Raumkoordinate abhängen. Bei nicht ganz so kleiner Froude-Zahl $\mathrm{Fr} \approx \sqrt{\mathrm{Ma}}$ kommt es zwar zur Schichtung, diese hat jedoch kaum Auswirkungen auf die Dichte und den Druck.

Bemerkung 1.1 Bei Simulationen mit großer räumlicher Ausdehnung (z.B. Luftströmungen in der Atmosphäre) bzw. langen Zeitskalen kann neben der Gravitationskraft auch die Corioliskraft eine wichtige Rolle spielen. Der Einfluss der Corioliskraft auf die Strömung konnte z.B. bei Experimenten zur Rayleigh-Bénard-Konvektion nachgewiesen werden [12].

Für den beschriebenen Fall niedriger Mach-Zahl und kleiner Temperaturunterschiede wird das erstmals in [56] vorgestellte Oberbeck-Boussinesq-Modell verwendet. Dabei handelt es sich um Gleichungen für das inkompressible Geschwindigkeitsfeld $\mathbf{u}$, die Temperatur $\theta$ und den Druck $P$. Die Temperatur ist jetzt nicht mehr absolut, sondern als Abweichung von einer mittleren Temperatur $\bar{\vartheta}$ zu verstehen. Bei dem Druck $P$ handelt es sich nicht um den thermodynamischen Druck, sondern um eine abhängige Variable einen Lagrange-Multiplikator - zur Erfüllung der Divergenzfreiheit der Geschwindigkeit.

\section{Das Oberbeck-Boussinesq-Modell:}

$$
\begin{gathered}
\bar{\varrho}\left(\partial_{t} \mathbf{u}+\operatorname{div}(\mathbf{u} \otimes \mathbf{u})\right)+\nabla P=\operatorname{div} \mathbb{S}-r \nabla F, \\
\operatorname{div} \mathbf{u}=0, \\
\varrho \bar{c} \bar{c}_{p}\left(\partial_{t} \theta+\operatorname{div}(\mathbf{u} \theta)\right)-\operatorname{div}(\bar{k} \nabla \theta)=0 .
\end{gathered}
$$

Wegen $\operatorname{div} \mathbf{u}=0$ hat der Spannungstensor gegenüber dem Navier-Stokes-Fourier-Modell die vereinfachte Form

$$
\mathbb{S}=\bar{\mu}\left(\nabla \mathbf{u}+(\nabla \mathbf{u})^{\top}\right)
$$

Im Oberbeck-Boussinesq-Modell werden die Viskosität $\bar{\mu}$, die spezifische Wärmekapazität bei konstantem Druck $\bar{c}_{p}$ sowie die Wärmeleitfähigkeit $\bar{k}$ bei konstanter Dichte $\bar{\varrho}$ und 
konstanter mittlerer Temperatur $\bar{\vartheta}$ ermittelt. Übereinstimmend mit der BoussinesqApproximation tritt die temperaturabhängige reduzierte Dichte $r=r(\theta)$ nur in der rechten Seite der Impulsgleichung auf und hat die Form

$$
r+\bar{\varrho} \bar{\beta}(\theta-\bar{\vartheta})=0,
$$

wobei $\bar{\beta}$ für den Wärmeausdehnungskoeffizienten steht. Es handelt sich dabei um eine Linearisierung der Dichte um die mittlere Temperatur $\bar{\vartheta}$. Der aus der mittleren Dichte resultierende Kraftterm $\bar{\varrho} \bar{\beta} \bar{\vartheta} \nabla F$ wird vom Druck absorbiert und hat keinen Einfluss auf das Geschwindigkeitsfeld.

Für den speziellen Fall

$$
\mathrm{Sr}=\mathrm{Re}=\mathrm{Pe}=\mathcal{O}(1), \quad \mathrm{Ma} \approx \epsilon, \quad \mathrm{Fr} \approx \sqrt{\epsilon}
$$

lässt sich unter bestimmten Bedingungen durch asymptotische Analysis rigoros zeigen, dass für $\epsilon \rightarrow 0$ die Lösung des Navier-Stokes-Fourier-Modells gegen die Lösung des Oberbeck-Boussinesq-Modells konvergieren (siehe dazu Theorem 5.2 in [20]). Diese Annahme ist für kleine Temperaturdifferenzen $(<20 K)$ und für Strömungen mit kleiner Machzahl $(<0.3)$ gerechtfertigt [29].

\subsection{Randbedingungen}

Das Navier-Stokes-Fourier- und das Oberbeck-Boussinesq-Modell müssen für berandete Gebiete noch mit Randbedingungen versehen werden. Für die asymptotische Untersuchung des Navier-Stokes-Fourier-Modells im Grenzfall kleiner Mach-Zahlen und schwacher Schichtung (1.14) wurden in [20] folgende Randbedingungen verwendet:

$$
\left.\mathbf{u} \cdot \mathbf{n}\right|_{\partial \Omega}=0, \quad \mathbb{S} \mathbf{n} \times\left.\mathbf{n}\right|_{\partial \Omega}=0,\left.\quad \mathbf{q} \cdot \mathbf{n}\right|_{\partial \Omega}=0 .
$$

Diese entsprechen einer vollständigen energetischen Isolation des Systems. Das Fluid kann den Rand des Gebiets $\Omega$ nicht durchdringen, es wirkt keine Haftreibung auf das Fluid und der Wärmestrom in wandnormale Richtung verschwindet. Unter diesen Bedingungen entstehen keine Grenzschichten am Rand des Gebiets, die die analytische Untersuchung erschweren.

Eine physikalisch realistischere Randbedingung ist die Haftbedingung auf Teilen $\Gamma_{0} \subset \partial \Omega$ des Randes, bei der das Fluid an der Wand ruht. Dazu kann die Wandtemperatur oder ein fester Wärmestrom vorgeschrieben werden

$$
\left.\mathbf{u}\right|_{\Gamma_{0}}=0,\left.\quad \theta\right|_{\Gamma_{0}}=\theta_{\text {wall }} \text { oder }\left.\mathbf{q} \cdot \mathbf{n}\right|_{\Gamma_{0}}=q_{\text {wall }} .
$$

Haftbedingungen können bei kleiner Viskosität zu Grenzschichten im Geschwindigkeitsprofil führen, deren analytische Untersuchung ein eigenes Forschungsfeld ist. Durch die Verwendung von asymptotisch ermittelten Grenzschichtgesetzen können Strömungsmodelle nahe der Wand deutlich vereinfacht werden. 
Häufig kann nicht das gesamte geschlossene System modelliert werden. Dann wird das Modell auf einen Teil des Systems angewendet und es entstehen künstliche Ein- und Ausströmränder $\Gamma_{-}$und $\Gamma_{+}$die über das Skalarprodukt des Geschwindigkeitsfeldes mit dem äußeren Einheitsnormalenvektor $\mathbf{n}$ des Randes definiert werden:

$$
\Gamma_{-}:=\{x \in \partial \Omega \mid \mathbf{u} \cdot \mathbf{n}<0\}, \quad \Gamma_{+}:=\{x \in \partial \Omega \mid \mathbf{u} \cdot \mathbf{n}>0\}
$$

Diese Definitionen sind von der Lösung des Problems abhängig und können auch mit der Zeit variieren. Beim Einströmrand wird meist davon ausgegangen, dass der Zustand des Fluids von außen vorgegeben ist und es wird

$$
\left.\mathbf{u}\right|_{\Gamma_{-}}=\mathbf{u}_{\mathrm{in}},\left.\quad \theta\right|_{\Gamma_{-}}=\theta_{\text {in }}
$$

gesetzt. Am Ausströmrand soll das Fluid das Gebiet möglichst ungehindert und von der Geometrie des Randes unbeeinflusst verlassen. Insbesondere sollen auch Grenzschichten am Ausströmrand vermieden werden. Dies wird dadurch erreicht, dass die auftretenden Spannungen und der Wärmestrom gleich Null gesetzt werden:

$$
\left.(\mathbb{S}-p \mathbb{I}) \cdot \mathbf{n}\right|_{\Gamma_{+}}=0,\left.\quad \mathbf{q} \cdot \mathbf{n}\right|_{\Gamma_{+}}=0 .
$$

Dabei ist darauf zu achten, dass $p$ hier nur für die Normalenspannungen steht.

Je nach Anwendung und Art des Randes können auch aufwendige Modelle für Randbedingungen erforderlich sein. Zum Beispiel, falls kleine Strukturen auf dem Rand existieren, die nicht durch die Geometrie beschreiben werden können, aber dennoch einen nicht vernachlässigbaren Einfluss auf die Strömung haben. Darunter fällt die Wandrauhigkeit (z.B. bei der Rauhreifbildung an Tragflächen von Flugzeugen) oder die Vegetation bei atmosphärische Strömungen.

\subsection{Physikalische Parameter und Entdimensionalisierung}

Für thermische Strömungen spielen die in Tabelle 1.2 zusammen mit ihren physikalischen Einheiten angegebenen Parameter eine wichtige Rolle. Neben den dimensionslosen Größen aus Tabelle 1.1 werden zur Klassifikation von thermischen Strömungen auch die Prandtl-Zahl Pr, die Grashof-Zahl Gr und die Rayleigh-Zahl Ra verwendet:

$$
\operatorname{Pr}=\frac{\nu}{\alpha}, \quad \operatorname{Gr}=\frac{g \beta \Delta \theta_{\text {ref }} L_{\text {ref }}^{3}}{\nu^{2}}, \quad \operatorname{Ra}=\operatorname{Gr} \operatorname{Pr}=\frac{g \beta \Delta \theta_{\text {ref }} L_{\text {ref }}^{3} .}{\nu \alpha} .
$$

Luft hat bei Raumtemperatur etwa folgende Eigenschaften:

$$
\begin{gathered}
\nu=15.11 \times 10^{-6} \frac{\mathrm{m}^{2}}{\mathrm{~s}}, \quad \alpha=21.19 \times 10^{-6} \frac{\mathrm{m}^{2}}{\mathrm{~s}}, \quad \beta=3.43 \times 10^{-3} \frac{1}{\mathrm{~K}}, \\
\rho=1.205 \frac{\mathrm{kg}}{\mathrm{m}^{3}}, \quad c_{p}=1.005 \frac{\mathrm{kJ}}{\mathrm{kg} \mathrm{K}} .
\end{gathered}
$$




\begin{tabular}{lll}
\hline Symbol & SI-Einheit & Bezeichnung \\
\hline$\varrho$ & $\mathrm{kg} \cdot \mathrm{m}^{-3}$ & Massendichte \\
$\mu$ & $\mathrm{kg} \cdot \mathrm{m}^{-1} \cdot \mathrm{s}^{-1}$ & Viskosität \\
$\nu=\frac{\mu}{\varrho}$ & $\mathrm{m}^{2} \cdot \mathrm{s}^{-1}$ & kinematische Viskosität \\
$k$ & $\mathrm{~W} \cdot \mathrm{m}^{-1} \cdot \mathrm{K}^{-1}$ & Wärmeleitfähigkeit \\
$c_{p}$ & $\mathrm{~J} \cdot \mathrm{kg}^{-1} \cdot \mathrm{K}^{-1}$ & spezifische Wärmekapazität \\
$\alpha=\frac{k}{\varrho c_{p}}$ & $\mathrm{~m}^{2} \cdot \mathrm{s}^{-1}$ & Temperaturleitfähigkeit \\
$\beta$ & $\mathrm{K}^{-1}$ & Wärmeausdehnungskoeffizient \\
$\mathrm{g}=(0,-g)^{\top}$ & $\mathrm{m}^{2} \cdot \mathrm{s}^{-1}$ & Gravitationsbeschleunigung \\
\hline$L_{\mathrm{ref}}$ & $\mathrm{m}$ & charakteristische Länge \\
$U_{\text {ref }}$ & $\mathrm{m} \cdot \mathrm{s}^{-1}$ & charakteristische Geschwindigkeit \\
$T_{\text {ref }}$ & $\mathrm{s}$ & charakteristische Zeiteinheit \\
$\Delta \theta_{\text {ref }}$ & $\mathrm{K}$ & charakteristische Temperaturdifferenz \\
\hline
\end{tabular}

Tabelle 1.2: Relevante physikalische Parameter von Fluiden und Strömungen.

Zusammen mit der Gravitationskonstanten $g=9.81 \frac{\mathrm{m}}{\mathrm{s}^{2}}$ ergeben sich daraus

$$
\operatorname{Pr}=0.713 \quad \text { und } \quad \mathrm{Ra} \approx 10^{8} \frac{1}{\mathrm{~K} \mathrm{~m}^{3}} \cdot \Delta \theta_{\text {ref }} L_{\text {ref }}^{3} .
$$

Typische Raumluftströmungen erreichen bei $L_{\text {ref }} \approx 3 \mathrm{~m}$ und $\Delta \theta_{\text {ref }} \approx 10 \mathrm{~K}$ somit Rayleigh-Zahlen im Bereich $10^{10} \leq \mathrm{Ra} \leq 10^{11}$.

\subsection{Numerische Strömungsmechanik (CFD)}

Im Folgenden stellt sich die Frage, wie die hergeleiteten Gleichungen zu lösen sind. Trotz aller Vereinfachungen lassen sie sich nur in wenigen Ausnahmen exakt lösen. In vielen Bereichen (z.B. im Flugzeug- und Automobilbau, beim Entwurf von Belüftungsanlagen aller Art oder bei der Klima- und Wettervorhersage) besteht jedoch großes Interesse an der Simulation und Vorhersage von Strömungen, um den Entwicklungsprozess zu beschleunigen.

Das älteste Werkzeug zur Vorhersage von Strömungen ist das Experiment, das jedoch oft nicht im Originalmaßstab durchführbar ist. Der Übergang zum verkleinerten Modell bereitet häufig technische Probleme und erfordert seinerseits großen Entwicklungsaufwand (Windkanaltechnik) und Anlagen, die teuer im Bau und Unterhalt sind. Einige Anwendungsfelder entziehen sich dem Experiment vollständig, wie z.B. die globale Klimavorhersage.

Seit der Erfindung von Computern und der rasanten Entwicklung ihrer Rechenleistung besteht der Wunsch, mit ihrer Hilfe Näherungslösungen der mathematischen Modelle 


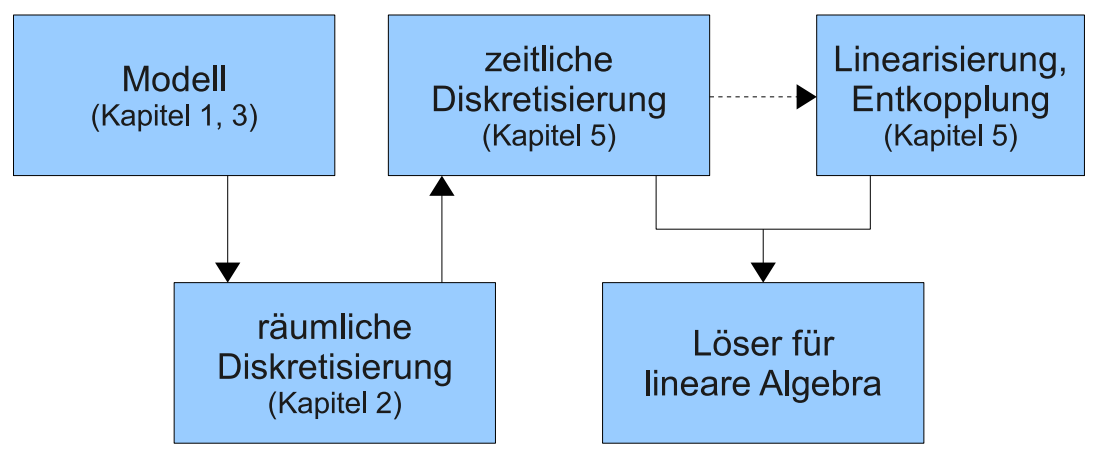

Abbildung 1.1: Gliederung des Lösungsprozesses.

zu berechnen und damit Strömungen zumindest approximiert vorherzusagen. Mit dieser Aufgabe beschäftigt sich die numerische Strömungsmechanik. Der Lösungsprozess gliedert sich dabei typischerweise in folgende Teile:

- Es wird ein Modell zur vollständigen mathematischen Beschreibung des Problems ausgewählt. In dieser Arbeit wird dazu das Oberbeck-Boussinesq-Modell verwendet, wie bereits in diesem Kapitel begründet wurde.

- Für die räumliche Diskretisierung steht eine große Auswahl an Verfahren zur Verfügung, auf die im Folgenden noch etwas genauer eingegangen werden soll. In dieser Arbeit wird eine Finite-Elemente-Diskretisierung verwendet (siehe Kapitel 2).

- Durch die räumliche Diskretisierung entstehen endlichdimensionale Anfangswertprobleme. Diese können durch implizite oder semi-implizite Zeitschrittverfahren numerisch integriert werden.

- Bei der Verwendung impliziter Zeitschrittverfahren entstehen nichtlineare Gleichungssysteme, die iterativ durch Newton- oder Picard-Iterationen gelöst werden können. Diese führen, wie auch semi-implizite Zeitschrittverfahren, auf lineare Gleichungssysteme.

- Die linearen Gleichungssysteme sind meist dünn besetzt und zu groß, um direkt gelöst werden zu können. Daher werden z.B. vorkonditionierte Krylov- oder Mehrgitterverfahren zur effizienten Lösung eingesetzt.

Bei jedem dieser Schritte muss die spezielle Struktur der Probleme berücksichtigt werden, um effiziente Verfahren zu erhalten. Insbesondere bei den beiden Schritten zur Diskretisierung in Raum und Zeit wird aufgrund der nur beschränkt zur Verfügung stehenden Rechenleistung und der daraus resultierenden niedrigen Auflösung ein nicht zu 
vernachlässigender Fehler eingeführt. Um dieses Problem zu umgehen, können die ursprünglichen Modellgleichungen um ein Turbulenzmodell erweitert werden, das die Effekte auf den nicht aufgelösten Skalen modelliert, so dass die modifizierte Lösung durch die Diskretisierung gut approximiert werden kann.

\section{Methoden zur räumlichen Diskretisierung}

Wie bereits erwähnt, steht für die räumliche Diskretisierung eine große Auswahl an Verfahren zur Verfügung, die unterschiedliche Vor- und Nachteile besitzen.

Bei der Finite-Differenzen-Methode (FDM) werden im Gebiet Punkte verteilt, an denen die Lösung approximiert werden soll. Die Differentialoperatoren werden dann durch Differenzenquotienten der Werte an diesen Punkten approximiert. Dieser Ansatz lässt sich aber nur bei regelmäßiger und äquidistanter Punkteverteilung effizient implementieren und analytisch untersuchen.

Die Finite-Volumen-Methode (FVM) entspricht einem zur FDM dualen Ansatz. Bei ihr wird das Gebiet in kleine Teilgebiete (Volumen) zerlegt und jedem Teilgebiet ein Mittelwert zugeordnet. Die Differentialoperatoren werden in Integraloperatoren überführt, so dass die Methode im Wesentlichen auf der Berechnung von Flüssen zwischen benachbarten Volumen basiert. Dieser Ansatz eignet sich besonders für Erhaltungsgleichungen. Da zur Berechnung der Flüsse Werte auf den Rändern der Teilgebiete interpoliert werden müssen, erreichen diese Verfahren jedoch meist nur eine niedrige Konvergenzordnung.

Auch die Finite-Elemente-Methode (FEM) basiert auf einer Zerlegung des Gebiets in Teilgebiete, auf denen die stetige Approximation als stückweise polynomiell angenommen wird. So kann mittels konformer Ansatzräume rigoros eine Variationsgleichung hergeleitet und eine Fehleranalysis betrieben werden. Die FEM ist bei der Anwendung auf elliptische und parabolische Probleme sehr robust. Eine ausführliche Betrachtung der Finite-Elemente-Methode ist in Kapitel 2 zu finden.

Eine weitere Möglichkeit zur Approximation der Lösung sind spektrale Methoden mit globalen Ansatzfunktionen (z.B. Tschebyscheff-Polynome oder Fourierreihen). Durch Spektraltransformationen, z.B. eine schnelle Fourier-Transformation (FFT), lassen sich die Differentialoperatoren effizient im Spektralbereich auswerten. Spektralmethoden eignen sich zwar besonders für sehr glatte Lösungen, bei denen exponentielle Konvergenz möglich ist, lassen sich aber nur bei einfachsten Geometrien (Quader- oder Kreisgebiete) effizient einsetzen.

\section{Aktuelle Entwicklungen im Bereich der Implementierung}

Ein immer wichtiger werdender Aspekt der numerischen Strömungssimulation ist die Implementierung der Verfahren für Computer. Ziel ist es, die zur Verfügung stehende Rechenleistung möglichst effizient zu nutzen. Dem gegenüber steht die stetig zunehmende 
Komplexität der Computersysteme.

Zwei wesentliche Trends dominieren dabei die aktuelle Entwicklung von Supercomputern. Ein Trend ist das stetige Anwachsen der Anzahl an Rechenkernen in Großrechnern. Diese liegt aktuell in der Größenordnung von $10^{5}$ Rechenkernen für einen einzelnen Großrechner (siehe www . top500 . org). Um die Rechenleistung vollständig zu nutzen, muss also jeder Teil der Rechnung auf alle Kerne aufgeteilt (parallelisiert) werden. Einige Verfahren lassen sich jedoch nicht gut parallelisieren, da der Kommunikationsaufwand zur Verteilung und Zusammenführung der Daten sehr groß sein kann. Aktuelle Entwicklungen zur Parallelisierung der für diese Arbeit verwendeten Finite-Elemente-Bibliothek deal. II sind in [32] zu finden.

Der zweite Trend geht in Richtung heterogener Rechnerarchitekturen. Die Rechenleistung einzelner Rechenknoten mit wenigen Rechenkernen (4 bis 16) lässt sich beträchtlich durch den Einsatz von speziellen Grafikprozessoren (GPUs) erweitern. Jeder dieser Grafikprozessoren enthält bis zu mehrere hundert programmierbare Recheneinheiten, die auch für allgemeine Rechenaufgaben programmiert werden können. Ihre Programmierung unterscheidet sich deutlich von der für klassische Rechenkerne und viele Algorithmen müssen für GPUs überarbeitet oder neu entwickelt werden, woraus ein eigenständiger Forschungszweig entstanden ist. Das Interesse an Algorithmen für GPUs ist auch dadurch begründet, dass einige der weltweit schnellsten Supercomputer mit GPUs ausgestattet sind (siehe www.top500.org).

Insgesamt ist zu beobachten, dass die Rechenleistung der einzelnen Rechenknoten schneller als die verfügbare Speicherbandbreite wächst, mit der die Knoten untereinander kommunizieren können. Von dieser Entwicklung profitieren besonders Verfahren mit hoher arithmetischer Intensität (dem Verhältnis von der Anzahl an arithmetischen Operationen zur dafür benötigten Speicherbandbreite) und Approximationsgüte, wozu auch die Finite-Elemente-Methode zählt. Im Gegensatz dazu wird die Geschwindigkeit von einfachen Verfahren (wie z.B. der FVM) durch die geringe Speicherbandbreite begrenzt.

\subsection{Notation}

In der gesamten Arbeit wird die im Folgenden beschriebene Notation verwendet. Vektorwertige Größen wie z.B. das Geschwindigkeitsfeld $\mathbf{u}$ werden mit kleinen fetten römischen Buchstaben, skalare Größen, wie der Druck $p$, werden mit Kleinbuchstaben bezeichnet. Für Matrizen und Operatoren werden Großbuchstaben genutzt. Für die einzelnen Geschwindigkeitskomponenten wird $\mathbf{u}=(u, v, w)^{\top}$ und für allgemeine Vektoren die Indexschreibweise $\mathbf{v}=\left(v_{1}, v_{2}, v_{3}\right)^{\top}$ verwendet. Die Raumkoordinaten des $\mathbb{R}^{d}$ sind $\mathbf{x}=\left(x_{1}, x_{2}, x_{3}\right)^{\top}=(x, y, z)^{\top}$.

Für partielle Ableitungen und die bei Strömungsproblemen auftretenden Differential- 
operatoren verwenden wir folgende Notation:

$$
\begin{gathered}
\text { Gradient: } \quad \nabla p=\left(\begin{array}{c}
\frac{\partial p}{\partial x} \\
\frac{\partial p}{\partial y} \\
\frac{\partial p}{\partial z}
\end{array}\right), \quad \nabla \mathbf{u}=\left(\begin{array}{lll}
\frac{\partial u}{\partial x} & \frac{\partial v}{\partial x} & \frac{\partial w}{\partial x} \\
\frac{\partial u}{\partial y} & \frac{\partial v}{\partial y} & \frac{\partial w}{\partial y} \\
\frac{\partial u}{\partial z} & \frac{\partial v}{\partial z} & \frac{\partial w}{\partial z}
\end{array}\right), \\
\text { Divergenz: } \quad \nabla \cdot \mathbf{u}=\frac{\partial u}{\partial x}+\frac{\partial v}{\partial y}+\frac{\partial w}{\partial z}, \quad \nabla \cdot A=\left(\begin{array}{c}
\nabla \cdot \mathbf{a}_{1} \\
\nabla \cdot \mathbf{a}_{2} \\
\nabla \cdot \mathbf{a}_{3}
\end{array}\right), \\
\text { Rotation: } \quad \nabla \times \mathbf{u}=\left(\begin{array}{c}
\frac{\partial w}{\partial y}-\frac{\partial v}{\partial z} \\
\frac{\partial u}{\partial z}-\frac{\partial w}{\partial x} \\
\frac{\partial v}{\partial x}-\frac{\partial u}{\partial y}
\end{array}\right) \\
\text { Tensorprodukt: } \left.\quad \begin{array}{ccc}
u u & u v & u w \\
v u & v v & v w \\
w u & w v & w w
\end{array}\right)
\end{gathered}
$$

Wir verwenden dabei für den Nablaoperator $\nabla$ kein Transponiert Symbol ${ }^{\top}$, obwohl dies an einigen Stellen formal nötig wäre. Zeitableitungen bezeichen wir mit

$$
\partial_{t} \mathbf{u}(t)=\frac{\mathrm{d}}{\mathrm{d} t} \mathbf{u}(t)
$$

Für ein Teilgebiet $G \subseteq \Omega$ sei $(\cdot, \cdot)_{G}$ das Standard- $L^{2}$-Skalarprodukt des $L^{2}(G)$ :

$$
(p, q)_{G}=\int_{G} p q \mathrm{~d} \mathbf{x}
$$

Im Fall $G=\Omega$ schreiben wir auch kurz $(p, q)=(p, q)_{\Omega}$. Für Vektor- oder Tensorwertige Funktionen werden die Skalarprodukte komponentenweisen addiert, ohne einen Unterschied in der Notation zu machen. 


\section{Kapitel 2}

\section{Die räumliche Diskretisierung}

In diesem Kapitel werden alle wichtigen Aspekte der räumlichen Diskretisierung des Oberbeck-Boussinesq-Modells dargestellt. Dazu wiederholen wir zunächst die Modellgleichungen in etwas vereinfachter Form. Dazu wird die Impulsgleichung (1.9) durch $\bar{\varrho}$ dividiert und auf die spezielle Kennzeichnung der mittleren physikalischen Parameter verzichtet.

$$
\begin{aligned}
\partial_{t} \mathbf{u}-\nabla \cdot(2 \nu \mathbb{D} \mathbf{u})+\nabla \cdot(\mathbf{u} \otimes \mathbf{u})+\nabla p+\beta \theta \mathbf{g}=\mathbf{f}, \\
\nabla \cdot \mathbf{u}=0, \\
\partial_{t} \theta-\nabla \cdot(\alpha \nabla \theta)+\nabla \cdot(\mathbf{u} \theta)=Q .
\end{aligned}
$$

Der Druck $p$ und die Quellterme f und $Q$ auf der rechten Seite seien passend skaliert. Der symmetrische Deformationstensor $\mathbb{D} \mathbf{u}$ ist durch

$$
\mathbb{D} \mathbf{u}:=\frac{\nabla \mathbf{u}+(\nabla \mathbf{u})^{\top}}{2}
$$

gegeben, so dass $2 \nu \mathbb{D} \mathbf{u}=\bar{\varrho}^{-1} \mathbb{S}$ gilt. Um zu einer räumlich diskreten Form des Systems partieller Differentialgleichungen zu gelangen, werden wir der Standardvorgehensweise für Finite-Elemente-Diskretisierungen folgen. Dazu werden die partiellen Differentialgleichungen zunächst in Variationsgleichungen überführt. Für diese werden wir uns dann auch mit der Frage nach geeigneten Funktionenräumen für die Lösungen beschäftigen. Durch Anwendung der Ritz-Galerkin-Methode werden die Variationsgleichungen auf endlichdimensionale Teilräume eingeschränkt, so dass die Wahl von geeigneten Teilräumen und deren Basen als letzter Schritt verbleibt. Außerdem soll auf spezielle Eigenschaften der Finite-Elemente-Diskretisierung für inkompressible Strömungsprobleme eingegangen werden. 


\subsection{Variationsformulierung}

Für die weitere Betrachtung wird die partielle Differentialgleichung zunächst in eine Variationsgleichung überführt. Dazu werden die Gleichungen (2.1) bis (2.3) mit beliebigen stationären (d.h. zeit-unabhängigen) Testfunktionen $\mathbf{v}, q, \psi$ multipliziert und über das Gebiet $\Omega$ integriert. Die entstehenden Skalarprodukte werden durch $(\cdot, \cdot)$ notiert. Durch partielle Integration werden für die beiden Diffusionsterme sowie für den Druckgradienten jeweils eine Ableitung auf die Testfunktionen übertragen.

$$
\begin{aligned}
\left(\partial_{t} \mathbf{u}(t), \mathbf{v}\right)+(2 \nu \mathbb{D} \mathbf{u}(t), \nabla \mathbf{v})+(\nabla \cdot(\mathbf{u}(t) \otimes \mathbf{u}(t)), \mathbf{v}) & \\
-(p(t), \nabla \cdot \mathbf{v})+(\beta \theta(t) \mathbf{g}, \mathbf{v}) & =(\mathbf{f}(t), \mathbf{v}), \\
(\nabla \cdot \mathbf{u}(t), q) & =0, \\
\left(\partial_{t} \theta(t), \psi\right)+(\alpha \nabla \theta(t), \nabla \psi)+((\mathbf{u}(t) \cdot \nabla) \theta(t), \psi) & =(Q(t), \psi) .
\end{aligned}
$$

An dieser Stelle wurde für alle zeitabhängigen Funktionen das Argument $t$ eingesetzt, worauf wir später verzichten werden. Es sei nochmals darauf hingewiesen, dass die Testfunktionen nicht von der Zeit abhängen. Ein Vorteil des Variationsproblems ist, dass es auch für Räume schwach differenzierbarer Funktionen wohlgestellt ist. Es können also Lösungen in einer größeren Klasse von Funktionen gesucht werden. Dabei gilt, dass starke Lösungen immer auch schwache Lösungen sind. Umgekehrt sind auch schwache Lösungen starke Lösungen, falls sie die entsprechenden Glattheitsanforderungen erfüllen.

Bisher wurden die Randintegrale, die durch die partielle Integration entstehen, nicht berücksichtigt. Dabei handelt es sich für die Impulsgleichung um

$$
\int_{\partial \Omega}((2 \nu \mathbb{D} \mathbf{u}(t)-p(t) \mathbb{I}) \cdot \mathbf{n}) \cdot \mathbf{v} \mathrm{d} \mathbf{s}
$$

und für die Temperaturgleichung um

$$
\int_{\partial \Omega} \alpha(\nabla \theta(t) \cdot \mathbf{n}) \psi \mathrm{d} \mathbf{s}
$$

Diese verschwinden, weil wir Dirichlet-Randwerte direkt in die Ansatzräume einarbeiten und die Testfunktionen auf diesen Randteilen verschwinden, oder weil wir natürliche Randbedingungen (1.18) verwenden, bei denen $(2 \nu \mathbb{D} \mathbf{u}-p \mathbb{I}) \cdot \mathbf{n}$ bzw. $\alpha \nabla \theta \cdot \mathbf{n}$ verschwinden. Andere Randbedingungen werden in dieser Arbeit nicht verwendet. Eine ausführliche Betrachtung dieser und verschiedener weiterer Randbedingungen für die Navier-StokesGleichungen ist in [70] zu finden. Für die Analysis beschränken wir uns auf den Fall homogener Dirichlet-Randbedingungen.

Da es sich um ein zeitabhängiges Problem über dem Zeitintervall $[0, T]$ handelt, müssen Anfangswerte für das Geschwindigkeitsfeld $\mathbf{u}$ und die Temperatur $\theta$ vorgegeben werden:

$$
\mathbf{u}(0)=u^{0}, \quad \theta(0)=\theta^{0} .
$$


Es kann kein Anfangswert für den Druck vorgegeben werden. Ein kompatibler Anfangsdruck kann bestimmt werden, indem der Divergenzoperator auf die Impulsgleichung angewendet wird, und das resultierende Poisson-Problem für $p(0)$ gelöst wird.

Um die Wohldefiniertheit der Variationsgleichungen sicherzustellen, werden für die Geschwindigkeit $\mathbf{u}=\mathbf{u}(t)$, den Druck $p=p(t)$ und die Temperatur $\theta=\theta(t)$ folgende Sobolew-Räume schwach differenzierbarer Funktionen verwendet:

$$
\begin{gathered}
\mathbf{u}(t), \mathbf{v} \in \mathbf{V}:=\left[H_{0}^{1}(\Omega)\right]^{d}=\left[W_{0}^{1,2}(\Omega)\right]^{d}, \quad \theta(t), \psi \in \Theta:=H_{0}^{1}(\Omega)=W_{0}^{1,2}(\Omega), \\
p(t), q \in Q:=L_{*}^{2}(\Omega)=\left\{q \in L^{2}(\Omega) \mid(q, 1)_{\Omega}=0\right\} \quad \forall t \in[0, T] .
\end{gathered}
$$

Der Druck ist durch die Gleichungen (2.4) nur bis auf eine Konstante eindeutig bestimmt und wird erst durch die Normierungsbedingung $(p, 1)_{\Omega}=\int_{\Omega} p \mathrm{~d} x=0$ eindeutig. Die homogenen Dirichlet-Randbedingungen sind durch die Verwendung von Spuroperatoren direkt in $\mathbf{V}$ und $\Theta$ eingearbeitet.

Definition 2.1 Eine Lösung $(\mathbf{u}, p, \theta):[0, T] \rightarrow \mathbf{V} \times Q \times \Theta$ der Variationsgleichungen (2.4) bis (2.6) für alle $t \in[0, T]$ mit den Anfangsbedingungen (2.7) nennen wir schwache Lösung des Oberbeck-Boussinesq-Modells.

Ein wichtiger Baustein für die Lösbarkeitstheorie gemischter Probleme ist die inf-sup Bedingung:

$$
\inf _{q \in Q \backslash\{0\} \mathbf{v} \in \mathbf{V} \backslash\{\mathbf{0}\}} \sup _{\|q\|_{Q}\|\mathbf{v}\|_{\mathbf{V}}} \frac{(q, \nabla \cdot \mathbf{v})_{\Omega}}{\| v^{\prime}} .
$$

Sie stellt die Eindeutigkeit des Drucks im Gleichungssystem sicher [11] und ist äquivalent zur Nečas-Ungleichung, für die ein einfacher Beweis in [10] zu finden ist. Es sei

$$
\mathbf{Z}:=\{\mathbf{v} \in \mathbf{V} \mid(q, \nabla \cdot \mathbf{v})=0 \forall q \in Q\}
$$

der Kern des Divergenzoperators. Dann können Lösung und Testfunktion für die Geschwindigkeit als $\mathbf{u}, \mathbf{v}:[0, T] \rightarrow \mathbf{Z}$ gewählt werden und der Druck verschwindet aus der Impulsgleichung, da $(p, \nabla \cdot \mathbf{v})=0$ für alle $p \in Q$. So ergeben sich für $(\mathbf{u}, \theta):[0, T] \rightarrow \mathbf{Z} \times \Theta$ die reduzierten Variationsgleichungen

$$
\begin{aligned}
\left(\partial_{t} \mathbf{u}, \mathbf{z}\right)+(2 \nu \mathbb{D} \mathbf{u}, \nabla \mathbf{z})+(\nabla \cdot(\mathbf{u} \otimes \mathbf{u}), \mathbf{z})+(\beta \theta \mathbf{g}, \mathbf{z}) & =(\mathbf{f}, \mathbf{z}), \\
\left(\partial_{t} \theta, \psi\right)+(\alpha \nabla \theta, \nabla \psi)+(\nabla \cdot(\mathbf{u} \theta), \psi) & =(Q, \psi)
\end{aligned}
$$

für alle $t \in[0, T]$ und beliebige Testfunktionen $(\mathbf{z}, \psi) \in \mathbf{Z} \times \Theta$. Das Argument $t$ wurde jetzt für zeit-abhängige Funktionen weggelassen. Der Druck $p$ lässt sich ähnlich wie ein kompatibler Startdruck $p^{0}$ bei Kenntnis von $\mathbf{u}$ und $\theta$ wegen der inf-sup Bedingung eindeutig aus der vollständigen Impulsgleichung (2.4) zurückgewinnen.

\section{Anmerkungen zum Diffusionsterm}

Für konstante kinematische Viskosität $\nu$ lässt sich der Diffusionsterm vereinfachen:

$$
-\nabla \cdot(2 \nu \mathbb{D} \mathbf{u})=-\nu \nabla \cdot\left(\nabla \mathbf{u}+(\nabla \mathbf{u})^{\top}\right)=-\nu \Delta \mathbf{u}-\nu \nabla(\nabla \cdot \mathbf{u}) .
$$


Der zweite Term verschwindet wegen der Divergenzfreiheit der Lösung. Übrig bleibt die häufig verwendete Form des Diffusionsterms mit dem Laplace-Operator. In der Variationsformulierung wird der Diffusionsterm dann zu

$$
(2 \nu \mathbb{D} \mathbf{u}, \nabla \mathbf{v})=\nu(\nabla \mathbf{u}, \nabla \mathbf{v})
$$

dem mit $\nu$ gewichteten Skalarprodukt des $\left[H_{0}^{1}(\Omega)\right]^{d}$. Diese Form des Diffusionsterms wird häufig für Simulationen laminarer Strömungen verwendet. Auch mit dem symmetrischen Spannungstensor lässt sich der Diffusionsterm in symmetrischer Form schreiben:

$$
\begin{aligned}
(2 \nu \mathbb{D} \mathbf{u}, \nabla \mathbf{v})=(\nu \mathbb{D} \mathbf{u}, \nabla \mathbf{v})+( & \left.(\mathbb{D} \mathbf{u})^{\top},(\nabla \mathbf{v})^{\top}\right) \\
& =(\nu \mathbb{D} \mathbf{u}, \nabla \mathbf{v})+\left(\nu \mathbb{D} \mathbf{u},(\nabla \mathbf{v})^{\top}\right)=(2 \nu \mathbb{D} \mathbf{u}, \mathbb{D} \mathbf{v}) .
\end{aligned}
$$

Bemerkung 2.1 Nach der Diskretisierung gilt i.A. nicht mehr $\nabla \cdot \mathbf{u}_{h}=0$ und somit unterscheiden sich dann selbst bei konstanter Viskosität die beiden Varianten des Diffusionsterms. Der symmetrische Spannungstensor beinhaltet einen Strafterm für die Divergenzfreiheit, den wir später jedoch ohnehin gesondert als Divergenz-Stabilisierung addieren werden.

Für die Analysis zur Fehlerabschätzung werden wir die symmetrische Form des Diffusionsterms mit dem symmetrischen Spannungstensor aus (2.12) verwenden. Aus mathematischer Sicht bleibt festzustellen, dass auf Lipschitzgebieten mit Haftbedingungen auf dem Rand oder periodischen Gebieten die Norm des Gradienten äquivalent zur Norm des Spannungstensors ist:

$$
\|\mathbb{D} \mathbf{v}\|_{0} \leq\|\nabla \mathbf{v}\|_{0} \leq C_{\text {Korn }}\|\mathbb{D} \mathbf{v}\|_{0}
$$

Der rechte Teil der Ungleichung wird auch Kornsche Ungleichung genannt.

\section{Anmerkungen zum Konvektionsterm}

Der nichtlineare Konvektionsterm wurde bisher in der konservativen Form geschrieben. Alternativ wird häufig die nicht konservative Form verwendet. Eine dritte Möglichkeit ist die Rotationsform. Diese drei Varianten sehen wie folgt aus:

$$
\begin{array}{ll}
\text { konservative Form: } & \nabla \cdot(\mathbf{u} \otimes \mathbf{u}), \\
\text { konvektive (auch nicht-konservative) Form: } & (\mathbf{u} \cdot \nabla) \mathbf{u}, \\
\text { Rotationsform: } & (\operatorname{curl} \mathbf{u}) \times \mathbf{u}+\frac{1}{2} \nabla(\mathbf{u} \cdot \mathbf{u}) .
\end{array}
$$


Zwei einfache komponentenweise Rechnungen für $i=1, \ldots, d$ zeigen

$$
\begin{aligned}
{[\nabla \cdot(\mathbf{u} \otimes \mathbf{u})]_{i}=\sum_{j=1}^{d} \frac{\partial}{\partial x_{j}}\left(u_{j} u_{i}\right) } & =\sum_{j=1}^{d} u_{j} \frac{\partial u_{i}}{\partial x_{j}}+\left(\sum_{j=1}^{d} \frac{\partial u_{j}}{\partial x_{j}}\right) u_{i} \\
& =[(\mathbf{u} \cdot \nabla) \mathbf{u}]_{i}+[(\nabla \cdot \mathbf{u}) \mathbf{u}]_{i}=[(\mathbf{u} \cdot \nabla) \mathbf{u}]_{i}
\end{aligned}
$$

und

$$
(\mathbf{v} \cdot \nabla) \mathbf{u}+(\mathbf{u} \cdot \nabla) \mathbf{v}=(\operatorname{curl} \mathbf{v}) \times \mathbf{u}+(\operatorname{curl} \mathbf{u}) \times \mathbf{v}+\nabla(\mathbf{u} \cdot \mathbf{v}) .
$$

Für hinreichend glatte Lösungen, die $\nabla \cdot \mathbf{u}=0$ exakt erfüllen, sind alle drei Formen des Konvektionsterms identisch. Nach der Diskretisierung wird die Divergenzfreiheit jedoch im Allgemeinen nur approximiert. Auf die spezielle Rolle des Divergenzfehlers beim Konvektionsterms wird weiter unten noch genauer eingegangen.

Bei Verwendung der Rotationsform wird der kinematische Druck $p$ durch den BernoulliDruck $\widetilde{p}=p+\frac{1}{2}(\mathbf{u} \cdot \mathbf{u})$ ersetzt, der die volle Dynamik von $\mathbf{u}$ erbt. Der verbleibende Term ist mit geringerem Aufwand zu berechnen, da es sich um ein Vektor-Vektor-Produkt handelt, während die anderen Konvektionsterme Matrix-Vektor-Produkte sind. Beim numerischen Vergleich schneidet die Rotationsform jedoch zunächst meist schlecht ab, da die Approximationsfehler des Bernoulli-Drucks größer sind und sich auch auf die Approximationsgüte der Geschwindigkeit auswirken. Durch Verwendung von DivergenzStabilisierung (siehe Abschnitt 2.4) kann der störende Einfluss auf den Geschwindigkeitsfehler jedoch stark reduziert werden (siehe Ref. [42]).

Bei der Untersuchung der Eigenschaften von den Navier-Stokes-Gleichungen und auch von skalaren Konvektions-Diffusions-Gleichungen wie der Temperaturgleichung spielt der Konvektionsterm eine entscheidende Rolle. Daher soll hier kurz auf den Einfluss des Konvektionsterms auf die Bilanzen von Impuls, Drehimpuls und Energie eingegangen werden. Dazu zitieren wir im Folgenden Ergebnisse aus Ref. [15].

Nur die konservative Form des Konvektionsterms erhält sowohl den linearen Impuls als auch den Drehimpuls. Die konvektive Form kann nur für bestimmte Diskretisierungen den Impuls, nie jedoch den Drehimpuls erhalten.

Zur Überprüfung der Energieerhaltung wird die Lösung $\mathbf{v}=\mathbf{u}$ in die Variationsgleichung als Testfunktion eingesetzt. Dies führt bei der Zeitableitung auf den Term $\frac{1}{2} \partial_{t}\|\mathbf{u}\|^{2}$. Eine vollständige Energieabschätzung der Lösung des Oberbeck-Boussinesq-Modells wird später in Lemma 4.10 bewiesen. Hier soll zunächst der Einfluss des Konvektionsterms auf die Energiebilanz untersucht werden.

Eine einfache Rechnung für die konvektive Form $b_{c}(\mathbf{w}, \mathbf{u}, \mathbf{v})=((\mathbf{w} \cdot \nabla) \mathbf{u}, \mathbf{v})$ des Konvektionsterms zeigt

$$
\begin{aligned}
b_{c}(\mathbf{w}, \mathbf{u}, \mathbf{u}) & =\left(\frac{1}{2}(\mathbf{u} \cdot \mathbf{u}), \nabla \cdot \mathbf{w}\right) \\
& =\left(\left[\frac{1}{2}(\mathbf{u} \cdot \mathbf{u})-M\right]-q, \nabla \cdot \mathbf{w}\right) \quad \forall q \in Q, M=\frac{1}{|\Omega|} \int_{\Omega} \frac{1}{2}(\mathbf{u} \cdot \mathbf{u}) \mathrm{d} \mathbf{x} .
\end{aligned}
$$


Für die exakte Lösung ist somit $b_{c}(\mathbf{w}, \mathbf{u}, \mathbf{u})=0$. Für eine diskrete Lösung $\mathbf{u}_{h}$ muss jedoch weiter abgeschätzt werden:

$$
b_{c}\left(\mathbf{w}_{h}, \mathbf{u}_{h}, \mathbf{u}_{h}\right)=\cdots \leq \inf _{q_{h} \in Q_{h}}\left\|\left[\frac{1}{2}\left(\mathbf{u}_{h} \cdot \mathbf{u}_{h}\right)-M_{h}\right]-q_{h}\right\|_{0}\left\|\nabla \cdot \mathbf{w}_{h}\right\|_{0} .
$$

$M_{h}$ sei dabei analog zu $M$ definiert. Die Störung der Energiebilanz durch den Konvektionsterm hängt also sowohl vom Divergenzfehler des Konvektionsfeldes $\mathbf{w}_{h}$ als auch von der Approximation des Bernoulli-Drucks durch den Druckraum $Q_{h}$ ab. Diese möglicherweise auch kleinen Fehler können sich mit der Zeit ansammeln und die Energiebilanz stark stören. Die Untersuchung der konservativen Form liefert das gleiche Resultat.

Abhilfe schafft eine schiefsymmetrische Form des Konvektionsterms. Für diese gilt $b_{s}(\mathbf{w}, \mathbf{u}, \mathbf{v})=-b_{s}(\mathbf{w}, \mathbf{v}, \mathbf{u})$ woraus $b_{s}(\mathbf{w}, \mathbf{u}, \mathbf{u})=0$ auch für $\nabla \cdot \mathbf{w} \neq 0$ folgt. Eine in jedem Fall schiefsymmetrische Form des Konvektionsterms kann durch

$$
b_{s}(\mathbf{w}, \mathbf{u}, \mathbf{v}):=\frac{1}{2}[((\mathbf{w} \cdot \nabla) \mathbf{u}, \mathbf{v})-((\mathbf{w} \cdot \nabla) \mathbf{v}, \mathbf{u})]
$$

definiert werden. Auch diese Form des Konvektionsterms erhält jedoch weder den Impuls noch den Drehimpuls. Weitere Untersuchungen zu den Eigenschaften der verschiedenen Formen des Konvektionsterms und auch zu den Erhaltungseigenschaften der Rotationsform sind in [50] und [73] zu finden.

Für den Konvektionsterm der Temperaturgleichung gilt eine völlig analog Betrachtung. Um auch dort eine energieerhaltende Form zu bekommen, definieren wir

$$
c_{s}(\mathbf{w}, \theta, \psi):=\frac{1}{2}[((\mathbf{w} \cdot \nabla) \theta, \psi)-((\mathbf{w} \cdot \nabla) \psi, \theta)] .
$$

Im Folgenden werden wir nur noch die Trilinearformen $b_{s}$ und $c_{s}$ als Konvektionsterme verwenden.

\subsection{Das Ritz-Galerkin-Verfahren}

Beim klassischen Ritz-Galerkin-Verfahren werden die Funktionenräume $\mathbf{V}, Q, \Theta$ durch endlichdimensionale Teilräume $\mathbf{V}_{h}, Q_{h}, \Theta_{h}$ ersetzt, deren Dimensionen mit gegen Null gehendem Diskretisierungsparameter $h$ gegen unendlich gehen:

$$
n_{u}:=\operatorname{dim}\left(\mathbf{V}_{h}\right) \stackrel{h \rightarrow 0}{\longrightarrow} \infty, \quad n_{p}:=\operatorname{dim}\left(Q_{h}\right) \stackrel{h \rightarrow 0}{\longrightarrow} \infty, \quad n_{\theta}:=\operatorname{dim}\left(\Theta_{h}\right) \stackrel{h \rightarrow 0}{\longrightarrow} \infty .
$$

Die diskrete Variationsgleichung für $\left(\mathbf{u}_{h}, p_{h}, \theta_{h}\right):[0, T] \rightarrow \mathbf{V}_{h} \times Q_{h} \times \Theta_{h}$ lautet dann

$$
\begin{aligned}
\left(\partial_{t} \mathbf{u}_{h}, \mathbf{v}_{h}\right)+\left(2 \nu \mathbb{D} \mathbf{u}_{h}, \nabla \mathbf{v}_{h}\right)+b_{s}\left(\mathbf{u}_{h}, \mathbf{u}_{h}, \mathbf{v}_{h}\right) & \\
-\left(p_{h}, \nabla \cdot \mathbf{v}_{h}\right)+\left(\beta \theta_{h} \mathbf{g}, \mathbf{v}_{h}\right) & =\left(\mathbf{f}, \mathbf{v}_{h}\right), \\
\left(\nabla \cdot \mathbf{u}_{h}, q_{h}\right) & =0, \\
\left(\partial_{t} \theta_{h}, \psi_{h}\right)+\left(\alpha \nabla \theta_{h}, \nabla \psi_{h}\right)+c_{s}\left(\mathbf{u}_{h}, \theta_{h}, \psi_{h}\right) & =\left(Q, \psi_{h}\right)
\end{aligned}
$$


für alle $t \in[0, T]$ und beliebige Testfunktionen $\left(\mathbf{v}_{h}, q_{h}, \psi_{h}\right) \in \mathbf{V}_{h} \times Q_{h} \times \Theta_{h}$. Wegen der Linearität der Variationsgleichungen in den Testfunktionen können diese durch Elemente einer Basis ersetzt werden. Wird auch die Lösung mittels Koeffizientenfunktionen $[0, T] \rightarrow \mathbb{R}^{\operatorname{dim}\left(\mathbf{V}_{h}\right) \times \operatorname{dim}\left(Q_{h}\right) \times \operatorname{dim}\left(\Theta_{h}\right)}$ bezüglich dieser Basis entwickelt, so entstehen endlichdimensionale nichtlineare Gleichungssysteme. Auch die diskreten Räume $\mathbf{V}_{h}, Q_{h}$ müssen eine inf-sup Bedingung

$$
\inf _{q_{h} \in Q_{h} \backslash\{0\}} \sup _{\mathbf{v}_{h} \in \mathbf{V}_{h} \backslash\{\mathbf{0}\}} \frac{\left(q_{h}, \nabla \cdot \mathbf{v}_{h}\right)_{\Omega}}{\left\|q_{h}\right\|_{Q}\left\|\mathbf{v}_{h}\right\|_{\mathbf{V}}} \geq b>0
$$

erfüllen, um die Eindeutigkeit des diskreten Drucks sicherzustellen und eine Fehlerabschätzung für diesen zu ermöglichen. Dabei darf die diskrete inf-sup Konstante $b$ nicht vom Diskretisierungsparameter $h$ abhängen. Die inf-sup Bedingung wird nicht automatisch durch eine konforme Wahl der Ansatzräume $\mathbf{V}_{h} \times Q_{h} \subset \mathbf{V} \times Q$ gesichert. Ein Beispiel dazu wird nach der Einführung von Finite-Elemente-Räumen in Abschnitt 2.3.2 gegeben.

Wir nehmen an, dass zu den endlichdimensionalen Ansatzräumen $\mathbf{V}_{h}, Q_{h}, \Theta_{h}$ mit den Dimensionen $n_{u}, n_{p}$ und $n_{\theta}$ Basen gegeben sind. Diese seien

$$
\begin{gathered}
\mathbf{V}_{h}=\operatorname{span}\left\{\varphi_{i} \mid i=1, \ldots, n_{u}\right\}, \quad Q_{h}=\operatorname{span}\left\{\phi_{j} \mid j=1, \ldots, n_{p}\right\}, \\
\Theta_{h}=\operatorname{span}\left\{\psi_{k} \mid k=1, \ldots, n_{\theta}\right\} .
\end{gathered}
$$

Wegen der Linearität der Variationsgleichungen in den Testfunktionen können diese durch die Basisfunktionen ersetzt werden, um ein äquivalentes System zu erhalten. Gesucht ist eine Lösung $\left(\mathbf{u}_{h}, p_{h}, \theta_{h}\right):[0, T] \rightarrow \mathbf{V}_{h} \times Q_{h} \times \Theta_{h}$, für die

$$
\begin{aligned}
\left(\partial_{t} \mathbf{u}_{h}, \varphi_{i}\right)+\left(2 \nu \mathbb{D} \mathbf{u}_{h}, \mathbb{D} \varphi_{i}\right)+b_{s}\left(\mathbf{u}_{h}, \mathbf{u}_{h}, \varphi_{i}\right)-\left(p_{h}, \nabla \cdot \varphi_{i}\right)+\left(\beta \theta_{h} \mathbf{g}, \varphi_{i}\right) & =\left(\mathbf{f}, \varphi_{i}\right), \\
\left(\nabla \cdot \mathbf{u}_{h}, \phi_{j}\right) & =0, \\
\left(\partial_{t} \theta_{h}, \psi_{k}\right)+\left(\alpha \nabla \theta_{h}, \nabla \psi_{k}\right)+c_{s}\left(\mathbf{u}_{h}, \theta_{h}, \psi_{k}\right) & =\left(Q, \psi_{k}\right),
\end{aligned}
$$

für alle $t \in[0, T]$ und alle Basisfunktionen $\varphi_{i}$ mit $i=1, \ldots, n_{u}, \phi_{j}$ mit $j=1, \ldots, n_{p}$ und $\psi_{k}$ mit $k=1, \ldots, n_{\theta}$ gitl. Weiterhin lässt sich auch die Lösung mittels dieser Basen schreiben:

$$
\mathbf{u}_{h}=\sum_{i=1}^{n_{u}} u_{i} \varphi_{i}, \quad p_{h}=\sum_{j=1}^{n_{p}} p_{j} \phi_{j}, \quad \theta_{h}=\sum_{k=1}^{n_{\theta}} \theta_{k} \psi_{k} .
$$

Die Koeffizientenvektoren werden wir wieder mit $\mathbf{u}_{h} \in \mathbb{R}^{n_{u}}, p_{h} \in \mathbb{R}^{n_{p}}$ und $\theta_{h} \in \mathbb{R}^{n_{\theta}}$ bezeichnen, da die jeweilige Bedeutung aus dem Kontext klar hervorgeht. Wir definieren zudem die folgenden Matrizen und Vektoren:

$$
\begin{gathered}
M_{u}:=\left[\left(\varphi_{j}, \varphi_{i}\right)_{\Omega}\right]_{i, j} \in \mathbb{R}^{n_{u} \times n_{u}}, \quad B:=\left[\left(\nabla \cdot \varphi_{i}, \phi_{j}\right)_{\Omega}\right]_{j, i} \in \mathbb{R}^{n_{u} \times n_{p}}, \\
M_{\theta}:=\left[\left(\psi_{l}, \psi_{k}\right)_{\Omega}\right]_{k, l} \in \mathbb{R}^{n_{\theta} \times n_{\theta}}, \quad C:=\left[\left(\beta \mathbf{g} \cdot \psi_{j}, \varphi_{k}\right)_{\Omega}\right]_{k, j} \in \mathbb{R}^{n_{u} \times n_{\theta}}, \\
A_{u}(\mathbf{w}):=\left[\left(2 \nu \mathbb{D} \varphi_{j}, \mathbb{D} \varphi_{i}\right)_{\Omega}+b_{s}\left(\mathbf{w}, \varphi_{j}, \varphi_{i}\right)\right]_{i, j} \in \mathbb{R}^{n_{u} \times n_{u}}, \\
A_{\theta}(\mathbf{w}):=\left[\left(\alpha \nabla \psi_{l}, \nabla \psi_{k}\right)_{\Omega}+c_{s}\left(\mathbf{w}, \psi_{l}, \psi_{k}\right)\right]_{k, l} \in \mathbb{R}^{n_{\theta} \times n_{\theta}}, \\
\hat{\mathbf{f}}(t)=\left[\left(\mathbf{f}(t), \varphi_{i}\right)_{\Omega}\right]_{i} \in \mathbb{R}^{n_{u}}, \quad \widehat{Q}(t)=\left[\left(Q(t), \psi_{k}\right)_{\Omega}\right]_{k} \in \mathbb{R}^{n_{\theta}} .
\end{gathered}
$$


Diese Matrizen können gleichzeitig als Operatoren bezüglich der gegebenen Basen mit folgenden Abbildungseigenschaften verstanden werden:

$$
\begin{gathered}
M_{u}, A_{u}: \mathbf{V}_{h} \rightarrow \mathbf{V}_{h}^{*}, \quad M_{\theta}, A_{\theta}: \Theta_{h} \rightarrow \Theta_{h}^{*}, \quad \hat{\mathbf{f}}(t) \in \mathbf{V}_{h}^{*}, \quad \widehat{Q}(t) \in \Theta_{h}^{*} \\
B: Q_{h} \rightarrow \mathbf{V}_{h}^{*}, \quad B^{\top}: \mathbf{V}_{h} \rightarrow Q_{h}^{*}, \quad C: \Theta_{h} \rightarrow \mathbf{V}_{h}^{*} .
\end{gathered}
$$

Es sind insbesondere $M_{u}$ und $M_{\theta}$ die jeweiligen symmetrisch positiv definiten Massematrizen, sowie $B$ der diskrete Gradient- bzw. $B^{\top}$ der diskrete Divergenzoperator. Aus der diskreten inf-sup Bedingung (2.20) folgt, dass $B^{T} M_{u}^{-1} B$ invertierbar ist und die Norm des inversen Operators unabhängig von $h$ beschränkt werden kann. So erhalten wir in Block-Matrix-Form das endlichdimensionale Anfangswertproblem

$$
\left(\begin{array}{ccc}
M_{u} & 0 & 0 \\
0 & M_{\theta} & 0 \\
0 & 0 & 0
\end{array}\right)\left(\begin{array}{c}
\mathbf{u}_{h}^{\prime}(t) \\
\theta_{h}^{\prime}(t) \\
p_{h}^{\prime}(t)
\end{array}\right)=\left(\begin{array}{c}
\hat{\mathbf{f}}(t) \\
\widehat{Q}(t) \\
0
\end{array}\right)-\left(\begin{array}{ccc}
A_{u}\left(\mathbf{u}_{h}(t)\right) & C & B \\
0 & A_{\theta}\left(\mathbf{u}_{h}(t)\right) & 0 \\
B^{\top} & 0 & 0
\end{array}\right)\left(\begin{array}{c}
\mathbf{u}_{h}(t) \\
\theta_{h}(t) \\
p_{h}(t)
\end{array}\right)
$$

für $\left(\mathbf{u}_{h}, p_{h}, \theta_{h}\right):[0, T] \rightarrow \mathbb{R}^{n_{u}} \times \mathbb{R}^{n_{p}} \times \mathbb{R}^{n_{\theta}}$. Für das semi-diskrete Problem müssen die Anfangsbedingungen noch räumlich diskret formuliert werden. Dazu seien mit $\Pi_{V_{h} / \Theta_{h}}$ kompatible Projektionen auf die Ansatzräume $\mathbf{V}_{h}, \Theta_{h}$ bezeichnet. Dann gelte

$$
\mathbf{u}_{h}(0)=\mathbf{u}_{h}^{0}:=\Pi_{V_{h}}\left(\mathbf{u}^{0}\right), \quad \theta_{h}(0)=\theta_{h}^{0}:=\Pi_{\Theta_{h}}\left(\theta^{0}\right) .
$$

Die Dirichlet-Randbedingungen sind auch für das diskrete Problem in den Ansatzräumen $\mathbf{V}_{h}, \Theta_{h}$ berücksichtigt. Eine ausführliche Betrachtung der zeitlichen Diskretisierung dieses Problems ist in Kapitel 5 zu finden.

\subsection{Die Finite-Elemente-Methode}

Bisher wurden zur Diskretisierung nur allgemein endlichdimensionale Teilräume herangezogen. In diesem Abschnitt sollen diese konkret spezifiziert und wichtige Eigenschaften dieser Räume hervorgehoben werden. Die folgende Darstellung basiert in weiten Teilen auf den Seiten 95-109 in Anhang A von [24].

Da die Dimension des Systems (2.21) sehr groß werden kann, werden bei den meisten Diskretisierungen Basen für die Ansatzräume gesucht, die zu einem dünn besetzten System führen. Einträge der Matrizen, die sich aus Skalarprodukten berechnen, werden in der Regel dann Null, wenn die entsprechenden Basisfunktionen disjunkte Träger besitzen. Damit möglichst viele Einträge verschwinden, werden Basen gesucht, deren Elemente möglichst kleine Träger haben. Bei der Finite-Elemente-Methode wird dies durch die Zerlegung des Gebiets $\Omega$ in kleine Teilgebiete erreicht. Es handelt sich demnach um eine sogenannte gitterbasierte Methode. Der Träger einzelner Basisfunktionen besteht dann aus einzelnen oder wenigen benachbarten Teilgebieten. 


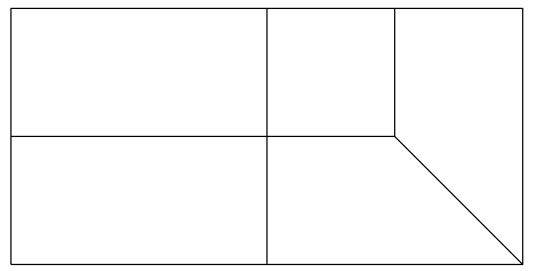

(a) zulässige Zerlegung

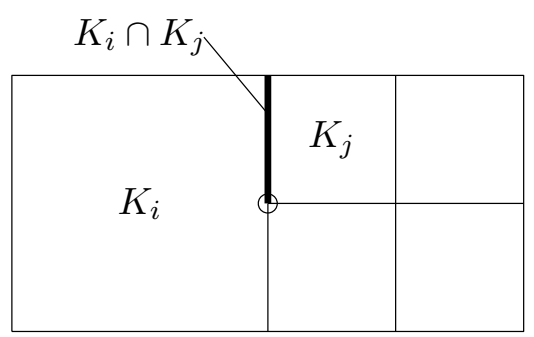

(b) unzulässige Zerlegung

Abbildung 2.1: Beispiele für eine zulässige und eine unzulässige Zerlegung.

Definition 2.2 (Triangulierung) Eine Zerlegung $\mathcal{T}=\left\{K_{i}\right\}_{i=1, \ldots, m}$ des Gebiets $\Omega$ in endlich viele abgeschlossene, polyedrische Teilgebiete $K_{i}$ mit

$$
\bar{\Omega}=\bigcup_{K \in \mathcal{T}} K
$$

wird Triangulierung genannt.

Bei der Vernetzung komplexer Geometrien oder bei dem Übergang von kleineren zu größeren Elementen kann es zu Problemen kommen. Wir werden daher die Zulässigkeit der Zerlegung weiter einschränken.

Definition 2.3 (Zulässige Zerlegung) Eine Triangulierung $\mathcal{T}$ von $\Omega$ heißt zulässig, falls der Durchschnitt zweier verschiedener $K_{i}, K_{j} \in \mathcal{T}$ leer, genau eine gemeinsame Seitenfläche, Kante oder Ecke ist.

Eine unzulässige Zerlegung ist in Abbildung 2.1(b) dargestellt, der Durchschnitt $K_{i} \cap K_{j}$ ist keine Kante von $K_{i}$. Unzulässige Zerlegungen führen zu sogenannten hängenden Knoten. Durch Hinzufügen des hängenden Knoten zu $K_{i}$ würde dieses Element zu einem degenerierten Fünfeck. Hängende Knoten erschweren die Konstruktion stetiger diskreter Funktionenräume auf solchen Gittern.

\subsubsection{Viereck- und Hexaederelemente}

Finite Elemente können auf verschiedenen Referenzzellen definiert werden. Die einfachsten Elemente sind die simplizialen Elemente (Dreiecke und Tetraeder). Als Alternative bieten sich Tensorprodukt-Elemente (Vierecke und Hexaeder) an. In drei Raumdimensionen gibt es noch weitere Mischformen wie Prismen oder Pyramiden. In kommerziellen Anwendungen werden häufig in Wandnähe strukturierte Gitter aus Hexaederoder Prismenelementen verwendet und der Rest des Gebiets mit einem unstrukturierten Tetraedergitter vernetzt. Im Übergangsbereich kommen dann Prismen- und Pyramidenelemente zum Einsatz. Im akademischen Bereich werden meist einfache Geometrien verwendet, die sich leicht vollständig durch Vierecks- oder Hexaederelemente vernetzen 


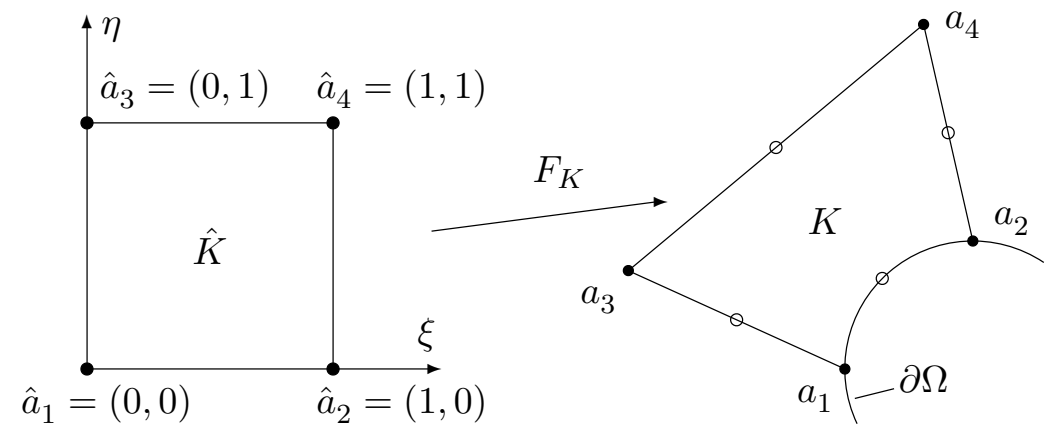

Abbildung 2.2: Referenzelement und transformiertes Element mit krummlinigem Rand.

lassen. Die Darstellung in diesem Kapitel wird sich daher auf Vierecks- und Hexaederelemente beschränken, da alle numerischen Resultate dieser Arbeit auf diesen Elementtypen basieren.

Als Referenzelement wird der n-dimensionale Einheitsquader $\hat{K}=[0,1]^{n}$ verwendet. Die Ecken von $\hat{K}$ werden mit $\hat{a}_{j}, 1 \leq j \leq 2^{n}$ bezeichnet und seien lexikographisch geordnet. Es seien $a_{j}$ die Ecken eines beliebigen konvexen Vierecks/Hexaeders $K$ in geeigneter Numerierung, dann gibt es genau eine invertierbare multilineare Abbildung $F_{K}: \hat{K} \rightarrow K$ mit:

$$
F_{K}\left(\hat{a}_{j}\right)=a_{j} \quad \text { für } 1 \leq j \leq 2^{n} .
$$

Die Abbildung $F_{K}$ ist im Allgemeinen nicht affin linear, bei einem Hexaederelement handelt es sich also nicht notwendigerweise um ein Polytop. Um krummlinige Ränder besser zu approximieren, kann die Transformation statt multilinear auch polynomiell von höherem Grad sein (siehe Abbildung 2.2).

Bisher ging es lediglich um die Zerlegung des Gebiets $\Omega$ in Teilgebiete und um deren spezielle Beschaffenheit. Im nächsten Schritt werden wir diese Zerlegung zur Definition von Funktionenräumen verwenden. Dazu benötigen wir zunächst Funktionenräume auf dem Referenzelement $\hat{K}$. Da Polynome leicht differenziert und durch numerische Quadraturformeln exakt integriert werden können, lassen sich die Systemmatrizen bei Verwendung von Polynomräumen effizient berechnen.

Definition 2.4 Für $\mathrm{x} \in \mathbb{R}^{n}$ und $\alpha \in \mathbb{N}_{0}^{n}$ sei $\mathbf{x}^{\alpha}:=\prod_{i=1}^{n} x_{i}{ }^{\alpha_{i}}$ und damit

$$
\begin{aligned}
& \mathcal{P}_{k}:=\operatorname{span}\left(\hat{K} \rightarrow \mathbb{R}, \mathbf{x} \mapsto \mathbf{x}^{\alpha} \mid \alpha \in \mathbb{N}_{0}^{d},\|\alpha\|_{1} \leq k\right), \\
& \mathcal{Q}_{k}:=\operatorname{span}\left(\hat{K} \rightarrow \mathbb{R}, \mathbf{x} \mapsto \mathbf{x}^{\alpha} \mid \alpha \in \mathbb{N}_{0}^{d},\|\alpha\|_{\infty} \leq k\right) .
\end{aligned}
$$

Wir nennen $\mathcal{P}_{k}$ den Standard-Polynomraum bzw. $\mathcal{Q}_{k}$ den Tensorprodukt-Polynomraum. Für Tensorprodukt-Elemente (Intervall, Viereck, Hexaeder) eignet sich besonders der 

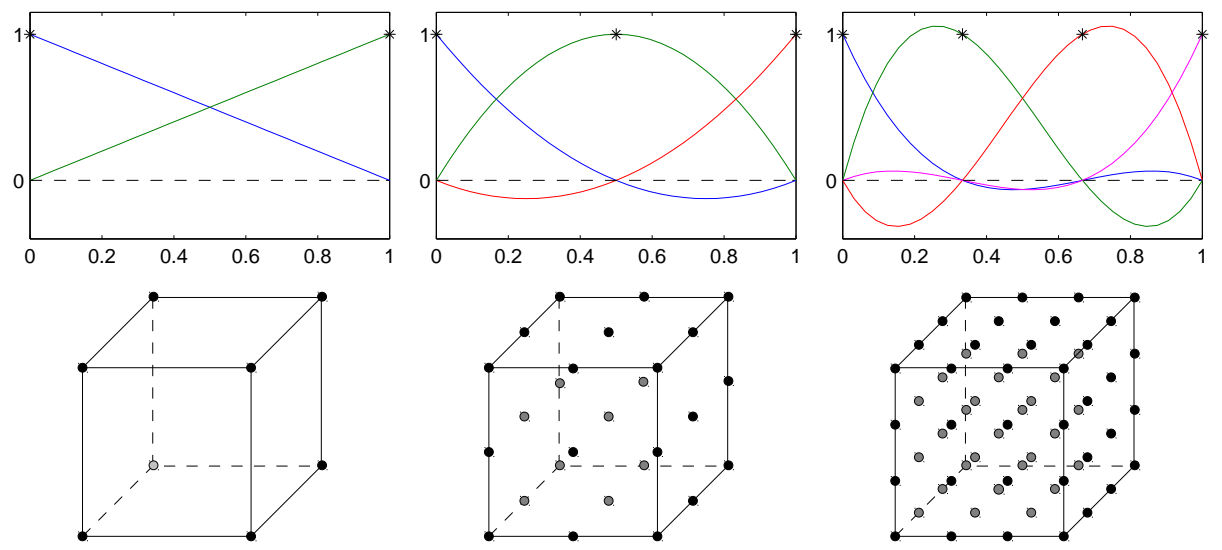

Abbildung 2.3: Eindimensionale Lagrange-Basisfunktionen mit äquidistanten Stützstellen und Verteilung der Freiheitsgrade von Lagrange-Elementen für $d=3$.

Tensorprodukt-Polynomraum. Mit dem Polynomgrad $k=1$ entspricht die Dimension, und damit die Anzahl der Basisfunktionen, genau der Anzahl der Ecken des Elements. Die gleiche Aussage gilt auch für die Paarung des Standard-Polynomraumes mit den simplizialen Elementen (Intervall, Dreieck, Tetraeder), für $d=1$ sind beide Räume identisch. Als Basis auf dem Referenzelement kann die Lagrange-Basis zu äquidistanten Stützstellen verwendet werden, bei der es sich um eine nodale Basis handelt, deren Elemente in jeweils einer Stützstelle Eins sind und in allen anderen Stützstellen verschwinden (siehe Abbildung 2.3). Die Dimension $\operatorname{dim}\left(\mathcal{Q}_{k}\right)=(k+1)^{d}$ nimmt bei wachsender Raumdimension $d$ und steigendem Polynomgrad $k$ stark zu.

Zur Definition der globalen Finite-Element-Räume auf $\Omega$ muss die Lage der Elemente berücksichtigt werden. Wir fordern, dass für jede Zelle $K$ die Transformationsabbildung $F_{K}$ verknüpft mit den auf die Zelle eingeschränkten Funktionen Element eines Polynomraumes auf dem Referenzelement ist. Dabei haben wir noch die Wahl, ob die Funktionen über die Zellkanten hinweg stetig sein sollen oder nicht.

Definition 2.5 So erhalten wir folgende Definitionen:

$$
\mathcal{P}_{k, \mathcal{T}_{h}}^{\text {disc }}:=\left\{q \in L^{2}(\Omega) \mid q_{\left.\right|_{K}} \circ F_{K} \in \mathcal{P}_{k}(\hat{K}) \quad \forall K \in \mathcal{T}_{h}\right\}
$$

und

$$
\begin{aligned}
& \mathcal{Q}_{k, \mathcal{T}_{h}}:=\left\{q \in C(\bar{\Omega}) \mid q_{\left.\right|_{K}} \circ F_{K} \in \mathcal{Q}_{k}(\hat{K}) \quad \forall K \in \mathcal{T}_{h}\right\}, \\
& \mathcal{Q}_{k, \mathcal{T}_{h}}^{\text {disc }}:=\left\{q \in L^{2}(\Omega) \mid q_{\left.\right|_{K}} \circ F_{K} \in \mathcal{Q}_{k}(\hat{K}) \quad \forall K \in \mathcal{T}_{h}\right\} .
\end{aligned}
$$

Dabei wird anstelle von $\mathcal{T}_{h}$ meist nur $h$ geschrieben, also z.B. $\mathcal{Q}_{k, h}$. Der Zusatz disc bezeichnet Räume unstetiger Funktionen. Oft wird eine Diskretisierung auch nur als $\mathcal{Q}_{k}$-Element bezeichnet, wenn verschiedene Gitter verwendet werden. 
Die Basisfunktionen des Referenzelements lassen sich mittels $F_{K}^{-1}$ auf die Zellen transformieren und für Stützstellen, die in mehreren Zellen liegen, zu stetigen Basisfunktionen zusammensetzen. So erhalten wir eine Basis, bei deren Verwendung wie gefordert schwach besetzte Systemmatrizen entstehen.

\subsubsection{Geeignete Räume für die Geschwindigkeit und den Druck}

Eine häufige Wahl für die Diskretisierung von Geschwindigkeit und Druck sind FiniteElement-Räume mit gleichem polynomiellen Grad (equal-order Ansatz). Dieser Ansatz hat den Nachteil, dass die diskrete inf-sup Bedingung (2.20) nicht erfüllt wird und die daraus resultierenden gemischten Probleme singulär, also nicht lösbar sind. Durch das Hinzufügen von speziellen Stabilisierungstermen lässt sich die Lösbarkeit der Probleme sicherstellen. Zu diesen Verfahren gehören z.B. die residuale Druckstabilisierung (Pressure-Stabilized/Petrov-Galerkin, PSPG) oder die Druckstabilisierung durch lokale Projektion (PLPS). Eine wesentliche Schwierigkeit bei der Verwendung dieser Verfahren besteht in der Notwendigkeit der Wahl passender Stabilisierungsparameter. Zu kleine Parameter liefern meist eine schlechte Approximation des Drucks, während zu große Parameter die Einhaltung der Divergenzfreiheit stören. Die Bestimmung optimaler Parameter ist ein aktuelles Gebiet der Forschung, mit der sich viele Arbeiten befassen.

Um diese Probleme zu vermeiden, wurde für die in dieser Arbeit verwendete räumliche Diskretisierung eine Familie stabiler Raumpaare $\mathbf{V}_{h} / Q_{h}$ gewählt, die die diskrete inf-sup Bedingung (2.20) erfüllt. Es handelt sich dabei um Taylor-Hood-Elemente mit stetigen Ansatzräumen für Geschwindigkeit und Druck, die sich im polynomiellen Grad um eins unterscheiden, $\mathbf{V}_{h} / Q_{h}=\left[\mathcal{Q}_{k, h}\right]^{d} / \mathcal{Q}_{k-1, h}$. Trotz Inklusion der Ansatzräume $\mathbf{V}_{h} \times Q_{h} \subset$ $\mathbf{V} \times Q$ handelt es sich um eine nicht-konforme Methode, da

$$
\mathbf{Z}_{h}=\left\{\mathbf{v}_{h} \in \mathbf{V}_{h} \mid\left(\nabla \cdot \mathbf{v}_{h}, q_{h}\right)=0 \forall q_{h} \in Q_{h}\right\} \not \subset \mathbf{Z} .
$$

Daher ist im Allgemeinen $\left\|\nabla \cdot \mathbf{u}_{h}\right\| \neq 0$. Bei der Verwendung von stetigem Druck gilt keine lokale Massenerhaltung, Quellen und Senken können im Gebiet weit auseinander liegen. Wegen $\mathbf{1}_{\Omega} \in Q_{h}$ gilt jedoch die globale Massenerhaltung $\int_{\Omega} \nabla \cdot \mathbf{u}_{h}=0$. Die Wahl eines stetigen Raumes für die Druckvariable hat den Vorteil, dass sich z.B. für gute Vorkonditionierung leicht ein Poisson-Problem für den Druck assemblieren lässt [32].

Ein Ansatz mit unstetigem Raum $Q_{h}$ für den Druck, der zu jedem Element $K$ die charakteristische Funktion $\mathbf{1}_{K}$ enthält, erreicht eine lokale Massenerhaltung, bei der $\int_{K} \nabla \cdot \mathbf{u}_{h}=0$ für alle $K \in \mathcal{T}_{h}$ gilt, erschwert aber die Konstruktion von Hilfsproblemen für den Druck. Diskrete Ansatzräume punktweise divergenzfreier Funktionen sind in der Regel problembehaftet. Bei den unstetigen Raviart-Thomas-Elementen ist nur die Normalenkomponente des Vektorfelds entlang der Elementkanten stetig, sie liegen somit nicht in $\left[H_{1}(\Omega)\right]^{d}$. Scott-Vogelius-Elemente hingegen sind insbesondere für niedrige polynomielle Ordnung nur auf Gittern mit spezieller Makroelement-Patchstruktur inf-sup stabil (siehe [48]). 


\subsubsection{Interpolation in $\mathcal{Q}_{k, h}$ und $\mathrm{Z}_{h}$}

Da die kontinuierlichen Lösungen in Finite-Elemente-Räumen approximiert werden sollen, stellt sich die Frage nach den Interpolationseigenschaften dieser Räume. Dazu sollen spezielle Interpolationsoperatoren vorgestellt werden. Der einfachste dieser Operatoren ist der Interpolationsoperator $I_{\mathrm{SZ}}: W_{p}^{l}(\Omega) \rightarrow \mathcal{Q}_{k, h}$ von Scott und Zhang [67]. Er erfüllt die quasi-lokale Interpolationsfehlerabschätzung

$$
\left\|v-I_{\mathrm{SZ}} v\right\|_{W_{p}^{m}(K)} \lesssim h_{K}^{l-m}|v|_{W_{p}^{l}(\omega(K))} \quad \forall K \in \mathcal{T}_{h}, p \geq 1,0 \leq m \leq l \leq k+1,
$$

wobei $\omega(K)$ eine Vereinigung von bestimmten zu $K$ benachbarten Zellen ist. Für $l$ muss außerdem

$$
l \geq 1 \text { für } p=1 \quad \text { und } \quad l>\frac{1}{p} \text { sonst }
$$

gelten. Zur Interpolation von Vektorfeldern kann $I_{\mathrm{SZ}}$ komponentenweise angewendet werden. Eine spezielle Eigenschaft von $I_{\mathrm{SZ}}$ ist die Erhaltung homogener DirchletRandbedingungen, so dass wir ihn auch zur Interpolation des Geschwindigkeitsfeldes verwenden können.

Für inf-sup stabile Elemente $\mathbf{V}_{h}, Q_{h}$ spielt auch die Interpolation in den Räumen diskret divergenzfreier Funktionen $\mathbf{Z}_{h}$ eine wichtige Rolle. Dazu findet sich in der Arbeit von Girault und Scott [25] eine Modifikation $I_{\mathrm{GS}}: W_{p}^{l}(\Omega) \rightarrow \mathbf{V}_{h}$ von $I_{\mathrm{SZ}}$ zur Erhaltung der diskreten Divergenz. Dieser Interpolationsoperator erfüllt neben der Interpolationsfehlerabschätzung (2.25) und der Erhaltung von homogenen Dirichlet-Randbedingungen auch folgende Bedingung

$$
\left(\nabla \cdot\left(\mathbf{v}-I_{\mathrm{GS}} \mathbf{v}\right), q_{h}\right)_{K}=0 \quad \forall q_{h} \in Q_{h} .
$$

Er bildet also divergenzfreie Funktionen $\mathbf{z} \in \mathbf{Z}$ auf diskret divergenzfreie Funktionen $\mathbf{z}_{h} \in \mathbf{Z}_{h}$ ab.

Bemerkung 2.2 Aus der Existenz eines auch hinsichtlich der Richtungsableitungen stabilen divergenzerhaltenden Interpolationsoperators folgt zwingend auch die inf-sup Stabilität der Elemente (siehe Lemma 4.3.6 in [62]). Für nicht inf-sup stabile Elemente kann folglich kein solcher Operator existieren. Eine Fehleranalysis im Raum der diskret divergenzfreien Funktionen $\mathbf{Z}_{h}$, wie sie in Kapitel 4 vorgestellt wird, macht also auch nur für inf-sup stabile Elemente Sinn.

Da für Strömungssimulationen häufig anisotrope Gitter mit lang gestreckten Elementen entlang der Wände verwendet werden, besteht Bedarf an einem anisotropen Interpolationsoperator. Damit ist gemeint, dass die Fehlerabschätzung die unterschiedlichen Durchmesser $h_{K, i}$ in die verschiedenen Koordinatenrichtungen $i$ einer kartesischen Zelle berücksichtigt und nicht nur auf dem Gesamtdurchmesser basiert. Die Modifikation $I_{\mathrm{BLR}}$ eines anisotropen Interpolationsoperators zur Erhaltung der diskreten Divergenz ist in 
[9] zu finden. Für $I_{\mathrm{BLR}}$ gilt neben (2.26) die verfeinerte Fehlerabschätzung

$$
\left\|\mathbf{v}-I_{\mathrm{BLR}} \mathbf{v}\right\|_{L^{2}(K)}^{2}+h_{K, \min }^{2}\left|\mathbf{v}-I_{\mathrm{BLR}} \mathbf{v}\right|_{H^{1}(K)}^{2} \lesssim \frac{1}{\gamma_{K}^{2}} \sum_{|\alpha|=l} \mathbf{h}_{K}^{2 \alpha}\left\|D^{\alpha} \mathbf{v}\right\|_{L^{2}(\omega(K))}^{2},
$$

mit $0 \leq l \leq k+1$. Dabei sind

$$
h_{K, \min }=\min _{i=1, \ldots, d} h_{K, i}, \quad \mathbf{h}_{K}=\left(h_{K, 1}, \ldots, h_{K, d}\right)^{\top}
$$

und $\gamma_{K}$ lokale inf-sup Konstanten, die vom Seitenverhältnis der Zellen abhängen. Für $\mathcal{Q}_{2} / \mathcal{Q}_{1}$-Elemente gilt $\gamma_{K}=C\left(1+a_{K}^{2}\right)^{-1 / 2}$, wobei $a_{K}$ das maximale Seitenverhältnis aller Zellen in einer bestimmten Nachbarschaft von $K$ bezeichnet. Eine ausführlichere Beschreibung und Details zu den Anforderungen an die Gitter sind in [62] zu finden.

\subsubsection{Finite-Elemente-Software}

Bei der Implementierung des Lösers für das Oberbeck-Boussinesq-Modell, mit dem alle in dieser Arbeit gezeigten numerischen Simulationen berechnet wurden, wurde die Finite-Elemente-Bibliothek deal. II - A Finite Element Differential Equations Analysis Library - verwendet $[6,7]$. deal. II ist eine C++-Programmbibliothek, die unter anderem Objekte zur Gebietsbeschreibung und Gittergenerierung, zur Verwaltung von Freiheitsgraden verschiedener Finite-Elemente und zur Assemblierung der Systemmatrizen bereitstellt. Dabei wird durch die Verwendung von C++-Templates eine dimensionsunabhängige Implementierung ermöglicht. Weiterhin bietet deal. II Schnittstellen zu mehreren Lösern für die linearen Gleichungssysteme wie Trilinos, PETSc und UMFPACK, sowie die Möglichkeit, die Lösung in verschiedenen Dateiformaten (Tecplot, Vtk, Eps) zu exportieren. Für die Implementierung des Strömungslösers in dieser Arbeit wurde UMFPACK [16] zusammen mit GotoBLAS2 [27] zum Lösen der linearen Gleichungssysteme und Tecplot zur Visualisierung der Lösung verwendet. Fehler- und Konvergenzplots wurden mit MATLAB erstellt.

\subsection{Divergenz-Stabilisierung}

Das Problem der verletzten Divergenzbedingung, $\mathbf{Z}_{h} \not \subset \mathbf{Z}$, tritt auch schon bei Diskretisierungen für einfachere Probleme wie dem Stokes- oder Oseen-Problem auf. Die Approximationsgüte der Massenerhaltung ergibt sich aus der Orthogonalität $\nabla \cdot \mathbf{u}_{h} \perp Q_{h}$. Insbesondere bei der Diskretisierung mit Taylor-Hood-Elementen ist $Q_{h}$ jedoch nicht sehr reichhaltig, wodurch es zu einer starken Verletzung der Massenerhaltung kommen kann.

Um $\left\|\nabla \cdot \mathbf{u}_{h}\right\|_{0}$ stärker zu kontrollieren, kann zur Impulsgleichung der Strafterm $\nabla(\gamma \nabla \cdot \mathbf{u})$ hinzuaddiert werden. Dieser ist konsistent, d.h., er ändert die kontinuierliche Lösung nicht, und begründet den häufig verwendeten Namen Grad-Div-Stabilisierung. Bei $\gamma$ 
handelt es sich um einen nichtnegativen Stabilisierungsparameter, von dem wir annehmen, dass er zellweise konstant ist, also $\gamma_{K}=\gamma_{K} \in \mathbb{R}_{0}^{+}$. Durch partielle Integration wird aus diesem Term in der Variationsformulierung die symmetrische Bilinearform $\sum_{K \in \mathcal{T}_{h}} \gamma_{K}(\nabla \cdot \mathbf{u}, \nabla \cdot \mathbf{v})_{K}$. Wie die Analysis in Kapitel 4 zeigen wird, liefert dieser Term explizite Kontrolle über

$$
\sum_{K \in \mathcal{T}_{h}} \gamma_{K}\left\|\nabla \cdot\left(\mathbf{u}-\mathbf{u}_{h}\right)\right\|_{0, K}=\sum_{K \in \mathcal{T}_{h}} \gamma_{K}\left\|\nabla \cdot \mathbf{u}_{h}\right\|_{0, K}
$$

Während die Wahl von $\gamma_{K}$ auf die kontinuierliche Lösung keinen Einfluss hat, kann diese sich stark auf die diskrete Lösung auswirken. Für $\gamma_{K} \rightarrow \infty$ wird die punktweise Divergenzfreiheit der diskreten Lösung $\mathbf{u}_{h} \in \mathbf{V}_{h} \cap \mathbf{Z}$ erzwungen. $\mathbf{V}_{h} \cap \mathbf{Z}$ ist jedoch in der Regel nicht groß genug, um weiterhin eine Approximationseigenschaft zu erfüllen. Dieses Problem macht sich schon bei beschränkten Stabilisierungsparametern bemerkbar. Für wachsende Stabilisierungsparameter $\gamma_{K}$ steigen ab einem gewissen Punkt auch die Diskretisierungsfehler an, wie numerische Versuche in den Arbeiten $[52,57]$ zeigen. In diesen Arbeiten wird auch ein adaptives Parameterdesign für $\gamma_{K}$ vorgeschlagen, durch das eine a-priori Fehlerabschätzung optimiert wird. In dieser Arbeit werden wir uns jedoch auf eine vereinfachte auf Regularitätsannahmen basierende Wahl mit global konstantem Parameter $\gamma$ beschränken. Als praxistauglicher Wert für Taylor-Hood-Elemente hat sich in zahlreichen Versuchen dabei die Wahl $\gamma_{K}=\gamma \approx 0.3$ herausgestellt.

\subsection{Druckseparation zur Verbesserung der Diskretisie- rungsfehler}

In Kapitel 4 wird eine Fehlerabschätzung bewiesen, auf deren rechter Seite auch der Term

$$
\nu^{-1} \inf _{\lambda_{h} \in Q_{h}}\left\|p-\lambda_{h}\right\|_{0}
$$

auftaucht. Da in vielen praktischen Anwendungen $\nu$ klein - und damit $\nu^{-1}$ groß ist, spielt die Approximation des Drucks auch für den Diskretisierungsfehler des Geschwindigkeitsfeldes eine wichtige Rolle. Die Fehlerabschätzung lässt sich durch eine Abänderung der Impulsgleichung verbessern, indem eine Näherung $p_{\text {sep }}$ an den Druck $p$ eingeführt wird, so dass nur noch der reduzierte Druck $p-p_{\text {sep }}$ durch die Diskretisierung approximiert werden muss. Diese Methode der Druckseparation wird in [22] für die Navier-Stokes-Gleichungen vorgestellt. Die modifizierte Impulsgleichung lautet entsprechend:

$$
\partial_{t} \mathbf{u}-\nabla \cdot(2 \nu \mathbb{D} \mathbf{u})+(\mathbf{u} \cdot \nabla) \mathbf{u}+\nabla\left(p-p_{\text {sep }}\right)+\beta \theta \mathbf{g}=\mathbf{f}-\nabla p_{\text {sep }} .
$$

Eine Wahl von $p_{\text {sep }}$ ergibt sich aus dem Ansatz, den in $\mathbf{f}$ enthaltenen Gradienten zu verwenden (siehe Variante 3 in [22]). Da beim Oberbeck-Boussinesq-Modell auch der Auftriebsterm als Kraft in der Impulsgleichung auftritt, kann dieser Term ebenfalls bei 
der Bestimmung von $p_{\text {sep }}$ berücksichtigt werden. Dazu muss folgendes Hilfsproblem gelöst werden:

$$
-\Delta p_{\mathrm{sep}}=\nabla \cdot(\beta \theta \mathbf{g}-\mathbf{f}) \quad \text { in } \Omega, \quad \partial_{n} p_{\text {sep }}=0 \quad \text { auf } \partial \Omega, \quad(p, 1)_{\Omega}=0 .
$$

In einigen Anwendungen stellt sich eine vertikale Schichtung der Strömung mit im Wesentlichen linearem Temperaturverlauf ein, siehe dazu auch das Beispiel einer thermischen Hohlraumströmung in den Kapiteln 7 und 8. Dieser Temperaturverlauf führt zu einem - nur von der vertikalen Koordinate abhängigen - quadratischen hydrostatischen Hintergrunddruck. Einfache Versuche in Abschnitt 7.6 werden zeigen, welchen Einfluss die Subtraktion dieses Hintergrunddrucks von der Impulsgleichung auf die Diskretisierungsfehler hat.

\subsection{Die spektralen Eigenschaften der räumlichen Diskreti- sierung}

Um später die Stabilität von Zeitschrittverfahren beurteilen zu können, benötigen wir Informationen über die Eigenwerte der diskreten Differentialoperatoren. Dazu untersuchen wir das eindimensionale Testproblem

$$
\partial_{t} u=\nu \partial_{x x} u-b \partial_{x} u=: L u
$$

auf dem periodisch fortgesetzten Intervall $[0,2 \pi)$. Die Eigenwerte der ersten Ableitung $\partial_{x}$ sind $i k$ und die der zweiten Ableitung $\partial_{x x}$ sind $-k^{2}$ jeweils zu den periodischen Eigenfunktionen $x \mapsto e^{i k x}$ für alle $k \in \mathbb{N}$. Die Eigenwerte von $L$ sind also $-\nu k^{2}+i b k$.

Durch die Diskretisierung werden nur noch endlich viele dieser Eigenwerte approximiert (siehe Abbildung 2.4). Bei den Eigenwerten des Diffusionsoperators $\partial_{x x}$ fällt auf, dass die Diskretisierung mit höherer Elementordnung für kleine Wellenzahlen genauere Approximationen liefert, für große Wellenzahlen jedoch dissipativer ist. Bei genauer Betrachtung des Spektrums der diskreten Konvektionsoperatoren fällt auf, dass der Eigenwert 0 doppelt auftritt. Neben der konstanten Funktion gibt es also jeweils eine weitere Funktion, für die $\partial_{x}$ diskret verschwindet. Während die exakten Eigenwerte auf einer Parabel in der komplexen Zahlenebene liegen, liegen die approximierten Eigenwerte auf einer Ellipse, die sich im Ursprung an die Parabel anschmiegt. Der Radius der Ellipse auf der reellen Achse verhält sich wie $C_{1} \frac{\nu}{h^{2}}$, während sich der Radius auf der imaginären Achse wie $C_{2} \frac{b}{h}$ verhält. Die Konstanten $C_{1}$ und $C_{2}$ hängen, wie Abbildung 2.4 zeigt, von der Diskretisierung ab. Für quadratische Elemente ist $C_{1}=7.5$ und $C_{2}=2.12$. Das Verhältnis der beiden Radien $\operatorname{Re}_{h}=\frac{C_{2}}{C_{1}} \frac{b h}{\nu}$ nennen wir Gitter-Reynoldszahl.

Auch wenn sich diese Untersuchung nur auf das eindimensionale Testproblem (2.27) bezieht, werden wir die Ergebnisse zur Bewertung der Stabilität von Zeitschrittverfahren in Kapitel 5 verwenden. 


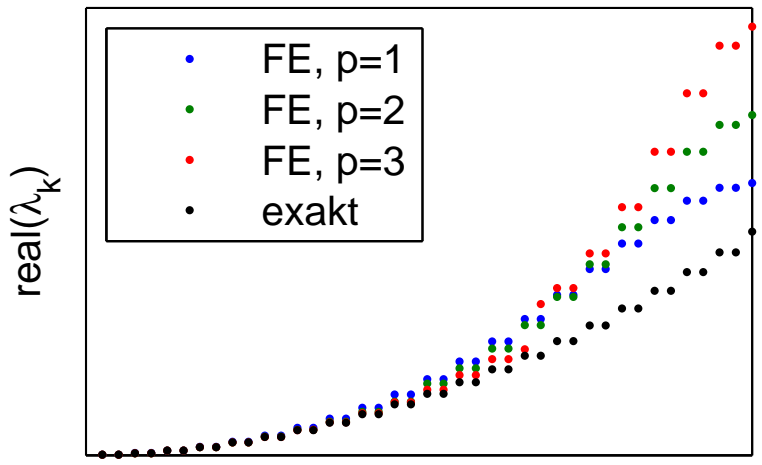

Index k

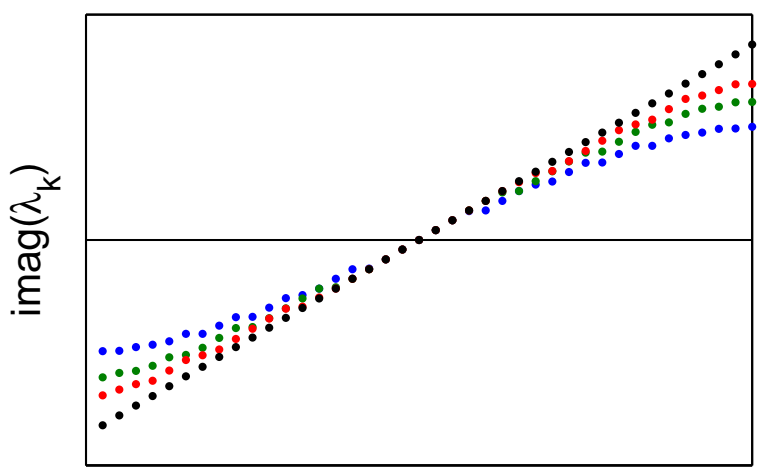

Index k

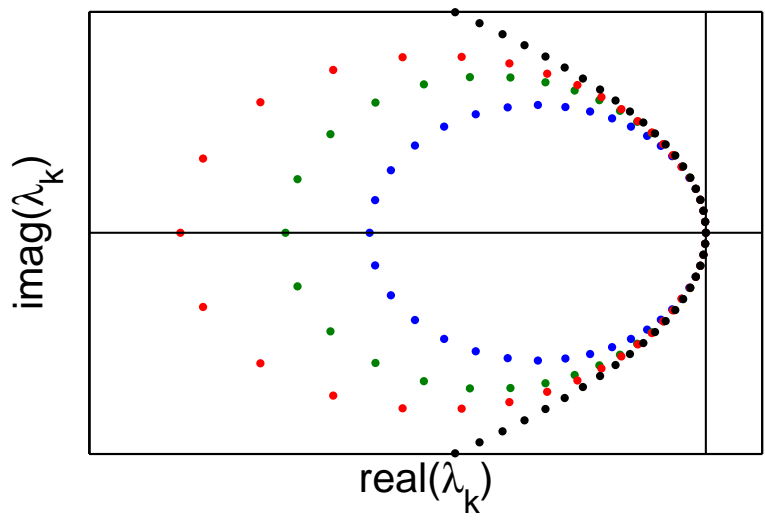

Abbildung 2.4: Exakte und approximierte Eigenwerte der Operatoren $\nu \partial_{x x}$ (oben), $b \partial_{x}$ (mitte) und $\nu \partial_{x x}+b \partial_{x}$ (unten) durch äquidistante

Finite-Elemente-Diskretisierungen der Ordnung $p=1,2,3$ mit jeweils 42 Knoten. 


\title{
Kapitel 3
}

\section{Turbulenzmodellierung}

\author{
Big whirls have little whirls \\ Which feed on their velocity; \\ And little whirls have lesser whirls, \\ And so on to viscosity.
}

- Lewis Fry Richardson (1922)

Bei der Beobachtung natürlicher Strömungen lassen sich zwei wesentlich unterschiedliche Strömungsformen erkennen. Strömungen zähflüssiger Medien bei geringer Strömungsgeschwindigkeit und räumlicher Ausdehnung sind meist laminar, d.h., das Fluid strömt in Schichten, die sich nicht vermischen. Außerdem ist die Strömung unempfindlich gegen Störungen und bei konstanten äußeren Bedingungen stellt sich meist eine stationäre Strömung ein. Das Geschwindigkeitsfeld ändert sich also nicht mit der Zeit.

Handelt es sich hingegen um ein Fluid mit geringer Viskosität und um eine Strömung mit hoher Geschwindigkeit und räumlicher Ausdehnung, so wird die Strömung anfällig gegen kleine äußere Störungen und es kommt zu Verwirbelungen auf allen Größenskalen. Diese Strömungsform zeichnet sich meist durch scheinbar zufällige dreidimensionale und instationäre (d.h. zeitabhängige) Bewegungen der Fluidteilchen aus.

Der Übergang zwischen laminarer und turbulenter Strömung lässt sich leicht an aufsteigendem Zigarettenrauch beobachten. Dieser steigt zunächst laminar in einem Strahl auf, wird dann durch äußere Störungen turbulent und vermischt sich mit der umgebenden Luft.

Erste systematische Untersuchungen zum Übergang zwischen laminaren und turbulenten Strömungen in einer Rohrleitung hat 1883 der Physiker Osborne Reynolds gemacht. Mit der nach ihm benannten dimensionslosen Reynolds-Zahl

$$
\operatorname{Re}=\frac{U L}{\nu}
$$


lässt sich das Turbulenzverhalten geometrisch ähnlicher Strömungen beschreiben. Dabei bezeichnet $U$ eine charakteristische Geschwindigkeit und $L$ eine charakteristische Länge. So verhalten sich Rohrströmungen bei gleicher Reynolds-Zahl identisch, eine Verdopplung der Strömungsgeschwindigkeit bei Halbierung des Rohrdurchmessers führt demnach zu einem identischen Strömungsverhalten. Für Rohrströmungen ermittelte Reynolds experimentell eine kritische Reynoldszahl von $R e \approx 2300$, bei deren Überschreitung die Strömung durch kleine Störungen turbulent wird. Auch bei großen Reynolds-Zahlen können Strömungen im Experiment jedoch noch laminar sein, wenn äußere Störungen sorgfältig vermieden werden.

Dieses Wissen um die Eigenschaften von Strömungen ist wichtig, da z.B. der Druckverlust in Rohrleitungen für laminare Strömungen linear von der Strömungsgeschwindigkeit abhängt, während diese Abhängigkeit bei turbulenten Strömungen quadratisch ist. Der laminare Transport ist somit deutlich energieeffizienter. Andererseits führen turbulente Strömungen zu einer besseren Durchmischung, was z.B. in chemischen Reaktoren von Vorteil ist.

In vielen praktischen Anwendungen sind die Strömungen wegen der physikalischen Parameter und den nicht vermeidbaren äußeren Störungen turbulent. Die numerische Simulation turbulenter Strömungen ist daher ein wichtiges Entwicklungswerkzeug im Automobil- und Flugzeugbau, in der Belüftungs- und Klimatechnik sowie in vielen anderen Bereichen.

Ein wesentliches Problem bei der Simulation turbulenter Strömungen ist das große Spektrum der auftretenden Längenskalen in der Strömung. Numerische Verfahren, die alle diese Längenskalen aufösen (direkte numerische Simulation, DNS), benötigen für die meisten praktischen Probleme (noch) zu viele Ressourcen in Form von Speicher und Rechenknoten und werden für einige Anwendungen mit heute denkbaren Mitteln nie möglich sein.

\subsection{Die Energieverteilung auf unterschiedlichen Skalen}

Ein wichtiger Ansatz zum besseren Verständnis von Turbulenz ergibt sich bei der Betrachtung der Verteilung von Energie $E(k)$ und Dissipation $D(k)$ auf die verschiedenen Wellenzahlen $k$, die mittels einer Fourier-Transformation des Geschwindigkeitsfeldes ermittelt werden kann. Die totale Dissipation lässt sich mittels des Dissipationsspektrums $D(k)=2 \mathrm{Re}^{-1} k^{2} E(k)$ berechnen:

$$
\partial_{t}\|\mathbf{u}\|^{2}=-\int_{0}^{\infty} D(k) \mathrm{d} k
$$

So gibt das Dissipationsspektrum Aufschluss darüber, wie sich die Dissipation über den Wellenzahlenbereich verteilt. Eine schematische Darstellung des Energie- und Dissipationsspektrums einer vollständig turbulenten Strömung zeigt Abbildung 3.1. 


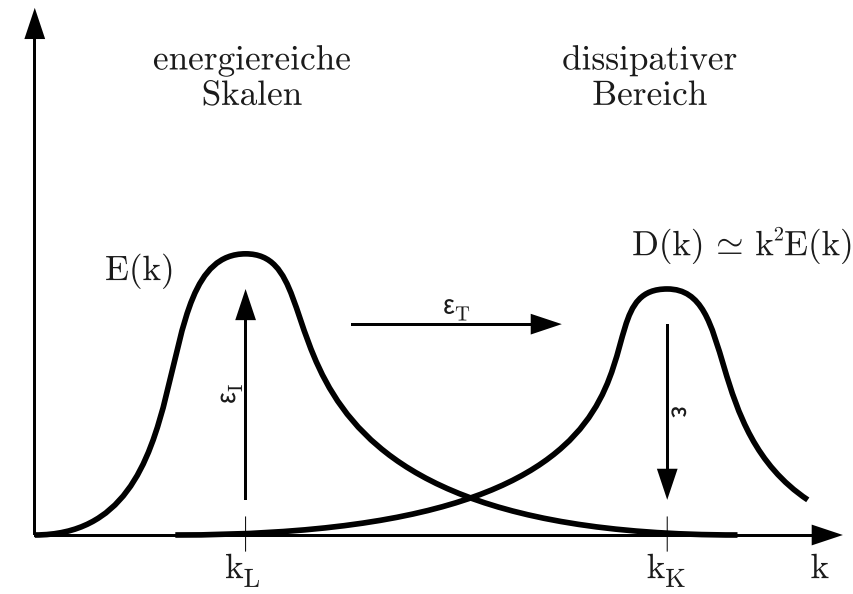

Abbildung 3.1: Energie- und Dissipationsspektrum für große Reynoldszahlen.

Die Wellenzahlen lassen sich in drei wesentliche Bereiche unterteilen. Dazu definieren wir die durchschnittliche Größe der energiereichen Wirbel $L$ und die Kolmogorov MikroSkalen $L_{K}, v_{K}$ wie folgt:

$$
L=\frac{\int_{0}^{\infty} \frac{E(k)}{k} \mathrm{~d} k}{\int_{0}^{\infty} E(k) \mathrm{d} k} \quad \text { und } \quad L_{K}=\left(\frac{\nu^{3}}{\varepsilon}\right)^{\frac{1}{4}}, \quad v_{K}=(\nu \varepsilon)^{\frac{1}{4}} .
$$

Die zugehörigen Wellenzahlen $k_{L}$ und $k_{K}$ ergeben sich als Kehrwerte der entsprechenden Längen. Auf den größten Längenskalen im Bereich von $k_{L}$ (energy containing range) wird die Strömung maßgeblich durch den äußeren Energieeintrag $\varepsilon_{I}$ und die Geometrie des Gebiets bestimmt. Dieser Längenskalenbereich beinhaltet den größten Teil der Energie und erstreckt sich bis etwa zu einem Sechstel der charakteristischen geometrischen Länge. Im Längenskalenbereich von $L_{K}$ ist die zugehörige Reynolds-Zahl $\operatorname{Re}_{K}=\frac{v_{K} L_{K}}{\nu}=1$ und die Viskosität dominiert, die Strukturen sind stabil und ihre Energie wird direkt in Wärme umgewandelt. Eine Betrachtung des Dissipationsspektrums zeigt, dass die meiste Energie in diesem Bereich dissipiert wird. Er wird daher dissipativer Bereich (dissipation range) genannt. Ordnet man den größten Skalen die Reynolds-Zahl $\operatorname{Re}_{L}$ zu, so zeigt das Verhältnis

$$
\frac{L_{K}}{L} \approx \operatorname{Re}_{L}^{-\frac{3}{4}},
$$

welche Auflösungsanforderungen eine direkte numerische Simulation für große ReynoldsZahlen erfüllen muss [64, Abschnitt 1.2.3]. In dem mittleren Skalenbereich (inertial range) $k_{L} \ll k \ll k_{K}$ muss ein Energietransfer von großen zu kleinen Skalen stattfinden. Bei den Strömungsstrukturen dieser Längenskalen handelt es sich um zufällige turbulente Fluktuationen. Diese Strukturen zerfallen immer weiter und transferieren so ihre Energie auf kleinere Skalen. Für statistisch stationäre Strömungen stellt sich ein Gleichgewicht $\varepsilon_{I}=\varepsilon_{T}=\varepsilon$ ein. 
Kolmogorov leitete unter mehreren Annahmen ein Gesetz zur Energieverteilung im mittleren Skalenbereich her. Die erste Annahme fordert lokale Homogenität und Isotropie des Strömungsfeldes [41, Definition 2]. Also auch dann, wenn das Strömungsfeld auf den energiereichen Skalen inhomogen und anisotrop ist, wird angenommen, dass diese Eigenschaften beim Zerfall der Wirbel in immer kleinere Wirbel verloren gehen und sich eine homogene und isotrope Verteilung einstellt. Außerdem werden zwei Annahmen über die zugrundeliegenden Mechanismen getroffen:

- First hypothesis of similarity: Für lokal isotrope Turbulenz ist die Verteilung der kleinsten Bewegungen universell und wird eindeutig durch die kinematische Viskosität $\nu$ und die Dissipationsrate $\varepsilon$ bestimmt.

- Second hypothesis of similarity: Für lokal isotrope Turbulenz ist die Verteilung der Bewegungen im mittleren Skalenbereich ebenfalls universell und wird allein durch die Dissipationsrate $\varepsilon$ eindeutig bestimmt.

Sehr vereinfacht resultiert daraus der Ansatz $E(k)=K_{0} \varepsilon^{x} k^{y}$ - wobei $K_{0}$ eine dimensionslose Konstante ist - mit der zugehörigen "Einheitengleichung"

$$
\left(\frac{\text { Länge }^{3}}{\text { Zeit }^{2}}\right)=\left(\frac{\text { Länge }^{2}}{\text { Zeit }^{3}}\right)^{x}\left(\frac{1}{\text { Länge }}\right)^{y},
$$

Da Länge und Zeit unabhängige Einheiten sind, bleiben als einzige konsistente Exponenten $x=\frac{2}{3}$ und $y=-\frac{5}{3}$. So erhalten wir das in vielen turbulenten Strömungen auch experimentell zu beobachtende Kolmogorov-5/3-Gesetz

$$
E(k)=K_{0} \varepsilon^{2 / 3} k^{-5 / 3} \quad \text { für } k_{L} \ll k \ll k_{K}
$$

zur Energieverteilung auf den mittleren Skalen. Abbildung 3.2 zeigt eine schematische Darstellung der Energieverteilung bei doppelt logarithmischen Achsen.

Erstmals experimentell und über einen großen Wellenzahlbereich bestätigt wurde dieses Gesetz durch Messungen der Turbulenz in Gezeitenströmungen von Grant u.a. im Jahr 1962 [28]. Für die Kolmogorov-Konstante wurde experimentell das Intervall $K_{0} \in[1.4,1.7]$ ermittelt. Wegen der Universalität der Verteilungen turbulenter Fluktuationen im mittleren Skalenbereich und darunter wird angenommen, dass diese Fluktuationen zwar wichtig für den Energietransfer in Richtung kleinerer Skalen und die anschließende Dissipation sind, dass diese aber keinen direkten Einfluss auf die großen Strukturen der Strömung haben. Dies wird auch damit begründet, dass der Energieaustausch für eine Wellenzahl $k$ hauptsächlich - aber nicht ausschließlich - im Wellenzahlbereich $[k / 2,2 k]$ stattfindet [64, Abschnitt 1.3.2]. Es besteht also die Hoffnung, dass diese universellen Fluktuationen nicht für jede Strömung berechnet werden müssen, sondern sich deren Einfluss auf die energiereichen Strömungsstrukturen modellieren lässt.

Bemerkung 3.1 Obwohl das Kolmogorov-5/3-Gesetz in vielen Experimenten bestätigt wurde, gibt es doch Kritik an der Herleitung und den zugrundeliegenden Annahmen. Eine ausführliche Betrachtung ist in [14] zu finden. Es wird z.B. angeführt, dass 


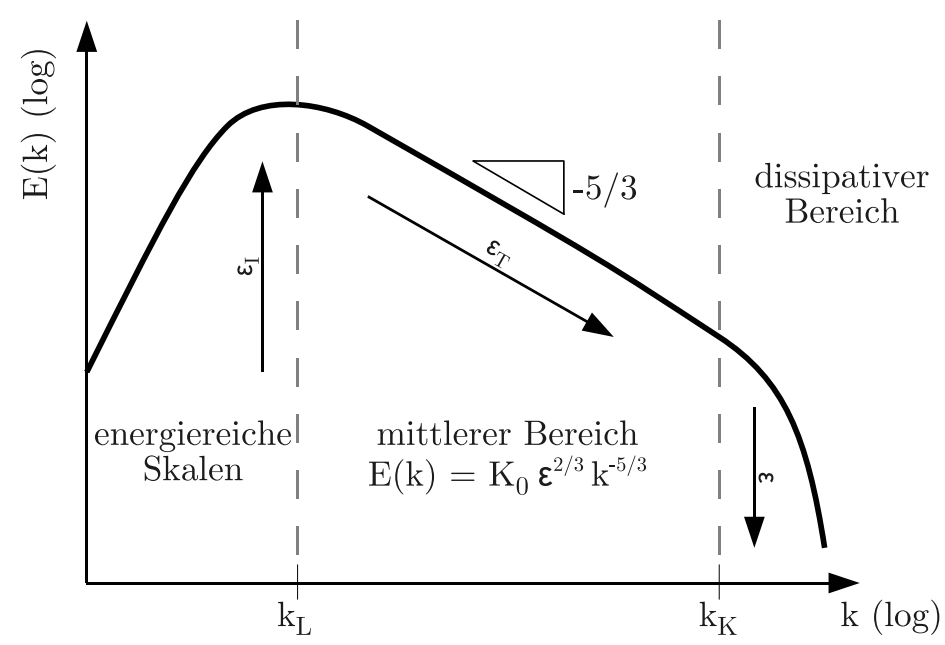

Abbildung 3.2: Energieverteilung für große Reynolds-Zahlen.

bei der Herleitung in keiner Weise die Navier-Stokes-Gleichungen als zugrundeliegendes Strömungsmodell verwendet werden und dass nicht klar ist, welchen Einfluss das Strömungsmodell auf die getroffenen Annahmen hat. Für zweidimensionale NavierStokes-Strömungen zeigen Simulationen und theoretische Überlegungen, dass das Gesetz nicht gilt. Außerdem wurde an atmosphärischen Grenzschichtströmungen mittlerweile gezeigt, dass z.B. - im Widerspruch zur ersten Annahme - Randbedingungen sehr wohl Einfluss bis weit in den mittleren Skalenbereich haben können, dass aber auch bei Verletzung der Annahmen eine entsprechende Energieverteilung beobachtet werden kann.

So kann es sein, dass sich das Gesetz, insbesondere da es experimentell bestätigt wurde, als richtig erweist, auch wenn sich die bisherige Herleitung bzw. Begründung als falsch herausstellt.

\subsection{Ansätze zur Turbulenzmodellierung}

Ziel der Turbulenzmodellierung ist die Verringerung der Anforderungen an die Auflösung der räumlichen und zeitlichen Diskretisierung. Dazu zählen die Verfahren der statistischen und räumlichen Mittelung der Gleichungen, so dass gemittelte Größen berechnet werden, die nur noch Strukturen bis zu einer bestimmten Längenskala oder Wellenzahl beinhalten.

Die Turbulenzmodelle lassen sich nach dem Grad der Modellierung ordnen. Durch eine statistische Mittelung (RANS) werden alle Skalen des mittleren und dissipativen Bereichs, d.h. die gesamte Energiekaskade, modelliert. Wandgrenzschichten, die bei einer statistischen Mittelung erhalten bleiben, können zusätzlich durch analytische Wandgesetze überbrückt werden. RANS-Modelle stellen somit die geringsten Anforderungen an 
die Diskretisierung, die Modelle selbst können aber sehr komplex werden.

Bei der Large Eddy Simulation (LES) werden räumliche Filteroperatoren verwendet, deren Filterweite vom Anwender bestimmt werden kann und meist an die Aufösung der räumlichen Diskretisierung gekoppelt ist. Dabei wird vorausgesetzt, dass ein Teil des mittleren Skalenbereichs, in dem das Kolmogorovsche 5/3-Gesetz gilt, aufgelöst wird. Die fehlende Dissipation im dissipativen Bereich wird dann durch LES-Turbulenzmodelle ersetzt. Da die größten energiereichen Wirbel simuliert werden sollen, sind die Anforderungen an die Auflösung der Diskretisierung für LES-Modelle höher als bei RANS-Modellen.

Beide Ansätze lassen sich auch kombinieren, indem im wandnahen Bereich RANSModelle mit Wandfunktionen und im übrigen Gebiet LES-Modelle verwendet werden (Detached Eddy Simulation, DES). Da einige RANS- und LES- Modelle die gleichen Modellterme verwenden, kann eine Umschaltung zwischen den Modellen allein durch die Bestimmung der turbulenten Viskosität erfolgen. Bevor wir uns mit dem in dieser Arbeit verwendeten LES-Modellen befassen, werden wir daher auch kurz auf die RANSGleichungen eingehen.

\subsection{Die Reynolds-gemittelten Gleichungen}

Ein wichtiges Werkzeug zur Modellbildung turbulenter Strömungen ist die statistische Mittelung. Dabei wird die Lösung $u$ des Problems als Zufallsvariable betrachtet, die von zufälligen kleinen äußeren Störungen abhängt. Um den Einfluss dieser Störungen zu eliminieren, kann ein Experiment mehrfach durchgeführt und ein Mittelwert gebildet werden.

Definition 3.1 (Ensemble-Filter) Bei $u^{(i)}$ handele es sich um Lösungen eines Problems mit kleinen zufälligen Störungen in Anfangs- und Randbedingungen sowie der Geometrie. Dann ist der Ensemble-Filter $\langle\cdot\rangle_{N}$ definiert als

$$
\langle u(x, t)\rangle_{N}:=\frac{1}{N} \sum_{i=1}^{N} u^{(i)}(x, t) .
$$

Ändern sich die Geometrie und die Randbedingungen nicht in der Zeit, kann die Lösung auch über ein großes Zeitintervall gemittelt werden.

Definition 3.2 (Zeit-Filter) Sei $u$ die Lösung eines Problems mit zeitlich konstanten Parametern, dann ist der zeitliche Mittelungsoperator $\langle\cdot\rangle_{\Delta t}$ definiert als

$$
\langle u(x, t)\rangle_{\Delta t}:=\frac{1}{\Delta t} \int_{t}^{t+\Delta t} u(x, \tau) d \tau .
$$

In einigen Anwendungen kann es eine homogene Koordinatenrichtung geben, in die alle äußeren Größen konstant sind. Das kann z.B. die spannweitige Richtung einer Tragfläche oder die Durchflussrichtung einer Rohrleitung sein. 
Definition 3.3 (Koordinaten-Filter) Sei $u$ die Lösung eines Problems mit homogener Koordinatenrichtung $\xi$, dann ist der räumliche Koordinatenfilter $\langle\cdot\rangle_{\Delta \xi}$ definiert durch

$$
\langle u(x, t)\rangle_{\Delta \xi}:=\frac{1}{\Delta \xi} \int_{x}^{x+\Delta \xi} u(\xi, t) d \xi
$$

Es können mehrere homogene Koordinatenrichtungen existieren. Bei einer Strömung zwischen zwei parallelen, unendlich ausgedehnten Platten kann in zwei Richtungen gemittelt werden, so dass die gemittelte Lösung nur noch von einer Koordinate abhängt.

Definition 3.4 Eine Lösung des Problems nennen wir statistisch stationär, falls folgende Grenzwerte - sofern die entsprechenden Mittelungsoperatoren anwendbar sind existieren:

$$
\lim _{N \rightarrow \infty}\langle u(x, t)\rangle_{N}, \quad \lim _{\Delta t \rightarrow \infty}\langle u(x, t)\rangle_{\Delta t}, \quad \lim _{\Delta \xi \rightarrow \infty}\langle u(x, t)\rangle_{\Delta \xi} .
$$

Für voll entwickelte turbulente Grenzschichtströmungen gilt dann z.B.

$$
\langle u\rangle:=\lim _{N \rightarrow \infty}\langle u(x, t)\rangle_{N}=\lim _{\Delta t \rightarrow \infty}\langle u(x, t)\rangle_{\Delta t}=\lim _{\Delta \xi \rightarrow \infty}\langle u(x, t)\rangle_{\Delta \xi} .
$$

Die statistisch gemittelte Lösung $\langle u\rangle$ hängt nicht mehr von der Zeit und den homogenen Koordinatenrichtungen ab. Wir verwenden auch folgende Bezeichung $U=\langle u\rangle$. Bei $U$ handelt es sich nicht mehr um eine Zufallsvariable, sondern um eine deterministische Größe.

Lemma 3.5 (Filtereigenschaften) Der statistische Filter-Operator $\langle\cdot\rangle$ hat folgende Eigenschaften, die wir bei der Anwendung auf unser Gleichungssystem ausnutzen werden:

$$
\text { Linearität: } \quad\langle u+\lambda v\rangle=\langle u\rangle+\lambda\langle v\rangle \quad \forall \lambda \in \mathbb{R},
$$

Kommutativität bzgl. Ortsableitungen: $\quad\left\langle\frac{\partial}{\partial x_{j}} u\right\rangle=\frac{\partial}{\partial x_{j}}\langle u\rangle$,

$$
\text { Kommutativität bzgl. Zeitableitung: } \quad\left\langle\partial_{t} u\right\rangle=\partial_{t}\langle u\rangle \text {, }
$$$$
\text { Projektionseigenschaft: } \quad\langle\langle u\rangle\rangle=\langle u\rangle \text {. }
$$

Diese Eigenschaften werden auch bei den im nächsten Abschnitt vorgestellten räumlichen Mittelungsverfahren eine wichtige Rolle spielen.

Mit dem Mittelungsoperator lässt sich die Lösung in einen mittleren Anteil und Fluktuationen zerlegen, die im Mittel verschwinden

$$
\mathbf{u}=\langle\mathbf{u}\rangle+\mathbf{u}^{\prime}, \quad\left\langle\mathbf{u}^{\prime}\right\rangle=0 .
$$

Wir werden nun den Filteroperator auf das Oberbeck-Boussinesq-Modell anwenden um Gleichungen für die gemittelten Größen $\mathbf{U}=\langle\mathbf{u}\rangle, P=\langle p\rangle$ und $\Theta=\langle\theta\rangle$ zu erhalten. Da 
wir statistisch stationäre Lösungen annehmen, fordern wir $\langle\mathbf{f}\rangle=\mathbf{f}$ und $\langle Q\rangle=Q$. Mittels der Eigenschaften aus Lemma 3.5 erhalten wir:

$$
\begin{aligned}
-\nabla \cdot(2 \nu \mathbb{D}(\mathbf{U}))+(\mathbf{U} \cdot \nabla) \mathbf{U}+\nabla \cdot\left\langle\mathbf{u}^{\prime} \otimes \mathbf{u}^{\prime}\right\rangle+\nabla P+\beta \mathbf{g} \Theta & =\mathbf{f}, \\
\nabla \cdot \mathbf{U} & =0, \\
-\nabla \cdot(\alpha \nabla \Theta)+(\mathbf{U} \cdot \nabla) \Theta+\nabla \cdot\left\langle\mathbf{u}^{\prime} \theta^{\prime}\right\rangle & =Q .
\end{aligned}
$$

Nur bei den nichtlinearen Konvektionstermen bleiben Mittelwerte von Produkten von Fluktuationen übrig, an allen anderen Stellen verschwinden die Fluktuationsterme. Es handelt sich um den Reynolds-Spannungstensor (Reynolds-stresses) $\tau$ und den residualen Wärmestrom $\mathbf{h}$

$$
\tau\left(\mathbf{u}^{\prime}\right):=\left\langle\mathbf{u}^{\prime} \otimes \mathbf{u}^{\prime}\right\rangle \quad \text { und } \quad \mathbf{h}\left(\mathbf{u}^{\prime}, \theta^{\prime}\right)=\left\langle\mathbf{u}^{\prime} \theta^{\prime}\right\rangle .
$$

Die beiden Terme $\nabla \cdot \tau$ und $\nabla \cdot \mathbf{h}$ beschreiben also den mittleren Effekt, den kleine zufällige Fluktuationen auf die mittlere Geschwindigkeit und Temperatur haben. Dieser lässt sich mittels der "Lagrangian Theory for Turbulent Mixing" [18, Abschnitt 2.2.2.1] beschreiben. Die turbulenten Fluktuationen wirken demnach auf die gemittelten Größen wie Diffusion mit speziellen Wirbelviskositäten. Als mathematisches Modell ergeben sich folgende Terme:

- Für die Impulsgleichung wird das Lineare Wirbel-Viskositäts-Modell

$$
\tau\left(\mathbf{u}^{\prime}\right)=-2 \nu_{t} \mathbb{D}(\mathbf{U})+\frac{2}{d} k \mathbb{I}
$$

verwendet. Die Normalen-Spannungen $\frac{2}{d} k \mathbb{I}$ können wegen trace $(\mathbb{D} \mathbf{u})=0$ nicht durch den Deformationstensor modelliert werden. Sie können aber wegen $\nabla \cdot \frac{2}{d} k \mathbb{I}=\nabla \frac{2}{d} k$ vom Druck aufgenommen werden, $P \rightarrow P+\frac{2}{d} k$.

- Für die Temperaturgleichung wird analog das Gradienten-Diffusions-Modell

$$
\mathbf{h}\left(\mathbf{u}^{\prime}, \theta^{\prime}\right)=-\alpha_{t} \nabla \Theta
$$

verwendet.

Eine Vielzahl unterschiedlicher Modelle ergibt sich aus den verschiedenen Möglichkeiten zur Festlegung von $\nu_{t}$ und $\alpha_{t}$. Ein wichtiger Vertreter dieser Modelle ist das $k-\omega$-Modell [72], bei dem für die turbulente kinetische Energie $k$ und die spezifische Dissipationsrate $\omega \sim \frac{\varepsilon}{k}$ zusätzliche Modellgleichungen aufgestellt werden. Daraus lassen sich dann $\nu_{t}$ und $\alpha_{t}$ berechnen.

Durch die statistische Mittelung erhält man ein deterministisches, stationäres Problem, oft auch in einem niedriger dimensionalen Raum. Diese Probleme haben gegenüber der direkten numerischen Simulation deutlich geringere Aufösungsanforderungen und sind in vielen Bereichen der aktuelle Stand der Industrie im Produktionseinsatz. Neben dem erwähnten $k$ - $\omega$-Modell gibt es eine Vielzahl weiterer Modelle, die auf einer 
oder mehreren Zusatzgleichungen für turbulente Größen basieren. Die Auswahl eines Modells für einen bestimmten Anwendungsbereich ist schwierig und häufig werden Korrekturterme eingeführt oder Tuningparameter variiert, um zunächst eine möglichst gute Übereinstimmung mit Experimenten zu erreichen. Die Qualität der Simulationen von Strömungen, für die ein Modell nicht speziell angepasst wurde, ist daher nur schwer ohne einen experimentellen Vergleich zu bewerten.

Ein weiteres Problem entsteht bei zeitabhängigen Strömungen. Ein Beispiel ist z.B. die Raumluftströmung in einem Raum mit einer Außenwand, deren Temperatur über den Verlauf des Tages variiert. Für solche Probleme wurden URANS-Verfahren (unsteady RANS) entwickelt, die auf einer zeitlichen Mittelung über ein endliches Intervall basieren und eine zeitliche Skalenseparation annehmen. Das Zeitintervall zur Mittelung muss dabei deutlich größer als die turbulenten Zeitskalen sein, aber auch deutlich kleiner als die Zeitskalen der mittleren Strömung.

\subsection{Large Eddy Simulation}

Durch immer schnellere Computer wächst auch der Wunsch, turbulente Strömungen detailierter zu simulieren. Die statistischen Mittelungsverfahren bieten dazu aber keine Möglichkeit, den Detailgrad zu variieren. Ziel ist es, alle Strukturen der Strömung, die durch die Diskretisierung abgebildet werden können, auch korrekt zu simulieren, und nur den Anteil, der nicht aufgelöst wird, zu modellieren. Dieser Ansatz wird Large Eddy Simulation (LES), also die Simulation der großen Wirbel genannt. Turbulente Strömungen lassen sich jedoch nicht ohne Anpassung des Modells mit begrenzter Auflösung berechnen.

\subsubsection{Eine Betrachtung der Ursachen für die Probleme}

Woher kommen also die Probleme bei der diskreten Simulation turbulenter Strömungen? Es ist das Zusammenspiel von Konvektion, Diffusion und der durch die Diskretisierung begrenzten Auflösung. Der Konvektionsterm ist nichtlinear (quadratisch) und sorgt unter anderem für den Energietransfer von den großen zu den kleinen Skalen. Er ist in der Regel global energieerhaltend implementiert. Der Diffusionsterm wirkt auf allen Skalen isoliert und entzieht dem System Energie. Wegen der Diskretisierung ist die Auflösung durch die Auflösungswellenzahl $k_{r}$ begrenzt. Wie die Darstellung des Dissipationsspektrums 3.1 zeigt, wird dem System ein Großteil der Energie jedoch erst im dissipativen Bereich entzogen, der in der Regel nicht mehr von einer Diskretisierung aufgelöst werden kann. Eine energieerhaltende Diskretisierung des Konvektionsterms kann die Energie also nicht auf immer kleinere Skalen transferieren. Die zugehörige Energie wird fälschlicherweise auf die größeren Skalen zurückgeworfen, wo sie langsamer dissipiert wird. Dieser Effekt wird Aliasing genannt, und tritt z.B. auch bei der Ton- oder Bildverarbeitung auf. Ein hochfrequentes Signal, das mit zu niedriger Frequenz abgetastet wird, erzeugt in der 
Ausgabe ein falsches niedrigfrequentes Signal.

In spektralen Verfahren für die Strömungssimulation werden daher sogenannte antialiasing (oder de-aliasing) Techniken verwendet. Dabei wird der Konvektionsterm mit erweitertem Spektrum zunächst exakt ausgewertet, um dann alle in der ursprünglichen Diskretisierung nicht vorhandenen Frequenzen abzuschneiden (3/2-Regel). Eine andere Möglichkeit ist, nach jeder Auswertung so viel vom hochfrequenten Anteil abzuschneiden, dass die nächste Auswertung mit dem zur Verfügung stehenden Spektrum exakt durchgeführt werden kann (2/3-Regel). Dadurch wird die fehlende Dissipation aus dem nicht aufgelösten dissipativen Bereich in den Konvektionsterm eingebaut, indem hochfrequente Anteile direkt aus der Lösung entfernt werden. Die Dissipation auf den aufgelösten Skalen wird dabei nicht verändert. Für Finite-Elemente Verfahren stehen solche Abschneideverfahren nicht direkt zur Verfügung.

\subsubsection{Die räumliche Filterung der Modellgleichungen}

Das Abschneiden von Fourier-Koeffizienten kann als räumliche Filteroperation verstanden werden. Wir führen daher einen Filteroperator $u \mapsto \bar{u}$ zur lokalen räumlichen Mittelung einer Größe $u$ ein. Mit diesem Filteroperator ist in der Regel eine Filterweite $\delta$ verbunden, die wir in der Notation jedoch weglassen werden. Die Filterweite $\delta$ wird für das gesamte Gebiet als konstant angenommen. Der Filteroperator soll wie auch die statistische Mittelung folgende Eigenschaften besitzen

$$
\begin{aligned}
\text { Linearität: } & \overline{u+\lambda v}=\bar{u}+\lambda \bar{v} \quad \forall \lambda \in \mathbb{R}, \\
\text { Kommutativität bzgl. Ortsableitungen: } & \overline{\frac{\partial}{\partial x_{j}} u}=\frac{\partial}{\partial x_{j}} \bar{u}, \\
\text { Kommutativität bzgl. Zeitableitung: } & \overline{\partial_{t} u}=\partial_{t} \bar{u} .
\end{aligned}
$$

Eine wichtige weitere Eigenschaft, die jedoch nur wenige Filter erfüllen ist die

$$
\text { Projektionseigenschaft: } \quad \overline{\bar{u}}=\bar{u} \text {. }
$$

Um seiner Funktion gerecht zu werden, fordern wir vom Filter, dass er glättend ist, für $\delta \rightarrow 0$ gegen die ungefilterte Größe konvergiert und er hochfrequente Komponenten dämpft. Für theoretische Betrachtungen wird meist der Gauß-Filter herangezogen, da er angemessen scharf im Orts- und Frequenz-Raum ist. Außerdem ist der Gauß-Filter invertierbar (wenn auch mit schlechter Kondition). Wir schreiben den Gauß-Filter als Konvolution

$$
\bar{u}(\mathbf{x}, t)=g_{\delta} * u=\int_{\mathbb{R}^{d}} g_{\delta}(\mathbf{x}-\mathbf{y}) u(\mathbf{y}, t) d y
$$

mit einer passend zur Filterweite skalierten Normalverteilung

$$
g_{\delta}(\mathbf{x})=\left(\frac{\gamma}{\delta^{2} \pi}\right)^{d / 2} \exp \left(-\frac{\gamma\|\mathbf{x}\|_{2}^{2}}{\delta^{2}}\right), \quad \gamma=6 .
$$



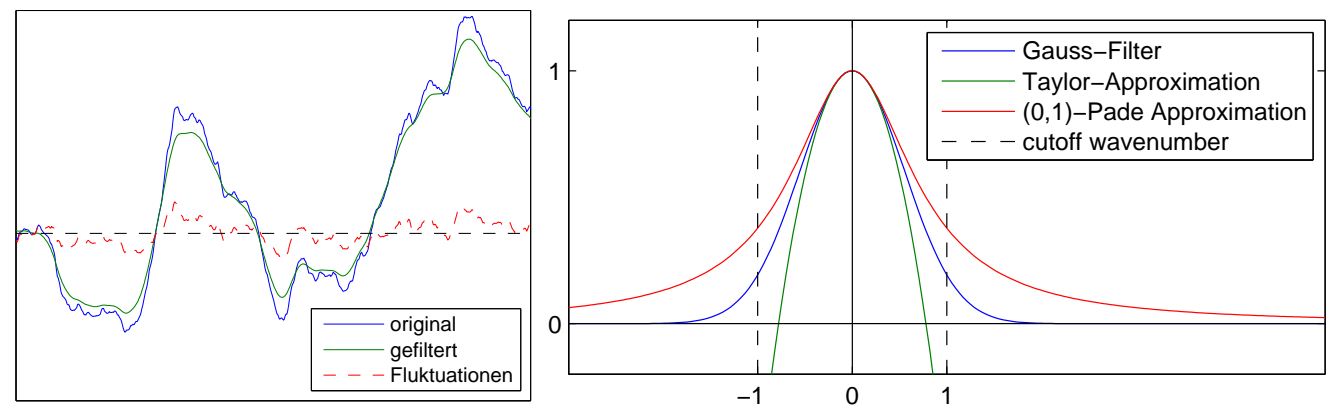

Abbildung 3.3: Gauß-Filter und Approximationen im Wellenzahlraum

Aus der Filterweite $\delta$ ergibt sich die Grenz-Wellenzahl (cutoff wavenumber) $k_{c}=\frac{\pi}{\delta}$. Da es sich bei dem Gauß-Filter nicht um eine Projektion handelt, enthält die gefilterte Größe weiterhin einen nicht vernachlässigbaren Anteil höherer Wellenzahlen. Die Auflösungs-Wellenzahl (resolution wavenumber) $k_{r}$ zur diskreten Approximation einer gefilterten Größe sollte $k_{r}=n k_{c}$ sein. Häufig wird $n=2$ verwendet, was zu $\delta=2 h$ führt. Wir wenden den Filter nun auf das Oberbeck-Boussinesq-Modell an und nutzen alle Filtereigenschaften, bis auf die Projektionseigenschaft. Die gefilterten Gleichungen lauten:

$$
\begin{aligned}
\partial_{t} \overline{\mathbf{u}}-\nabla \cdot(2 \nu \mathbb{D}(\overline{\mathbf{u}}))+(\overline{\mathbf{u}} \cdot \nabla) \overline{\mathbf{u}}+\nabla \cdot(-\overline{\mathbf{u} \otimes \mathbf{u}}+\overline{\mathbf{u}} \otimes \overline{\mathbf{u}})+\nabla \bar{p}+\beta \mathbf{g} \bar{\theta} & =\mathbf{f}, \\
\nabla \cdot \overline{\mathbf{u}} & =0, \\
\partial_{t} \bar{\theta}-\nabla \cdot(\alpha \nabla \bar{\theta})+(\overline{\mathbf{u}} \cdot \nabla) \bar{\theta}-\nabla \cdot(-\overline{\mathbf{u} \theta}+\overline{\mathbf{u}} \bar{\theta}) & =Q .
\end{aligned}
$$

Diese Gleichungen stimmen fast mit den Reynolds-gemittelten Gleichungen überein, anders als bei der statistischen Mittelung bleiben die Zeitableitungen aber erhalten. Wegen der Nicht-Kommutativität $\overline{\mathbf{u} \otimes \mathbf{u}} \neq \overline{\mathbf{u}} \otimes \overline{\mathbf{u}}$ des Filter-Operators bleiben jedoch wieder sogenannte residuale Terme:

$$
\begin{aligned}
\tau^{R} & :=\overline{\mathbf{u} \otimes \mathbf{u}}-\overline{\mathbf{u}} \otimes \overline{\mathbf{u}} & & \text { residualer Spannungstensor, } \\
k_{r} & :=\frac{1}{2} \sum_{i=1}^{d} \tau_{i i}^{R} & & \text { residualer kinetische Energie, } \\
\tau^{r} & :=\tau^{R}-\frac{2}{d} k_{r} \mathbb{I} & & \text { anisotroper residualer Spannungstensor, } \\
\mathbf{h} & :=\overline{\mathbf{u} \theta}-\overline{\mathbf{u}} \bar{\theta} & & \text { residualer Wärmestrom. }
\end{aligned}
$$

Die mittleren residualen Spannungen wirken wie Druckkräfte und werden von der Druckvariablen aufgenommen, $\bar{p} \rightarrow \bar{p}+\frac{2}{d} k_{r}$. Die residualen Terme $\tau^{r}$ und $\mathbf{h}$ müssen modelliert werden. 


\subsubsection{Das Taylor- und Rational-LES Modell}

Die beiden im Folgenden vorgestellten LES-Modelle basieren auf einer systematischen Approximation des Gauß-Filters im Wellenzahl-Raum [8, 36]. Dazu zerlegen wir

$$
\overline{\mathbf{u} \otimes \mathbf{u}}-\overline{\mathbf{u}} \otimes \overline{\mathbf{u}}=\overline{\overline{\mathbf{u}} \otimes \overline{\mathbf{u}}}-\overline{\mathbf{u}} \otimes \overline{\mathbf{u}}+\overline{\overline{\mathbf{u}} \otimes \mathbf{u}^{\prime}}+\overline{\mathbf{u}^{\prime} \otimes \overline{\mathbf{u}}}+\overline{\mathbf{u}^{\prime} \otimes \mathbf{u}^{\prime}} .
$$

Durch Anwendung der Fourier-Transformation, Taylor-Entwicklung des Gauß-Filters bezüglich der Filterweite $\delta$ und anschließende Rücktransformation mittels der inversen Fourier-Transformation erhalten wir:

$$
\begin{aligned}
& \overline{\overline{\mathbf{u}} \otimes \overline{\mathbf{u}}}-\overline{\mathbf{u}} \otimes \overline{\mathbf{u}}=\frac{\delta^{2}}{4 \gamma} \Delta(\overline{\mathbf{u}} \otimes \overline{\mathbf{u}})+\mathcal{O}_{\text {formal }}\left(\delta^{4}\right) \quad \text { Leonard-Tensor }, \\
& \overline{\overline{\mathbf{u}} \otimes \mathbf{u}^{\prime}}=-\frac{\delta^{2}}{4 \gamma}(\overline{\mathbf{u}} \otimes \Delta \overline{\mathbf{u}})+\mathcal{O}_{\text {formal }}\left(\delta^{4}\right) \\
& \overline{\mathbf{u}^{\prime} \otimes \overline{\mathbf{u}}}=-\frac{\delta^{2}}{4 \gamma}(\Delta \overline{\mathbf{u}} \otimes \overline{\mathbf{u}})+\mathcal{O}_{\text {formal }}\left(\delta^{4}\right) \\
& \overline{\mathbf{u}^{\prime} \otimes \mathbf{u}^{\prime}}=\frac{\delta^{4}}{16 \gamma^{2}}(\Delta \overline{\mathbf{u}} \otimes \Delta \overline{\mathbf{u}})+\mathcal{O}_{\text {formal }}\left(\delta^{6}\right) \quad \text { Sub-Filter Spannungstensor. }
\end{aligned}
$$

Der residuale Wärmestrom $\mathbf{h}$ kann analog approximiert werden. Zusammenfassen aller Terme bis zur zweiten Ordnung liefert das Taylor-LES-Turbulenzmodell (auch GradientLES-Turbulenzmodell):

$$
\tau^{r} \approx \frac{\delta^{2}}{2 \gamma}\left(\nabla \overline{\mathbf{u}}: \nabla \overline{\mathbf{u}}^{\top}\right), \quad \mathbf{h} \approx \frac{\delta^{2}}{2 \gamma}\left(\nabla \bar{\theta}: \nabla \overline{\mathbf{u}}^{\top}\right) .
$$

Diese polynomielle Approximation des Gauß-Filters zeigt bei a-priori Tests gute Konsistenzeigenschaften, reproduziert aber die Dämpfungseigenschaft für große Wellenzahlen nicht. Da die Modellterme nicht strikt dissipativ sind, ergeben sich Stabilitätsprobleme, die dazu führen können, dass die Energie der Lösung exponentiell zunimmt (blow up) [36].

Bessere Eigenschaften hat eine rationale Approximation des Gauß-Filters im WellenzahlRaum. Diese führt auf die approximierte Konvolution

$$
\overline{\mathbf{w}} \approx \mathcal{R}(\mathbf{w}):=\left(-\frac{\delta}{4 \gamma} \Delta+\mathbb{I}\right)^{-1} \mathbf{w} .
$$

Sie hat für kleine Wellenzahlen die gleiche Approximationseigenschaft wie die polynomielle Approximation, reproduziert jedoch für große Wellenzahlen die Dämpfungseigenschaft des Gauß-Filters (siehe Abbildung 3.3). Analog zum Taylor-LES Modell erhalten wir das Rational-LES-Modell:

$$
\tau^{r} \approx \mathcal{R}\left(\frac{\delta^{2}}{2 \gamma} \nabla \overline{\mathbf{u}}: \nabla \overline{\mathbf{u}}^{\top}\right), \quad \mathbf{h} \approx \mathcal{R}\left(\frac{\delta^{2}}{2 \gamma} \nabla \bar{\theta}: \nabla \overline{\mathbf{u}}^{\top}\right)
$$


Auch mit dem Rational-LES-Modell ist weiterhin ein blow-up der Energie möglich, numerische Untersuchungen zeigen jedoch deutlich verbesserte Stabilitätseigenschaften gegenüber dem Gradient-LES Modell [36, 21].

Bei $\mathcal{R}$ handelt es sich um einen sogenannten differentiellen Filter, der selbst eine Approximation an den Gauß-Filter ist. Diese Beobachtung legt die Idee nahe, statt $\mathcal{R}$ wieder den Gauß-Filter einzusetzen. Dieses Modell nennen wird Rational-LES mittels Konvolution

$$
\tau^{r} \approx g_{\delta} *\left(\frac{\delta^{2}}{2 \gamma} \nabla \overline{\mathbf{u}}: \nabla \overline{\mathbf{u}}^{T}\right), \quad \mathbf{h} \approx g_{\delta} *\left(\frac{\delta^{2}}{2 \gamma} \nabla \bar{\theta}: \nabla \overline{\mathbf{u}}^{T}\right) .
$$

Die in diesem Abschnitt vorgestellten Modelle basieren nur auf den einfachsten Ansätzen zur Approximation des Filters mit zweiter Ordnung und lassen sich auf höhere Ordnungen erweitern. Ein Vorteil dieser Verfahren ist, dass sie sich systematisch herleiten lassen und keine unbekannten Tuning-Parameter enthalten. Andererseits zeigt das unterschiedliche Verhalten des Taylor- und Rational-LES Ansatzes, dass eine Modellbildung nicht allein auf Konsistenzeigenschaften beruhen kann.

\subsubsection{Das Lilly-Eidson Modell}

Statt auf einer systematischen Approximation der residualen Terme kann ein Turbulenzmodell auch auf dem Ziel basieren, das Kolmogorov-5/3-Gesetz zur Energieverteilung zu reproduzieren. Beim Lilly-Eidson Modell wird die fehlende Dissipation auf den nicht aufgelösten Skalen durch das Hinzufügen von globaler Diffusion auf allen aufgelösten Skalen ausgeglichen. Es handelt sich wie auch bei vielen RANS-Modellen um ein Wirbelviskositätsmodell mit

$$
\nu_{T}=C_{E} \delta^{2} \max \left(0 ; \overline{\mathcal{D}}^{2}+\frac{\beta}{\operatorname{Pr}_{t}} \mathbf{g} \cdot \nabla \bar{\theta}\right)^{1 / 2} \quad \text { und } \quad \alpha_{T}=\operatorname{Pr}_{T}^{-1} \nu_{T},
$$

wobei

$$
\overline{\mathcal{D}}:=\sqrt{\mathbb{D}(\overline{\mathbf{u}}): \mathbb{D}(\overline{\mathbf{u}})}=\|\mathbb{D}(\overline{\mathbf{u}})\|_{F} .
$$

Das Modell vereinfacht sich für isotherme Strömungen mit $\nabla \bar{\theta}=\mathbf{0}$ zum SmagorinskyModell $\nu_{T}=C_{E} \delta^{2} \overline{\mathcal{D}}$. Eine Linearisierung des Wurzelausdruckes in (3.25) gemäß $\left(\overline{\mathcal{D}}^{2}+x\right)^{1 / 2} \approx \overline{\mathcal{D}}+\frac{1}{2} \frac{x}{\overline{\mathcal{D}}}$ führt auf

$$
\nu_{T}=\max \left(-\nu ; C_{E} \delta^{2} \overline{\mathcal{D}}^{-1}\left(\overline{\mathcal{D}}^{2}+\frac{\beta}{2 \operatorname{Pr}_{t}} \mathbf{g} \cdot \nabla \bar{\theta}\right)\right), \quad \alpha_{T}=\operatorname{Pr}_{T}^{-1} \nu_{T}
$$

Als Begründung für diese Definitionen soll folgende Betrachtung dienen. Ausgehend von Arbeiten von Lilly [46, 47] und Eidson [19] ist der gesamte Energietransfer von den gefilterten zu den residualen Skalen $P_{r}^{\theta}$ durch

$$
P_{r}^{\theta} \equiv-\tau^{r}: \mathbb{D}(\overline{\mathbf{u}})-\beta \mathbf{g} \cdot \mathbf{h}
$$


gegeben. Das Turbulenzmodell ersetzt die Dissipation $\varepsilon$ auf den nicht aufgelösten Skalen. Da die Energie fast ausschließlich im nicht aufgelösten dissipativen Bereich dissipiert wird, soll $\varepsilon \approx P_{r}^{\theta}$ gelten. Mit der Definition (3.26) ist

$$
\begin{aligned}
\varepsilon=2 \nu_{t} \mathcal{D}^{2} & =2 C_{E} \delta^{2} \overline{\mathcal{D}}^{-1}\left(\overline{\mathcal{D}}^{2}+\frac{\beta}{2 \operatorname{Pr}_{t}} \mathbf{g} \cdot \nabla \bar{\theta}\right) \overline{\mathcal{D}}^{2} \\
& =2\left[C_{E} \delta^{2} \overline{\mathcal{D}}\right] \overline{\mathcal{D}}^{2}+\beta \frac{C_{E} \delta^{2} \overline{\mathcal{D}}}{\operatorname{Pr}_{t}} \mathbf{g} \cdot \nabla \bar{\theta}
\end{aligned}
$$

Dabei handelt es sich unter Verwendung der Wirbel-Viskositäts- und der GradientenDiffusion-Annahme um eine konsistente Approximation an $P_{r}^{\theta}$. Wegen des Zusammenhangs zwischen (3.26) und (3.25) gilt dies entsprechend auch für (3.25).

Die noch unbestimmten Parameter werden von Eidson in [19] mit $C_{E}=0.0441$ und $\operatorname{Pr}_{T}=0.4$ angegeben. Zur Festlegung dieser Parameter wird auch das KolmogorovGesetz verwendet, um die Dissipation $\varepsilon$ zu bestimmen. Vergleiche mit dynamisch bestimmten Parametern zeigen eine gute Übereinstimmung mit den konstanten Werten [58].

\subsubsection{LES mittels VMS-Turbulenzmodellen}

Beim Lilly-Eidson Modell wurde die spektrale Verteilung der Dissipation nicht berücksichtigt. Dadurch findet ein nicht unerheblicher Teil der Dissipation auch auf den großen Skalen statt, was dazu führt, dass das Modell häufig zu dissipativ ist. Dies ist insbesondere dann der Fall, wenn es sich um nur wenig turbulente oder sogar laminare Strömungen handelt. Solche Strömungen sollten nur wenig vom Turbulenzmodell beinflusst werden. Es liegt also nahe, wie bei den anti-aliasing Techniken spektraler Verfahren, nur den hochfrequenten Anteil der diskreten Lösung zu verändern. Wir werden die Dissipation auf den kleinen aufgelösten Skalen erhöhen, um die fehlende Dissipation der nicht aufgelösten Skalen auszugleichen. So erzeugen wir einen künstlichen dissipativen Bereich, der innerhalb der Aufösung der Diskretisierung liegt. Falls nur wenig Energie auf diese kleinen aufgelösten Skalen verteilt ist, z.B. bei laminaren Strömungen, so hat auch das Turbulenzmodell nur einen kleinen Einfluss auf die Lösung.

Für die verwendeten Finite-Elemente Räume müssen wir daher eine Möglichkeit schaffen, die aufgelösten Skalen in zwei Bereiche zu zerlegen. Da wir die Fluktuationen des Deformationstensors der Geschwindigkeit und des Gradienten der Temperatur erfassen wollen, benötigen wir Grobräume $\mathbf{L}_{H}, \mathbf{M}_{H}$ mit entsprechender Dimension

$$
\begin{gathered}
\{0\} \subseteq \mathbf{L}_{H} \subseteq \mathbf{L}:=\left\{\left(l_{i j}\right) \in\left[L^{2}(\Omega)\right]^{3 \times 3} \mid l_{i j}=l_{j i}\right\}, \\
\{0\} \subseteq \mathbf{M}_{H} \subseteq \mathbf{M}:=\left[L^{2}(\Omega)\right]^{3} .
\end{gathered}
$$

Für die Zerlegung werden wir $L^{2}$-orthogonale Projektionen verwenden

$$
\Pi_{H}^{u}: \mathbf{L} \rightarrow \mathbf{L}_{H} \subset \mathbf{L}, \quad \Pi_{H}^{\theta}: \mathbf{M} \rightarrow \mathbf{M}_{H} \subset \mathbf{M} .
$$


Diese sind wie üblich durch $\Pi_{H}^{u}(\mathbb{D} \mathbf{u}):=\mathbf{G}$ und $\Pi_{H}^{\theta}(\nabla \theta):=\mathbf{F}$ mittels folgender Variationsgleichungen definiert

$$
\left(\mathbf{G}-\mathbb{D} \mathbf{u}, L_{H}\right)=0, \quad \forall L_{H} \in \mathbf{L}_{H} \quad \text { und } \quad\left(\mathbf{F}-\nabla \theta, M_{H}\right)=0, \quad \forall M_{H} \in \mathbf{M}_{H} .
$$

Definition 3.6 Mittels der oben definierten Projektionen $\Pi_{H}^{u}, \Pi_{H}^{\theta}$ auf die Grobräume $\mathbf{L}_{H}, \mathbf{M}_{H}$ seien die Fluktuationsoperatoren

$$
\kappa_{u}:=\mathrm{I}_{L}-\Pi_{H}^{u}: \mathbf{L} \rightarrow \mathbf{L}_{/ \mathbf{L}_{H}}, \quad \kappa_{\theta}:=\mathrm{I}_{M}-\Pi_{H}^{\theta}: \mathbf{M} \rightarrow \mathbf{M}_{/ \mathbf{M}_{H}}
$$

definiert.

Zur Turbulenzmodellierung führen wir folgende SGS-Modellterme (subgrid-scale)

$$
\sum_{K \in \mathcal{T}_{h}} \nu_{K}^{T}\left(\kappa_{u}\left(\mathbb{D} \mathbf{u}_{h}\right), \kappa_{u}\left(\mathbb{D} \mathbf{v}_{h}\right)\right)_{K}, \quad \sum_{K \in \mathcal{T}_{h}} \alpha_{K}^{T}\left(\kappa_{\theta}\left(\nabla \theta_{h}\right), \kappa_{\theta}\left(\nabla \psi_{h}\right)\right)_{K}
$$

mit zellweise konstanten turbulenten Viskositätsparametern $\nu_{K}^{T}, \alpha_{K}^{T}$ ein. Da die Fluktuationsoperatoren $\kappa_{u / \theta}$ nicht mit den Gradienten kommutieren, entsprechen diese Modellterme nicht Diffusionstermen. Das lässt sich jedoch unter einer Zusatzannahme an $\mathbf{L}_{H}, \mathbf{M}_{H}$ durch Einführung einer speziellen elliptischen Projektion reproduzieren. Für die Grobräume gelte

$$
\mathbf{L}_{H} \subseteq \mathbb{D} \mathbf{V}_{h}, \quad \mathbf{M}_{H} \subseteq \nabla \Theta_{h},
$$

dann gibt es elliptische Projektionen $\Pi^{u}, \Pi^{\theta}$, die durch

$$
\Pi_{H}^{u}\left(\mathbb{D} \mathbf{u}_{h}\right)=\mathbb{D}\left(\Pi^{u} \mathbf{u}_{h}\right), \quad \Pi_{H}^{\theta}\left(\nabla \theta_{h}\right)=\nabla\left(\Pi^{\theta} \theta_{h}\right) .
$$

definiert sind. Damit lassen sich die Fluktuationsterme umstellen:

$$
\begin{aligned}
\kappa_{u}\left(\mathbb{D} \mathbf{u}_{h}\right) & =\mathrm{I}_{L}\left(\mathbb{D} \mathbf{u}_{h}\right)-\Pi_{H}^{u}\left(\mathbb{D} \mathbf{u}_{h}\right)=\mathbb{D} \mathbf{u}_{h}-\mathbb{D}\left(\Pi^{u} \mathbf{u}_{h}\right)=\mathbb{D}\left(\left(\mathrm{I}-\Pi^{u}\right)\left(\mathbf{u}_{h}\right)\right), \\
\kappa_{\theta}\left(\nabla \theta_{h}\right) & =\mathrm{I}_{L}\left(\nabla \theta_{h}\right)-\Pi_{H}^{\theta}\left(\nabla \theta_{h}\right)=\nabla \theta_{h}-\nabla\left(\Pi^{\theta} \theta_{h}\right)=\nabla\left(\left(\mathrm{I}-\Pi^{\theta}\right)\left(\theta_{h}\right)\right) .
\end{aligned}
$$

Unter der Annahme (3.32) können die SGS-Modellterme also tatsächlich als Diffusionsterme aufgefasst werden. Doch auch ohne die Inklusion lässt sich wegen der lokalen $L^{2}(K)$-Orthogonalität der Projektion jedoch feststellen, dass

$$
\begin{aligned}
& \sum_{K \in \mathcal{T}_{h}} \nu_{K}^{T}\left\|\kappa_{u}\left(\mathbb{D} \mathbf{u}_{h}\right)\right\|_{L^{2}(K)}^{2}=\sum_{K \in \mathcal{T}_{h}} \nu_{K}^{T}\left(\left\|\mathbb{D} \mathbf{u}_{h}\right\|_{L^{2}(K)}^{2}-\left\|\Pi_{H}^{u}\left(\mathbb{D} \mathbf{u}_{h}\right)\right\|_{L^{2}(K)}^{2}\right) \\
&=\sum_{K \in \mathcal{T}_{h}} \nu_{K}^{T}\left(1-\frac{\left\|\Pi_{H}^{u}\left(\mathbb{D} \mathbf{u}_{h}\right)\right\|_{L^{2}(K)}^{2}}{\left\|\mathbb{D} \mathbf{u}_{h}\right\|_{L^{2}(K)}^{2}}\right)\left\|\mathbb{D} \mathbf{u}_{h}\right\|_{L^{2}(K)}^{2}=\sum_{K \in \mathcal{T}_{h}} \nu_{K}^{\mathrm{VMS}}\left\|\mathbb{D} \mathbf{u}_{h}\right\|_{L^{2}(K)}^{2}
\end{aligned}
$$

mit $\nu_{K}^{\mathrm{VMS}} \geq 0$. Der Modellterm hat auf die Gesamtenergiebilanz also den gleichen Einfluss wie ein Diffusionsterm mit reduzierter turbulenter Viskosität $\nu_{K}^{\mathrm{VMS}}$. Diese Reduktion ist jedoch von der spektralen Verteilung der Energie abhängig. Für glatte Lösungen, 
bei denen keine Energie in den kleinsten aufgelösten Skalen steckt, geht $\nu_{K}^{\mathrm{VMS}} / \nu_{K}^{T} \rightarrow 0$. Dies ist ein entscheidender Vorteil z.B. gegenüber dem Smagorinsky-Modell, da sich das Turbulenzmodell für laminare Strömungen automatisch ausschaltet. Bei turbulenten Strömungen wird das Turbulenzmodell durch die in den kleinen aufgelösten Skalen steckende Energie aktiviert. Daher werden wir die SGS-Modellterme (3.31) auch ohne die Inklusion (3.32) verwenden, wodurch wir größere Freiheit bei der Wahl der Grobräume erhalten.

Für die turbulenten Viskositätsparameter $\nu_{K}^{T}, \alpha_{K}^{T}$ werden wir wieder die Formeln (3.25) verwenden. Die Konstante $C_{E}$ muss jedoch neu bestimmt werden und hängt von der Diskretisierung und den verwendeten Grobräumen ab. Für die hier verwendeten Modellterme wurde in [62] die Analysis von Lilly [47] erweitert. Wir zitieren das Resultat:

$$
C_{E}=\left(\frac{4}{3 K_{0}}\right)^{3 / 2} \frac{1}{\pi^{2}}\left(1-\left(\frac{k_{c}}{k_{f}}\right)^{4 / 3}\right)^{-3 / 2}
$$

Bei $K_{0}$ handelt es sich um die Kolmogorov-Konstante, deren Wert in [68] mit Daten aus einer Vielzahl von Experimenten mit $K_{0} \approx 1.53$ bestimmt wurde. Bei $k_{c}$ und $k_{f}$ handelt es sich um Auflösungswellenzahlen für die Diskretisierung und die Grobräume. Der Quotient beschreibt also den Anteil der aufgelösten Skalen, die auch vom Grobraum aufgelöst werden. Für die numerischen Simulationen werden die in [62] bestimmten Werte verwendet.

Bemerkung 3.2 Die SGS-Modellterme (3.31) wurden bereits unter der Bezeichnung Gradienten-basierte Stabilisierung durch lokale Projektion als Stabilisierungsterme für das Oseen-Problem (eine Linearisierung der Navier-Stokes-Gleichungen) und für skalare Konvektions-Diffusions-Probleme verwendet [55, 51]. Da es sich dort nicht um Turbulenzmodelle handelt, wird zur Bestimmung der Parameter $\nu_{K}^{T}$ und $\alpha_{K}^{T}$ eine a-priori Fehlerabschätzung verwendet, die durch die Wahl der Parameter optimiert wird.

Das vollständige Modell für nicht-isotherme turbulente Strömungen mit VMSTurbulenzmodell lautet wie folgt.

Oberbeck-Boussinesq-Modell mit VMS-Turbulenzmodell:

$$
\begin{aligned}
\left(\partial_{t} \mathbf{u}_{h}, \mathbf{v}_{h}\right)+\left(2 \nu \mathbb{D} \mathbf{u}_{h}, \mathbb{D} \mathbf{v}_{h}\right)+b_{s}\left(\mathbf{u}_{h}, \mathbf{u}_{h}, \mathbf{v}_{h}\right) & \\
-\left(p_{h}, \nabla \cdot \mathbf{v}_{h}\right)+\left(\beta \theta_{h} \mathbf{g}, \mathbf{v}_{h}\right) & \\
+\sum_{K \in \mathcal{T}_{h}}\left[\nu_{K}^{T}\left(\kappa_{u}\left(\mathbb{D} \mathbf{u}_{h}\right), \kappa_{u}\left(\mathbb{D} \mathbf{v}_{h}\right)\right)+\gamma_{K}\left(\nabla \cdot \mathbf{u}_{h}, \nabla \cdot \mathbf{v}_{h}\right)\right] & =\left(\mathbf{f}, \mathbf{v}_{h}\right), \\
\left.\left(\partial_{t} \theta_{h}, \psi_{h}\right)+\left(\alpha \nabla \theta_{h}, \nabla \psi_{h}\right)+c_{s}, q_{h}\right) & =0, \\
+\sum_{K \in \mathcal{T}_{h}} \alpha_{K}^{T}\left(\kappa_{\theta}\left(\nabla \theta_{h}\right), \kappa_{\theta}\left(\nabla \psi_{h}\right)\right) & =\left(Q, \psi_{h}\right) .
\end{aligned}
$$




\subsection{Die Implementierung der SGS-Modellterme}

Die Multiskalen-Diffusionsoperatoren lassen sich auf verschiedene Weisen implementieren. Wir betrachten dazu exemplarisch den Fall für die Temperatur. Für die Geschwindigkeit kann völlig analog vorgegangen werden. Ziel ist also die Implementierung von

$$
\sum_{K \in \mathcal{T}_{h}} \alpha_{K}^{T}\left(\kappa_{\theta}\left(\nabla \theta_{h}\right), \kappa_{\theta}\left(\nabla \psi_{h}\right)\right)
$$

Wir nutzen dazu die Definition (3.30) von $\kappa_{\theta}$ und die Definition (3.29) von $\Pi_{h}^{\theta}\left(\nabla \theta_{h}\right)$. Damit ersetzen wir in (3.35) zunächst

$$
\sum_{K \in \mathcal{T}_{h}} \alpha_{K}^{T}\left(\kappa_{\theta}\left(\nabla \theta_{h}\right), \kappa_{\theta}\left(\nabla \psi_{h}\right)\right)_{K}=\sum_{K \in \mathcal{T}_{h}} \alpha_{K}^{T}\left(\nabla \theta_{h}, \nabla \psi_{h}\right)_{K}-\sqrt{\alpha_{K}^{T}}\left(\mathbf{F}, \nabla \psi_{h}\right)_{K}
$$

und fügen die Variationsgleichung der zellweise skalierten $L^{2}$-Projektion

$$
\sum_{K \in \mathcal{T}_{h}}\left(\mathbf{F}-\sqrt{\alpha_{K}^{T}} \nabla \theta_{h}, M_{H}\right)_{K}=0, \quad \forall M_{H} \in \mathbf{M}_{H}
$$

zum System (3.33) bis (3.35) hinzu. Die turbulente Viskosität wurde dabei auf beide Gleichungen verteilt, um den folgenden Schritt zu vereinfachen. Für die Anwendung des Ritz-Galerkin-Verfahrens, wird eine Basis von $\mathbf{M}_{H}$ mit der Dimension $n_{\theta, H}:=\operatorname{dim}\left(\mathbf{M}_{H}\right)$ benötigt:

$$
\mathbf{M}_{H}=\operatorname{span}\left\{\xi_{k} \mid k=1, \ldots, n_{\theta, H}\right\} .
$$

Außerdem verwenden wir noch die Basis $\psi_{l}$ von $\Theta_{h}$ zur Definition der Matrizen

$$
\begin{gathered}
M_{\theta, H}=\left[\left(\xi_{l}, \xi_{k}\right)_{\Omega}\right]_{k, l} \in \mathbb{R}^{n_{\theta, H} \times n_{\theta, H},} \\
D_{\theta}=\left[\sum_{K \in \mathcal{T}_{h}} \alpha_{K}^{T}\left(\nabla \psi_{j}, \nabla \psi_{i}\right)_{K}\right]_{i, j} \in \mathbb{R}^{n_{\theta} \times n_{\theta},} \\
T_{\theta}=\left[\sum_{K \in \mathcal{T}_{h}} \sqrt{\alpha_{K}^{T}}\left(\nabla \psi_{j}, \xi_{k}\right)_{K}\right]_{k, j} \in \mathbb{R}^{n_{\theta, H} \times n_{\theta}} .
\end{gathered}
$$

Die durch diese Matrizen bezüglich der gewählten Basen dargestellten Operatoren bilden die Räume folgendermaßen ab:

$$
M_{\theta, H}: \mathbf{M}_{H} \rightarrow \mathbf{M}_{H}^{*}, \quad D_{\theta}: Q_{h} \rightarrow Q_{h}^{*}, \quad T_{\theta}: Q_{h} \rightarrow \mathbf{M}_{H}^{*}, \quad T_{\theta}^{\top}: \mathbf{M}_{H} \rightarrow Q_{h}^{*} .
$$

Das System (3.33) bis (3.35) und (3.36) lautet dann in Operator- bzw. Block-MatrixForm:

$$
\left(\begin{array}{cccc}
M_{u} & 0 & 0 & 0 \\
0 & M_{\theta} & 0 & 0 \\
0 & 0 & 0 & 0 \\
0 & 0 & 0 & 0
\end{array}\right)\left(\begin{array}{c}
\mathbf{u}_{h}^{\prime} \\
\theta_{h}^{\prime} \\
p_{h}^{\prime} \\
\mathbf{F}^{\prime}
\end{array}\right)=\left(\begin{array}{c}
\hat{\mathbf{f}} \\
\widehat{Q} \\
0 \\
0
\end{array}\right)-\left(\begin{array}{cccc}
A_{u}\left(\mathbf{u}_{h}\right) & C & B^{\top} & 0 \\
0 & A_{\theta}\left(\mathbf{u}_{h}\right)+D_{\theta} & 0 & T_{\theta}^{\top} \\
B & 0 & 0 & 0 \\
0 & -T_{\theta} & 0 & M_{\theta, H}
\end{array}\right)\left(\begin{array}{c}
\mathbf{u}_{h} \\
\theta_{h} \\
p_{h} \\
\mathbf{F}
\end{array}\right) .
$$


Da $\mathbf{M}_{H}$ ein Ansatzraum für Gradienten der Temperatur ist, hat dieses System jedoch eine wesentlich größere Dimension als das ursprüngliche System (2.21). Wegen der Invertierbarkeit der Massematrix $M_{\theta, H}$ lässt sich $\mathbf{F}$ jedoch eliminieren, so dass wir das System

$$
\left(\begin{array}{ccc}
M_{u} & 0 & 0 \\
0 & M_{\theta} & 0 \\
0 & 0 & 0
\end{array}\right)\left(\begin{array}{c}
\mathbf{u}_{h}^{\prime} \\
\theta_{h}^{\prime} \\
p_{h}^{\prime}
\end{array}\right)=\left(\begin{array}{c}
\hat{\mathbf{f}} \\
\widehat{Q} \\
0
\end{array}\right)-\left(\begin{array}{ccc}
A_{u}\left(\mathbf{u}_{h}\right) & C & B^{\top} \\
0 & A_{\theta}\left(\mathbf{u}_{h}\right)+D_{\theta}-T_{\theta}^{\top} M_{\theta, H}^{-1} T_{\theta} & 0 \\
B & 0 & 0
\end{array}\right)\left(\begin{array}{c}
\mathbf{u}_{h} \\
\theta_{h} \\
p_{h}
\end{array}\right)
$$

mit der gleichen Dimension wie (2.21) erhalten. Im Produkt $T_{\theta}^{\top} M_{\theta, H}^{-1} T_{\theta}$ wird auch die turbulente Viskosität wieder zum richtigen Wert quadriert. $D_{\theta}$ kann durch Abändern von $\alpha \rightarrow \alpha+\alpha_{K}^{T}$ effizient direkt in $A_{\theta}$ berechnet werden.

Die einzige Chance $M_{\theta, H}^{-1}$ effizient zu berechnen, besteht in der Wahl eines unstetigen Ansatzraumes für $\mathbf{M}_{H}$. Dann handelt es sich bei $M_{\theta, H}$ und $T_{\theta}$ um Blockmatrizen und das Produkt $T_{\theta}^{\top} M_{\theta, H}^{-1} T_{\theta}$ kann zellweise berechnet werden. So kann das System (3.37) explizit in Matrixform aufgestellt werden. Die Vorgehensweise für den anderen Modellterm ist völlig analog.

\subsection{Zusammenfassung}

In diesem Kapitel wurde die Notwendigkeit eines Turbulenzmodells für turbulente Strömungen begründet und unterschiedliche Ansätze zur Turbulenzmodellierung vorgestellt. Die Konstruktion und Details zur Implementierung eines neuen VMSTurbulenzmodells für das Oberbeck-Boussinesq-Modell wurden ausführlich dargestellt. Im nächsten Kapitel folgt eine a-priori Fehleranalysis für dieses Modell. Die Wahl der Grobräume $\mathbf{L}_{H}, \mathbf{M}_{H}$ und der Filterweite $\delta$ wird erneut im Anschluss an die Fehlerabschätzung diskutiert und später in numerischen Experimenten untersucht. 


\section{Kapitel 4}

\section{Analysis zum semi-diskreten Problem}

Das Ziel dieses Kapitels ist eine a-priori Fehlerabschätzung für das räumlich diskretisierte Oberbeck-Boussinesq-Modell mit VMS-Turbulenzmodell. Für das isotherme Problem ist eine ähnliche Analysis für global konstante Turbulenzparameter in einer Arbeit von John [38] zu finden. Ebenfalls für das isotherme Problem wurde diese Fehlerabschätzung in [63] auf nichtlinear von der Lösung abhängige Turbulenzparameter erweitert. Die folgenden Resultate wurden zur Veröffentlichung in Mathematical Models and Methods in Applied Sciences eingereicht und im Mai 2011 akzeptiert [53] und sind in einer Kurzfassung in den Proceedings zur BAIL 2010 [54] erschienen.

Wie in Abschnitt 2.1 können wir auch bei der diskreten Variationsformulierung (3.33) bis (3.35) zu einer reduzierten Variationsformulierung für $\mathbf{u}_{h}, \theta_{h}:[0, T] \rightarrow \mathbf{Z}_{h} \times \Theta_{h}$ übergehen:

$$
\begin{aligned}
\left(\partial_{t} \mathbf{u}_{h}, \mathbf{z}_{h}\right)+\left(2 \nu \mathbb{D} \mathbf{u}_{h}, \mathbb{D} \mathbf{z}_{h}\right)+\left(\left(\mathbf{u}_{h} \cdot \nabla\right) \mathbf{u}_{h}, \mathbf{z}_{h}\right)+\left(\beta \theta_{h} \mathbf{g}, \mathbf{z}_{h}\right) & \\
+\sum_{K \in \mathcal{T}_{h}}\left[\nu_{K}^{T}\left(\kappa_{u}\left(\mathbb{D} \mathbf{u}_{h}\right), \kappa_{u}\left(\mathbb{D} \mathbf{z}_{h}\right)\right)+\gamma_{K}\left(\nabla \cdot \mathbf{u}_{h}, \nabla \cdot \mathbf{z}_{h}\right)\right] & =\left(\mathbf{f}, \mathbf{v}_{h}\right), \\
\left(\partial_{t} \theta_{h}, \psi_{h}\right)+\left(\alpha \nabla \theta_{h}, \nabla \psi_{h}\right)+\left(\left(\mathbf{u}_{h} \cdot \nabla\right) \theta_{h}, \psi_{h}\right) & \\
+\sum_{K \in \mathcal{T}_{h}} \alpha_{K}^{T}\left(\kappa_{\theta}\left(\nabla \theta_{h}\right), \kappa_{\theta}\left(\nabla \psi_{h}\right)\right) & =\left(Q, \psi_{h}\right)
\end{aligned}
$$

für alle $t \in[0, T]$ und beliebige Testfunktionen $\mathbf{z}_{h} \in \mathbf{Z}_{h}, \psi_{h} \in \Theta_{h}$. Wir werden zunächst aus diesen Gleichungen eine Fehlerabschätzung nur für das Geschwindigkeitsfeld $\mathbf{u}_{h}$ und die Temperatur $\theta_{h}$ herleiten. 


\subsection{Vorbereitungen}

Wir beginnen in diesem Abschnitt mit einigen elementaren Ungleichungen, die im Verlauf dieses Kapitels wiederholt verwendet werden.

Lemma 4.1 (Cauchy-Schwarzsche Ungleichung) Es sei $V$ ein Hilbert-Raum mit dem Skalarprodukt $(\cdot, \cdot)_{V}$, dann gilt

$$
(u, v)_{V} \leq\|u\|_{V}\|v\|_{V}
$$

für alle $u, v \in V$. Ein Abschätzung der dualen Paarung ergibt

$$
\langle w, v\rangle_{V^{*}, V}=(\mathcal{R}(w), v)_{V} \leq\|\mathcal{R}(w)\|_{V}\|v\|_{V}=\|w\|_{V^{*}}\|v\|_{V}
$$

für alle $w \in V^{*}, v \in V$. Dabei ist $\mathcal{R}: V^{*} \rightarrow V$ der isometrische Rieszsche Darstellungsoperator.

Lemma 4.2 (Youngsche Ungleichung) Es seien $p, q \in(1, \infty)$ mit $\frac{1}{p}+\frac{1}{q}=1$ und $\epsilon>0$ beliebig, dann gilt

$$
a b \leq \frac{\epsilon}{p} a^{p}+\frac{\epsilon^{-q / p}}{q} b^{q}
$$

für alle $a, b \geq 0$.

Die Youngsche Ungleichung werden wir zur Zerlegung von Produkten aus Diskretisierungs- und Interpolationsfehlern einsetzen. Eine geeignete Wahl von $\epsilon$ wird dabei eine wichtige Rolle spielen.

Lemma 4.3 (Friedrichsche Ungleichung) Es sei $\Omega \subset \mathbb{R}^{d}$ ein beschränktes Gebiet mit Durchmesser $\operatorname{diam}(\Omega)=D<\infty$, dann gilt

$$
\|v\|_{0} \leq C_{\mathrm{F}}\|\nabla v\|_{0}=C_{\mathrm{F}}|v|_{1}
$$

für alle $v \in H_{0}^{1}(\Omega)$ mit $C_{\mathrm{F}} \leq 2 D$.

Da die Variationsgleichungen keine Kontrolle über die Fehler in der $L^{2}$-Norm liefert, können diese Terme mittels der Friedrichschen Ungleichung über die Seminorm abgeschätzt werden.

Lemma 4.4 (Kornsche Ungleichung) Es gibt eine nur vom Gebiet $\Omega$ abhängige Konstante $C_{\text {Korn }}$, so dass

$$
\|\nabla \mathbf{u}\| \leq C_{\text {Korn }}\|\mathbb{D} \mathbf{u}\|_{0}
$$

für alle $\mathbf{u} \in H_{0}^{1}(\Omega)$ gilt.

Für die Konvektionsterme zitieren wir aus [43] die Teile (e) und (f) des Lemma 2.2. 
Lemma 4.5 Das Gebiet $\Omega \subset \mathbb{R}^{d}$ erfülle die starke Lipschitzbedingung von Adams (siehe Seite 66 in [1]). Die Trilinearform $b(\cdot, \cdot, \cdot)$ entspreche der konvektiven Form $b=b_{c}$, der konservativen Form oder der schiefsymmetrischen Form $b=b_{s}$. Dann gelten die Abschätzungen

$$
\begin{aligned}
& \text { (e) }|b(\mathbf{w}, \mathbf{u}, \mathbf{v})| \leq C\|\nabla \mathbf{w}\|_{0}\|\nabla \mathbf{u}\|_{0}\|\nabla \mathbf{v}\|_{0}, \\
& \text { (f) }|b(\mathbf{w}, \mathbf{u}, \mathbf{v})| \leq C\|\mathbf{w}\|_{0}^{1-s}\|\nabla \mathbf{w}\|_{0}^{s}\|\nabla \mathbf{u}\|_{0}\|\nabla \mathbf{v}\|_{0}
\end{aligned}
$$

für alle $\mathbf{w}, \mathbf{u}, \mathbf{v} \in \mathbf{V}$ mit $0<s \leq 1$ für die Raumdimension $d=2$ und mit $s=\frac{1}{2}$ für $d=3$.

Diese Abschätzungen basieren im Wesentlichen auf der Hölderschen Ungleichung und Sobolevschen Einbettungssätzen.

Korollar 4.6 Die Abschätzungen lassen sich mit Hilfe der Korn-Ungleichung (4.7) in die im Folgenden benötigte Form bringen:

$$
\begin{aligned}
& |b(\mathbf{w}, \mathbf{u}, \mathbf{v})| \leq C_{\mathrm{LT}}\|\mathbf{w}\|_{0}^{\frac{1}{2}}\|\mathbb{D} \mathbf{w}\|_{0}^{\frac{1}{2}}\|\mathbb{D} \mathbf{u}\|_{0}\|\mathbb{D} \mathbf{v}\|_{0}, \\
& |b(\mathbf{w}, \mathbf{u}, \mathbf{v})| \leq C_{\mathrm{LT}}\|\mathbb{D} \mathbf{w}\|_{0}\|\mathbb{D} \mathbf{u}\|_{0}\|\nabla \mathbf{v}\|_{0} .
\end{aligned}
$$

Während die Cauchy-Schwarzsche Ungleichung zellweise angewendet werden kann und die Resulate somit lokal sind, handelt es sich bei der Friedrichschen und der Kornschen Ungleichung um globale Abschätzungen, die sich wegen der Verwendung von Randbedingungen nicht lokalisieren lassen.

Im Folgenden zitieren wir die Gronwallsche Ungleichung in einer für die folgenden Untersuchungen geeigneten Form. Sie wird es uns an verschiedenen Stellen dieses Kapitels ermöglichen, implizite Abschätzungen von Ableitungen in explizite Abschätzungen zu überführen.

Lemma 4.7 (Gronwallsche Ungleichung) Es seien $g_{i} \in L^{1}(0, T), i \in\{1,2,3\}$ und $\int_{0}^{t} g_{1}(s) \mathrm{d} s, \int_{0}^{t} g_{2}(s) \mathrm{d} s$ stetig, nicht fallend auf $[0, T]$ sowie $g_{3}$ nicht negativ. Außerdem gelte

$$
\partial_{t}\left(\left\|\mathbf{e}_{h}^{u}\right\|_{0}^{2}+\left\|e_{h}^{\theta}\right\|_{0}^{2}\right)+g_{1}(t) \leq g_{2}(t)+g_{3}(t)\left(\left\|\mathbf{e}_{h}^{u}\right\|_{0}^{2}+\left\|e_{h}^{\theta}\right\|_{0}^{2}\right) \quad \forall t \in[0, T] .
$$

Dann gilt auch

$$
\begin{aligned}
\left\|\mathbf{e}_{h}^{u}(t)\right\|_{0}^{2}+\left\|e_{h}^{\theta}(t)\right\|_{0}^{2}+\int_{0}^{t} & g_{1}(s) \mathrm{d} s \\
& \leq \exp \left(\int_{0}^{t} g_{3}(s) \mathrm{d} s\right)\left(\left\|\mathbf{e}_{h}^{u}(0)\right\|_{0}^{2}+\left\|e_{h}^{\theta}(0)\right\|_{0}^{2}+\int_{0}^{t} g_{2}(s) \mathrm{d} s\right)
\end{aligned}
$$

für alle $t \in[0, T]$, siehe [60]. 


\subsection{Eindeutigkeit und Stabilitätsabschätzungen}

Zur Charakterisierung der zeitlichen Regularität von Lösungen verwenden wir die Räume $L^{p}(0, t ; X)$. Diese sind definiert als

$$
L^{p}(a, b ; X)=\left\{f:[a, b] \rightarrow X \mid f \text { messbar, } \int_{a}^{b}\|f(s)\|_{X}^{p} \mathrm{~d} s<\infty\right\}
$$

für $1 \leq p<\infty$ und

$$
L^{\infty}(a, b ; X)=\left\{f:[a, b] \rightarrow X \mid f \text { messbar, ess } \sup _{s \in[a, b]}\|f(s)\|_{X}<\infty\right\}
$$

für $p=\infty$.

Annahme: Die kontinuierliche Lösung $\mathbf{u}, \theta$ und die diskrete Lösung $\mathbf{u}_{h}, \theta_{h}$ erfüllen folgende Regularitätsanforderungen:

$$
\begin{array}{rlrl}
\partial_{t} \mathbf{u}_{(h)} & \in L^{2}\left(0, t ; H^{-1}(\Omega)^{d}\right), & & \partial_{t} \theta_{(h)} \in L^{2}\left(0, t ; H^{-1}(\Omega)\right), \\
\mathbb{D} \mathbf{u}_{(h)} \in L^{4}\left(0, t ; L^{2}(\Omega)^{d \times d}\right), & \nabla \theta_{(h)} \in L^{4}\left(0, t ; L^{2}(\Omega)^{d}\right) .
\end{array}
$$

Lemma 4.8 Die Lösungen $\mathbf{u}_{h}, \theta_{h}$ von (4.1) und (4.2) sind unter Annahme der Regularität (4.14) eindeutig bestimmt.

Beweis: Es seien $\left(\mathbf{u}_{h, i}, \theta_{h, i}\right)$ für $i=1,2$ zwei Lösungen von (4.1) und (4.2) sowie $\mathbf{d}_{u}:=\mathbf{u}_{h, 1}-\mathbf{u}_{h, 2}, d_{\theta}:=\theta_{h, 1}-\theta_{h, 2}$. Durch Subtraktion der Variationsgleichungen für beide Lösungen mit den Differenzen $\mathbf{d}_{u}$ und $d_{\theta}$ als Testfunktionen erhalten wir die Differenzengleichungen

$$
\begin{aligned}
\left(\partial_{t} \mathbf{d}_{u}, \mathbf{d}_{u}\right)+\left(2 \nu \mathbb{D} \mathbf{d}_{u}, \mathbb{D} \mathbf{d}_{u}\right) & +\sum_{K \in \mathcal{T}_{h}}\left[\nu_{K}^{T}\left(\kappa_{u}\left(\mathbb{D} \mathbf{d}_{u}\right), \kappa_{u}\left(\mathbb{D} \mathbf{d}_{u}\right)\right)_{K}+\gamma_{K}\left(\nabla \cdot \mathbf{d}_{u}, \nabla \cdot \mathbf{d}_{u}\right)_{K}\right] \\
& =-\left(\beta \mathbf{g} d_{\theta}, \mathbf{d}_{u}\right)-b_{s}\left(\mathbf{u}_{h, 1}, \mathbf{u}_{h, 1}, \mathbf{d}_{u}\right)+b_{s}\left(\mathbf{u}_{h, 2}, \mathbf{u}_{h, 2}, \mathbf{d}_{u}\right) \\
& =-\left(\beta \mathbf{g} d_{\theta}, \mathbf{d}_{u}\right)-b_{s}\left(\mathbf{d}_{u}, \mathbf{u}_{h, 1}, \mathbf{d}_{u}\right)
\end{aligned}
$$

und

$$
\begin{aligned}
\left(\partial_{t} d_{\theta}, d_{\theta}\right)+\left(\alpha \nabla d_{\theta}, \nabla d_{\theta}\right) & +\sum_{K \in \mathcal{T}_{h}} \alpha_{K}^{T}\left(\kappa_{\theta}\left(\nabla d_{\theta}\right), \kappa_{\theta}\left(\nabla d_{\theta}\right)\right) \\
& =-c_{s}\left(\mathbf{u}_{h, 1}, \theta_{h, 1}, d_{\theta}\right)+c_{s}\left(\mathbf{u}_{h, 2}, \theta_{h, 2}, d_{\theta}\right)=-c_{s}\left(\mathbf{d}_{u}, \theta_{h, 1}, d_{\theta}\right) .
\end{aligned}
$$

Aus diesen beiden Gleichungen folgt mittels der Abschätzungen aus dem vorherigen Abschnitt

$$
\begin{aligned}
\frac{1}{2} \partial_{t}\left\|\mathbf{d}_{u}\right\|_{0}^{2}+\nu\left\|\mathbb{D} \mathbf{d}_{u}\right\|_{0}^{2} & +\sum_{K \in \mathcal{T}_{h}}\left[\nu_{K}^{T}\left\|\kappa_{u}\left(\mathbb{D} \mathbf{d}_{u}\right)\right\|_{L^{2}(K)}^{2}+\gamma_{K}\left\|\nabla \cdot \mathbf{d}_{u}\right\|_{L^{2}(K)}^{2}\right] \\
& \leq C_{\mathrm{LT}}\left\|\mathbb{D} \mathbf{u}_{h, 1}\right\|_{0}\left\|\mathbf{d}_{u}\right\|_{0}^{1 / 2}\left\|\mathbb{D} \mathbf{d}_{u}\right\|_{0}^{3 / 2}-\nu\left\|\mathbb{D} \mathbf{d}_{u}\right\|_{0}^{2}+\beta\|\mathbf{g}\|_{\infty}\left\|d_{\theta}\right\|_{0}\left\|\mathbf{d}_{u}\right\|_{0} \\
& \leq \frac{27 C_{\mathrm{LT}}^{4}}{64 \nu^{3}}\left\|\mathbb{D} \mathbf{u}_{h, 1}\right\|_{0}^{4}\left\|\mathbf{d}_{u}\right\|_{0}^{2}+\frac{\beta\|\mathbf{g}\|_{\infty}}{2}\left(\left\|d_{\theta}\right\|_{0}^{2}+\left\|\mathbf{d}_{u}\right\|_{0}^{2}\right)
\end{aligned}
$$


und

$$
\begin{aligned}
\frac{1}{2} \partial_{t}\left\|d_{\theta}\right\|_{0}^{2} & +\frac{1}{2} \alpha\left\|\nabla d_{\theta}\right\|_{0}^{2}+\sum_{K \in \mathcal{T}_{h}} \alpha_{K}^{T}\left\|\kappa_{\theta}\left(\nabla d_{\theta}\right)\right\|_{L^{2}(K)}^{2} \\
& \leq C_{\mathrm{LT}}\left\|\mathbf{d}_{u}\right\|_{0}^{1 / 2}\left\|\mathbb{D} \mathbf{d}_{u}\right\|_{0}^{1 / 2}\left\|\nabla \theta_{h, 1}\right\|_{0}\left\|\nabla d_{\theta}\right\|_{0}-\frac{1}{2} \alpha\left\|\nabla d_{\theta}\right\|_{0}^{2} \\
& \leq \frac{C_{\mathrm{LT}}^{2}}{2 \alpha}\left\|\mathbf{d}_{u}\right\|_{0}\left\|\mathbb{D} \mathbf{d}_{u}\right\|_{0}\left\|\nabla \theta_{h, 1}\right\|_{0}^{2} \leq \frac{C_{\mathrm{LT}}^{4}}{8 \alpha^{2} \nu}\left\|\nabla \theta_{h, 1}\right\|_{0}^{4}\left\|\mathbf{d}_{u}\right\|_{0}^{2}+\frac{1}{2} \nu\left\|\mathbb{D} \mathbf{d}_{u}\right\|_{0}^{2} .
\end{aligned}
$$

Nach Addition beider Ungleichungen ist die Anwendung der Gronwallschen Ungleichung möglich. Die Voraussetzungen an $g_{3}$ sind dank der Regularitätsanforderungen erfüllt. Wegen $g_{2}=0$ und $\left\|\mathbf{d}_{u}(0)\right\|=\left\|d_{\theta}(0)\right\|=0$ folgt

$$
\begin{aligned}
& \left\|\mathbf{d}_{u}(t)\right\|_{0}^{2}+\left\|d_{\theta}(t)\right\|_{0}^{2}+\int_{0}^{t}\left(\nu\left\|\mathbb{D} \mathbf{d}_{u}\right\|_{0}^{2}+\alpha\left\|\nabla d_{\theta}\right\|_{0}^{2}\right) \mathrm{d} s \\
& \quad+2 \int_{0}^{t} \sum_{K \in \mathcal{T}_{h}}\left[\nu_{K}^{T}\left\|\kappa_{u}\left(\mathbb{D} \mathbf{d}_{u}\right)\right\|_{L^{2}(K)}^{2}+\gamma_{K}\left\|\nabla \cdot \mathbf{d}_{u}\right\|_{L^{2}(K)}^{2}+\alpha_{K}^{T}\left\|\kappa_{\theta}\left(\nabla d_{\theta}\right)\right\|_{L^{2}(K)}^{2}\right] \mathrm{d} s=0 .
\end{aligned}
$$

Da alle Terme der linken Seite nicht-negativ sind folgt daraus die Behauptung $\mathbf{u}_{h, 1}=\mathbf{u}_{h, 2}$ und $\theta_{h, 1}=\theta_{h, 2}$.

Bemerkung 4.1 Der Eindeutigkeitsbeweis für die diskrete Lösung lässt sich unter den gleichen Regularitätsanforderungen auch für die kontinuierliche Lösung wiederholen. Die Einfachheit dieses Beweises lässt sich mit den hohen Regularitätsanforderungen begründen. Da das Ziel dieses Kapitels eine a-priori Fehlerabschätzung ist, deren Ordnung von Interpolationsabschätzungen abhängt, die noch deutlich höhere Regularitätsanforderungen besitzen, stellt das an dieser Stelle jedoch keine besondere Einschränkung dar.

Das folgende Korollar liefert Regularitätsaussagen über die Lösung, die sich aus den Variationsgleichungen herleiten lassen.

Korollar 4.9 Die äußeren Kraftterme der rechten Seiten f, $Q$ und die Startwerte erfüllen

$$
\begin{aligned}
\mathbf{f} & \in L^{1}\left(0, t ; L^{2}(\Omega)^{d}\right), & \mathbf{u}_{h}^{0} & \in L^{2}(\Omega)^{d}, \\
Q & \in L^{1}\left(0, t ; L^{2}(\Omega)\right), & \theta_{h}^{0} & \in L^{2}(\Omega),
\end{aligned}
$$

dann gilt für die Lösung $\mathbf{u}_{h}, \theta_{h}$ von (4.1) und (4.2)

$$
\begin{aligned}
\mathbf{u}_{h} & \in L^{\infty}\left(0, t ; L^{2}(\Omega)^{d}\right), & & \mathbb{D} \mathbf{u}_{h} \in L^{2}\left(0, t ; L^{2}(\Omega)^{d \times d}\right), \\
\theta_{h} & \in L^{\infty}\left(0, t ; L^{2}(\Omega)\right), & & \nabla \theta_{h} \in L^{2}\left(0, t ; L^{2}(\Omega)^{d}\right) .
\end{aligned}
$$

Diese Aussage folgt aus den Stabilitätsabschätzungen in den zugehörigen Normen, die auf die beiden folgenden Lemmata aufgeteilt sind. 
Lemma 4.10 (Energieabschätzungen) Die diskreten Lösungen $\mathbf{u}_{h}, \theta_{h}$ von (4.1) und (4.2) erfüllen folgende Stabilitätsabschätzungen in der Energienorm:

$$
\begin{aligned}
\left\|\theta_{h}\right\|_{L^{\infty}\left(0, t ; L^{2}(\Omega)\right)} & \leq\left\|\theta_{h}^{0}\right\|_{0}+\|Q\|_{L^{1}\left(0, t ; L^{2}(\Omega)\right)} \\
& =: K_{1}\left(t, \theta_{h}^{0}, Q\right), \\
\left\|\mathbf{u}_{h}\right\|_{L^{\infty}\left(0, t ; L^{2}(\Omega)\right)} & \leq\left\|\mathbf{u}_{h}^{0}\right\|_{0}+\|\mathbf{f}\|_{L^{1}\left(0, t ; L^{2}(\Omega)\right)}+C \alpha\|\mathbf{g}\|_{\infty} K_{1}\left(\theta_{h}^{0}, Q\right) \\
& =: K_{2}\left(t, \mathbf{u}_{h}^{0}, \mathbf{f}, \theta_{h}^{0}, Q\right) .
\end{aligned}
$$

Beweis: Die Energieabschätzungen lassen sich einfach aus den Variationsgleichungen (4.1) und (4.2) durch Verwendung von $\theta_{h}$ und $\mathbf{u}_{h}$ als Testfunktionen gewinnen. Es gilt

$$
\frac{1}{2} \partial_{t}\left\|\theta_{h}\right\|_{0}^{2}+\alpha\left\|\nabla \theta_{h}\right\|_{0}^{2}+\sum_{K \in \mathcal{T}_{h}} \alpha_{K}^{T}\left\|\kappa_{\theta}\left(\nabla \theta_{h}\right)\right\|_{L^{2}(K)}^{2}=\left(Q, \theta_{h}\right)
$$

und

$$
\begin{aligned}
\frac{1}{2} \partial_{t}\left\|\mathbf{u}_{h}\right\|_{0}^{2}+2 \nu\left\|\mathbb{D} \mathbf{u}_{h}\right\|_{0}^{2}+\sum_{K \in \mathcal{T}_{h}}\left(\nu_{K}^{T}\left\|\kappa_{u}\left(\mathbb{D} \mathbf{u}_{h}\right)\right\|_{L^{2}(K)}^{2}+\gamma_{K}\left\|\nabla \cdot \mathbf{u}_{h}\right\|_{L^{2}(K)}^{2}\right) \\
=\left(\mathbf{f}-\beta \theta_{h} \mathbf{g}, \mathbf{u}_{h}\right) .
\end{aligned}
$$

Da die schiefsymmetrischen Konvektionsterme beim symmetrischen Einsetzen verschwinden, hängt die Temperaturabschätzung nicht mehr von der Geschwindigkeit ab. Aus (4.21) folgt wegen $\alpha, \alpha_{K}^{T} \geq 0$ und mittels der Cauchy-Schwarzschen Ungleichung

$$
\frac{1}{2} \partial_{t}\left\|\theta_{h}\right\|_{0}^{2}=\left\|\theta_{h}\right\|_{0} \partial_{t}\left\|\theta_{h}\right\|_{0} \leq\left(Q, \theta_{h}\right) \leq\|Q\|_{0}\left\|\theta_{h}\right\|_{0}
$$

und damit $\partial_{t}\left\|\theta_{h}\right\|_{0} \leq\|Q\|_{0}$. Durch Integration über das Intervall $(0, t)$ folgt der erste Teil der Behauptung. Aus der zweiten Gleichung (4.22) folgt wegen $\nu, \nu_{K}^{T}, \gamma_{K} \geq 0$ analog

$$
\frac{1}{2} \partial_{t}\left\|\mathbf{u}_{h}\right\|_{0}^{2}=\left\|\mathbf{u}_{h}\right\|_{0} \partial_{t}\left\|\mathbf{u}_{h}\right\|_{0} \leq\left(\mathbf{f}-\beta \theta_{h} \mathbf{g}, \mathbf{u}_{h}\right) \leq\left(\|\mathbf{f}\|_{0}+\beta\|\mathbf{g}\|_{\infty}\left\|\theta_{h}\right\|_{0}\right)\left\|\mathbf{u}_{h}\right\|_{0}
$$

und damit $\partial_{t}\left\|\mathbf{u}_{h}\right\|_{0} \leq\|\mathbf{f}\|_{0}+\beta\|\mathbf{g}\|_{\infty}\left\|\theta_{h}\right\|_{0}$, woraus wiederum nach Integration über $(0, t)$ und unter Verwendung der vorherigen Abschätzung der zweite Teil der Behauptung folgt.

Ähnlich zu den Energieabschätzungen lassen sich auch die Diffusionsterme und die zum Turbulenzmodell gehörenden Terme abschätzen.

Lemma 4.11 Für die diskreten Lösungen $\mathbf{u}_{h}, \theta_{h}$ von (4.1) und (4.2) gilt:

$$
\begin{aligned}
\alpha\left\|\nabla \theta_{h}\right\|_{L^{2}\left(0, t ; L^{2}(\Omega)\right)}^{2}+\int_{0}^{t} \sum_{K \in \mathcal{T}_{h}} \alpha_{K}^{T}\left\|\kappa_{\theta}\left(\nabla \theta_{h}\right)\right\|_{L^{2}(K)}^{2} \mathrm{~d} s & \leq K_{1}\left(t, \theta_{h}^{0}, Q\right)^{2}, \\
\nu\left\|\mathbb{D} \mathbf{u}_{h}\right\|_{L^{2}\left(0, t ; L^{2}(\Omega)\right)}^{2}+\frac{1}{2} \int_{0}^{t} \sum_{K \in \mathcal{T}_{h}}\left(\nu_{K}^{T}\left\|\kappa_{u}\left(\mathbb{D} \mathbf{u}_{h}\right)\right\|_{L^{2}(K)}^{2}\right. & \left.+\gamma_{K}\left\|\nabla \cdot \mathbf{u}_{h}\right\|_{L^{2}(K)}^{2}\right) \mathrm{d} s \\
& \leq K_{2}\left(t, \mathbf{u}_{h}^{0}, \mathbf{f}, \theta_{h}^{0}, Q\right)^{2} .
\end{aligned}
$$


Beweis: Der erste Teil folgt wiederum aus (4.21) durch Integration über $(0, t)$ und Ausnutzung der bisherigen Abschätzung:

$$
\begin{gathered}
\alpha\left\|\nabla \theta_{h}\right\|_{L^{2}\left(0, t ; L^{2}(\Omega)\right)}^{2}+\int_{0}^{t} \sum_{K \in \mathcal{T}_{h}} \alpha_{K}^{T}\left\|\kappa_{\theta}\left(\nabla \theta_{h}\right)\right\|_{L^{2}(K)}^{2} \mathrm{~d} s \leq \int_{0}^{t}\left(Q, \theta_{h}\right) \mathrm{d} s \\
\leq \int_{0}^{t}\|Q\|_{0}\left\|\theta_{h}\right\|_{0} \mathrm{~d} s \leq K_{1}\left(t, \theta_{h}^{0}, Q\right) \int_{0}^{t}\|Q\|_{0} \mathrm{~d} s \leq K_{1}\left(t, \theta_{h}^{0}, Q\right)^{2} .
\end{gathered}
$$

Der zweite Teil folgt analog aus Gleichung (4.22):

$$
\begin{aligned}
2 \nu\left\|\mathbb{D} \mathbf{u}_{h}\right\|_{L^{2}\left(0, t ; L^{2}(\Omega)\right)}^{2}+\int_{0}^{t} \sum_{K \in \mathcal{T}_{h}}\left(\nu_{K}^{T}\left\|\kappa_{u}\left(\mathbb{D} \mathbf{u}_{h}\right)\right\|_{L^{2}(K)}^{2}+\gamma_{K}\left\|\nabla \cdot \mathbf{u}_{h}\right\|_{L^{2}(K)}^{2}\right) \mathrm{d} s \\
\leq \int_{0}^{t}\left(\mathbf{f}-\alpha \mathbf{g} \theta_{h}, \mathbf{u}_{h}\right) \mathrm{d} s \leq \int_{0}^{t}\left\|\mathbf{f}-\alpha \mathbf{g} \theta_{h}\right\|_{0}\left\|\mathbf{u}_{h}\right\|_{0} \mathrm{~d} s \\
\leq K_{2}\left(\mathbf{u}_{h}^{0}, \mathbf{f}, \theta_{h}^{0}, Q\right) \int_{0}^{t}\left(\|\mathbf{f}\|_{0}+\alpha\|\mathbf{g}\|_{\infty}\left\|\theta_{h}\right\|_{0}\right) \mathrm{d} s \leq K_{2}\left(\mathbf{u}_{h}^{0}, \mathbf{f}, \theta_{h}^{0}, Q\right)^{2} .
\end{aligned}
$$

Bemerkung 4.2 Fast identische Abschätzungen zu Lemma 4.10 und Lemma 4.11, bei denen lediglich die Turbulenzmodell-Terme fehlen, lassen sich auf die gleiche Weise für die exakte Lösung $\mathbf{u}, \theta$ aus den Variationsgleichungen (2.10) und (2.11) herleiten.

\subsection{A-priori Fehlerabschätzung für Geschwindigkeit und Temperatur}

Zunächst seien für $t \in[0, T]$ die Abbildungen $\|\cdot\| \|_{0, t}:([0, T] \rightarrow \mathbf{V}) \rightarrow \mathbb{R}$ und $|[\cdot]|_{0, t}:([0, T] \rightarrow \Theta) \rightarrow \mathbb{R}$ durch

$$
\begin{aligned}
\|\mathbf{v}\|_{0, t}^{2} & +|[\vartheta]|_{0, t}^{2}:=\|\mathbf{v}(t)\|_{0}^{2}+\|\vartheta(t)\|_{0}^{2}+\nu\|\mathbb{D} \mathbf{v}\|_{L^{2}\left(0, t, L^{2}(\Omega)\right)}^{2}+\alpha\|\nabla \vartheta\|_{L^{2}\left(0, t, L^{2}(\Omega)\right)}^{2} \\
& +\int_{0}^{t} \sum_{K \in \mathcal{T}_{h}}\left(\nu_{K}^{T}\left\|\kappa_{u}(\mathbb{D} \mathbf{v}(s))\right\|_{0, K}^{2}+\gamma_{K}\|\nabla \cdot \mathbf{v}(s)\|_{0, K}^{2}+\alpha_{K}^{T}\left\|\kappa_{\theta}(\nabla \vartheta(s))\right\|_{0, K}^{2}\right) \mathrm{d} s
\end{aligned}
$$

definiert und darauf hingewiesen, dass es sich wegen der Abhängigkeit der Parameter $\nu_{K}^{T}, \gamma_{K}, \alpha_{K}^{T}$ von der diskreten Lösung nicht um eine Norm handelt. Die Regularität von v und $\vartheta$ entspreche dabei der Regularität der Lösung (4.14), (4.17) und (4.18).

Satz 4.12 Es sei $\mathbf{u}, \theta$ die kontinuierliche Lösung von (2.10), (2.11) und $\mathbf{u}_{h}, \theta_{h}$ die diskrete Lösung von (4.1), (4.2). Weiterhin gelten alle bisher genannten Regularitätsannahmen. 
Dann gilt:

$$
\begin{aligned}
& \frac{1}{2}\left\|\mathbf{u}-\mathbf{u}_{h}\right\|_{0, t}^{2}+\frac{1}{2}\left|\left[\theta-\theta_{h}\right]\right|_{0, t}^{2} \leq \inf _{\tilde{\mathbf{u}}_{h} \in L^{2}\left(0, t ; \mathbf{Z}_{h}\right)}\left\|\mathbf{u}-\tilde{\mathbf{u}}_{h}\right\|_{0, t}^{2}+\underset{\tilde{\theta}_{h} \in L^{2}\left(0, t ; \Theta_{h}\right)}{\inf }\left|\left[\theta-\tilde{\theta}_{h}\right]\right|_{0, t}^{2} \\
& +\exp \left(\int_{0}^{t} \frac{27 C_{\mathrm{LT}}^{4}}{2 \nu^{3}}\|\mathbb{D} \mathbf{u}\|_{0}^{4}+\frac{6 C_{1}^{4}}{\nu \alpha^{2}}\|\nabla \theta\|_{0}^{4}+2 \beta\|\mathbf{g}\|_{\infty} \mathrm{d} s\right) \\
& \inf _{\tilde{\mathbf{u}}_{h} \in L^{2}\left(0, t ; \mathbf{Z}_{h}\right)}\left(\left\|\left(\mathbf{u}-\tilde{\mathbf{u}}_{h}\right)(0)\right\|_{0}^{2}+\left\|\left(\theta-\tilde{\theta}_{h}\right)(0)\right\|_{0}^{2}+\int_{0}^{t} g_{2}(s) \mathrm{d} s\right) \\
& \tilde{\theta}_{h} \in L^{2}\left(0, t ; \Theta_{h}\right) \\
& \lambda_{h} \in L^{2}\left(0, t ; Q_{h}\right)
\end{aligned}
$$

für alle $t \in[0, T]$, wobei sich $g_{2}$, wie später in (4.31) definiert, aus weiteren Approximations- und Konsistenzfehlern zusammensetzt.

Beweis: Wir betrachten zunächst beliebige Approximationen $\tilde{\mathbf{u}}_{h}:[0, T] \rightarrow \mathbf{Z}_{h}$ und $\tilde{\theta}_{h}:[0, T] \rightarrow \Theta_{h}$ der exakten Lösung $\mathbf{u}, \theta$ und zerlegen die Fehler in Modellfehler $\mathbf{e}_{h}^{u}, e_{h}^{\theta}$ und Approximationsfehler $\epsilon^{u}, \epsilon^{\theta}$ :

$$
\begin{gathered}
\mathbf{u}_{h}-\mathbf{u}=\left(\mathbf{u}_{h}-\tilde{\mathbf{u}}_{h}\right)-\left(\mathbf{u}-\tilde{\mathbf{u}}_{h}\right)=: \mathbf{e}_{h}^{u}-\epsilon^{u}, \\
\theta_{h}-\theta=\left(\theta_{h}-\tilde{\theta}_{h}\right)-\left(\theta-\tilde{\theta}_{h}\right)=: e_{h}^{\theta}-\epsilon^{\theta} .
\end{gathered}
$$

Subtraktion der beiden Variationsgleichungen für $\mathbf{u}$ und $\mathbf{u}_{h}$ mit Testfunktionen $\mathbf{e}_{h}^{u}$ liefert

$$
\begin{aligned}
& \frac{1}{2} \partial_{t}\left\|\mathbf{e}_{h}^{u}\right\|_{0}^{2}+2 \nu\left\|\mathbb{D} \mathbf{e}_{h}^{u}\right\|_{0}^{2}+ \sum_{K \in \mathcal{T}_{h}}\left(\nu_{K}^{T}\left\|\kappa_{u}\left(\mathbb{D} \mathbf{e}_{h}^{u}\right)\right\|_{0, K}^{2}+\gamma_{K}\left\|\nabla \cdot \mathbf{e}_{h}^{u}\right\|_{0, K}^{2}\right) \\
&=\left(\partial_{t} \epsilon^{u}, \mathbf{e}_{h}^{u}\right)+2 \nu\left(\mathbb{D} \epsilon^{u}, \mathbb{D} \mathbf{e}_{h}^{u}\right)+b_{S}\left(\mathbf{u}, \mathbf{u}, \mathbf{e}_{h}^{u}\right)-b_{S}\left(\mathbf{u}_{h}, \mathbf{u}_{h}, \mathbf{e}_{h}^{u}\right)-\left(p-\lambda_{h}, \nabla \cdot \mathbf{e}_{h}^{u}\right) \\
&+\sum_{K \in \mathcal{T}_{h}} \nu_{K}^{T}\left(\kappa_{u}\left(\mathbb{D} \epsilon^{u}\right), \kappa_{u}\left(\mathbb{D} \mathbf{e}_{h}^{u}\right)\right)_{K}-\sum_{K \in \mathcal{T}_{h}} \nu_{K}^{T}\left(\kappa_{u}(\mathbb{D} \mathbf{u}), \kappa_{u}\left(\mathbb{D} \mathbf{e}_{h}^{u}\right)\right)_{K} \\
&+\sum_{K \in \mathcal{T}_{h}} \gamma_{K}\left(\nabla \cdot \epsilon^{u}, \nabla \cdot \mathbf{e}_{h}^{u}\right)_{K}-\alpha\left(\mathbf{g} e_{h}^{\theta}, \mathbf{e}_{h}^{u}\right)+\alpha\left(\mathbf{g} \epsilon^{\theta}, \mathbf{e}_{h}^{u}\right)
\end{aligned}
$$

Wegen $\mathbf{e}_{h}^{u} \in \mathbf{Z}_{h}$ kann dabei ein beliebiger diskreter Druck $\lambda_{h}:[0, T] \rightarrow Q_{h}$ als Approximation an den exakten Druck $p$ verwendet werden. Analog folgt für $e_{h}^{\theta}$

$$
\begin{aligned}
\frac{1}{2} \partial_{t}\left\|e_{h}^{\theta}\right\|_{0}^{2}+\alpha & \left\|\nabla e_{h}^{\theta}\right\|_{0}^{2}+\sum_{K \in \mathcal{T}_{h}} \alpha_{K}^{T}\left\|\kappa_{\theta}\left(\nabla e_{h}^{\theta}\right)\right\|_{0, K}^{2} \\
& =\left(\partial_{t} \epsilon^{\theta}, e_{h}^{\theta}\right)+\alpha\left(\nabla \epsilon^{\theta}, \nabla e_{h}^{\theta}\right)+c_{S}\left(\mathbf{u}, \theta, e_{h}^{\theta}\right)-c_{S}\left(\mathbf{u}_{h}, \theta_{h}, e_{h}^{\theta}\right) \\
& +\sum_{K \in \mathcal{T}_{h}} \alpha_{K}^{T}\left(\kappa_{\theta}\left(\nabla \epsilon^{\theta}\right), \kappa_{\theta}\left(\nabla e_{h}^{\theta}\right)\right)_{K}-\sum_{K \in \mathcal{T}_{h}} \alpha_{K}^{T}\left(\kappa_{\theta}(\nabla \theta), \kappa_{\theta}\left(\nabla e_{h}^{\theta}\right)\right)_{K} .
\end{aligned}
$$


Die Aufgabe wird zunächst sein, alle Terme der rechten Seiten von (4.26) und (4.27) einzeln abzuschätzen. Wir beginnen mit der Impulsgleichung und erhalten:

$$
\begin{aligned}
\left(\partial_{t} \epsilon^{u}, \mathbf{e}_{h}^{u}\right) & \leq C_{\text {Korn }}\left\|\partial_{t} \epsilon^{u}\right\|_{H^{-1}(\Omega)}\left\|\mathbb{D} \mathbf{e}_{h}^{u}\right\|_{0} \leq \frac{3 C_{\text {Korn }}^{2}}{\nu}\left\|\partial_{t} \epsilon^{u}\right\|_{H^{-1}(\Omega)}^{2}+\frac{1}{12} \nu\left\|\mathbb{D} \mathbf{e}_{h}^{u}\right\|_{0}^{2}, \\
\left(2 \nu \mathbb{D} \epsilon^{u}, \mathbb{D} \mathbf{e}_{h}^{u}\right) & \leq 2 \nu\left\|\mathbb{D} \epsilon^{u}\right\|_{0}\left\|\mathbb{D} \mathbf{e}_{h}^{u}\right\|_{0} \leq 2 \nu\left\|\mathbb{D} \epsilon^{u}\right\|_{0}^{2}+\frac{\nu}{2}\left\|\mathbb{D} \mathbf{e}_{h}^{u}\right\|_{0}^{2} .
\end{aligned}
$$

Für die beiden Terme des Turbulenzmodells gilt:

$$
\begin{aligned}
& \sum_{K \in \mathcal{T}_{h}} \nu_{K}^{T}\left(\kappa_{u}\left(\mathbb{D} \epsilon^{u}\right), \kappa_{u}\left(\mathbb{D} \mathbf{e}_{h}^{u}\right)\right)_{K} \leq \sum_{K \in \mathcal{T}_{h}} \nu_{K}^{T}\left\|\kappa_{u}\left(\mathbb{D} \epsilon^{u}\right)\right\|_{L^{2}(K)}^{2}+\sum_{K \in \mathcal{T}_{h}} \frac{\nu_{K}^{T}}{4}\left\|\kappa_{u}\left(\mathbb{D} \mathbf{e}_{h}^{u}\right)\right\|_{L^{2}(K)}^{2}, \\
& \sum_{K \in \mathcal{T}_{h}} \nu_{K}^{T}\left(\kappa_{u}(\mathbb{D} \mathbf{u}), \kappa_{u}\left(\mathbb{D} \mathbf{e}_{h}^{u}\right)\right)_{K} \leq \sum_{K \in \mathcal{T}_{h}} \nu_{K}^{T}\left\|\kappa_{u}(\mathbb{D} \mathbf{u})\right\|_{L^{2}(K)}^{2}+\sum_{K \in \mathcal{T}_{h}} \frac{\nu_{K}^{T}}{4}\left\|\kappa_{u}\left(\mathbb{D} \mathbf{e}_{h}^{u}\right)\right\|_{L^{2}(K)}^{2} .
\end{aligned}
$$

Die Terme, die die Divergenz enthalten, können auf zwei Weisen abgeschätzt werden, einmal über den Divergenz-Stabilisierungsterm oder über den Diffusionsterm:

$$
\begin{aligned}
\left(p-\lambda_{h}, \nabla \cdot \mathbf{e}_{h}^{u}\right) & =\sum_{K \in \mathcal{T}_{h}}\left(p-\lambda_{h}, \nabla \cdot \mathbf{e}_{h}^{u}\right)_{K} \\
& \leq \sum_{K \in \mathcal{T}_{h}}\left\|p-\lambda_{h}\right\|_{L^{2}(K)}\left\|\nabla \cdot \mathbf{e}_{h}^{u}\right\|_{L^{2}(K)} \\
& \leq \sum_{K \in \mathcal{T}_{h}}\left[\frac{1}{\gamma_{K}}\left\|p-\lambda_{h}\right\|_{L^{2}(K)}^{2}+\frac{\gamma_{K}}{4}\left\|\nabla \cdot \mathbf{e}_{h}^{u}\right\|_{L^{2}(K)}^{2}\right]
\end{aligned}
$$

und

$$
\begin{aligned}
\left(p-\lambda_{h}, \nabla \cdot \mathbf{e}_{h}^{u}\right) & \leq\left\|p-\lambda_{h}\right\|_{0}\left\|\nabla \cdot \mathbf{e}_{h}^{u}\right\|_{0} \\
& \leq \sqrt{3} C_{\text {Korn }}\left\|p-\lambda_{h}\right\|_{0}\left\|\mathbb{D} \mathbf{e}_{h}^{u}\right\|_{0} \\
& \leq \frac{9 C_{\text {Korn }}^{2}}{\nu}\left\|p-\lambda_{h}\right\|_{0}^{2}+\frac{\nu}{12}\left\|\mathbb{D} \mathbf{e}_{h}^{u}\right\|_{0}^{2}
\end{aligned}
$$

sowie

$$
\begin{aligned}
\sum_{K \in \mathcal{T}_{h}} \gamma_{K}\left(\nabla \cdot \epsilon^{u}, \nabla \cdot \mathbf{e}_{h}^{u}\right)_{K} & \leq \sum_{K \in \mathcal{T}_{h}} \gamma_{K}\left\|\nabla \cdot \epsilon^{u}\right\|_{L^{2}(K)}\left\|\nabla \cdot \mathbf{e}_{h}^{u}\right\|_{L^{2}(K)} \\
& \leq \sum_{K \in \mathcal{T}_{h}}\left[\gamma_{K}\left\|\nabla \cdot \epsilon^{u}\right\|_{L^{2}(K)}^{2}+\frac{\gamma_{K}}{4}\left\|\nabla \cdot \mathbf{e}_{h}^{u}\right\|_{L^{2}(K)}^{2}\right]
\end{aligned}
$$

und

$$
\begin{aligned}
\sum_{K \in \mathcal{T}_{h}} \gamma_{K}\left(\nabla \cdot \epsilon^{u}, \nabla \cdot \mathbf{e}_{h}^{u}\right)_{K} & \leq \sum_{K \in \mathcal{T}_{h}} \gamma_{K}\left\|\nabla \cdot \epsilon^{u}\right\|_{L^{2}(K)}\left\|\nabla \cdot \mathbf{e}_{h}^{u}\right\|_{L^{2}(K)} \\
& \leq \sum_{K \in \mathcal{T}_{h}}\left[\frac{9 C_{\mathrm{Korn}}^{2}}{\nu} \gamma_{K}^{2}\left\|\nabla \cdot \epsilon^{u}\right\|_{L^{2}(K)}^{2}+\frac{\nu}{36 C_{\mathrm{Korn}}^{2}}\left\|\nabla \cdot \mathbf{e}_{h^{u}}^{u}\right\|_{L^{2}(K)}^{2}\right] \\
& \leq \sum_{K \in \mathcal{T}_{h}} \frac{9 C_{\mathrm{Korn}}^{2}}{\nu} \gamma_{K}^{2}\left\|\nabla \cdot \epsilon^{u}\right\|_{L^{2}(K)}^{2}+\frac{1}{12} \nu\left\|\mathbb{D} \mathbf{e}_{h}^{u}\right\|_{0}^{2} .
\end{aligned}
$$


Diese Abschätzungen lassen sich zu

$$
\begin{aligned}
\left(p-\lambda_{h}, \nabla \cdot \mathbf{e}_{h}^{u}\right) & \leq \sum_{K \in \mathcal{T}_{h}} \min \left(\frac{9 C_{\mathrm{Ko}}^{2}}{\nu}, \frac{1}{\gamma_{K}}\right)\left\|p-\lambda_{h}\right\|_{L^{2}(K)}^{2} \\
& +\frac{1}{12} \nu\left\|\mathbb{D} \mathbf{e}_{h}^{u}\right\|_{0}^{2}+\sum_{K \in \mathcal{T}_{h}} \frac{\gamma_{K}}{4}\left\|\nabla \cdot \mathbf{e}_{h}^{u}\right\|_{L^{2}(K)}^{2}, \\
\sum_{K \in \mathcal{T}_{h}} \gamma_{K}\left(\nabla \cdot \epsilon^{u}, \nabla \cdot \mathbf{e}_{h}^{u}\right)_{K} & \leq \sum_{K \in \mathcal{T}_{h}} \min \left(\frac{9 C_{\mathrm{Ko}}^{2}}{\nu}, \frac{1}{\gamma_{K}}\right) \gamma_{K}^{2}\left\|\nabla \cdot \epsilon^{u}\right\|_{L^{2}(K)}^{2} \\
& +\frac{1}{12} \nu\left\|\mathbb{D} \mathbf{e}_{h}^{u}\right\|_{0}^{2}+\sum_{K \in \mathcal{T}_{h}} \frac{\gamma_{K}}{4}\left\|\nabla \cdot \mathbf{e}_{h}^{u}\right\|_{L^{2}(K)}^{2}
\end{aligned}
$$

kombinieren. Der Divergenz-Stabilisierungsterm fängt also die Skalierung des Approximationsfehlers des Drucks mit dem Kehrwert der kinematischen Viskosität $\nu$ ab. Für $\gamma_{K} \gg \nu$ entspricht das einer deutlichen Verbesserung der Fehlerabschätzung.

Die nichtlinearen Konvektionsterme der Impulsgleichung werden wie folgt zerlegt:

$$
\begin{aligned}
b_{s}\left(\mathbf{u}, \mathbf{u}, \mathbf{e}_{h}^{u}\right) & -b_{s}\left(\mathbf{u}_{h}, \mathbf{u}_{h}, \mathbf{e}_{h}^{u}\right)=b_{s}\left(\mathbf{u}-\tilde{\mathbf{u}}_{h}+\tilde{\mathbf{u}}_{h}-\mathbf{u}_{h}+\mathbf{u}_{h}, \mathbf{u}, \mathbf{e}_{h}^{u}\right)-b_{s}\left(\mathbf{u}_{h}, \mathbf{u}_{h}-\mathbf{e}_{h}^{u}, \mathbf{e}_{h}^{u}\right) \\
& =b_{s}\left(\epsilon^{u}, \mathbf{u}, \mathbf{e}_{h}^{u}\right)-b_{s}\left(\mathbf{e}_{h}^{u}, \mathbf{u}, \mathbf{e}_{h}^{u}\right)+b_{s}\left(\mathbf{u}_{h}, \mathbf{u}, \mathbf{e}_{h}^{u}\right)-b_{s}\left(\mathbf{u}_{h}, \tilde{\mathbf{u}}_{h}, \mathbf{e}_{h}^{u}\right) \\
& =b_{s}\left(\epsilon^{u}, \mathbf{u}, \mathbf{e}_{h}^{u}\right)-b_{s}\left(\mathbf{e}_{h}^{u}, \mathbf{u}, \mathbf{e}_{h}^{u}\right)+b_{s}\left(\mathbf{u}_{h}, \epsilon^{u}, \mathbf{e}_{h}^{u}\right) .
\end{aligned}
$$

Durch diese Zerlegung wird erreicht, dass die diskrete Lösung $\mathbf{u}_{h}$ auf der rechten Seite der Fehlerabschätzung nur in Termen auftaucht, die durch die Stabilitätsabschätzung bereits abgeschätzt sind. Für die Abschätzung dieser Terme werden wir

$$
b_{S}(\mathbf{w}, \mathbf{u}, \mathbf{v}) \leq C_{\mathrm{LT}}\|\mathbf{w}\|_{0}^{\frac{1}{2}}\|\mathbb{D} \mathbf{w}\|_{0}^{\frac{1}{2}}\|\mathbb{D} \mathbf{u}\|_{0}\|\mathbb{D} \mathbf{v}\|_{0}
$$

aus Lemma 2.2 (f) in [43] und der Korn-Ungleichung verwenden. Der erste und der letzte Summand bereiten keine Probleme, da sie auch den Approximationsfehler $\epsilon^{u}$ enthalten. Für sie gilt

$$
\begin{aligned}
b_{s}\left(\epsilon^{u}, \mathbf{u}, \mathbf{e}_{h}^{u}\right) & \leq C_{\mathrm{LT}}\left\|\epsilon^{u}\right\|_{0}^{\frac{1}{2}}\left\|\mathbb{D} \epsilon^{u}\right\|_{0}^{\frac{1}{2}}\|\mathbb{D} \mathbf{u}\|_{0}\left\|\mathbb{D} \mathbf{e}_{h}^{u}\right\|_{0} \\
& \leq \frac{3 C_{\mathrm{LT}}^{2}}{\nu}\left\|\epsilon^{u}\right\|_{0}\left\|\mathbb{D} \epsilon^{u}\right\|_{0}\|\mathbb{D} \mathbf{u}\|_{0}^{2}+\frac{1}{12} \nu\left\|\mathbb{D} \mathbf{e}_{h}^{u}\right\|_{0}^{2}
\end{aligned}
$$

und

$$
\begin{aligned}
b_{s}\left(\mathbf{u}_{h}, \epsilon^{u}, \mathbf{e}_{h}^{u}\right) & \leq C_{\mathrm{LT}}\left\|\mathbf{u}_{h}\right\|_{0}^{\frac{1}{2}}\left\|\mathbb{D} \mathbf{u}_{h}\right\|_{0}^{\frac{1}{2}}\left\|\mathbb{D} \epsilon^{u}\right\|_{0}\left\|\mathbb{D} \mathbf{e}_{h}^{u}\right\|_{0} \\
& \leq \frac{3 C_{\mathrm{LT}}^{2}}{\nu}\left\|\mathbf{u}_{h}\right\|_{0}\left\|\mathbb{D} \mathbf{u}_{h}\right\|_{0}\left\|\mathbb{D} \epsilon^{u}\right\|_{0}^{2}+\frac{1}{12} \nu\left\|\mathbb{D} \mathbf{e}_{h}^{u}\right\|_{0}^{2} .
\end{aligned}
$$

Problematisch ist der mittlere Term $b_{S}\left(\mathbf{e}_{h}^{u}, \mathbf{u}, \mathbf{e}_{h}^{u}\right)$ bei dem sich $\mathbf{e}_{h}^{u}$ nicht komplett von den restlichen Termen trennen lässt. Dieser lässt sich zumindest durch

$$
\begin{aligned}
b_{s}\left(\mathbf{e}_{h}^{u}, \mathbf{u}, \mathbf{e}_{h}^{u}\right) & \leq C_{\mathrm{LT}}\left\|\mathbf{e}_{h}^{u}\right\|_{0}^{\frac{1}{2}}\|\mathbb{D} \mathbf{u}\|_{0}\left\|\mathbb{D} \mathbf{e}_{h}^{u}\right\|_{0}^{\frac{3}{2}} \\
& \leq \frac{27 C_{\mathrm{LT}}^{4}}{4 \nu^{3}}\|\mathbb{D} \mathbf{u}\|_{0}^{4}\left\|\mathbf{e}_{h}^{u}\right\|_{0}^{2}+\frac{1}{4} \nu\left\|\mathbb{D} \mathbf{e}_{h}^{u}\right\|_{0}^{2}
\end{aligned}
$$


abschätzen. Für die Auftriebsterme in (4.26) erhalten wir

$$
\begin{aligned}
-\beta\left(\mathbf{g} e_{h}^{\theta}, \mathbf{e}_{h}^{u}\right)+\beta\left(\mathbf{g} \epsilon^{\theta}, \mathbf{e}_{h}^{u}\right) & \leq \beta\|\mathbf{g}\|_{\infty}\left(\left\|e_{h}^{\theta}\right\|+\left\|\epsilon^{\theta}\right\|\right)\left\|\mathbf{e}_{h}^{u}\right\|_{0} \\
& \leq \beta\|\mathbf{g}\|_{\infty}\left(\left\|\mathbf{e}_{h}^{u}\right\|_{0}^{2}+\left\|e_{h}^{\theta}\right\|_{0}^{2}\right)+\frac{\beta\|\mathbf{g}\|_{\infty}}{3}\left\|\epsilon^{\theta}\right\|_{0}^{2} .
\end{aligned}
$$

Damit sind alle Terme der rechten Seite von (4.26) abgeschätzt:

$$
\begin{gathered}
\frac{1}{2} \partial_{t}\left\|\mathbf{e}_{h}^{u}\right\|_{0}^{2}+\frac{3}{4} \nu\left\|\mathbb{D} \mathbf{e}_{h}^{u}\right\|_{0}^{2}+\frac{1}{2} \sum_{K \in \mathcal{T}_{h}}\left(\nu_{K}^{T}\left\|\kappa_{u}\left(\mathbb{D} \mathbf{e}_{h}^{u}\right)\right\|_{0, K}^{2}+\gamma_{K}\left\|\nabla \cdot \mathbf{e}_{h}^{u}\right\|_{0, K}^{2}\right) \\
\leq \frac{3 C_{\mathrm{Korn}}^{2}}{\nu}\left\|\partial_{t} \epsilon^{u}\right\|_{H^{-1}(\Omega)}^{2}+2 \nu\left\|\mathbb{D} \epsilon^{u}\right\|_{0}^{2}+\sum_{K \in \mathcal{T}_{h}} \nu_{K}^{T}\left(\left\|\kappa_{u}\left(\mathbb{D} \epsilon^{u}\right)\right\|_{L^{2}(K)}^{2}+\left\|\kappa_{u}(\mathbb{D} \mathbf{u})\right\|_{L^{2}(K)}^{2}\right) \\
\quad+\sum_{K \in \mathcal{T}_{h}} \min \left(\frac{9 C_{\mathrm{Ko}}^{2}}{\nu}, \frac{1}{\gamma_{K}}\right)\left(\left\|p-\lambda_{h}\right\|_{L^{2}(K)}^{2}+\gamma_{K}^{2}\left\|\nabla \cdot \epsilon^{u}\right\|_{L^{2}(K)}^{2}\right) \\
+\frac{3 C_{\mathrm{LT}}^{2}}{\nu}\left(\left\|\epsilon^{u}\right\|_{0}\left\|\mathbb{D} \epsilon^{u}\right\|_{0}\|\mathbb{D} \mathbf{u}\|_{0}^{2}+\left\|\mathbf{u}_{h}\right\|_{0}\left\|\mathbb{D} \mathbf{u}_{h}\right\|_{0}\left\|\mathbb{D} \epsilon^{u}\right\|_{0}^{2}\right)+\frac{\beta\|\mathbf{g}\|_{\infty}}{3}\left\|\epsilon^{\theta}\right\|_{0}^{2} \\
+\left(\frac{27 C_{\mathrm{LT}}^{4}}{4 \nu^{3}}\|\mathbb{D} \mathbf{u}\|_{0}^{4}+\beta\|\mathbf{g}\|_{\infty}\right)\left(\left\|\mathbf{e}_{h}^{u}\right\|_{0}^{2}+\left\|e_{h}^{\theta}\right\|_{0}^{2}\right) .
\end{gathered}
$$

Jetzt verfahren wir für die Temperatur-Fehlergleichung (4.27) analog und erhalten

$$
\begin{aligned}
\left(\partial_{t} \epsilon^{\theta}, e_{h}^{\theta}\right) & \leq\left\|\partial_{t} \epsilon^{\theta}\right\|_{H^{-1}(\Omega)}\left\|\nabla e_{h}^{\theta}\right\|_{0} \leq \frac{2}{\alpha}\left\|\partial_{t} \epsilon^{\theta}\right\|_{H^{-1}(\Omega)}^{2}+\frac{1}{8} \alpha\left\|\nabla e_{h}^{\theta}\right\|_{0}^{2}, \\
\left(\alpha \nabla \epsilon^{\theta}, \nabla e_{h}^{\theta}\right) & \leq \alpha\left\|\nabla \epsilon^{\theta}\right\|_{0}\left\|\nabla e_{h}^{\theta}\right\|_{0} \leq 2 \alpha\left\|\nabla \epsilon^{\theta}\right\|_{0}^{2}+\frac{1}{8} \alpha\left\|\nabla e_{h}^{\theta}\right\|_{0}^{2} .
\end{aligned}
$$

Auch die Turbulenzmodell-Terme lassen sich ähnlich abschätzen:

$$
\begin{aligned}
\sum_{K \in \mathcal{T}_{h}} \alpha_{K}^{T}\left(\kappa_{\theta}\left(\nabla \epsilon^{\theta}\right), \kappa_{\theta}\left(\nabla e_{h}^{\theta}\right)\right)_{K} & \leq \sum_{K \in \mathcal{T}_{h}} \alpha_{K}^{T}\left\|\kappa_{\theta}\left(\nabla \epsilon^{\theta}\right)\right\|_{L^{2}(K)}^{2}+\sum_{K \in \mathcal{T}_{h}} \frac{\alpha_{K}^{T}}{4}\left\|\kappa_{\theta}\left(\nabla e_{h}^{\theta}\right)\right\|_{L^{2}(K)}^{2}, \\
\sum_{K \in \mathcal{T}_{h}} \alpha_{K}^{T}\left(\kappa_{\theta}(\nabla \theta), \kappa_{\theta}\left(\nabla e_{h}^{\theta}\right)\right)_{K} & \leq \sum_{K \in \mathcal{T}_{h}} \alpha_{K}^{T}\left\|\kappa_{\theta}(\nabla \theta)\right\|_{L^{2}(K)}^{2}+\sum_{K \in \mathcal{T}_{h}} \frac{\alpha_{K}^{T}}{4}\left\|\kappa_{\theta}\left(\nabla e_{h}^{\theta}\right)\right\|_{L^{2}(K)}^{2} .
\end{aligned}
$$

Den konvektiven Term der Temperaturgleichung zerlegen wir wie zuvor bei der Impulsgleichung

$$
c_{s}\left(\mathbf{u}, \theta, e_{h}^{\theta}\right)-c_{s}\left(\mathbf{u}_{h}, \theta_{h}, e_{h}^{\theta}\right)=c_{s}\left(\epsilon^{u}, \theta, e_{h}^{\theta}\right)-c_{s}\left(\mathbf{e}_{h}^{u}, \theta, e_{h}^{\theta}\right)+c_{s}\left(\mathbf{u}_{h}, \epsilon^{\theta}, e_{h}^{\theta}\right)
$$

und verwenden, dass auch der skalare Konvektionsterm durch

$$
c_{s}(\mathbf{u}, \theta, \vartheta) \leq C_{\mathrm{LT}}\|\mathbf{u}\|_{0}^{\frac{1}{2}}\|\mathbb{D} \mathbf{u}\|_{0}^{\frac{1}{2}}\|\nabla \theta\|_{0}\|\nabla \vartheta\|_{0}
$$

abgeschätzt werden kann. Wiederum liefert der mittlere Term, der zwei mal die Diskretisierungsfehler enthält, einen Term, der nicht mit der linken Seite ausgeglichen werden 
kann:

$$
\begin{aligned}
c_{s}\left(\mathbf{e}_{h}^{u}, \theta, e_{h}^{\theta}\right) & \leq C_{\mathrm{LT}}\left\|\mathbf{e}_{h}^{u}\right\|_{0}^{\frac{1}{2}}\left\|\mathbb{D} \mathbf{e}_{h}^{u}\right\|_{0}^{\frac{1}{2}}\|\nabla \theta\|_{0}\left\|\nabla e_{h}^{\theta}\right\|_{0} \\
& \leq \frac{3 C_{\mathrm{LT}}^{4}}{\nu \alpha^{2}}\|\nabla \theta\|_{0}^{4}\left\|\mathbf{e}_{h}^{u}\right\|_{0}^{2}+\frac{1}{4} \nu\left\|\mathbb{D} \mathbf{e}_{h}^{u}\right\|_{0}^{2}+\frac{1}{12} \alpha\left\|\nabla e_{h}^{\theta}\right\|_{0}^{2} .
\end{aligned}
$$

Die anderen beiden Terme lassen sich unproblematisch auf folgende Weise abschätzen

$$
\begin{aligned}
c_{s}\left(\epsilon^{u}, \theta, e_{h}^{\theta}\right) & \leq C_{\mathrm{LT}}\left\|\epsilon^{u}\right\|_{0}^{\frac{1}{2}}\left\|\mathbb{D} \epsilon^{u}\right\|_{0}^{\frac{1}{2}}\|\nabla \theta\|_{0}\left\|\nabla e_{h}^{\theta}\right\|_{0} \\
& \leq \frac{3 C_{\mathrm{LT}}^{2}}{\alpha}\|\nabla \theta\|_{0}^{2}\left\|\epsilon^{u}\right\|_{0}\left\|\mathbb{D} \epsilon^{u}\right\|_{0}+\frac{1}{12} \alpha\left\|\nabla e_{h}^{\theta}\right\|_{0}^{2}
\end{aligned}
$$

und

$$
\begin{aligned}
c_{s}\left(\mathbf{u}_{h}, \epsilon^{\theta}, e_{h}^{\theta}\right) & \leq C_{\mathrm{LT}}\left\|\mathbf{u}_{h}\right\|_{0}^{\frac{1}{2}}\left\|\mathbb{D} \mathbf{u}_{h}\right\|_{0}^{\frac{1}{2}}\left\|\nabla \epsilon^{\theta}\right\|_{0}\left\|\nabla e_{h}^{\theta}\right\|_{0} \\
& \leq \frac{3 C_{\mathrm{LT}}^{2}}{\alpha}\left\|\mathbf{u}_{h}\right\|_{0}\left\|\mathbb{D} \mathbf{u}_{h}\right\|_{0}\left\|\nabla \epsilon^{\theta}\right\|_{0}^{2}+\frac{1}{12} \alpha\left\|\mathbb{D} e_{h}^{\theta}\right\|_{0}^{2} .
\end{aligned}
$$

Die Zusammenfassung aller Abschätzungen für (4.27) liefert

$$
\begin{aligned}
\frac{1}{2} \partial_{t}\left\|e_{h}^{\theta}\right\|_{0}^{2}-\frac{1}{4} \nu\left\|\mathbb{D} \mathbf{e}_{h}^{u}\right\|_{0}^{2}+\frac{1}{2} \alpha\left\|\nabla e_{h}^{\theta}\right\|_{0}^{2}+\frac{1}{2} \sum_{K \in \mathcal{T}_{h}} \alpha_{K}^{T}\left\|\kappa_{\theta}\left(\nabla e_{h}^{\theta}\right)\right\|_{0, K}^{2} \\
\leq \frac{2}{\alpha}\left\|\partial_{t} \epsilon^{\theta}\right\|_{H^{-1}(\Omega)}^{2}+2 \alpha\left\|\nabla \epsilon^{\theta}\right\|_{0}^{2}+\sum_{K \in \mathcal{T}_{h}} \alpha_{K}^{T}\left(\left\|\kappa_{\theta}\left(\nabla \epsilon^{\theta}\right)\right\|_{L^{2}(K)}^{2}+\left\|\kappa_{\theta}(\nabla \theta)\right\|_{L^{2}(K)}^{2}\right) \\
+\frac{3 C_{1}^{2}}{\alpha}\left(\|\nabla \theta\|_{0}^{2}\left\|\epsilon^{u}\right\|_{0}\left\|\mathbb{D} \epsilon^{u}\right\|_{0}+\left\|\mathbf{u}_{h}\right\|_{0}\left\|\mathbb{D} \mathbf{u}_{h}\right\|_{0}\left\|\nabla \epsilon^{\theta}\right\|_{0}^{2}\right)+\frac{3 C_{1}^{4}}{\nu \alpha^{2}}\|\nabla \theta\|_{0}^{4}\left\|\mathbf{e}_{h}^{u}\right\|_{0}^{2}
\end{aligned}
$$

Der negative Term auf der linken Seite wird durch Addieren von (4.29) und (4.30) ausgeglichen, wodurch sich eine Abschätzung der Form (4.12) ergibt. Dabei sind die Funktionen $g_{i}, i=1,2,3$ wie folgt definiert. Bei $g_{1}$ handelt es sich um einen Normähnlichen Term, der den Fehler zusätzlich zur $L^{2}$-Norm kontrolliert:

$$
\begin{aligned}
g_{1}:=\nu\left\|\mathbb{D} \mathbf{e}_{h}^{u}\right\|_{0}^{2} & +\alpha\left\|\nabla e_{h}^{\theta}\right\|_{0}^{2} \\
& +\sum_{K \in \mathcal{T}_{h}}\left(\nu_{K}^{T}\left\|\kappa_{u}\left(\mathbb{D} \mathbf{e}_{h}^{u}\right)\right\|_{0, K}^{2}+\gamma_{K}\left\|\nabla \cdot \mathbf{e}_{h}^{u}\right\|_{0, K}^{2}+\alpha_{K}^{T}\left\|\kappa_{\theta}\left(\nabla e_{h}^{\theta}\right)\right\|_{0, K}^{2}\right) .
\end{aligned}
$$

Nach der Integration über das Intervall $[0, T]$ wird daraus ein Teil von $\left.\left\|\mathbf{e}_{h}^{u}\right\|\left\|_{0, t}^{2}+\right\|\left[e_{h}^{\theta}\right]\right|_{0, t} ^{2}$. In $g_{2}$ sind zum einen Interpolationsfehler und zum anderen Konsistenzfehler zusammengefasst. Diese bestimmen die Konvergenzordnung bezüglich der räumlichen Diskretisie- 
rung:

$$
\begin{gathered}
g_{2}:=\frac{6 C_{\mathrm{Korn}}^{2}}{\nu}\left\|\partial_{t} \epsilon^{u}\right\|_{H^{-1}(\Omega)}^{2}+\frac{4}{\alpha}\left\|\partial_{t} \epsilon^{\theta}\right\|_{H^{-1}(\Omega)}^{2}+4 \nu\left\|\mathbb{D} \epsilon^{u}\right\|_{0}^{2}+4 \alpha\left\|\nabla \epsilon^{\theta}\right\|_{0}^{2} \\
+2 \sum_{K \in \mathcal{T}_{h}}\left(\nu_{K}^{T}\left\|\kappa_{u}\left(\mathbb{D} \epsilon^{u}\right)\right\|_{L^{2}(K)}^{2}+\alpha_{K}^{T}\left\|\kappa_{\theta}\left(\nabla \epsilon^{\theta}\right)\right\|_{L^{2}(K)}^{2}\right) \\
+2 \sum_{K \in \mathcal{T}_{h}}\left(\nu_{K}^{T}\left\|\kappa_{u}(\mathbb{D} \mathbf{u})\right\|_{L^{2}(K)}^{2}+\alpha_{K}^{T}\left\|\kappa_{\theta}(\nabla \theta)\right\|_{L^{2}(K)}^{2}\right) \\
+2 \sum_{K \in \mathcal{T}_{h}} \min \left(\frac{9 C_{\mathrm{Ko}}^{2}}{\nu}, \frac{1}{\gamma_{K}}\right)\left(\left\|p-\lambda_{h}\right\|_{L^{2}(K)}^{2}+\gamma_{K}^{2}\left\|\nabla \cdot \epsilon^{u}\right\|_{L^{2}(K)}^{2}\right) \\
+\frac{6 C_{\mathrm{LT}}^{2}}{\nu}\left(\|\mathbb{D} \mathbf{u}\|_{0}^{2}\left\|\epsilon^{u}\right\|_{0}\left\|\mathbb{D} \epsilon^{u}\right\|_{0}+\left\|\mathbf{u}_{h}\right\|_{0}\left\|\mathbb{D} \mathbf{u}_{h}\right\|_{0}\left\|\mathbb{D} \epsilon^{u}\right\|_{0}^{2}\right)+\frac{2 \beta\|\mathbf{g}\|_{\infty}}{3}\left\|\epsilon^{\theta}\right\|_{0}^{2} \\
+\frac{6 C_{1}^{2}}{\alpha}\left(\|\nabla \theta\|_{0}^{2}\left\|\epsilon^{u}\right\|_{0}\left\|\mathbb{D} \epsilon^{u}\right\|_{0}+\left\|\mathbf{u}_{h}\right\|_{0}\left\|\mathbb{D} \mathbf{u}_{h}\right\|_{0}\left\|\nabla \epsilon^{\theta}\right\|_{0}^{2}\right) .
\end{gathered}
$$

Insbesondere die Konsistenzfehler des Modells in der dritten Zeile sind zu beachten. Diese werden nicht durch die räumliche Diskretisierung beeinflusst, sondern durch die Turbulenzparameter $\nu_{K}^{T}, \alpha_{K}^{T}$ und die Wahl der Fluktuationsoperatoren $\kappa_{u}, \kappa_{\theta}$.

Als letztes bleibt die Funktion $g_{3}$, die in den exponentiellen Term der Gronwallschen Ungleichung eingehen wird:

$$
g_{3}:=\frac{27 C_{\mathrm{LT}}^{4}}{2 \nu^{3}}\|\mathbb{D} \mathbf{u}\|_{0}^{4}+\frac{6 C_{1}^{4}}{\nu \alpha^{2}}\|\nabla \theta\|_{0}^{4}+2 \beta\|\mathbf{g}\|_{\infty} .
$$

Die dritten Potenzen der Viskositäten im Nenner sorgen für eine praktisch geringe Aussagekraft der Fehlerabschätzung, lassen sich jedoch durch eine Analysis der hier gezeigten Art nicht vermeiden.

Um den Beweis des Satzes abzuschließen, müssen noch die Voraussetzungen von Lemma 4.7 geprüft werden. Die Monotonie und Nicht-Negativität sowie die $L^{1}$-Regularität für die meisten Terme sind offenbar erfüllt. Für die Regularität von $g_{3}$ hatten wir entsprechende Regularitätsannahmen an die Lösung gestellt. Lediglich die Terme aus den Abschätzungen der Konvektionsterme müssen gesondert betrachtet werden. Diese werden wir mit Hilfe der a-priori Stabilitätsabschätzungen aus den Lemmata 4.10 und 4.11 sowie den Regularitätsannahmen an $\mathbb{D} \epsilon^{u}$ und $\nabla \epsilon^{\theta}$ abschätzen. Es gilt

$$
\begin{gathered}
\int_{0}^{t}\left\|\mathbf{u}_{h}\right\|\left\|_{0}\right\| \mathbb{D} \mathbf{u}_{h}\left\|_{0}\right\| \mathbb{D} \epsilon^{u}\left\|_{0}^{2} \mathrm{~d} s \leq\right\| \mathbf{u}_{h}\left\|_{L^{\infty}\left(0, t ; L^{2}(\Omega)\right)}\right\| \mathbb{D} \mathbf{u}_{h}\left\|_{L^{2}\left(0, t ; L^{2}(\Omega)\right)}\right\| \mathbb{D} \epsilon^{u} \|_{L^{4}\left(0, t ; L^{2}(\Omega)\right)}^{2}<\infty, \\
\int_{0}^{t}\|\mathbb{D} \mathbf{u}\|_{0}^{2}\left\|\epsilon^{u}\right\|_{0}\left\|\mathbb{D} \epsilon^{u}\right\|_{0} \mathrm{~d} s \leq C_{\mathrm{F}} C_{\mathrm{Korn}}\|\mathbb{D} \mathbf{u}\|_{L^{4}\left(0, t ; L^{2}(\Omega)\right)}^{2}\left\|\mathbb{D} \epsilon^{u}\right\|_{L^{4}\left(0, t ; L^{2}(\Omega)\right)}^{2}<\infty, \\
\int_{0}^{t}\left\|\mathbf{u}_{h}\right\|_{0}\left\|\mathbb{D} \mathbf{u}_{h}\right\|_{0}\left\|\nabla \epsilon^{\theta}\right\|_{0}^{2} \leq\left\|\mathbf{u}_{h}\right\|_{L^{\infty}\left(0, t ; L^{2}(\Omega)\right)}\left\|\mathbb{D} \mathbf{u}_{h}\right\|_{L^{2}\left(0, t ; L^{2}(\Omega)\right)}\left\|\nabla \epsilon^{\theta}\right\|_{L^{4}\left(0, t ; L^{2}(\Omega)\right)}^{2}<\infty, \\
\int_{0}^{t}\|\nabla \theta\|_{0}^{2}\left\|\epsilon^{u}\right\|_{0}\left\|\mathbb{D} \epsilon^{u}\right\|_{0} \leq C_{\mathrm{F}} C_{\mathrm{Korn}}\|\nabla \theta\|_{L^{4}\left(0, t ; L^{2}(\Omega)\right)}^{2}\left\|\mathbb{D} \epsilon^{u}\right\|_{L^{4}\left(0, t ; L^{2}(\Omega)\right)}^{2}<\infty .
\end{gathered}
$$


Als Zwischenresultat erhalten wir so aus dem Gronwallschen Lemma

$$
\begin{aligned}
\left\|\mathbf{e}_{h}^{u}\right\|_{0, t}^{2}+\|\left.\left[e_{h}^{\theta}\right]\right|_{0, t} ^{2} \leq \exp \left(\int_{0}^{t} \frac{27 C_{\mathrm{LT}}^{4}}{2 \nu^{3}}\|\mathbb{D} \mathbf{u}\|_{0}^{4}\right. & \left.+\frac{6 C_{1}^{4}}{\nu \alpha^{2}}\|\nabla \theta\|_{0}^{4}+2 \beta\|\mathbf{g}\|_{\infty} \mathrm{d} s\right) \\
& \cdot\left(\left\|\mathbf{e}_{h}^{u}(0)\right\|_{0}^{2}+\left\|e_{h}^{\theta}(0)\right\|_{0}^{2}+\int_{0}^{t} g_{2}(s) \mathrm{d} s\right),
\end{aligned}
$$

wobei wir $g_{2}$ aus (4.31) nicht noch einmal wiederholt haben. Die Behauptung folgt damit unmittelbar aus

$$
\|\| \mathbf{u}-\mathbf{u}_{h}\left\|_{0, t}^{2}+\left|\left[\theta-\theta_{h}\right]\right|_{0, t}^{2} \leq 2\right\|\left\|\epsilon^{u}\right\|\left\|_{0, t}^{2}+2\left|\left[\epsilon^{\theta}\right]\right|_{0, t}^{2}+2\right\| \mathbf{e}_{h}^{u} \|_{0, t}^{2}+2\left|\left[e_{h}^{\theta}\right]\right|_{0, t}^{2} .
$$

In die Fehlerabschätzung können Interpolationsoperatoren eingesetzt werden, die eine konkrete Approximationsordnung in $h$ liefern. Eine ausführlichere Diskussion der Fehlerabschätzung folgt am Ende dieses Kapitels.

\subsection{Abgeleitete Fehlerabschätzung für den Druck}

Zu der diskreten Lösung $\mathbf{u}_{h}, \theta_{h}$ von (4.1), (4.2) ist aufgrund der inf-sup Bedingung durch (3.33) auch der Druck $p_{h}$ eindeutig bestimmt. Wie wir im vorherigen Abschnitt gesehen haben, hängt die Fehlerabschätzung für die Geschwindigkeit $\mathbf{u}_{h}$ und die Temperatur $\theta_{h}$ aber nicht vom diskreten Druck $p_{h}$, sondern von einer beliebig wählbaren Approximation $\lambda_{h}$ an $p$ ab. Da aber auch der diskrete Druck für die Auswertung von Strömungen eine wichtige Größe ist, sind wir ebenfalls an einer Fehlerabschätzung für $p_{h}$ interessiert.

Satz 4.13 Für den zur diskreten Lösung $\mathbf{u}_{h}, \theta_{h}$ gehörenden Druck $p_{h}$ gilt die Fehlerabschätzung

$$
\begin{gathered}
\left\|p-p_{h}\right\|_{L^{2}\left(0, t ; L^{2}(\Omega)\right)}^{2} \leq 2\left(1+\frac{\sqrt{3}}{b}\right)^{2} \inf _{\tilde{p}_{h} \in Q_{h}}\left\|p-\tilde{p}_{h}\right\|_{L^{2}\left(0, t ; L^{2}(\Omega)\right)}^{2}+C_{u}\left\|\mathbf{u}-\mathbf{u}_{h}\right\|_{0, t}^{2} \\
+\frac{14}{b^{2}}\left\|\partial_{t}\left(\mathbf{u}-\mathbf{u}_{h}\right)\right\|_{L^{2}\left(0, t ; H^{-1}(\Omega)\right)}^{2} \\
+2 \beta^{2} C_{\mathrm{F}}^{2}\|\mathbf{g}\|_{\infty}^{2}\left\|\theta-\theta_{h}\right\|_{L^{2}\left(0, t ; L^{2}(\Omega)\right)}^{2} \\
+\frac{14}{b^{2}} \int_{0}^{t} \sum_{K \in \mathcal{T}_{h}}\left(\nu_{K}^{T}\right)^{2}\left\|\kappa_{u}(\mathbb{D} \mathbf{u})\right\|_{L^{2}(K)}^{2} \mathrm{~d} s
\end{gathered}
$$

für alle $t \in[0, T]$ mit

$$
C_{u}:=\frac{14}{b^{2}} \max _{s \in[0, t]}\left(4 \nu+3 \max _{K \in \mathcal{T}_{h}}\left\{\gamma_{K}, \nu_{K}^{T}\right\}+\frac{C_{\mathrm{LT}}^{2}\left(\left\|\mathbb{D} \mathbf{u}_{h}\right\|_{0}+\|\mathbb{D} \mathbf{u}\|_{0}\right)^{2}}{\nu}\right) .
$$

Beweis: Wir zerlegen $p-p_{h}=\left(p-\tilde{p}_{h}\right)+\left(\tilde{p}_{h}-p_{h}\right)$ in einen Approximations- und einen Diskretisierungsfehler. Diese Zerlegung und die inf-sup Bedingung (2.20) verwenden wir 
für die folgende Abschätzung:

$$
\begin{aligned}
b\left\|p_{h}-p\right\|_{0} & \leq b\left\|p_{h}-\tilde{p}_{h}\right\|_{0}+b\left\|\tilde{p}_{h}-p\right\|_{0} \leq \frac{\left(p_{h}-\tilde{p}_{h}, \nabla \cdot \mathbf{v}_{h}\right)}{\left\|\nabla \mathbf{v}_{h}\right\|_{0}}+b\left\|p-\tilde{p}_{h}\right\|_{0} \\
& \leq \frac{\left(p_{h}-p, \nabla \cdot \mathbf{v}_{h}\right)}{\left\|\nabla \mathbf{v}_{h}\right\|_{0}}+\frac{\left(p-\tilde{p}_{h}, \nabla \cdot \mathbf{v}_{h}\right)}{\left\|\nabla \mathbf{v}_{h}\right\|_{0}}+b\left\|p-\tilde{p}_{h}\right\|_{0} \\
& \leq \frac{\left(p_{h}-p, \nabla \cdot \mathbf{v}_{h}\right)}{\left\|\nabla \mathbf{v}_{h}\right\|_{0}}+(b+\sqrt{3})\left\|p-\tilde{p}_{h}\right\|_{0}
\end{aligned}
$$

Durch Umstellen und Quadrieren der Ungleichung erhalten wir

$$
\left\|p_{h}-p\right\|_{0}^{2} \leq \frac{2\left(p_{h}-p, \nabla \cdot \mathbf{v}_{h}\right)^{2}}{b^{2}\left\|\nabla \mathbf{v}_{h}\right\|_{0}^{2}}+2\left(1+\frac{\sqrt{3}}{b}\right)^{2}\left\|p-\tilde{p}_{h}\right\|_{0}^{2} \quad \forall \tilde{p}_{h} \in Q_{h} .
$$

Um den verbleibenden ersten Term abzuschätzen, bilden wir die Differenz der beiden Variationsgleichungen (2.4) und (3.33) für $\mathbf{u}$ und $\mathbf{u}_{h}$. Dabei verwenden wir zur Abkürzung die Definitionen $\mathbf{e}_{u}:=\mathbf{u}_{h}-\mathbf{u}$ und $e_{\theta}:=\theta_{h}-\theta$ und erhalten

$$
\begin{array}{r}
\left(\partial_{t} \mathbf{e}_{u}, \mathbf{v}_{h}\right)+2 \nu\left(\mathbb{D} \mathbf{e}_{u}, \mathbb{D} \mathbf{v}_{h}\right)+b_{S}\left(\mathbf{u}_{h}, \mathbf{u}_{h}, \mathbf{v}_{h}\right)-b_{S}\left(\mathbf{u}, \mathbf{u}, \mathbf{v}_{h}\right)+\beta\left(\mathbf{g} e_{\theta}, \mathbf{v}_{h}\right) \\
+\sum_{K \in \mathcal{T}_{h}}\left(\gamma_{K}\left(\nabla \cdot \mathbf{e}_{u}, \nabla \cdot \mathbf{v}_{h}\right)_{K}+\nu_{K}^{T}\left(\kappa_{u}\left(\mathbb{D} \mathbf{e}_{u}\right), \mathbb{D} \mathbf{v}_{h}\right)+\nu_{K}^{T}\left(\kappa_{u}(\mathbb{D} \mathbf{u}), \mathbb{D} \mathbf{v}_{h}\right)\right) \\
=\left(p_{h}-p, \nabla \cdot \mathbf{v}_{h}\right) .
\end{array}
$$

Die beiden konvektiven Terme werden zunächst umgestellt und dann erneut die Abschätzung (4.11) aus Lemma 2.2 in Ref. [43] verwendet

$$
\begin{aligned}
& b_{S}\left(\mathbf{u}_{h}, \mathbf{u}_{h}, \mathbf{v}_{h}\right)-b_{S}\left(\mathbf{u}, \mathbf{u}, \mathbf{v}_{h}\right) \\
& \quad=b_{S}\left(\mathbf{u}_{h}, \mathbf{u}_{h}, \mathbf{v}_{h}\right)-b_{S}\left(\mathbf{u}, \mathbf{u}_{h}, \mathbf{v}_{h}\right)+b_{S}\left(\mathbf{u}, \mathbf{u}_{h}, \mathbf{v}_{h}\right)-b_{S}\left(\mathbf{u}, \mathbf{u}, \mathbf{v}_{h}\right) \\
& \quad=b_{S}\left(\mathbf{e}_{u}, \mathbf{u}_{h}, \mathbf{v}_{h}\right)+b_{S}\left(\mathbf{u}, \mathbf{e}_{u}, \mathbf{v}_{h}\right) \leq C_{\mathrm{LT}}\left(\left\|\mathbb{D} \mathbf{u}_{h}\right\|_{0}+\|\mathbb{D} \mathbf{u}\|_{0}\right)\left\|\mathbb{D} \mathbf{e}_{u}\right\|_{0}\left\|\nabla \mathbf{v}_{h}\right\|_{0} .
\end{aligned}
$$


Damit lässt sich folgende Abschätzung bilden

$$
\begin{aligned}
& \frac{\left(p_{h}-p, \nabla \cdot \mathbf{v}_{h}\right)}{\left\|\nabla \mathbf{v}_{h}\right\|_{0}} \leq \frac{1}{\left\|\nabla \mathbf{v}_{h}\right\|_{0}}\left[\left(\partial_{t} \mathbf{e}_{u}, \mathbf{v}_{h}\right)+2 \nu\left(\mathbb{D} \mathbf{e}_{u}, \mathbb{D} \mathbf{v}_{h}\right)+\beta\left(\mathbf{g} e_{\theta}, \mathbf{v}_{h}\right)\right. \\
& +b_{S}\left(\mathbf{u}_{h}, \mathbf{u}_{h}, \mathbf{v}_{h}\right)-b_{S}\left(\mathbf{u}, \mathbf{u}, \mathbf{v}_{h}\right)+\sum_{K \in \mathcal{T}_{h}} \gamma_{K}\left(\nabla \cdot \mathbf{e}_{u}, \nabla \cdot \mathbf{v}_{h}\right)_{K} \\
& \left.+\sum_{K \in \mathcal{T}_{h}} \nu_{K}^{T}\left(\kappa_{u}\left(\mathbb{D} \mathbf{e}_{u}\right), \mathbb{D} \mathbf{v}_{h}\right)_{K}+\sum_{K \in \mathcal{T}_{h}} \nu_{K}^{T}\left(\kappa_{u}(\mathbb{D} \mathbf{u}), \mathbb{D} \mathbf{v}_{h}\right)_{K}\right] \\
& \leq \frac{1}{\left\|\nabla \mathbf{v}_{h}\right\|_{0}}\left[\left\|\partial_{t} \mathbf{e}_{u}\right\|_{H^{-1}(\Omega)}\left\|\nabla \mathbf{v}_{h}\right\|_{0}+2 \nu\left\|\mathbb{D} \mathbf{e}_{u}\right\|_{0}\left\|\mathbb{D} \mathbf{v}_{h}\right\|_{0}\right. \\
& +\beta\|\mathbf{g}\|_{\infty}\left\|e_{\theta}\right\|_{0}\left\|\mathbf{v}_{h}\right\|_{0}+C_{\mathrm{LT}}\left(\left\|\mathbb{D} \mathbf{u}_{h}\right\|_{0}+\|\mathbb{D} \mathbf{u}\|_{0}\right)\left\|\mathbb{D} \mathbf{e}_{u}\right\|_{0}\left\|\nabla \mathbf{v}_{h}\right\|_{0} \\
& +\sqrt{\sum_{K \in \mathcal{T}_{h}}\left(\gamma_{K}\right)^{2}\left\|\nabla \cdot \mathbf{e}_{u}\right\|_{L^{2}(K)}^{2}}\left\|\nabla \cdot \mathbf{v}_{h}\right\|_{0} \\
& +\sqrt{\sum_{K \in \mathcal{T}_{h}}\left(\nu_{K}^{T}\right)^{2}\left\|\kappa_{u}\left(\mathbb{D} \mathbf{e}_{u}\right)\right\|_{L^{2}(K)}^{2}}\left\|\mathbb{D} \mathbf{v}_{h}\right\|_{0} \\
& \left.+\sqrt{\sum_{K \in \mathcal{T}_{h}}\left(\nu_{K}^{T}\right)^{2}\left\|\kappa_{u}(\mathbb{D} \mathbf{u})\right\|_{L^{2}(K)}^{2}}\left\|\mathbb{D} \mathbf{v}_{h}\right\|_{0}\right] \\
& \leq\left\|\partial_{t} \mathbf{e}_{u}\right\|_{H^{-1}(\Omega)}+2 \nu\left\|\mathbb{D} \mathbf{e}_{u}\right\|_{0}+\beta C_{\mathrm{F}}\|\mathbf{g}\|_{\infty}\left\|e_{\theta}\right\|_{0} \\
& +C_{\mathrm{LT}}\left(\left\|\mathbb{D} \mathbf{u}_{h}\right\|_{0}+\|\mathbb{D} \mathbf{u}\|_{0}\right)\left\|\mathbb{D} \mathbf{e}_{u}\right\|_{0}+\sqrt{\sum_{K \in \mathcal{T}_{h}} 3\left(\gamma_{K}\right)^{2}\left\|\nabla \cdot \mathbf{e}_{u}\right\|_{L^{2}(K)}^{2}} \\
& +\sqrt{\sum_{K \in \mathcal{T}_{h}}\left(\nu_{K}^{T}\right)^{2}\left\|\kappa_{u}\left(\mathbb{D} \mathbf{e}_{u}\right)\right\|_{L^{2}(K)}^{2}}+\sqrt{\sum_{K \in \mathcal{T}_{h}}\left(\nu_{K}^{T}\right)^{2}\left\|\kappa_{u}(\mathbb{D} \mathbf{u})\right\|_{L^{2}(K)}^{2}} .
\end{aligned}
$$

Diese können wir quadrieren

$$
\begin{aligned}
\frac{\left(p_{h}-p, \nabla \cdot \mathbf{v}_{h}\right)^{2}}{7\left\|\nabla \mathbf{v}_{h}\right\|_{0}^{2}} \leq\left\|\partial_{t} \mathbf{e}_{u}\right\|_{H^{-1}(\Omega)}^{2}+4 \nu^{2}\left\|\mathbb{D} \mathbf{e}_{u}\right\|_{0}+\beta^{2} C_{\mathrm{F}}^{2}\|\mathbf{g}\|_{\infty}^{2}\left\|e_{\theta}\right\|_{0}^{2} \\
+C_{\mathrm{LT}}^{2}\left(\left\|\mathbb{D} \mathbf{u}_{h}\right\|_{0}+\|\mathbb{D} \mathbf{u}\|_{0}\right)^{2}\left\|\mathbb{D} \mathbf{e}_{u}\right\|_{0}^{2}+\sum_{K \in \mathcal{T}_{h}} 3\left(\gamma_{K}\right)^{2}\left\|\nabla \cdot \mathbf{e}_{u}\right\|_{L^{2}(K)}^{2} \\
+\sum_{K \in \mathcal{T}_{h}}\left(\nu_{K}^{T}\right)^{2}\left\|\kappa_{u}\left(\mathbb{D} \mathbf{e}_{u}\right)\right\|_{L^{2}(K)}^{2}+\sum_{K \in \mathcal{T}_{h}}\left(\nu_{K}^{T}\right)^{2}\left\|\kappa_{u}(\mathbb{D} \mathbf{u})\right\|_{L^{2}(K)}^{2}
\end{aligned}
$$

und in (4.33) einsetzen. Nach Integration über das Intervall $[0, t]$ folgt die Behauptung.

\subsection{Diskussion}

Die beiden Fehlerabschätzungen (4.23) und (4.32) basieren auf besten Approximationen in den Räumen $\mathbf{Z}_{h}, \Theta_{h}$ und $Q_{h}$. Diese können durch das Einsetzen von Interpolationsope- 
ratoren nach oben abgeschätzt werden. Für $\mathbf{Z}_{h}$ muss dabei die Interpolation von $\mathbf{u}$ die diskrete Divergenz-Bedingung erfüllen. Details zu einem entsprechenden Interpolationsoperator wurden in Abschnitt 2.3.3 behandelt. Außerdem treten in den Abschätzungen die vom Turbulenzmodell verursachten Konsistenzfehler

$$
\sum_{K \in \mathcal{T}_{h}} \nu_{K}^{T}\left\|\kappa_{u}(\mathbb{D} \mathbf{u})\right\|_{L^{2}(K)}^{2} \quad \text { und } \quad \sum_{K \in \mathcal{T}_{h}} \alpha_{K}^{T}\left\|\kappa_{\theta}(\nabla \theta)\right\|_{L^{2}(K)}^{2}
$$

auf. Da die Fluktuationsoperatoren $\kappa_{u / \theta}$ durch Projektionen auf Grobräume definiert sind, spielt hier die Interpolationseigenschaft der Grobräume eine wichtige Rolle. Handelt es sich bei diesen um Ansatzräume mit niedriger polynomieller Ordnung, müssen die turbulenten Viskositätsparameter $\nu_{K}^{T}$ und $\alpha_{K}^{T}$ proportional zu einer entsprechenden Potenz der Gitterweite $h$ sein.

Korollar 4.14 Für den Fall $k \geq 2$ und

$$
\begin{gathered}
\mathbf{V}_{h} \times Q_{h} \times \Theta_{h}=\left[\mathcal{Q}_{k, h}\right]^{d} \times \mathcal{Q}_{k-1, h} \times \mathcal{Q}_{k, h}, \\
\mathbf{L}_{h}=\left[\mathcal{Q}_{k-2, h}^{\text {disc }}\right]^{d \times d}, \quad \mathbf{M}_{h}=\left[\mathcal{Q}_{k-2, h}^{\text {disc }}\right]^{d}, \quad \nu_{K}^{T}, \alpha_{K}^{T} \sim h^{2}
\end{gathered}
$$

erhalten wir unter der Annahme $(\mathbf{u}, p, \theta)(t) \in\left[W^{k+1,2}(\Omega)\right]^{d} \times W^{k, 2}(\Omega) \times W^{k+1,2}(\Omega)$ für alle $t \in(0, T]$ die Fehlerordnung $\mathcal{O}\left(h^{k}\right)$.

Der exponentielle Term in (4.23) beinhaltet keine Diskretisierungsfehler, sondern ausschließlich die Parameter und die Lösung des kontinuierlichen Problems. Ein exponentielles Anwachsen des Fehlers kann jedoch nur für einen beschränkten Zeitraum zu Beginn der Rechnung gelten, da zusätzlich schärfere Stabilitätsabschätzungen bewiesen wurden (siehe Lemmata 4.10 und 4.11), über die der Fehler mittels der Dreiecksungleichung ebenfalls abgeschätzt werden kann. 


\section{Kapitel 5}

\section{Die zeitliche Diskretisierung, Linearisierung und Entkopplung}

Im vorherigen Kapitel wurde das Oberbeck-Boussinesq-Modell durch ein FiniteElemente-Verfahren räumlich diskretisiert. Das resultierende Anfangswertproblem mit algebraischen Nebenbedingungen erfordert spezielle Sorgfalt bei der zeitlichen Diskretisierung, da sonst Probleme bei der Einhaltung der Nebenbedingungen und der Approximation der algebraischen Variablen auftreten.

Ziel dieses Abschnittes ist es, eine Auswahl bekannter Verfahren mit möglichst guten theoretischen Eigenschaften zu treffen und modular zu implementieren, um diese dann numerisch genauer zu untersuchen. Insbesondere sollen Verfahren verschiedener Familien mit unterschiedlicher algorithmischer Komplexität verglichen werden. Ein wichtiger Punkt wird die Behandlung der Nichtlinearität im Gleichungsmodell sein.

Um nicht durch die Notation des Oberbeck-Boussinesq-Modells abgelenkt zu werden, werden wir in diesem Abschnitt die in der Literatur gängige Notation für gewöhnliche Differentialgleichungen verwenden. Dabei bezeichnen die Vektoren $y=y(t) \in \mathbb{R}^{k}$, $z=z(t) \in \mathbb{R}^{l}$ die Lösung des Anfangswertproblems und $t$ weiterhin die Zeit. Bezogen auf die Finite-Elemente-Diskretisierung des Oberbeck-Boussinesq-Modells handelt es sich meist bei $y$ um die Koeffizienten der Basisfunktionen für das Geschwindigkeitsfeld und die Temperatur, während $z$ die Koeffizienten der Basisfunktionen für den Druck enthält. Wir haben also die Funktionenräume verlassen und arbeiten im reellen Standardvektorraum. 


\subsection{Differentiell algebraische Gleichungen}

Differentiell algebraische Gleichungen (differential algebraic equation, DAE) sind allgemein Gleichungen der Form

$$
F\left(t, y, y^{\prime}\right)=0,
$$

wobei $\partial F / \partial y^{\prime}$ singulär sein kann. Da DAEs in dieser allgemeinen Form schwierig analytisch zu untersuchen sind, werden wir die Betrachtung auf für unsere Zwecke ausreichende semi-explizite Gleichungen einschränken.

Definition 5.1 Eine differentiell algebraische Gleichung in semi-expliziter Form ist durch

$$
\begin{aligned}
y^{\prime} & =f(t, y, z), \\
0 & =g(t, y, z)
\end{aligned}
$$

und Anfangsbedingungen $y(0)=y_{0}, z(0)=z_{0}$ gegeben. Die Anfangswerte $y_{0}, z_{0}$ nennen wir konsistent, falls $0=g\left(0, y_{0}, z_{0}\right)$ gilt.

Differentiell algebraische Gleichungen entstehen häufig als Grenzproblem eines singulär gestörten Problems (singular perturbation problem, SPP), bei dem ein kleiner Parameter $\varepsilon$ gegen Null geht:

$$
\begin{aligned}
y^{\prime} & =f(t, y, z), \\
\varepsilon z^{\prime} & =g(t, y, z) .
\end{aligned}
$$

Diese Sichtweise trifft physikalisch nicht auf unser Problem zu, kann aber zur Konstruktion von Verfahren genutzt werden, siehe dazu die künstliche Kompressibilitäts Methode (Artificial Compressibility Method) in Ref. [13].

Zur numerischen Behandlung des Oberbeck-Boussinesq-Modells werden wir auf zwei spezielle Typen von differentiell algebraischen Gleichungen eingehen.

\section{Hessenberg Index-1 DAE}

Zusätzlich zu der Standardform für semi-explizite DAE (5.1) gelte folgende Annahme:

$$
g_{z}(t, y, z) \quad \text { ist invertierbar in einer Umgebung der Lösung. }
$$

Dann handelt es sich bei (5.1) um eine semi-explizite Hessenberg Index-1 DAE.

Zur Überprüfung des Index können wir die zweite Gleichung nach der Zeit differenzieren und erhalten $0=g_{t}+g_{y} \circ y^{\prime}+g_{z} \circ z^{\prime}$, was sich unter der Annahme (5.3) zu

$$
z^{\prime}=-g_{z}^{-1} \circ\left(g_{t}+g_{y} \circ y^{\prime}\right)
$$


umstellen lässt. Wir erhalten also durch einfache Differentiation eine explizite gewöhnliche Differentialgleichung, es handelt sich demnach um eine differentiell algebraische Gleichung mit dem Index 1.

Mit der Annahme (5.3) und dem Satz über implizite Funktionen lässt sich $z=G(y)$ lokal eindeutig bestimmen und einsetzen. So lässt sich die differentiell algebraische Gleichung als gewöhnliche Differentialgleichung im Zustandsraum

$$
y^{\prime}=f(t, y, G(y))
$$

formulieren. Darauf können beliebige Zeitdiskretisierungsverfahren angewendet werden. Dieser indirekte Zugang ist für numerische Verfahren nicht immer empfehlenswert, da z.B. Probleme auftreten können, wenn $z=G(y)$ nicht hinreichend genau gelöst werden kann bzw. aufwendig zu berechnen ist.

Exemplarisch betrachten wir an dieser Stelle den Fall von Dirichlet-Randbedingungen. Dazu bezeichnen wir die auf dem Dirichlet-Rand liegenden Freiheitsgrade mit $z$ und die vorgegebenen Randwerte mit $z_{b}$. Die Randbedingung lautet dann

$$
0=z(t)-z_{b}(t)=: g(t, z)=: g(t, y, z) \text {. }
$$

Es ist also $g_{z}=I d_{z}, g_{y}=0$ und folglich vereinfacht sich (5.4) zu $z^{\prime}=z_{b}^{\prime}$. In diesem einfachen Fall ist $z=G(y)=z_{b}$, was keinen zusätzlichen Aufwand zur Bestimmung von $G$ erforderlich macht. Wir werden daher Zugang (5.5) für die Einarbeitung von Randwerten verwenden und Variablen, die zu Freiheitsgraden auf dem entsprechenden Rand gehören, nicht weiter in der Notation berücksichtigen.

Bei der Implementierung von Finite-Elemente-Software bleiben Randwerte häufig als Komponenten der Vektoren von Freiheitsgraden erhalten. Es sei daher darauf hingewiesen, dass bei jeder Auswertung von $f$ korrekte Randwerte verwendet werden müssen und z.B. zeitliche Extrapolationsformeln für diese Freiheitsgrade nicht verwendet werden dürfen, ohne mögliche Ordnungsreduktionseffekte zu berücksichtigen.

\section{Hessenberg Index-2 DAE}

Definition 5.2 Wir fordern jetzt, dass die Nebenbedingung unabhängig von den algebraischen Variablen ist, die DAE also von der Form

$$
\begin{aligned}
y^{\prime} & =f(t, y, z), \\
0 & =g(t, y)
\end{aligned}
$$

ist. Zusätzlich nehmen wir an:

$$
g_{y}(y) \circ f_{z}(t, y, z) \quad \text { ist invertierbar in einer Umgebung der Lösung. }
$$


Dann handelt es sich bei (5.7) um eine semi-explizite Hessenberg Index-2 DAE. Wieder differenzieren wir die zweite Gleichung, um den Index zu bestimmen:

$$
0=g_{y}(t, y) \circ y^{\prime}+g_{t}=g_{y}(t, y) \circ f(t, y, z)+g_{t}(t, y) \text {. }
$$

Diesmal ergibt sich im ersten Schritt noch keine Differentialgleichung für $z$. Durch erneutes Differenzieren erhalten wir aber unter anderem den Term $g_{y}(t, y) \circ f_{z}(t, y, z) \circ z^{\prime}$, den wir unter der Annahme (5.8) durch Multiplikation mit $\left(g_{y} \circ f_{z}\right)^{-1}$ nach $z^{\prime}$ umstellen können, um eine explizite gewöhnliche Differentialgleichung zu erhalten. Die differentiell algebraische Gleichung hat also den Index 2.

Übertragen wir die Notation dieses Kapitels auf das Blocksystem (2.21), so erhalten wir $g_{y}=B^{\top}=\operatorname{div}_{h}$ und $f_{z}=M_{u}^{-1} B=\operatorname{grad}_{h}$, die diskreten Divergenz- und Gradientenoperatoron. Das Produkt $\operatorname{div}_{h} \circ \operatorname{grad}_{h}=B^{T} M_{u}^{-1} B=\Delta_{h}$ entspricht einer gemischten Diskretisierung des Laplace-Operators $\Delta_{h}: Q_{h} \rightarrow Q_{h} *$. Wegen der inf-sup Bedingung (2.20) ist $\Delta_{h}$ invertierbar und die inverse Abbildung beschränkt. Für das OberbeckBoussinesq-Modell handelt es sich beim Druck $p$ also um eine algebraische Variable mit dem Index 2.

\subsection{Grundlagen der zeitlichen Diskretisierung}

Gegeben sei ein Zeitgitter: $t_{0}<t_{1}<\cdots<t_{N}=T$ mit $\tau_{n}=t_{n+1}-t_{n}$. Dieses kann z.B. als äquidistante Zerlegung des Intervalls vorgegeben sein oder während der Rechnung durch eine an einen Fehlerschätzer gekoppelte adaptive Schrittweitensteuerung erzeugt werden. Gesucht ist eine diskrete Lösung $y_{n} \approx y\left(t_{n}\right)$ für $n=1, \ldots, N$.

Bei Zeitschrittverfahren wird zwischen Ein- und Mehrschrittverfahren unterschieden. Einschrittverfahren verwenden zur Berechnung von $y_{n+1}$ ausschließlich $y_{n}$, während ein $k$-Schrittverfahren die letzten $k$ bekannten Werte $y_{n}, \ldots, y_{n-k+1}$ verwendet. Weiterhin wird zwischen ein- und mehrstufigen Verfahren unterschieden. Ein mehrstufiges Verfahren berechnet mehrere Zwischenwerte sequentiell oder durch ein gekoppeltes Gleichungssystem, während ein einstufiges Verfahren $y_{n+1}$ direkt aus den gegebenen Daten berechnet.

Für die Definitionen von Konsistenz- und Konvergenzordnung sowie der im Folgenden verwendeten Stabilitätsbegriffe und für weitere allgemeine Grundlagen, die hier nicht alle wiederholt werden sollen, verweisen wir auf die umfangreiche Literatur zu diesen Themen $[3,30,31]$.

\subsection{BDF- und sBDF-Verfahren}

Die Backward Differentiation Formula-Verfahren (BDF-Verfahren) gehören zur Klasse der impliziten, linearen Mehrschrittverfahren. Es handelt sich um Kollokationsverfah- 
ren, zu deren Konstruktion Interpolationspolynome $\hat{y}, \hat{z}$ vom Grad $k$ durch die letzten $k$ Werte $\left(t_{n}, y_{n}, z_{n}\right), \ldots,\left(t_{n-k+1}, y_{n-k+1}, z_{n-k+1}\right)$ gelegt werden und gefordert wird, dass die Differentialgleichung (5.1) für die Interpolationspolynome $\hat{y}, \hat{z}$ zum Zeitpunkt $t_{n+1}$ erfüllt wird. Die algebraischen Nebenbedingungen werden also nur zum Zeitpunkt $t_{n+1}$ gefordert. Die Ableitung des Interpolationspolynoms entspricht einem RückwärtsDifferenzenquotienten, der den Verfahren ihren Namen gibt. Für konstante Zeitschrittweite $\tau_{n}=\tau$ sind die Verfahren durch

$$
\hat{y}^{\prime}\left(t_{n+1}\right)=\frac{1}{\tau} \sum_{i=1}^{k} \alpha_{i} y_{n+1-i}=f\left(t_{n+1}, y_{n+1}, z_{n+1}\right), \quad 0=g\left(y_{n+1}\right)
$$

gegeben. Die Koeffizienten $\alpha_{i}$ für $k=1, \ldots, 6$ sowie der Winkel der $A(\alpha)$-Stabilität sind in Tabelle 5.1 gegeben. Für $k>6$ sind die Verfahren nicht stabil. A-Stabilität ist nur für Verfahren bis zur Ordnung 2 erfüllt. Für $k=1$ erkennen wir das klassische implizite Euler-Verfahren.

Eine spezielle Eigenschaft der BDF-Verfahren ist, dass $f(t, y, z)$ nur am Ende des aktuellen Zeitschritts ausgewertet wird, was ihnen sehr gute Stabilitätseigenschaften verleiht. Ferner gehen die algebraischen Variablen aus vorherigen Zeitschritten nicht in die Rechnung mit ein, was die Initialisierung vereinfacht und ebenfalls zu den günstigen Eigenschaften der Verfahren beiträgt, da es keine Fortpflanzungsfehler für die algebraischen Variablen gibt. Die BDF-Verfahren leiden nicht unter Ordnungsreduktion für steife Probleme bzw. DAE Probleme mit Index 1 oder 2. Die volle Ordnung $p=k$ bleibt auch für alle Variablen mit Index 1 und Index 2 erhalten (siehe Theoreme VI.1.2 und VI.6.5, [31]).

Um ein Mehrschrittverfahren zu starten, werden mehrere Startwerte benötigt, die bestimmte Fehlerordnungen erfüllen müssen. D.h. es reicht normalerweise nicht aus, die Startwerte mit Verfahren niedrigerer Ordnung aus der gleichen Familie zu starten. Häufig benötigen Simulationen physikalischer Systeme aber ohnehin eine Einlaufphase, um einen gewissen statistischen Gleichgewichtszustand zu erreichen, bevor mit Messungen oder der Auswertung begonnen wird. In diesem Fall spielt es keine Rolle, wie die Mehrschrittverfahren gestartet werden.

Eine weitere Schwierigkeit entsteht durch variable Zeitschrittweiten. In [3, Abschnitt 5.5.1] werden dazu verschiedene Ansätze beschrieben. Im Wesentlichen wird zwischen Formeln mit variablen und Formeln mit konstanten Koeffizienten unterschieden. Bei Formeln mit variablen Koeffizienten werden die Gewichte für die Differenzenquotienten aus den Ableitungen eines Interpolationspolynoms mit nicht äquidistanten Stützstellen für jeden Zeitschritt neu berechnet. Dies führt zu guten Stabilitätseigenschaften, kann aber eine effiziente Implementierung erschweren oder verhindern. Die zweite Möglichkeit besteht darin, das Kollokationspolynom des letzten Zeitschritts an für den nächsten Schritt passenden äquidistanten Stützstellen auszuwerten und immer mit den Koeffizienten für konstantes Schrittweitenverhältnis zu rechnen. Dadurch entstehen jedoch zusätzlich Interpolationsfehler und insbesondere bei häufiger oder großer Änderung der Zeitschrittweite können Stabilitätsprobleme auftreten. 


\begin{tabular}{|c|c|c|c|c|c|c|c|c|c|c|c|c|c|c|}
\hline \multirow[b]{2}{*}{$p=k$} & \multirow[b]{2}{*}{$A(\alpha)$} & \multicolumn{7}{|c|}{ Rückwärts Differenzenquotient } & \multicolumn{6}{|c|}{ Extrap.-Formel (5.11) } \\
\hline & & $\alpha_{0}$ & $\alpha_{1}$ & $\alpha_{2}$ & $\alpha_{3}$ & $\alpha_{4}$ & $\alpha_{5}$ & $\alpha_{6}$ & $\gamma_{1}$ & $\gamma_{2}$ & $\gamma_{3}$ & $\gamma_{4}$ & $\gamma_{5}$ & $\gamma_{6}$ \\
\hline 1 & $90.00^{\circ}$ & 1 & -1 & & & & & & 1 & & & & & \\
\hline 2 & $90.00^{\circ}$ & $\frac{3}{2}$ & $-\frac{4}{2}$ & $\frac{1}{2}$ & & & & & 2 & -1 & & & & \\
\hline 3 & $86.03^{\circ}$ & $\frac{11}{6}$ & $-\frac{18}{6}$ & $\frac{9}{6}$ & $-\frac{2}{6}$ & & & & 3 & -3 & 1 & & & \\
\hline 4 & $73.35^{\circ}$ & $\frac{25}{12}$ & $-\frac{48}{12}$ & $\frac{36}{12}$ & $-\frac{16}{12}$ & $\frac{3}{12}$ & & & 4 & -6 & 4 & -1 & & \\
\hline 5 & $51.84^{\circ}$ & $\frac{137}{60}$ & $-\frac{300}{60}$ & $\frac{300}{60}$ & $-\frac{200}{60}$ & $\frac{75}{60}$ & $-\frac{12}{60}$ & & 5 & -10 & 10 & -5 & 1 & \\
\hline 6 & $17.84^{\circ}$ & $\frac{147}{60}$ & $-\frac{360}{60}$ & $\frac{450}{60}$ & $-\frac{400}{60}$ & $\frac{225}{60}$ & $-\frac{72}{60}$ & $\frac{10}{60}$ & 6 & -15 & 20 & -15 & 6 & -1 \\
\hline
\end{tabular}

Tabelle 5.1: Eigenschaften und Koeffizienten der BDF Verfahren.

Pro Zeitschritt ist unabhängig von der Ordnung nur ein nichtlineares Problem zu lösen, was durch einfache Fixpunkt- oder Newtoniteration erfolgt. Die Konvergenzgeschwindigkeit hängt von der Zeitschrittweite ab, für kleine Zeitschrittweite wird der Einfluss der Nicht-Linearität gegenüber dem linearen Term des Differenzenquotienten schwächer wodurch die Konvergenz beschleunigt wird. Außerdem stehen in der Regel bei kleineren Zeitschrittweiten bessere Startwerte für die Iterationsverfahren zur Verfügung. Diese Abhängigkeit der Iterationszahl von der Zeitschrittweite wird für die Fixpunktiteration in Abschnitt 6.5 numerisch genauer untersucht.

Zur Vermeidung eines Iterationsverfahrens für die nichtlinearen Probleme können nichtlineare Terme explizit in der Zeit extrapoliert werden. In [5] finden sich dazu unter anderem die SBDF-Verfahren, die auf den BDF-Verfahren basieren, den Konvektionsterm aber vollständig explizit behandeln. Dazu wird die Impulsgleichung additiv in einen linearen Term $f_{\text {lin }}$ und einen nichtlinearen Term $f_{\neg \text { lin }}$ zerlegt:

$$
y^{\prime}=f(t, y, z)=f_{\operatorname{lin}}(t, y, z)+f_{\neg \operatorname{lin}}(y) .
$$

Dabei nehmen wir an, dass nur die differentielle Variable $y$ nichtlinear in der Gleichung auftritt, wie es beim Oberbeck-Boussinesq-Modell der Fall ist. Die SBDF-Verfahren sind dann gegeben durch

$$
\frac{1}{\tau} \sum_{i=0}^{k} \alpha_{i} y_{n+1-i}=f_{\text {lin }}\left(t_{n+1}, y_{n+1}, z_{n+1}\right)+\sum_{i=1}^{k} \gamma_{i} f_{\neg \operatorname{lin}}\left(y_{n+1-i}\right), \quad 0=g\left(y_{n+1}\right) .
$$

Der nichtlineare Anteil wird vollständig explizit extrapoliert. Die entsprechenden Koeffizienten $\gamma_{i}$ sind in Tabelle 5.1 angegeben. Angewendet auf das Oberbeck-BoussinesqModell müssen dann in jedem Zeitschritt nur noch ein lineares Stokes-Problem und ein Poisson-Problem (beide mit zusätzlichem Reaktionsterm, aber ohne Konvektionsterme) gelöst werden, die nicht mehr miteinander gekoppelt sind. Die SBDF-Verfahren haben jedoch schlechte Stabilitätseigenschaften bei konvektionsdominanten Problemen. Da aber 
ohnehin ein lineares Problem gelöst werden muss, können wir auch eine andere Form der Linearisierung wählen:

$$
f(t, y, z)=f_{\text {semi-lin }}(t, w=y, y, z) .
$$

Für das zweite Argument $w$ werden wir extrapolierte Werte einsetzen, während das dritte Argument $y$ nur linear in der Gleichung auftritt und implizit behandelt wird. Diese Variante semi-impliziter BDF-Verfahren werden wir sBDF-Verfahren nennen. Sie ist gegeben durch:

$$
\begin{aligned}
\frac{1}{\tau} \sum_{i=0}^{k} \alpha_{i} y_{n+1-i} & =f_{\text {semi-lin }}\left(t_{n+1}, y_{n+1}^{*}, y_{n+1}, z_{n+1}\right), \quad 0=g\left(y_{n+1}\right) \\
y_{n+1}^{*} & =\sum_{i=1}^{k} \gamma_{i} y_{n+1-i} .
\end{aligned}
$$

Für das Oberbeck-Boussinesq-Modell können wir die Konvektionsterme so linearisieren, dass $f\left(t_{n+1}, y_{n+1}^{*} ; \cdot, z_{n+1}\right)$ günstige Eigenschaften hat. Insbesondere haben bei einer schiefsymmetrischen Diskretisierung alle Eigenwerte negativen Realteil, wodurch die Stabilität der sBDF-Verfahren nicht negativ beeinflusst wird.

Bei impliziten BDF-Verfahren können die extrapolierten Werte $y_{n+1}^{*}$ auch als Startwert der Fixpunktiteration verwendet werden, da es sich dabei in der Regel um eine bessere Approximation an $y_{n+1}$ handelt, als z.B. bei $y_{n}$.

\subsection{Implizite Runge-Kutta-Verfahren}

Ein $s$-stufiges Runge-Kutta-Verfahren (RKV) mit Koeffizienten $a_{i j}, b_{j}, c_{i}, i, j=1, \ldots, s$ ist gegeben durch:

$$
\begin{array}{rlrl}
Y_{i n} & =y_{n}+\tau_{n} \sum_{j=1}^{s} a_{i j} f\left(t_{n}+c_{j} \tau_{n}, Y_{j n}, Z_{j n}\right) & & \text { für } i=1, \ldots, s, \\
0 & =g\left(Y_{i n}\right) & & \text { für } i=1, \ldots, s, \\
y_{n+1} & =y_{n}+\tau_{n} \sum_{j=1}^{s} b_{j} f\left(t_{n}+c_{j} \tau_{n}, Y_{j n}, Z_{j n}\right) . &
\end{array}
$$

Man nennt ein RKV explizit (ERK), falls $a_{i j}=0$ für $j \geq i$. In diesem Fall lässt sich $Y_{k n}$ explizit aus den bereits bekannten Werten $y_{n}, Y_{1 n}, \ldots, Y_{(k-1) n}, Z_{1 n}, \ldots, Z_{(k-1) n}$ gemäß Gleichung (5.12a) berechnen. In der Regel erfüllt $Y_{k n}$ dann aber die Bedingung (5.12b) nicht. Ohne weitere Anpassungen ist das System (5.12) für ERK somit nicht wohlgestellt.

Ist ein RKV nicht explizit, nennt man es implizit (IRK). Da wir ohnehin an den vorteilhaften Eigenschaften impliziter Verfahren interessiert sind, werden wir nicht weiter auf 
die Anwendung expliziter RKV auf DAE-Probleme eingehen. Für implizite RKV muss ein gekoppeltes System nichtlinearer Gleichungen gelöst werden. Ein Spezialfall sind diagonal implizite Runge-Kutta-Verfahren (DIRK) mit $a_{i i} \neq 0$ und $a_{i j}=0$ für $j>i$. Für diese muss nur ein gestaffeltes System nichtlinearer Gleichungen gelöst werden, d.h. es können sequentiell die Stufen $Y_{k n}$ für $k=1, \ldots, s$ nacheinander bestimmt werden.

Ein RKV heißt steif-stabil, falls $a_{s j}=b_{j}, j=1, \ldots, s$. In diesem Fall gilt $y_{n+1}=Y_{s n}$ und somit auch $0=g\left(y_{n+1}\right)$, was ansonsten nicht explizit durch das Verfahren gefordert wird. Wegen dieser für DAE-Probleme wichtigen Eigenschaft, aber auch wegen guter Stabilitätseigenschaften werden wir uns auf die Betrachtung von steif-stabilen Verfahren beschränken.

\subsection{1 Äquivalente Formulierung}

Für die folgende Umformung werden wir annehmen, dass die Koeffizientenmatrix $A$ des IRK-Verfahrens invertierbar ist. Bei DIRK-Verfahren ist dies immer der Fall.

Die Berechnung der Stufen des DIRK-Verfahrens kann geschrieben werden als:

$$
\left(\begin{array}{c}
Y_{1 n} \\
\vdots \\
Y_{s n}
\end{array}\right)=\left(\begin{array}{c}
y_{n} \\
\vdots \\
y_{n}
\end{array}\right)+\tau_{n} A\left(\begin{array}{c}
f\left(t_{n}+c_{1} \tau_{n}, Y_{1 n}, Z_{1 n}\right) \\
\vdots \\
f\left(t_{n}+c_{s} \tau_{n}, Y_{s n}, Z_{s n}\right)
\end{array}\right), \quad 0=\left(\begin{array}{c}
g\left(Y_{1 n}\right) \\
\vdots \\
g\left(Y_{s n}\right)
\end{array}\right) \text {. }
$$

Durch Hinzufügen von $y_{n}$ zum Stufenvektor $\left(Y_{1 n}, \ldots, Y_{s n}\right)^{\top}$ erhalten wir:

$$
\underbrace{\left(\begin{array}{ccccc}
-1 & 1 & 0 & \cdots & 0 \\
-1 & 0 & 1 & \cdots & 0 \\
\vdots & \vdots & \vdots & \ddots & \vdots \\
-1 & 0 & 0 & \cdots & 1
\end{array}\right)}_{=: D_{s}}\left(\begin{array}{c}
y_{n} \\
Y_{1 n} \\
\vdots \\
Y_{s n}
\end{array}\right)=\tau_{n} A\left(\begin{array}{c}
f\left(t_{n}+c_{1} \tau_{n}, Y_{1 n}, Z_{1 n}\right) \\
\vdots \\
f\left(t_{n}+c_{s} \tau_{n}, Y_{s n}, Z_{s n}\right)
\end{array}\right), \quad 0=\left(\begin{array}{c}
g\left(Y_{1 n}\right) \\
\vdots \\
g\left(Y_{s n}\right)
\end{array}\right) \text {. }
$$

Ist die Koeffizientenmatrix $A$ eines IRK-Verfahrens invertierbar, so gilt:

$$
\frac{1}{\tau_{n}}\left[A^{-1} D_{s}\right]\left(\begin{array}{c}
y_{n} \\
Y_{1 n} \\
\vdots \\
Y_{s n}
\end{array}\right)=\left(\begin{array}{c}
f\left(t_{n}+c_{1} \tau_{n}, Y_{1 n}, Z_{1 n}\right) \\
\vdots \\
f\left(t_{n}+c_{s} \tau_{n}, Y_{s n}, Z_{s n}\right)
\end{array}\right), \quad 0=\left(\begin{array}{c}
g\left(Y_{1 n}\right) \\
\vdots \\
g\left(Y_{s n}\right)
\end{array}\right) .
$$

In dieser Darstellung tritt in jeder Gleichung $f$ nur noch einmal auf und die Zeilen von $Q=\frac{1}{\tau_{n}} A^{-1} D_{s}$ lassen sich als diskrete Differenzenquotienten für die Zwischenstellen $t_{n}+c_{i} \tau_{n}$ deuten. Es sind keine weiteren expliziten Terme auszuwerten. Die einzelnen Gleichungen haben damit genau die gleiche Form wie ein Schritt der im vorherigen Abschnitt vorgestellten BDF-Verfahren.

Da es sich bei RKV um Einschrittverfahren handelt, kann im Gegensatz zu den BDFVerfahren die Zeitschrittweite beliebig von einem Schritt zum nächsten variiert werden. Die Stabilitätseigenschaften ändern sich dadurch nicht. 


\subsubsection{Steif-stabile DIRK-Verfahren}

DIRK-Verfahren haben den Vorteil, dass kein gekoppeltes System nichtlinearer Gleichungen gelöst werden muss. Es muss lediglich für jede Stufe eine nichtlineare Gleichung gelöst werden. Die günstigsten Eigenschaften haben steif-stabile Verfahren, die für die Ordnungen $p=s \leq 3$ eindeutig durch die Ordnungbedingungen bestimmt sind [31].

Für $s=1$ handelt es sich wieder um das implizite Euler-Verfahren, das diesmal durch das folgende Butcher-Schema gegeben ist:

$$
\begin{array}{l|l}
c & A \\
\hline & b
\end{array} \widehat{=} \quad \begin{array}{l|l}
1 & 1 \\
\hline & 1
\end{array} .
$$

Das zweistufiges Verfahren zweiter Ordnung ist durch

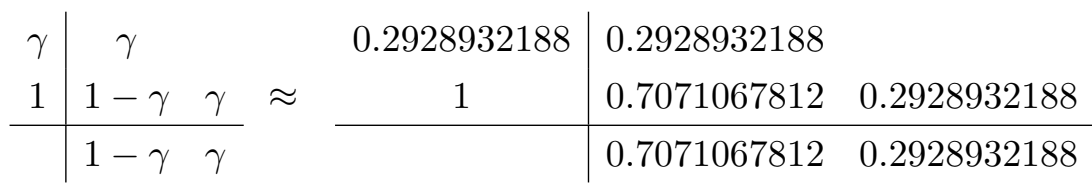

mit $\gamma=\frac{2-\sqrt{2}}{2}$ gegeben. Es folgt das Schema für das dreistufiges Verfahren dritter Ordnung:

\begin{tabular}{c|ccc|crrrr}
$\gamma$ & $\gamma$ & & & 0.4358665215 & 0.4358665215 & & & \\
0.7179332608 & 0.2820667392 & 0.4358665215 & \\
$\frac{1+\gamma}{2}$ & $\frac{1-\gamma}{2}$ & $\gamma$ & & $\approx$ & 1 & 1.2084966490 & -0.6443631710 & 0.4358665215 \\
1 & $b_{1}$ & $b_{2}$ & $\gamma$
\end{tabular}$\quad \begin{array}{cccccc}0.2084966490 & -0.6443631710 & 0.4358665215\end{array}$

mit der mittleren Wurzel $\gamma$ von $6 x^{3}-18 x^{2}+9 x-1=0$ und $b_{1}=-\frac{3}{2} \gamma^{2}+4 \gamma-\frac{1}{4}$, $b_{2}=\frac{3}{2} \gamma^{2}-5 \gamma+\frac{5}{4}$.

Ein vierstufiges steif-stabiles DIRK-Verfahren vierter Ordnung existiert nicht. Ein vierstufiges L-stabiles Verfahren vierter Ordnung mit interessanten Eigenschaften ist durch das folgende Butcher-Schema gegeben:

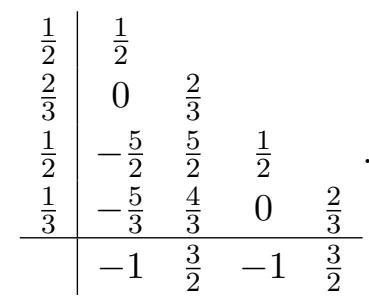


Die besondere Struktur erlaubt es, die zweite und vierte Stufe jeweils parallel zur ersten und dritten Stufe zu berechnen, da diese nicht voneinander abhängen. Eine Implementierung lässt sich somit an dieser Stelle mit perfekter Skalierung in zwei unabhängige Teilprobleme zerlegen. Dieses Beispiel zeigt, dass schon bei der Wahl der Verfahren Aspekte wie die Parallelisierung berücksichtigt werden können, was jedoch zu einer starken Abhängigkeit verschiedener Programmteile führt. In der Praxis werden Verfahren bevorzugt, die sich modular implementieren und leicht austauschen lassen. Ein direkter Einfluss der Zeitdiskretisierung auf Aspekte der Parallelisierung ist dabei eher unerwünscht.

Bemerkung 5.1 Ein wesentlicher Nachteil der hier vorgestellten DIRK-Verfahren ist die wegen $a_{11} \neq 0$ geringe Stufenordnung von $q=1$, siehe Definition 2.3 und Lemma 1 in [61]. Diese führt zu einer Reduktion der Konvergenzordnung für die algebraischen Variablen. Um die Stufenordnung anzuheben, wird bei der Konstruktion neuer Verfahren meist eine zusätzliche explizite erste Stufe hinzugefügt. Das heißt, es wird $Y_{1 n}=y_{n}$, $c_{1}=0$ und $a_{1 k}=0$ für $k=1, \ldots, s$ gesetzt, wodurch eine Stufenordnung von $q=2$ möglich ist. Für diese Verfahren wird auch die Bezeichnung ESDIRK-Verfahren (Explicit first Stage DIRK-Verfahren) verwendet. Da $A$ dann jedoch nicht invertierbar ist, kann das Verfahren nicht gemäß (5.13) umgestellt werden. Solche Verfahren werden in dieser Arbeit nicht weiter untersucht. Hinweise zur Konstruktion, Beispiele und ein Vergleich sind z.B. in [61] zu finden.

\subsubsection{Die Radau-IIA-Verfahren}

Um die Einschränkung der niedrigen Stufenordnung von DIRK-Verfahren zu umgehen, können auch voll implizite Runge-Kutta-Verfahren betrachtet werden. Die hier vorgestellte Verfahren basieren auf Radau-Quadraturformeln, deren Stützstellen $c_{1}, \ldots, c_{s}$ den geordneten Nullstellen von

$$
\frac{d^{s-1}}{d x^{s-1}}\left(x^{s-1}(x-1)^{s}\right)
$$

entsprechen. Es handelt sich um Kollokations-Verfahren, d.h., es wird ein Polynom $\hat{y}$ vom Grad $s$ durch $y_{n}$ gelegt und gefordert, dass die Differentialgleichung (5.1) in den Stellen $t_{n}+c_{k} \tau_{n}$ für $k=1, \ldots, s$ efüllt ist. Es ist dann $y_{n+1}=\hat{y}\left(t_{n+1}\right)$. Nach Konstruktion ist $c_{s}=1$ und wegen der zur Konstruktion verwendeten Ordnungsbedingungen handelt es sich um steif-stabile Verfahren der Ordnung $2 s-1$ (siehe Theorem IV.5.3 in [31]). Außerdem sind die Verfahren B-stabil und algebraisch stabil (siehe Theorem IV.12.9 in [31]) und haben sehr gute Eigenschaften für sehr steife Probleme, sowie DAE Probleme mit Index 1 oder 2. Die globalen Fehlerordnungen sind in Tabelle 5.2 angegeben (siehe Tabellen IV.15.1, VI.2.1 und VI.7.1 in [31]).

Beim einstufigen Verfahren handelt es sich um das implizite Euler-Verfahren. Die zweibzw. dreistufigen Radau-IIA-Verfahren sind durch folgende Butcher-Schemata gegeben: 


\begin{tabular}{ccccccc}
\hline & steif, $\zeta=\tau \lambda \rightarrow \infty$ & \multicolumn{2}{c}{ Index 1} & & \multicolumn{2}{c}{ Index 2} \\
\cline { 3 - 4 } Stufen & $\mathrm{y}$ & $\mathrm{y}$ & $\mathrm{z}$ & $\mathrm{y}$ & $\mathrm{z}$ \\
\hline $\mathrm{s}$ & $\zeta^{-1} \tau^{s+1}$ & $\tau^{2 s-1}$ & $\tau^{2 s-1}$ & $\tau^{2 s-1}$ & $\tau^{s}$ \\
2 & $\zeta^{-1} \tau^{3}$ & $\tau^{3}$ & $\tau^{3}$ & $\tau^{3}$ & $\tau^{2}$ \\
\hline
\end{tabular}

Tabelle 5.2: globale Fehlerordnungen der differentiellen Variablen $y$ und der algebraischen Variablen $z$ für Radau-IIA-Verfahren.

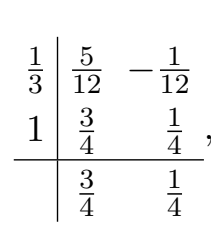

$$
\begin{array}{c|ccc}
\frac{4-\sqrt{6}}{10} & \frac{88-7 \sqrt{6}}{360} & \frac{296-169 \sqrt{6}}{1800} & \frac{-2+3 \sqrt{6}}{225} \\
\frac{4+\sqrt{6}}{10} & \frac{296+169 \sqrt{6}}{1800} & \frac{88+7 \sqrt{6}}{360} & \frac{-2-3 \sqrt{6}}{225} \\
1 & \frac{16-\sqrt{6}}{36} & \frac{16+\sqrt{6}}{36} & \frac{1}{9} \\
\hline & \frac{16-\sqrt{6}}{36} & \frac{16+\sqrt{6}}{36} & \frac{1}{9}
\end{array}
$$

Eine Umformung gemäß (5.13) führt für das zweistufige Verfahren zu

$$
\frac{1}{\tau_{n}}\left(\begin{array}{rr}
\frac{3}{2} & \frac{1}{2} \\
-\frac{9}{2} & \frac{5}{2}
\end{array}\right)\left(\begin{array}{l}
Y_{1 n} \\
Y_{2 n}
\end{array}\right)=\left(\begin{array}{l}
f\left(t_{n}+c_{1} \tau_{n}, Y_{1 n}, Z_{1 n}\right) \\
f\left(t_{n}+c_{2} \tau_{n}, Y_{2 n}, Z_{2 n}\right)
\end{array}\right)+\frac{1}{\tau_{n}}\left(\begin{array}{r}
2 y_{n} \\
-2 y_{n}
\end{array}\right), \quad 0=\left(\begin{array}{l}
g\left(Y_{1 n}\right) \\
g\left(Y_{2 n}\right)
\end{array}\right) .
$$

Hier treten die Differenzenquotienten als Ableitungen des Kollokationspolynoms auf, was auch der ursrünglichen Konstruktion entspricht. Dieses System wird durch ein relaxiertes Einzelschrittverfahren (SOR) gelöst.

$$
\begin{aligned}
\frac{3}{2} \widetilde{Y}_{1 n}^{k+1} & =\tau_{n} f\left(t_{n}+c_{1} \tau_{n}, Y_{1 n}^{k} ; \widetilde{Y}_{1 n}^{k+1}, Z_{1 n}^{k+1}\right)-\frac{1}{2} Y_{2 n}^{k}+2 y_{n}, & & =g\left(\widetilde{Y}_{1 n}^{k+1}\right), \\
Y_{1 n}^{k+1} & =\omega \widetilde{Y}_{1 n}^{k+1}+(1-\omega) Y_{1 n}^{k+1}, & & \\
\frac{5}{2} \widetilde{Y}_{2 n}^{k+1} & =\tau_{n} f\left(t_{n}+c_{2} \tau_{n}, Y_{2 n}^{k} ; \widetilde{Y}_{2 n}^{k+1}, Z_{2 n}^{k+1}\right)+\frac{9}{2} Y_{1 n}^{k+1}-2 y_{n}, & & 0=g\left(\widetilde{Y}_{2 n}^{k+1}\right), \\
Y_{2 n}^{k+1} & =\omega \widetilde{Y}_{2 n}^{k+1}+(1-\omega) Y_{2 n}^{k+1} . & &
\end{aligned}
$$

Als Startwerte $Y_{1 n}^{0}, Y_{2 n}^{0}$ für die Iteration werden die extrapolierten Werte des Kollokationspolynoms vom letzten Zeitschritt verwendet. Im ersten Iterationsschritt wird $\omega=1$ gesetzt, um die linearen algebraischen Nebenbedingungen für Randwerte und Divergenzfreiheit herzustellen. Falls der Zeitschritt klein genug ist, wird die Konvergenzrate durch die Kopplungsmatrix bestimmt. Ohne Relaxation beträgt die Kontraktionszahl $q=0.6$. Bei für das SOR-Verfahren optimalem Parameter $\omega^{*}=\frac{1}{3}(\sqrt{160}-10) \approx 0.883$ nach Satz 6.17 in [49] beträgt die Kontraktionszahl $q^{*}=\frac{1}{3}(13-\sqrt{160}) \approx 0.117$. Die Relaxation beschleunigt das Verfahren also um bis zu einen Faktor $\log \left(q^{*}\right) / \log (q) \approx 4$.2. Für kleine Zeitschritte sind diese Werte tatsächlich, wie in Abbildung 5.1 gezeigt, numerisch zu beobachten. Da die Nichtlinearität simultan iteriert wird, kann die Konvergenzrate jedoch schlechter ausfallen, was für größere Zeitschritte zu beobachten ist. In diesem Fall verursacht die Kopplung nur geringe Zusatzkosten gegenüber DIRK-Verfahren, bei denen sequentiell die einzelnen Stufen gelöst werden. Eine Untersuchung der Iterationszahlen findet zusammen mit einer Konvergenzstudie in Kapitel 5 statt. 


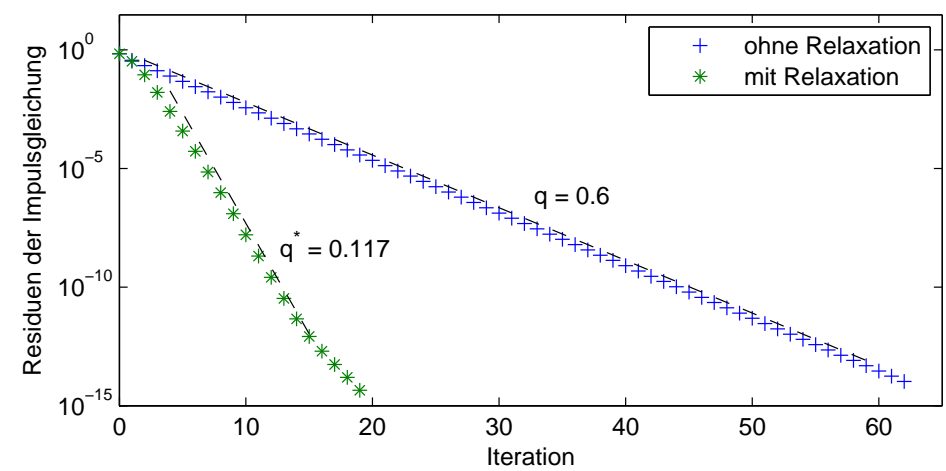

Abbildung 5.1: Residuenentwicklung während des relaxierten Einzelschrittverfahrens für das zweistufige Radau-IIA-Verfahren (einzelner Zeitschritt der Simulation einer dreidimensionalen thermisch getriebenen Hohlraumströmung mit Turbulenzmodell, siehe Kapitel 8).

Für das dreistufige Verfahren kann analog vorgegangen werden. Der optimale Relaxationsparameter ist $\omega^{*} \approx 0.896$ und die optimale Kontraktionszahl $q^{*} \approx 0.325 \mathrm{im}$ Gegensatz zu $q \approx 0.972$ ohne Relaxation. Obwohl das einer Beschleunigung um den Faktor $\log \left(q^{*}\right) / \log (q) \approx 39.6$ entspricht, ist die Konvergenz langsam. Aufwendigere Lösungsverfahren für das gekoppelte System, wie z.B. Quasi-Newton-Verfahren, wurden nicht untersucht, werden für praktische Anwendungen bei diesen Verfahren jedoch empfohlen.

\subsubsection{IMEX Runge-Kutta Verfahren}

Auch bei Runge-Kutta Verfahren können einzelne Terme mit unterschiedlichen Verfahren behandelt werden. Dabei werden - wie bei den Mehrschrittverfahren - für steife Terme meist implizite Verfahren mit guten Stabilitätseigenschaften und für z.B. nicht-steife und nicht-lineare Terme explizite Verfahren verwendet, um die Nichtlinearität zu umgehen. Solche Verfahren werden IMEX (implicit/explicit) oder auch additive Runge-Kutta Verfahren genannt. In [4] werden einfache IMEX Runge-Kutta-Verfahren für partielle Differentialgleichungen basierend auf DIRK-Verfahren vorgestellt. Durch die Verwendung von DIRK-Verfahren für den impliziten Teil leiden aber auch diese unter Ordnungsreduktion für algebraische Variablen von DAE Problemen. Dieser Nachteil wird bei den in [39] konstruierten Verfahren durch die Verwendung von ESDIRK-Verfahren (siehe Bemerkung 5.1) vermieden. Ein Überblick über die theoretischen Eigenschaften einer großen Auswahl solcher Verfahren ist ebenfalls in [39, Appendix B] zu finden. Einige IMEX Runge-Kutta-Verfahren können auch als Anwendung einer einzelnen Fixpunktiteration für die Stufen eines impliziten Verfahrens mit speziellen extrapolierten Startwerten, also eine Art Prädiktor-Korrektor-Verfahren, interpretiert werden. Das IMEX DIRK $(2,2)$ Verfahren (siehe Abschnitt 2.6 in Ref. [4]) wurde auf diese Weise implementiert. 


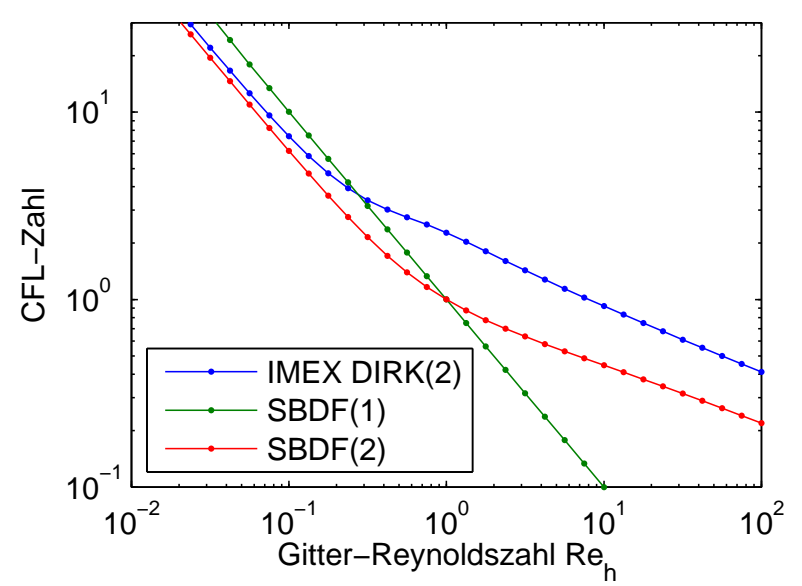

Abbildung 5.2: Zeitschrittweitenbeschränkung in Abhängigkeit von der Gitter-Reynoldszahl für ausgewählte IMEX-Verfahren.

\subsection{Stabilitätsgebiete und Zeitschrittweitenbeschränkung}

In Abschnitt 2.6 wurden die spektralen Eigenschaften einer Finite-ElementeDiskretisierung eines eindimensionalen Testproblems untersucht. Eine wichtige Eigenschaft der Eigenwerte war, dass sie alle negativen Realteil besitzen, was dazu führt, dass die Energie der semi-diskreten Lösungen abklingt. Wir bezeichnen mit $R(z=(\alpha+i \beta) \tau):=y_{1}$ die Lösung eines Zeitschrittverfahrens nach einem Zeitschritt der Länge $\tau$ angewendet auf das Testproblem

$$
y^{\prime}=a y+i b y, \quad y(0)=1
$$

mit $a, b \in \mathbb{R}$. Die Menge aller komplexen Zahlen $z$, für die $|R(z)| \leq 1$ ist, nennen wir Stabilitätsgebiet des Verfahrens. In Abbildung 5.3 sind die Stabilitätsgebiete verschiedener Verfahren abgebildet.

Der größte Zeitschritt $\tau_{\max }$ den ein Zeitschrittverfahren machen kann, so dass alle Eigenwerte der Diskretisierung des Differentialoperators im Stabilitätsgebiet des Verfahren liegen, liefert die CFL-Zahl $\frac{\tau_{\max } b}{h}$. Sie hängt somit sowohl von der räumlichen und zeitlichen Diskretisierung, als auch von der Gitter-Reynoldszahl $\operatorname{Re}_{h}$ ab. Für die $A$-stabilen impliziten Verfahren ist CFL $=\infty$, da die Stabilitätsgebiete die komplette Halbebene $\mathbb{C}_{-}=\{z \in \mathbb{C} \mid \operatorname{real}(z)<0\}$ umfassen. In Abbildung 5.2 sind für ausgewählte Verfahren die CFL-Zahlen in Abhängigkeit von $\mathrm{Re}_{h}$ aufgetragen. Bei kleinen Gitter-Reynoldszahlen unterliegen alle Verfahren ähnlichen Zeitschrittweitenbeschränkungen, bei großen GitterReynoldszahlen erlauben die Verfahren zweiter Ordnung deutlich größere Zeitschritte. 

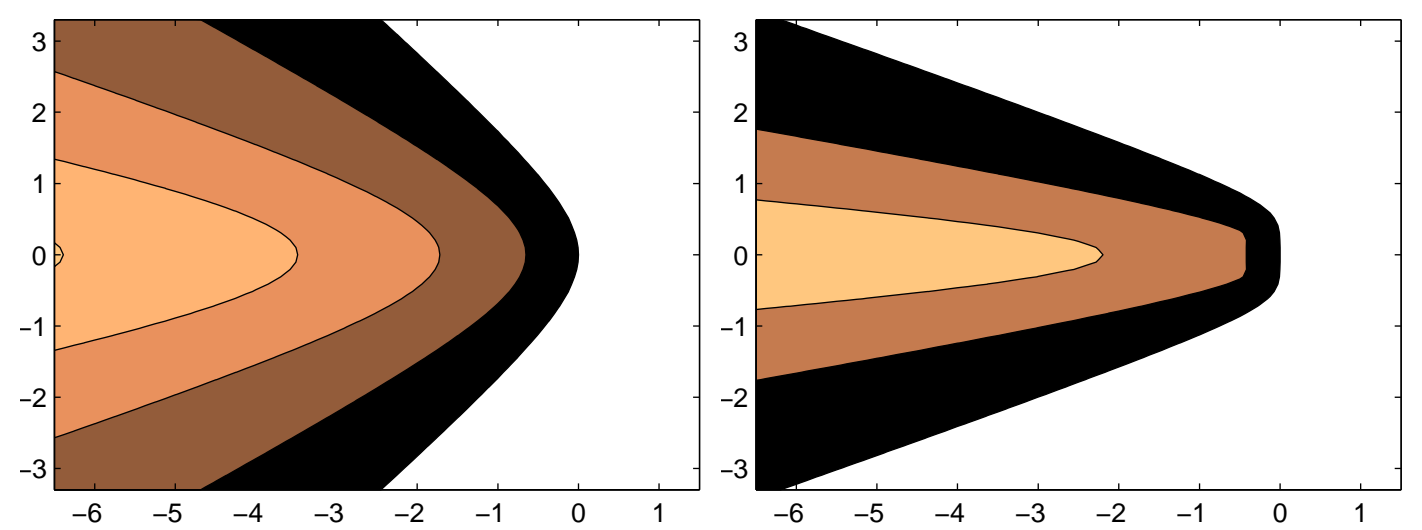

(a) IMEX SBDF Verfahren erster Ordnung (links) und zweiter Ordnung (rechts).
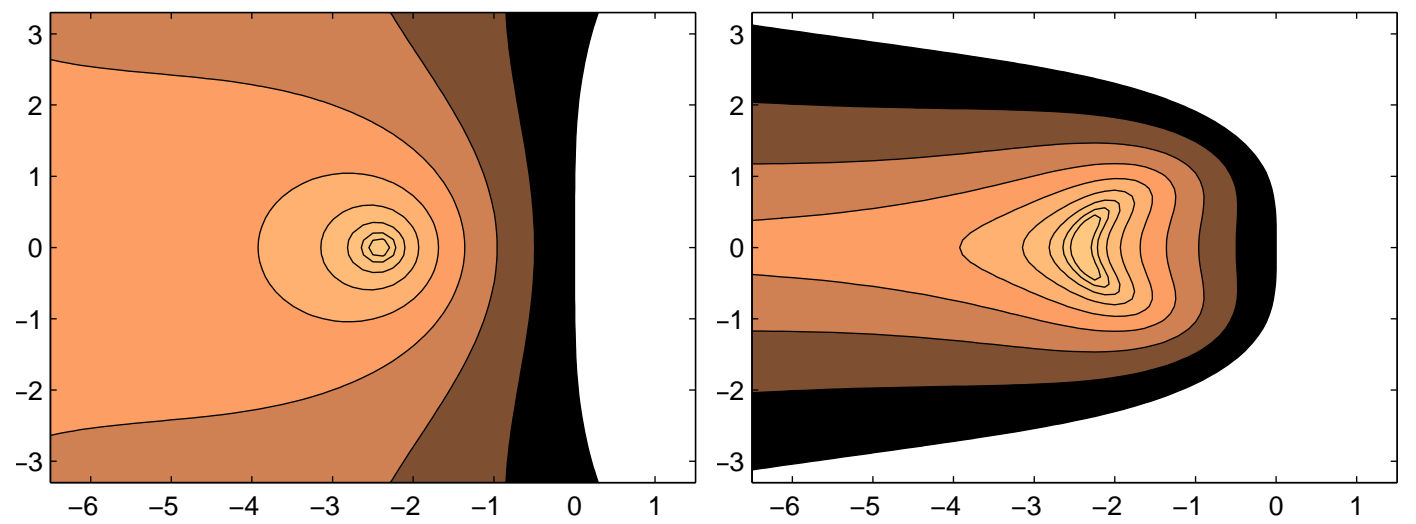

(b) Implizites (links) und IMEX (rechts) DIRK Verfahren zweiter Ordnung.
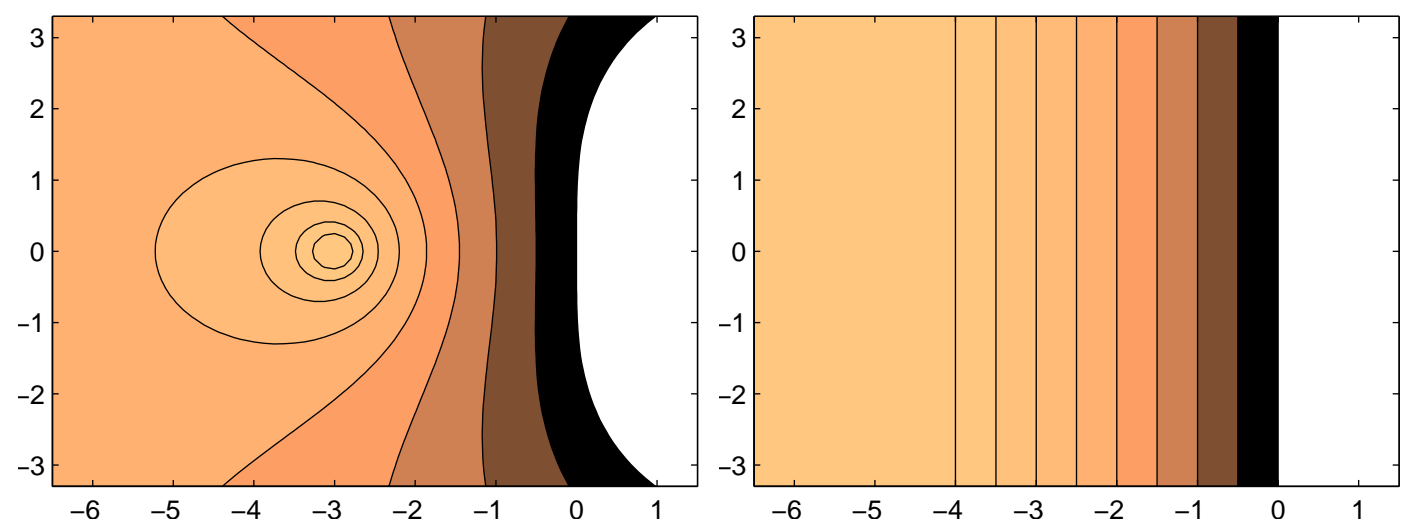

(c) Radau-IIA Verfahren (links), 'exaktes' Verfahren (rechts).

Abbildung 5.3: Stabilitätsgebiete verschiedener Zeitschrittverfahren. 


\begin{tabular}{lcccccc}
\hline Verfahren & Schritte & Stufen & nichtlinear & gekoppelt & Ordnung & Index 2 \\
\hline Radau-IIA & 1 & $\mathrm{~s}$ & $\checkmark$ & $\checkmark$ & $h^{2 s-1}$ & $h^{s}$ \\
DIRK & 1 & $\mathrm{~s}$ & $\checkmark$ & & $h^{s}$ & $h^{1}$ \\
BDF & $\mathrm{k}$ & 1 & $\checkmark$ & & $h^{k}$ & $h^{k}$ \\
sBDF & $\mathrm{k}$ & 1 & & & $h^{k}$ & $h^{k}$ \\
\hline
\end{tabular}

Tabelle 5.3: Eigenschaften der verschiedenen Verfahren.

\subsection{Stetige Ausgabe}

In der bisherigen Darstellung wurden die Zeitdiskretisierungsverfahren lediglich dazu verwendet, Näherungslösungen auf dem diskreten Zeitgitter $t_{0}<t_{1}<\cdots<t_{N}=T$ zu berechnen. Für einige Anwendungen kann jedoch eine kontinuierliche Lösung (dense output) erforderlich sein, um z.B. bestimmte Ereignisse zeitlich genau bestimmen zu können (event location), ohne unverhältnismäßig kleine Zeitschritte machen zu müssen. Da in jedem Gitterpunkt die Approximation der Lösung $y$ und mit $f(t, y) \approx y^{\prime}$ auch eine Näherung der Ableitung vorliegt, kann z.B. eine kubische Hermite-Interpolation angewendet werden, um eine Lösung für beliebige Zeitpunkte im Intervall $\left[t_{0}, T\right]$ zu erhalten. Dabei werden jedoch viele im Verfahren vorhandene Informationen nicht berücksichtigt. Bei den Kollokationsverfahren (BDF, Radau-IIA) bieten z.B. die Kollokationspolynome eine einfache und effiziente Möglichkeit, die Lösung über den ganzen Zeitschritt zu interpolieren.

\subsection{Zusammenfassung}

In diesem Kapitel wurden Verfahren zur zeitlichen Diskretisierung unterschiedlicher Komplexität vorgestellt. Einige ihrer grundlegenden Eigenschaften sind in Tabelle 5.3 zusammengefasst. Die sBDF-Verfahren zeichnen sich dadurch aus, dass diese auch die Linearisierung des Problems bereits beinhalten, was jedoch die Stabilität und Diskretisierungsfehler beeinträchtigt. Die impliziten BDF-Verfahren besitzen sehr gut Stabilitätseigenschaften, erfordern jedoch ein Iterationsverfahren für die Nichtlinearität. Bei den mehrstufigen Runge-Kutta-Verfahren erlauben die DIRK-Verfahren eine effiziente Lösung des gestaffelten nicht-linearen Gleichungssystems, sie leiden jedoch unter dem Effekt der Ordnungsreduktion für algebraische Variable. Die Radau-IIA-Verfahren, die wie die BDF-Verfahren auf Kollokation basieren, haben von den vorgestellten Verfahren die besten Konvergenzeigenschaften und ebenfalls sehr gute Stabilitätseigenschaften. Sie sind aber auch die aufwendigsten Verfahren.

Im nächsten Kapitel werden die Eigenschaften der Verfahren an einem zeitabhängigen Benchmark-Problem numerisch untersucht. Insbesondere wird überprüft, ob die aufwendigeren nicht-linearen und mehrstufigen Verfahren bessere Eigenschaften als die einfa- 
cheren Verfahren aufweisen.

Alle vorgestellten Verfahren wurden auf einheitliche Gleichungen oder Gleichungssysteme der Form (5.13) zurückgeführt und in dieser Form unabhängig von dem zugrundeliegenden Differentialgleichungssystem und desser räumlicher Diskretisierung implementiert. Diese Implementierung wurde z.B. auch für die numerische Untersuchungen von zerfallender homogener isotroper Turbulenz und von turbulenten Kanalströmungen in [62] verwendet. 


\section{Kapitel 6}

\section{Umströmung eines zweidimensionalen Zylinders}

Ein wichtiges physikalisches Phänomen bei der Umströmung von Körpern ist die rückseitige Wirbelablösung oder allgemein Strömungsablösung. Abgelöste Wirbel werden von der Strömung weiter transportiert und bilden eine sogenannte Kármánsche Wirbelstraße - nach Theodore von Kármán. Die charakteristische Frequenz $f$ der Wirbelablösung

$$
f=\operatorname{Sr} \frac{v}{d}
$$

wird durch die Strouhal-Zahl Sr, die Anströmgeschwindigkeit $v$ und eine charakteristische Abmessung $d$ des Körpers angegeben. Für einen frei angeströmten Zylinder ist $\mathrm{Sr} \approx 0.2$ und $d$ der Durchmesser. Bei kleinem Durchmesser und hohen Geschwindigkeiten können charakteristische Pfeiftöne entstehen, welche z.B. im Automobilbau den Fahrkomfort negativ beeinflussen können. Ein anderes Problem kann entstehen, wenn die Ablösefrequenz mit einer Eigenfrequenz des Körpers zusammenfällt. Dieser kann dann in Schwingung geraten, was bei heutzutage dynamisch ausgelegten Hochhäusern oder Hängebrücken berücksichtigt werden muss. Die korrekte Simulation von Wirbelablösung oder Strömungsablösung ist daher ein wichtiges Kriterium für numerische Methoden.

Weitere Kenngrößen im Zusammenhang mit umströmten Körpern sind der Auftriebsbeiwert $c_{\mathrm{a}}$ und der Widerstandsbeiwert $c_{\mathrm{w}}$. Diese spielen z.B. in der Aerodynamik von Tragflächen oder auch beim Design von Automobilen eine wichtige Rolle. Häufiges Ziel ist es, den Widerstandsbeiwert zu minimieren und gleichzeitig bestimmte Vorgaben für den Auftriebsbeiwert einzuhalten. Außerdem kann noch der Drehmomentbeiwert z.B. bei der Umströmung von Rotoren oder Tragflächen eine Rolle spielen. Der Drehmomentbeiwert wird in diesem Kapitel allerdings nicht weiter betrachtet.

Das folgende Benchmarkproblem eines umströmten Zylinders wurde im Rahmen der DFG Schwerpunktprogramme "Flow Simulation with High-Performance Computers" definiert und ist darauf ausgelegt, Auftriebs- und Widerstandsbeiwerte sowie den zeitlich 
korrekten Verlauf der Wirbelablösung zu bestimmen. Ergebnisse aller teilnehmenden Gruppen wurden in [71] ausgewertet. Neben Varianten mit stationärer und periodischer Lösung ist auch ein Problem mit zeitabhängiger Einströmbedingung beschrieben. Diese schwierigste Variante des Problems werden wir im Folgenden betrachten.

Bemerkung 6.1 Wir untersuchen dieses laminare und isotherme Benchmarkproblem um zunächst einzelne Aspekte der Diskretisierung isoliert zu betrachten. Da es sich um ein zeitabhängiges Benchmarkproblem handelt, kann speziell die Konvergenz der Zeitschrittverfahren gut untersucht werden. Die später folgenden nicht-isothermen Probleme haben entweder stationäre oder statistisch stationäre Lösungen, so dass diese Möglichkeit dort nicht direkt gegeben ist. Außerdem handelt es sich beim umströmten Zylinder um eine nicht-triviale Geometrie mit krummlinigen Rändern. Die Betrachtung dieses Problems ergänzt somit die numerischen Untersuchungen der beiden folgenden Kapitel zu nicht-isothermen Problemen.

\subsection{Benchmarkbeschreibung}

Das Gebiet $\Omega$ ist in Abbildung 6.1 dargestellt. Es handelt sich um einen Kanal der Länge $L=2.2 \mathrm{~m}$ und der Höhe $H=0.41 \mathrm{~m}$ mit einer kreisförmigen Aussparung mit Durchmesser $D=0.1 \mathrm{~m}$ im vorderen Teil. Die Aussparung liegt nicht in der Mitte des Kanals, so dass es keine Symmetrieebenen gibt.

Es wird das Zeitintervall $t \in(0,8]$ betrachtet und als Startwert $\mathbf{u}=\mathbf{0}$ verwendet. Die rechte Seite der Impulsgleichung ist ebenfalls $\mathbf{f}=\mathbf{0}$ und die Einströmbedingung ist durch

$$
\mathbf{u}\left(t ;(0, y)^{\top}\right)=\mathbf{u}\left(t ;(2.2, y)^{\top}\right)=\frac{4 U_{m}}{H^{2}} \sin (\pi t / 8 \mathrm{~s})\left(\begin{array}{c}
y(H-y) \\
0
\end{array}\right), \quad U_{m}=1.5 \mathrm{~ms}^{-1}
$$

gegeben. Die mittlere Einströmgeschwindigkeit beträgt also $\bar{U}(t)=\frac{2}{3} \sin (\pi t / 8) U_{m}$. Die verwendete Ausströmbedingung hat wegen des ausreichend langen Kanals kaum Einfluss auf die Lösung im Bereich des Zylinders.

Für die auf der mittleren Einströmgeschwindigkeit, dem Zylinderdurchmesser und der kinematischen Viskosität $\nu=10^{-3} \mathrm{~m}^{2} \mathrm{~s}^{-1}$ basierende Reynoldszahl gilt $0 \leq R e(t)=$ $\frac{\bar{U} D}{\nu} \leq 100$. Die Strömung ist vollständig laminar.

\subsection{Benchmarkgrößen}

Wichtige Kenngrößen umströmter Objekte im Fall isothermer Strömungen sind der Widerstandsbeiwert $c_{\mathrm{w}}$ und der Auftriebsbeiwert $c_{\mathrm{a}}$. Diese setzen sich aus viskosen Reibungskräften tangential zur Objektoberfläche und aus Druckkräften normal zur Objektoberfläche zusammen. Die Kräfte werden durch das Geschwindigkeitsquadrat und den 


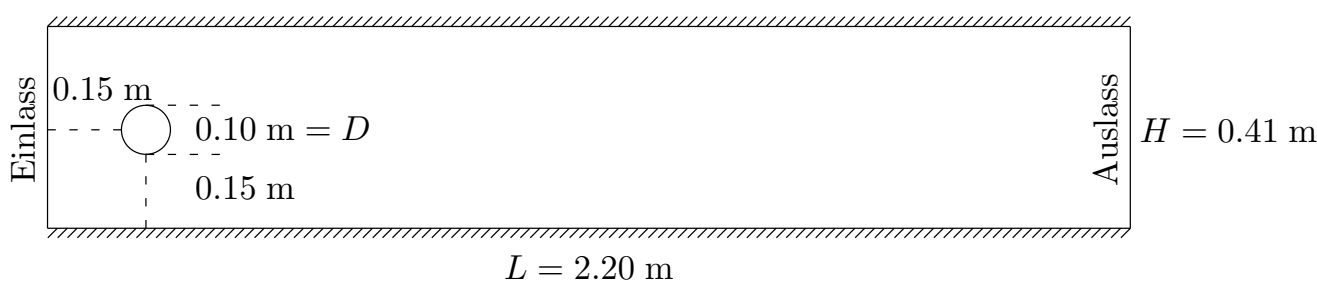

Abbildung 6.1: Geometrie des umströmten Zylinders im Kanal.

Objektquerschnitt normiert.

$$
c_{\mathrm{w}}:=\frac{2}{\varrho \bar{U}^{2} D} \int_{S}\left(\varrho \nu \frac{\partial v_{t}}{\partial \mathbf{n}} n_{y}-p n_{x}\right) d S, \quad c_{\mathrm{a}}:=-\frac{2}{\varrho \bar{U}^{2} D} \int_{S}\left(\varrho \nu \frac{\partial v_{t}}{\partial \mathbf{n}} n_{x}+p n_{y}\right) d S .
$$

Dabei bezeichnet $S$ den Kreisrand, $\mathbf{n}=\left(n_{x}, n_{y}\right)^{\top}$ den Normaleneinheitsvektor an $S$, $\mathbf{t}=\mathbf{n}^{\perp}=\left(n_{y},-n_{x}\right)^{\top}$ den Tangentialvektor und $v_{t}=\mathbf{u} \cdot \mathbf{t}$ die Tangentialgeschwindigkeit. Eine ausführliche Betrachtung zur Berechnung von $c_{\mathrm{w}}$ und $c_{\mathrm{a}}$ ist in [35] ab Seite $142 \mathrm{zu}$ finden. Es ist nach Definition:

$$
\frac{\partial v_{t}}{\partial \mathbf{n}}:=\left(u_{1 x} n_{y}-u_{2 x} n_{x}\right) n_{x}+\left(u_{1 y} n_{y}-u_{2 y} n_{x}\right) n_{y} .
$$

Unter Verwendung der Eigenschaft, dass $\mathbf{u}$ in beiden Komponenten auf dem Kreisrand konstant ist und somit die Tangentialableitungen

$$
\frac{\partial u_{1}}{\partial \mathbf{t}}=u_{1 x} n_{y}-u_{1 y} n_{x}=0, \quad \frac{\partial u_{2}}{\partial \mathbf{t}}=u_{2 x} n_{y}-u_{2 y} n_{x}=0
$$

verschwinden, lässt sich (6.2) zu

$$
\frac{\partial v_{t}}{\partial \mathbf{n}}=\left(u_{1 y}-u_{2 x}\right)\left(n_{x}^{2}+n_{y}^{2}\right)=u_{1 y}-u_{2 x}
$$

vereinfachen. Diese Identität gilt auf jedem glatten Teilstück der Gitterapproximation von $S_{h}$. Die Integrale (6.1) können für diskrete Lösungen unter Verwendung numerischer Quadraturformeln und unter Berücksichtigung der krummlinigen Ränder mit hoher Genauigkeit berechnet werden. Im Gegensatz zu Resultaten in [35] ist dabei bis auf den numerischen Rechenfehler der verwendeten Gleitkommatypen kein Unterschied zwischen den Formeln (6.2) und (6.3) feststellbar. Eine mögliche Ursache für die Abweichungen in [35] ist eine unzureichende Berücksichtigung der krummlinigen Ränder in den numerischen Quadraturformeln.

Alternativ können die Oberflächenintegrale (6.1) in Volumenintegrale transformiert werden. Dazu bilden wir das $L^{2}$-Skalarprodukt der Impulsgleichung mit speziellen Testfunktionen und verwenden partielle Integration:

$$
\begin{aligned}
& c_{\mathrm{w}}=-\frac{2}{\bar{U}^{2} D}\left[\left(\mathbf{u}_{t}, \mathbf{v}_{\mathrm{w}}\right)+\left(\nu \nabla \mathbf{u}, \nabla \mathbf{v}_{\mathrm{w}}\right)+\left((\mathbf{u} \cdot \nabla) \mathbf{u}, \mathbf{v}_{\mathrm{w}}\right)-\left(p, \nabla \cdot \mathbf{v}_{\mathrm{w}}\right)\right], \\
& c_{\mathrm{a}}=-\frac{2}{\bar{U}^{2} D}\left[\left(\mathbf{u}_{t}, \mathbf{v}_{\mathrm{a}}\right)+\left(\nu \nabla \mathbf{u}, \nabla \mathbf{v}_{\mathrm{a}}\right)+\left((\mathbf{u} \cdot \nabla) \mathbf{u}, \mathbf{v}_{\mathrm{a}}\right)-\left(p, \nabla \cdot \mathbf{v}_{\mathrm{a}}\right)\right] .
\end{aligned}
$$


Dabei sind $\mathbf{v}_{\mathrm{w}}, \mathbf{v}_{\mathrm{a}} \in\left[H^{1}(\Omega)\right]^{2}$ beliebige Funktionen mit $\left.\mathbf{v}_{\mathrm{w}}\right|_{S}=(1,0)^{T}$ bzw. $\left.\mathbf{v}_{\mathrm{a}}\right|_{S}=$ $(0,1)^{T}$ und $\left.\mathbf{v}_{\mathrm{w}}\right|_{\partial \Omega \backslash S}=\left.\mathbf{v}_{\mathrm{a}}\right|_{\partial \Omega \backslash S}=\mathbf{0}$. In praktischen Versuchen zeigte sich jedoch, dass die Werte stark von den konkret verwendeten Testfunktionen abhängen. Ein weiterer Nachteil dieser Berechnungsvorschrift ist die Abhängigkeit von der Approximation der Zeitableitung $\partial_{t} \mathbf{u}$. Außerdem können weitere nicht-konsistente Modellterme (Stabilisierung, Turbulenzmodell) Auswirkungen auf die Berechnung haben. Die Einschätzung aus [35], dass die Formeln (6.4) genauer und weniger sensitiv bezüglich der Diskretisierung als die Formeln (6.1) sind, konnte praktisch nicht bestätigt werden. Daher werden in den folgenden Untersuchungen zur Berechnung des Widerstands- und Auftriebsbeiwertes die Formeln (6.1) zusammen mit der Vereinfachung (6.3) verwendet.

Wesentlichen Einfluss auf den Widerstandskoeffizienten hat die Druckdifferenz zwischen der Vorder- und Rückseite des Kreises. Dieser Druckabfall (pressure drop)

$$
\Delta p(t):=p(0.15,0.2, t)-p(0.25,0.2, t)
$$

bildet die letzte Kenngröße für die Testrechnungen. Für unstetige Druckdiskretisierung werden die Werte von den angrenzenden Zellen gemittelt.

Nach der Auswertung von Benchmarkrechungen verschiedener Gruppen wurden in [71] folgende Intervalle für die Kenngrößen bestimmt:

$$
c_{\mathrm{w}, \text { max }}^{\mathrm{ref}} \in[2.93,2.97], \quad c_{\mathrm{a}, \max }^{\mathrm{ref}} \in[0.47,0.49], \quad \Delta p^{\mathrm{ref}} \in[-0.115,-0.105] .
$$

Viele Rechnungen lieferten trotz teils enormen Aufwands deutlich davon abweichende Werte. Meist ist dies auf einen Flaschenhals in der Güte der Diskretisierung zurückzuführen, wenn z.B. ein Gitter mit mehreren hunderttausend Knoten mit einem expliziten Euler-Verfahren kombiniert wird. In einer nachfolgenden Arbeit [37] wurden die Intervalle teils deutlich auf folgende Werte verkleinert

$$
\begin{aligned}
c_{\mathrm{w}, \max }^{\mathrm{J}} & \in[2.950921,2.950922], & & t_{\mathrm{w}, \max }^{\mathrm{J}} \approx 3.93625, \\
c_{\mathrm{a}, \max }^{\mathrm{J}} & \in[0.4779,0.4780], & & t_{\mathrm{a}, \max }^{\mathrm{J}} \approx 5.693125, \\
\Delta p^{\mathrm{J}}(8) & \in[-0.11155,-0.11165] . & &
\end{aligned}
$$

Dabei wurden Finite-Elemente-Verfahren zweiter Ordnung im Ort und ein fractional-step $\theta$-Schema sowie das Crank-Nicolson-Schema in der Zeit verwendet. Bei den angegebenen Werten handelt es sich um extrapolierte Werte.

Der zeitliche Verlauf der Kenngrößen einer Referenzrechnung ist in Abbildung 6.2 dargestellt. Es ist deutlich zu erkennen, wie der Widerstandsbeiwert und der Druckabfall hauptsächlich von der zeitlich variierenden Anströmgeschwindigkeit abhängen. Beide erreichen ihr Maximum etwa bei maximaler Anströmgeschwindigkeit zum Zeitpunkt $t=4$.

Der Auftriebsbeiwert zeigt ein deutlich interessanteres Verhalten. Schon bei niedriger Anströmgeschwindigkeit löst sich die Strömung an der Rückseite des Zylinders ab. Durch die Asymmetrie wird die Ablösung in Schwingung versetzt, die sich bis zur Wirbelablösung aufschaukelt. Dadurch entstehen Auftriebskräfte am Zylinder, die ihr Maximum etwa 

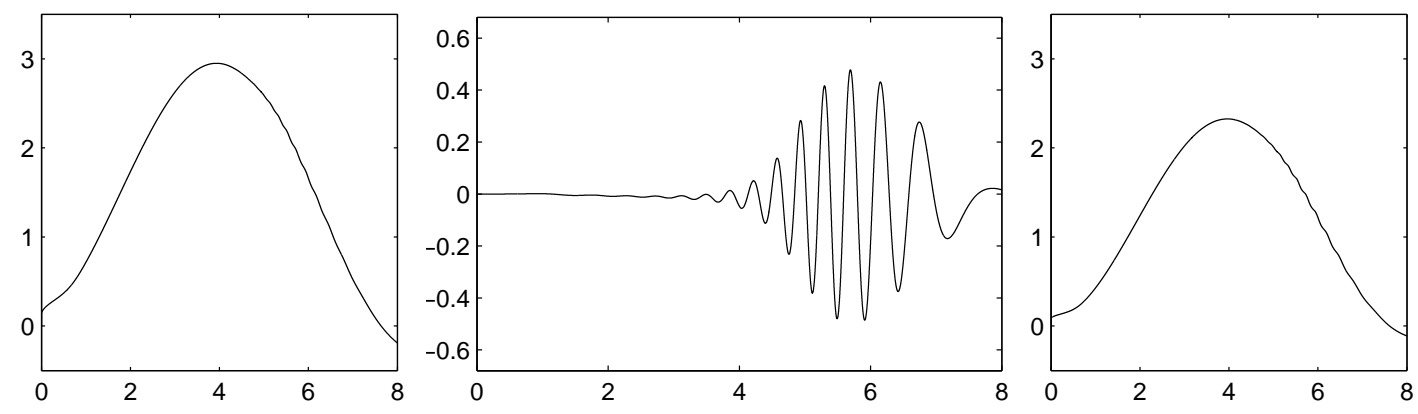

Abbildung 6.2: Widerstandsbeiwert (links), Auftriebsbeiwert (mitte) und Druckabfall (rechts) im zeitlichen Verlauf.

zum Zeitpunkt $t=5.7$ erreichen, aber deutlich schwächer als die Widerstandskräfte sind. Dieses komplexe Verhalten wird durch die Nichtlinearität des Konvektionsterms erzeugt und tritt bei Stokes-Strömungen nicht auf. Da nur im Auftriebsbeiwert die Dynamik der Wirbelablösung gut wiederzuerkennen ist, werden sich weitere qualitative Vergleiche meist auf den Auftriebsbeiwert beziehen.

Eine Abschätzung der Strouhal-Zahl Sr durch Messung des Abstands der beiden lokalen Minima des Auftriebsbeiwertes vor und nach dem Maximum ergibt

$$
\mathrm{Sr}=\frac{D f}{U} \approx \frac{0.1 \mathrm{~m} \cdot(5.911 \mathrm{~s}-5.490 \mathrm{~s})^{-1}}{\bar{U}((5.911 \mathrm{~s}+5.490 \mathrm{~s}) / 2)} \approx 0.303 .
$$

Dies liegt deutlich über dem für einen umströmten Zylinder angegebenen Literaturwert von $\mathrm{Sr} \in[0.14,0.21]$ nach [66, Tabelle 1.1] und hängt vermutlich mit dem Einfluss der horizontalen Kanalwände auf die Strömung sowie mit der für die Kanalmitte etwas zu niedrig angesetzten mittleren Strömungsgeschwindigkeit zusammen. Die maximale Strömungsgeschwindigkeit in der Kanalmitte ist gerade um $50 \%$ größer als die mittlere Strömungsgeschwindigkeit. Der Literaturwert bezieht sich hingegen auf eine freie Anströmung. In Benchmarkrechungen zum Kanal mit konstanter Anströmung wird ebenfalls ein Wert von $\mathrm{Sr} \approx 0.3$ beobachtet [71].

\subsection{Diskretisierung}

Das Grobgitter (Level $L=0$ ) wird in Abbildung 6.3 (oben) dargestellt und besteht aus 64 Zellen. Im hinteren Bereich des Kanals werden die Zellen etwas gestreckt, um einige Zellen einzusparen. So wie die Wahl der Randbedingung am Auslass hat dies kaum Einfluss auf die Strömung im Bereich des Zylinders. Außerdem ist der Zylinder von einem zusätzlichen Ring von Zellen umgeben, die dafür sorgen, dass beim Verfeinern ein annähernd isotropes Gitter entsteht.

Für an den Kreis angrenzende Zellen wird eine iso-parametrische Transformation verwendet, wie in den vergrößerten Ausschnitten zu sehen ist. Das ist insbesondere für 


\begin{tabular}{|l|l|l|l|l|l|l|l|l|l|l|}
\hline \\
\hline
\end{tabular}
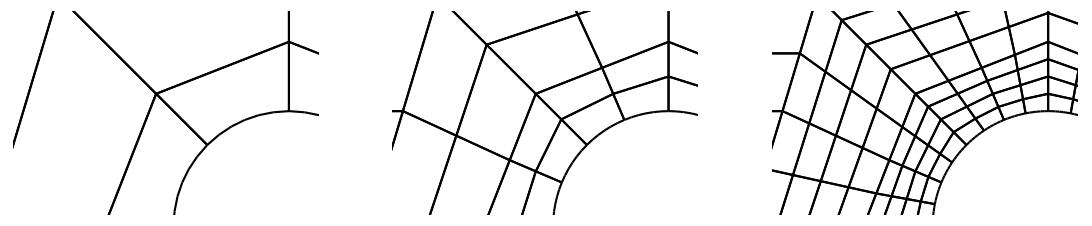

Abbildung 6.3: Ausgangsgitter und Ausschnitt um den Zylinder mit verschiedenen Verfeinerungsstufen und krummlinigen Rand.

hohe Elementordnung nötig, da sonst allein der Konsistenzfehler am Kreisrand den gesamten Diskretisierungsfehler dominiert. Die Auswertung von Benchmarkresultaten zum umströmten Zylinder in [71] hat gezeigt, dass nur Verfahren, die durchgängig eine Diskretisierung hoher Ordnung verwenden, effizient genaue Simulationen durchführen können.

Durch globales Verfeinern wird eine Gitterfamilie bis $L=4$ erzeugt. Beim Verfeinern werden neue Gitterpunkte, die zum Kreisrand $\partial S$ gehören, mit exakten Koordinaten versehen. Es handelt sich also nicht um eine echte konforme Gitterhierarchie. Auf diesen Gittern werden Taylor-Hood-Elementpaare bis zur vierten Ordnung verwendet. Die Anzahl der Zellen und Freiheitsgrade für die verschiedenen Verfeinerungsstufen und Elementordnungen ist in Tabelle 6.1 angegeben.

\begin{tabular}{|c|c|c|c|c|}
\hline \multirow[b]{2}{*}{ Level } & \multirow[b]{2}{*}{ Zellen } & $Q_{2} Q_{1}$ & $Q_{3} Q_{2}$ & \multirow{2}{*}{$\frac{Q_{4} Q_{3}}{\text { Geschw./ Druck }}$} \\
\hline & & Geschw./Druck & Geschw./Druck & \\
\hline 0 & 64 & $506 /$ & $1278 /$ & $2216 /$ \\
\hline 1 & & $2216 / \quad 29$ & $4860 / 110$ & $8528 / 2430$ \\
\hline 2 & & $8528 / 110$ & $18936 / 42$ & $33440 /$ \\
\hline 3 & & $3440 / 42$ & $74736 / 167$ & $132416 / 37368$ \\
\hline 4 & 16384 & $132416 / 16720$ & $296928 / 66208$ & $526976 / 148464$ \\
\hline
\end{tabular}

Tabelle 6.1: Anzahl der Freiheitsgrade für Geschwindigkeit und Druck für verschiedene Elementordnungen und Verfeinerungsstufen.

Alle Zeitschrittverfahren werden mit äquidistanten Zeitgittern verwendet, und die Kenngrößen zunächst nur zu vollen Zeitschritten ausgewertet. Um die Anzahl der Zeitschritte kurz angeben zu können, verwenden wir $M=\log _{2}(T / \tau)$. 


\subsection{Linearisierung}

Wie in Kapitel 5 besprochen, müssen für die voll impliziten Zeitschrittverfahren nichtlineare Probleme z.B. durch die im Folgenden genutzte Fixpunktiteration gelöst werden. In diesem Abschnitt untersuchen wir den Einfluss des nichtlinearen Residuums auf die Anzahl der benötigten Iterationsschritte und auf die Genauigkeit der zu berechnenden Kenngrößen. In den mit \#lp bezeichneten Spalten sind dazu zwei Zahlen angegeben, erstens die Anzahl der insgesamt vom Verfahren gelösten linearen Probleme und zweitens die durchschnittliche Anzahl linearer Probleme pro Zeitschritt.

Beim Vergleich des Aufwands werden wir nur die Anzahl der zu lösenden linearen Probleme heranziehen, da alle hier verwendeten Verfahren mit der gleichen Löserschnittstelle arbeiten und wir keine Annahmen über die speziellen Eigenschaften des verwendeten Lösers machen. Für die Testrechnungen wird meist ein direkter Löser für die linearen Probleme verwendet. An dieser Stelle sei jedoch darauf hingewiesen, dass z.B. die Zeitschrittweite ebenfalls Einfluss auf die Konvergenzrate eines iterativen Lösers hat und dass auch dort das Ziel-Residuum sorgfältig gewählt werden muss. Angegebene Residuen beziehen sich immer auf die euklidsche Norm des Residuenvektors. Es wird immer mindestens ein Linearisierungsschritt ausgeführt, um die neuen Randwerte aufzuerlegen und die Divergenzbedingung wiederherzustellen, auch wenn das Startresiduum schon die Vorgabe erfüllt.

Für den Test werden exemplarisch die Verfahren zweiter Ordnung (BDF2, DIRK2 und RadauIIA) auf Gitterlevel $L=3$ mit konstanter Zeitschrittzahl $T / \tau=4096$ betrachtet. $\mathrm{Zu}$ festen Zeitpunkten $t_{1}=3.9375$ (Zeitschritt 2016) und $t_{2}=5.6875$ (Zeitschritt 2912), die jeweils nahe der erwarteten Maxima liegen, sowie $t_{3}=8$ wird der Einfluss des Residuums auf den Auftriebs- und Widerstandskoeffizienten sowie den Druckabfall untersucht.

Das BDF-Verfahren benötigt im Vergleich die wenigsten Iterationsschritte und ist auch ohne Fixpunktiteration stabil. Schon mit einem einzelnen Linearisierungsschritt liegt das Residuum durchgehend unter $10^{-4}$. Das DIRK-Verfahren verhält sich ähnlich wie das BDF-Verfahren, benötigt aber etwas mehr als die erwartete doppelte Schrittzahl. Dies ist zum Teil auf die Qualität der Startwerte für die Iteration und zum Teil auf die unterschiedlichen Koeffizienten der Differenzenquotienten zurückzuführen. Für beide Verfahren werden wir als Zielresiduum $10^{-10}$ verwenden. Dies stimmt mit dem in [37] für ein fractional-step $\theta$-Schema und das Crank-Nicolson-Verfahren verwendeten Residuum überein.

Das Radau-IIA-Verfahren ist ohne Fixpunktiteration bei dieser Schrittweite nicht stabil, da es sich dann um ein explizites Verfahren handelt. Die Iterationszahlen liegen noch einmal deutlich über denen des ebenfalls zweistufigen DIRK-Verfahrens, da die Konvergenzrate insbesondere bei kleinen Residuen durch die Kopplung der beiden Stufen zusätzlich verringert wird. Um eine mit den anderen Verfahren vergleichbare Genauigkeit zu erreichen, werden wir für das Radau-IIA-Verfahren $10^{-8}$ als Zielresiduum verwenden. 
Kapitel 6: Umströmung eines zweidimensionalen Zylinders

\begin{tabular}{|c|c|c|c|c|c|}
\hline & Res. & $\# \mathrm{lp}$ & $c_{\mathrm{w}}\left(t_{1}=3.9375\right)$ & $c_{\mathrm{a}}\left(t_{2}=5.6875\right)$ & $\Delta p(8)$ \\
\hline \multirow[t]{6}{*}{ BDF2 } & - & $4096 / 1.00$ & 2.944451189 & 0.4794153626 & -0.1117227557 \\
\hline & $10^{-4}$ & $4096 / 1.00$ & 2.944451189 & 0.4794153626 & -0.1117227557 \\
\hline & $10^{-6}$ & $5995 / 1.46$ & 2.944441961 & 0.4747501611 & -0.1118463674 \\
\hline & $10^{-8}$ & $9905 / 2.42$ & 2.944415175 & 0.4717578723 & -0.1119053091 \\
\hline & $10^{-10}$ & $14927 / 3.64$ & 2.944414390 & 0.4716457298 & -0.1119078032 \\
\hline & $10^{-12}$ & $22219 / 5.42$ & 2.944414379 & 0.4716434617 & -0.1119078697 \\
\hline \multirow[t]{6}{*}{ DIRK2 } & - & $8192 / 2.00$ & 2.944692654 & 0.4618651442 & -0.1100359783 \\
\hline & $10^{-4}$ & $10569 / 2.58$ & 2.944692654 & 0.4672199232 & -0.1116711137 \\
\hline & $10^{-6}$ & $16822 / 4.11$ & 2.944384686 & 0.4742284144 & -0.1118335291 \\
\hline & $10^{-8}$ & $23687 / 5.78$ & 2.944382677 & 0.4738885527 & -0.1118346816 \\
\hline & $10^{-10}$ & $33797 / 8.25$ & 2.944382101 & 0.4738452714 & -0.1118348663 \\
\hline & $10^{-12}$ & $48871 / 11.9$ & 2.944382101 & 0.4738452457 & -0.1118348667 \\
\hline \multirow[t]{6}{*}{ Radau-IIA } & - & $8192 / 2.00$ & - & nicht stabil & - \\
\hline & $10^{-4}$ & $11678 / 2.85$ & 2.944417453 & 0.4733996754 & -0.1118485333 \\
\hline & $10^{-6}$ & $23782 / 5.81$ & 2.944418698 & 0.4738303100 & -0.1118368226 \\
\hline & $10^{-8}$ & $46162 / 11.3$ & 2.944419589 & 0.4741014404 & -0.1118238651 \\
\hline & $10^{-10}$ & $67132 / 16.4$ & 2.944419584 & 0.4741006554 & -0.1118238947 \\
\hline & $10^{-12}$ & $89054 / 21.7$ & 2.944419584 & 0.4741006466 & -0.1118238949 \\
\hline
\end{tabular}

Tabelle 6.2: Anzahl der Fixpunkt-Iterationsschritte und Kenngrößen für verschiedene Residuen auf Gitterlevel $L=3$ und mit $T / \tau=4096$ Zeitschritten.

Insgesamt ist zu beobachten, dass der Auftriebsbeiwert stärker von der Nichlinearität abhängt als die anderen Kenngrößen, da er deutlich durch die Wirbelablösung beeinflusst wird, während der Widerstandsbeiwert und der Druckabfall hauptsächlich von der Anströmgeschwindigkeit abhängen und weniger durch das nichtlineare Residuum beeinflusst werden.

\subsection{Konvergenzuntersuchung der zeitlichen Diskretisie- rung}

Die Konvergenz hinsichtlich der Zeitschrittweite, d.h. die Konvergenz gegen die semidiskrete Lösung (kontinuierlich in der Zeit, diskret im Ort), wird auf Gitterlevel $L=3$ mit dem Finite-Elemente-Raumpaar $Q_{2} Q_{1}$ untersucht. Dass der Gesamtdiskretisierungsfehler schnell durch den räumlichen Diskretisierungsfehler dominiert wird, hat keinen Einfluss auf die Beobachtungen. Die Maxima werden nur zu vollen Zeitschritten ausgewertet. Bei großen Zeitschritten wird die Wirbelablösung global falsch vorhergesagt, wie am zeitlichen Verlauf des Auftriebskoeffizienten in den Abbildungen 6.4 a) bis d) zu sehen ist. Dementsprechend wenig aussagekräftig sind die Werte für $c_{\mathrm{a}, \max }$ in diesen 

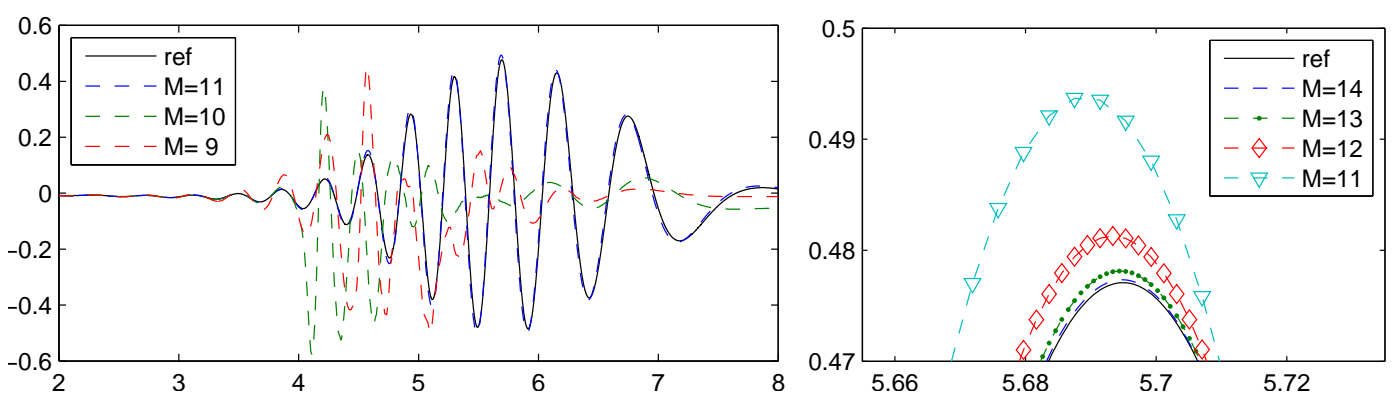

(a) sBDF-Verfahren
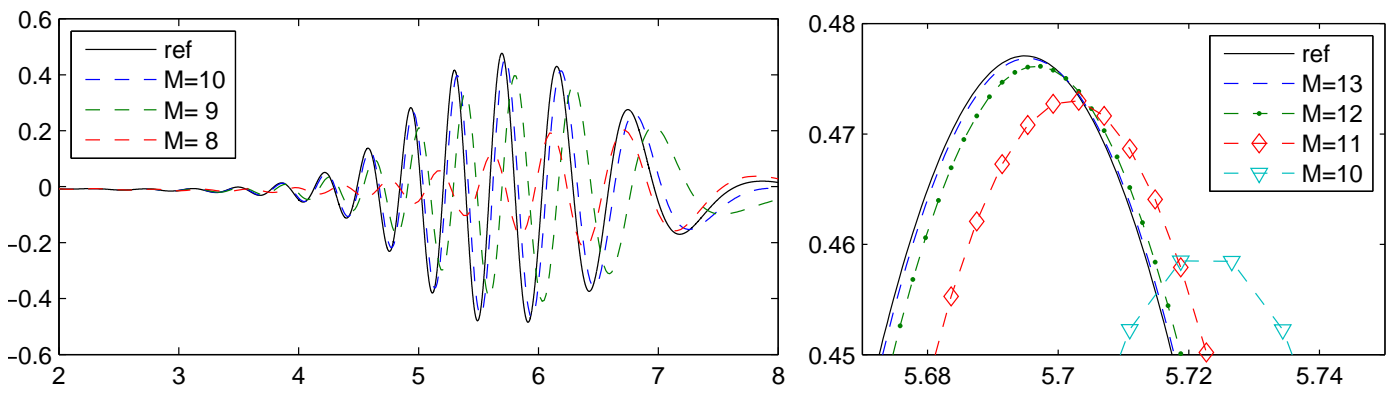

(b) BDF-Verfahren
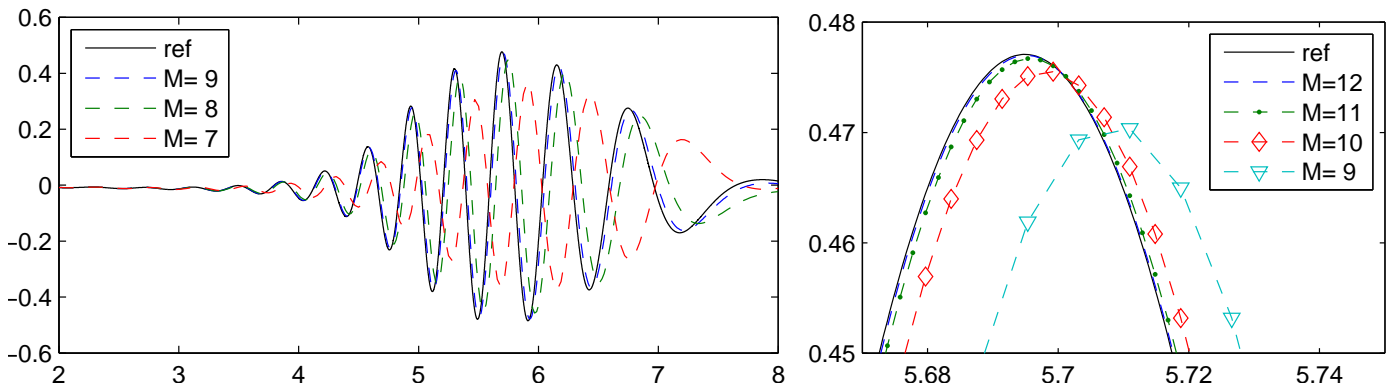

(c) DIRK-Verfahren
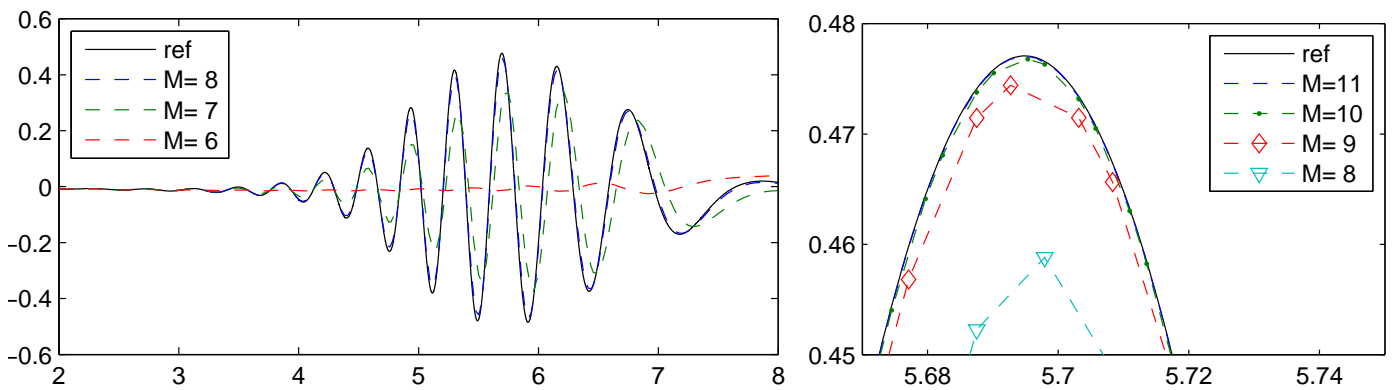

(d) Radau-IIA-Verfahren

Abbildung 6.4: Vergleich der Auftriebsbeiwerte $c_{\mathrm{a}}$ für verschiedene Verfahren und Zeitschrittweiten mit Referenzlösung (links) mit vergrößertem Ausschnitt um das Maximum (rechts). Legende: $M:=\log _{2}(T / \tau)$. 


\begin{tabular}{lrrlll}
\hline & $T / \tau$ & \#lp & $c_{\mathrm{w}, \max }$ & $c_{\mathrm{a}, \max }$ & $\Delta p(8)$ \\
\hline sBDF & 2048 & $2048 / 1.00$ & 2.944552105 & 0.4936839879 & -0.1113675921 \\
& 4096 & $4096 / 1.00$ & 2.944451416 & 0.4812880876 & -0.1117227557 \\
& 8192 & $8192 / 1.00$ & 2.944427979 & 0.4781324024 & -0.1117993011 \\
16384 & $16384 / 1.00$ & 2.944422182 & 0.4773453291 & -0.1118177182 \\
32768 & $32768 / 1.00$ & 2.944420732 & 0.4771484725 & -0.1118223071 \\
\hline BDF & 256 & $2603 / 10.2$ & 2.943763730 & 0.2027777994 & -0.1097358407 \\
& 512 & $4405 / 8.60$ & 2.944099776 & 0.3967787086 & -0.0994915458 \\
& 1024 & $6701 / 6.54$ & 2.944329166 & 0.4585003091 & -0.1110548892 \\
& 2048 & $10029 / 4.90$ & 2.944397744 & 0.4730042316 & -0.1120587787 \\
4096 & $14927 / 3.64$ & 2.944415011 & 0.4761351902 & -0.1119078032 \\
& 8192 & $24108 / 2.94$ & 2.944418957 & 0.4768616448 & -0.1118462758 \\
16384 & $41033 / 2.50$ & 2.944419944 & 0.4770273081 & -0.1118295321 \\
\hline DIRK & 128 & $2904 / 22.7$ & 2.942809235 & 0.3404863133 & -0.1115203290 \\
& 256 & $4720 / 18.4$ & 2.943676958 & 0.4499528780 & -0.1070532845 \\
& 512 & $7319 / 14.3$ & 2.944082332 & 0.4693408067 & -0.1120247398 \\
& 1024 & $11182 / 10.9$ & 2.944261245 & 0.4751071586 & -0.1119716183 \\
2048 & $17138 / 8.37$ & 2.944343168 & 0.4767043146 & -0.1118665299 \\
4096 & $27696 / 6.76$ & 2.944382631 & 0.4769894102 & -0.1118348562 \\
& 8192 & $46800 / 5.71$ & 2.944401642 & 0.4770529992 & -0.1118266154 \\
16384 & $85808 / 5.24$ & 2.944411014 & 0.4770770693 & -0.1118245368 \\
\hline Radau-IIA & 64 & $1694 / 26.5$ & 2.942437979 & 0.0389797873 & -0.1217070769 \\
128 & $3302 / 25.8$ & 2.943960869 & 0.3427873237 & -0.1095877425 \\
& 256 & $5816 / 22.7$ & 2.944336167 & 0.4522718308 & -0.1120145223 \\
512 & $9896 / 19.3$ & 2.944408860 & 0.4714768734 & -0.1118509988 \\
& 1024 & $16278 / 15.9$ & 2.944418262 & 0.4767861814 & -0.1118271131 \\
2048 & $27000 / 13.2$ & 2.944419440 & 0.4770376477 & -0.1118242128 \\
4096 & $46162 / 11.3$ & 2.944420104 & 0.4770691750 & -0.1118238651 \\
& 8192 & $76122 / 9.29$ & 2.944420197 & 0.4770724760 & -0.1118239305 \\
16384 & $121760 / 7.43$ & 2.944420242 & 0.4770823424 & -0.1118239517 \\
\hline & & & & \\
& & & & \\
& & &
\end{tabular}

Tabelle 6.3: Zeitschrittweitenuntersuchung für verschiedene Verfahren auf Gitterlevel $L=3$ mit $Q_{2} Q_{1}$ Elementen. 

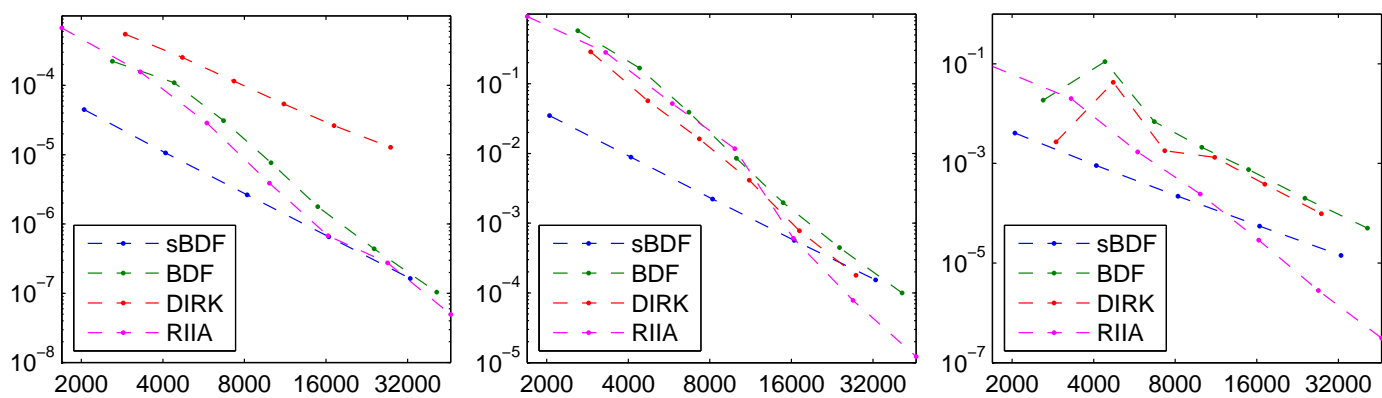

Abbildung 6.5: Relative Fehler der berechneten Kenngrößen gegenüber der Anzahl gelöster linearer Probleme für den Widerstandsbeiwert (links), den Auftriebsbeiwert (mitte) und den Druckabfall (rechts).

Fällen. Für kleine Zeitschrittweiten konvergieren alle untersuchten Verfahren. Im Folgenden werden die Lösungen mit einer Referenzlösung für Gitterlevel $L=3$ verglichen, die mit dem Radau-IIA-Verfahren, $M=13$ und einem Zielresiduum von $10^{-10}$ berechnet wurden.

Das sBDF-Verfahren, mit nur einem Linearisierungsschritt, liefert erst ab $M=11$ brauchbare Ergebnisse, ist aber auch für größere Zeitschrittweiten zumindest noch stabil. Eine sehr gute Übereinstimmung ist erst ab $M=14$ zu beobachten.

Das implizite BDF-Verfahren produziert ab $M=9$ die richtige Anzahl an Wirbeln. Die Ablösung ist jedoch zeitlich etwas verzögert und der Auftriebsbeiwert zu klein. In der Vergößerung ist zu sehen, wie der Phasenfehler bei kleinerer Zeitschrittweite abnimmt. $\mathrm{Ab} M=13$ ist auch in der Vergößerung die Übereinstimmung sehr gut.

Das DIRK-Verfahren liefert ab $M=8$ brauchbare und ab $M=11$ sehr gute Ergebnisse. Es ist deutlich weniger dissipativ als das BDF-Verfahren. Da aber alle Kenngrößen auch vom Druck abhängen, leiden sie unter dem Effekt der Ordnungsreduktion für den Druck.

Das Radau-IIA-Verfahren liefert schon bei $M=7$ eine qualitativ gute Vorhersage der Wirbelablösung mit geringem Phasenfehler. Lediglich die Dissipation ist bei dieser Zeitschrittweite noch zu groß. Für $M=10$ ist die Übereinstimmung bereits sehr gut. Schon ab $M=12$ beginnt der Linearisierungsfehler den Gesamtfehler zu dominieren.

Bei allen impliziten Verfahren ist zu beobachten, dass die Anzahl der benötigten Fixpunktiterationen bei kleinerer Zeitschrittweite deutlich zurückgeht. Das liegt zum einen daran, dass die extrapolierten Startwerte für kleine Zeitschrittweiten besser werden und zum anderen daran, dass die Massematrix im nichtlinearen Problem mit $\tau^{-1}$ multipliziert wird und somit gegenüber dem nichtlinearen Konvektionsterm immer mehr dominiert. Eine Halbierung der Zeitschrittweite erhöht den Aufwand impliziter Verfahren nur um $50 \%$ bis $70 \%$, während sich der Aufwand des linearen sBDF-Verfahrens verdoppelt.

Gemessen an der Zeitschrittweite schneiden die impliziten Verfahren, insbesondere das Radau-IIA-Verfahren, am besten ab. Sie erlauben größere Zeitschritte und sind bei glei- 

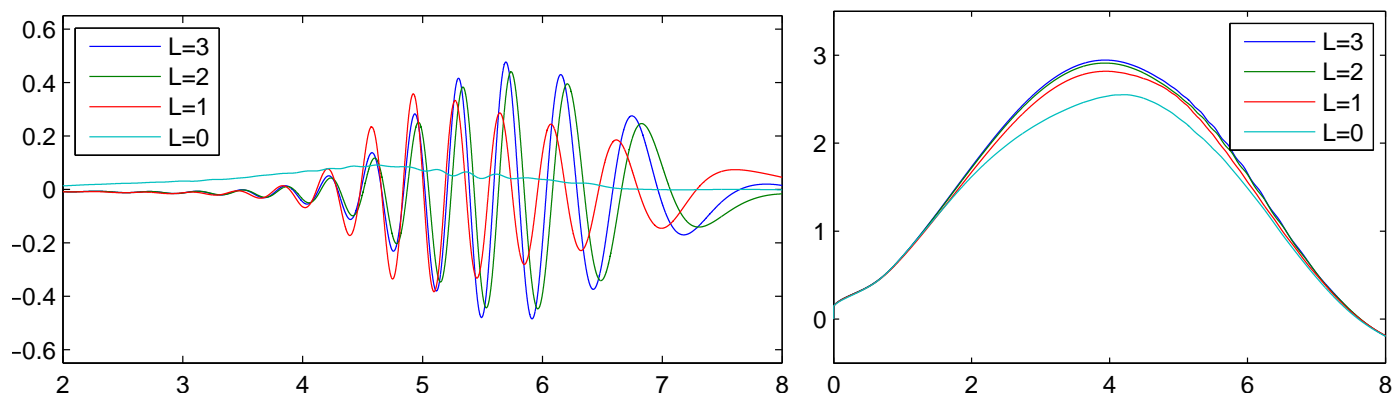

Abbildung 6.6: Gitterkonvergenz für den Auftriebsbeiwert (links) und den Widerstandsbeiwert (rechts).

cher Zeitschrittweite genauer als das linearisierte sBDF-Verfahren. Betrachtet man die Genauigkeit relativ zur Anzahl der gelösten linearen Probleme, ergibt sich jedoch ein anderes Bild. In Abbildung 6.5 ist der relative Fehler der Kenngrößen gegen die Anzahl der gelösten linearen Probleme aufgetragen. Bei geringem Aufwand liefert immer das sBDF-Verfahren die besten Werte, da es deutlich kleinere Zeitschritte machen kann. Wegen der zunehmenden Effizienz der impliziten Verfahren bei kleineren Zeitschrittweiten können diese jedoch teilweise aufholen. Von den impliziten Verfahren ist immer das Radau-IIA-Verfahren am effizientesten.

\subsection{Konvergenzuntersuchung Ort}

Um bei der räumlichen Gitterkonvergenzuntersuchung den Einfluss der Zeitdiskretisierung auszublenden, werden wir das bezüglich der Zeit genaueste Radau-IIA-Verfahren mit 8192 Zeitschritten und einem nicht-linearen Residuum von $10^{-10}$ verwenden. So wird sichergestellt, dass immer der räumliche Diskretisierungsfehler dominiert.

Die Gitterfeinheit hat nur geringen Einfluss auf die Konvergenz der Fixpunktiteration, die Anzahl der Linearisierungsschritte pro Zeitschritt fällt für feinere Gitter nur leicht, aber monoton, ab (siehe Tabelle 6.4).

Auf den groben Gittern wird die Wirbelablösung nicht richtig simuliert. Das Ausgangsgitter ist zu grob, um bei einer Diskretisierung mit $\mathcal{Q}_{2} / \mathcal{Q}_{1}$-Elementen die Wirbelablösung aufzulösen. Auf Gitterlevel $L=1$ findet die Wirbelablösung mit der richtigen Frequenz statt, der Auftriebsbeiwert wird jedoch um bis zu 50\% falsch vorhergesagt (siehe Abbildung 6.6). Erst ab $L=2$ sind die Fehler im einstelligen Prozentbereich. Bei der Verwendung von $\mathcal{Q}_{2} / \mathcal{Q}_{1}$-Elementen liefern schon die Rechnungen auf dem Ausgangsgitter (ohne Verfeinerung) brauchbare Resultate mit relativen Fehlern im einstelligen Prozentbereich.

Das Ausgangsgitter war bisher noch nicht besonders an das Problem angepasst. Da der räumliche Diskretisierungsfehler deutlich größer als der zeitliche Diskretisierungs- 


\begin{tabular}{lcclll}
\hline Element & $L$ & $\# \mathrm{lp}$ & $c_{\mathrm{w}, \max }$ & $c_{\mathrm{a}, \max }$ & $\Delta p(8)$ \\
\hline $\mathcal{Q}_{2} / \mathcal{Q}_{1}$ & 0 & $114720 / 14.0$ & 2.550368290 & 0.0913466573 & -0.1301897322 \\
& 1 & $130348 / 15.9$ & 2.816950918 & 0.3579816784 & -0.1078912593 \\
& 2 & $123452 / 15.1$ & 2.910386745 & 0.4405812236 & -0.1092582103 \\
& 3 & $118364 / 14.5$ & 2.944420202 & 0.4770727570 & -0.1118238501 \\
& 4 & $112952 / 13.8$ & 2.949899411 & 0.4777103109 & -0.1116394501 \\
\hline $\mathcal{Q}_{3} / \mathcal{Q}_{2}$ & 0 & $134574 / 16.4$ & 2.818326730 & 0.4378592593 & -0.1120410124 \\
& 1 & $125618 / 15.3$ & 2.947360241 & 0.4412185448 & -0.1102887913 \\
& 2 & $120674 / 14.7$ & 2.953377386 & 0.4778802122 & -0.1116436756 \\
& 3 & $114850 / 14.2$ & 2.952151395 & 0.4778663931 & -0.1116159627 \\
\hline Referenzwerte & & 2.9509215 & 0.477885 & -0.11162 \\
\hline \multicolumn{6}{l}{} \\
\end{tabular}

Tabelle 6.4: Gitterkonvergenz für $\mathcal{Q}_{2} / \mathcal{Q}_{1}$ - und $\mathcal{Q}_{3} / \mathcal{Q}_{2}$-Elemente.

fehler ist, werden für die folgenden Rechnungen zur Bestimmung von Benchmarkwerten daher besser an die Lösung angepasste Gitter verwendet. Das noch nicht verfeinerte Ausgangsgitter mit $L=0$ wird zum Vergleich mit dem bisherigen Gitter in Abbildung 6.7 dargestellt. Es hat im Bereich um den Zylinder etwa die doppelte Aufösung und ist lokal direkt hinter dem Zylinder feiner als am Ende des Kanals. Auf eine anisotrope Verfeinerung zur Auflösung von Grenzschichten wurde verzichtet, es handelt sich weiterhin um ein isotropes Gitter mit stärker variierender Gitterweite $h$.

Die Resultate in Tabelle 6.5 zeigen, dass durch ein angepasstes Gitter eine deutlich höhere Genauigkeit erreicht werden kann. Die Genauigkeit der Rechnungen auf dem BenchmarkGitter entspricht etwa der Genauigkeit auf dem vorherigen Gitter mit einer zusätzlichen globalen Verfeinerung und passt gut zu der Beobachtung, dass das Benchmark-Gitter im Bereich des Zylinders etwa die doppelte Auflösung hat. Die Auflösung im übrigen Gebiet ist ansonsten vergleichbar oder sogar gröber, was jedoch keinen großen Einfluss
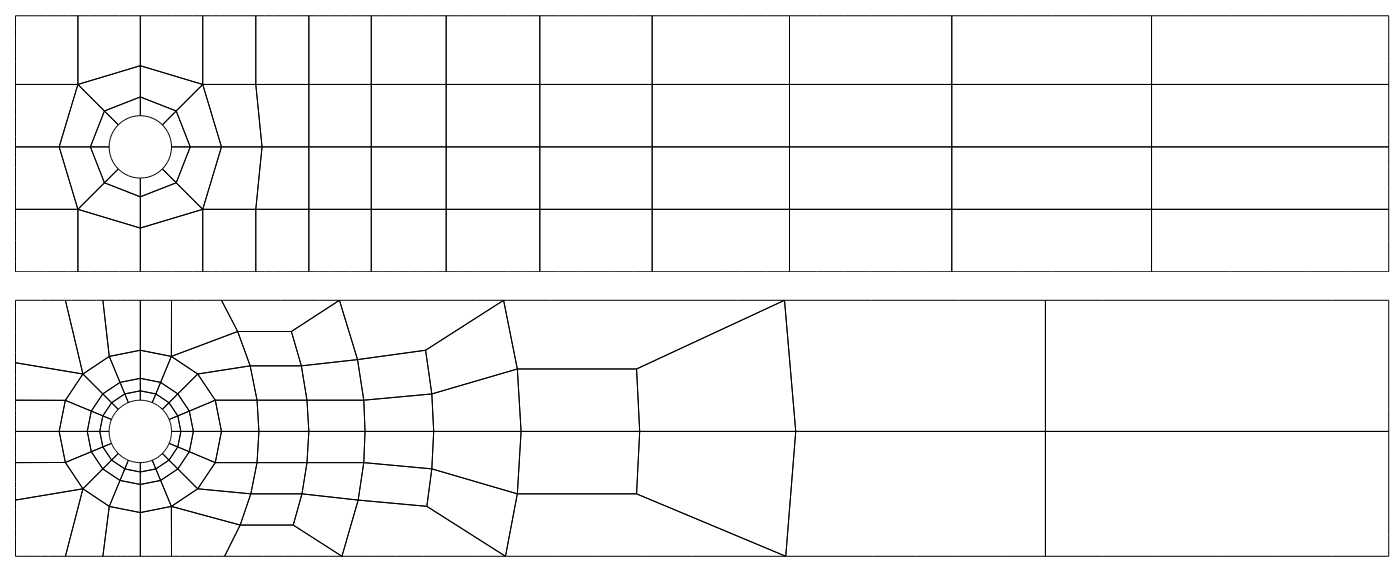

Abbildung 6.7: oben: bisheriges Gitter, unten: Benchmark-Gitter. 
Kapitel 6: Umströmung eines zweidimensionalen Zylinders

\begin{tabular}{lrrrlll}
\hline Element & $L$ & NNZ/1e3 & Zeit pro LP & $c_{\mathrm{w}, \max }$ & $c_{\mathrm{a}, \max }$ & $\Delta p(8)$ \\
\hline $\mathcal{Q}_{2} / \mathcal{Q}_{1}$ & 0 & 17 & 0.04 & 2.551632300 & 0.5274251517 & -0.1143420517 \\
& 1 & 75 & 0.15 & 2.883372765 & 0.5204481478 & -0.1103237793 \\
& 2 & 318 & 0.64 & 2.940557175 & 0.4829426236 & -0.1120909795 \\
& 3 & 1303 & 3.48 & 2.949408583 & 0.4781928947 & -0.1116862080 \\
& 4 & 5276 & 17.6 & 2.950659461 & 0.4778617170 & -0.1116263221 \\
\hline $\mathcal{Q}_{3} / \mathcal{Q}_{2}$ & 0 & 80 & 0.12 & 2.940823438 & 0.5574437381 & -0.1082831163 \\
& 1 & 343 & 0.48 & 2.953440142 & 0.4822723882 & -0.1119131591 \\
& 2 & 1423 & 2.24 & 2.952200466 & 0.4779863575 & -0.1116527497 \\
& 3 & 5794 & 11.0 & 2.951270283 & 0.4778910855 & -0.1116241051 \\
\hline $\mathcal{Q}_{4} / \mathcal{Q}_{3}$ & 0 & 232 & 0.31 & 2.960731583 & 0.4879541344 & -0.1119175676 \\
& 1 & 985 & 1.22 & 2.953478865 & 0.4779589292 & -0.1116511281 \\
& 2 & 4055 & 5.92 & 2.951346384 & 0.4778988674 & -0.1116237287 \\
\hline \multicolumn{2}{l}{ Referenzwerte } & & & 2.950921 & 0.477885 & -0.11162 \\
\hline
\end{tabular}

Tabelle 6.5: Gitterkonvergenz auf dem Bechmark-Gitter.

auf die Genauigkeit hat.

Auf dem Benchmark-Gitter wurden Rechnungen für Finite-Elemente-Räume bis zur Ordnung $k=4$ durchgeführt. Wieder zeigt sich, dass die Genauigkeit durch Erhöhung der Elementordnung stark zunimmt. Gleichzeitig wächst aber auch die Anzahl der nicht verschwindenden Matrixeinträge (NNZ) stark an, da mehr Basisfunktionen innerhalb der Zellen miteinander gekoppelt sind (siehe dritte Spalte in Tabelle 6.5). Die Effizienz des linearen Lösers profitiert jedoch etwas von der zunehmenden Dichtheit der linearen Gleichungssysteme. Diese Entwicklung lässt sich jedoch nicht pauschal auf andere Löser (z.B. iterative Krylov-Verfahren) verallgemeinern.

Insgesamt sind die Resultate hinsichtlich der räumlichen Diskretisierung zufriedenstellend. Auf den feinsten verwendeten Gittern betragen die relativen Fehler für alle untersuchten Kenngrößen etwa 0.01\%. Im Gegensatz dazu sind die Referenzwerte aus [37] für $c_{\mathrm{w}, \max }$ deutlich genauer als für $\Delta p(8)$. Der Vorteil der in der vorliegenden Arbeit verwendeten inf-sup stabilen Elemente liegt in einer stabilen Approximation des Drucks, die eine Druckstabilisierung und die damit verbundene Parameterwahl überflüssig macht. Für die Rechnungen ohne Turbulenzmodell in diesem Kapitel sind keine zusätzlichen Parameter von Anwender zu wählen.

\subsection{Fazit}

Das Hauptziel dieses Kapitels ist die Bewertung der verschiedenen Verfahren zur Zeitdiskretisierung. Insbesondere aus Abbildung 6.5 lässt sich diesbezüglich folgendes Fazit 

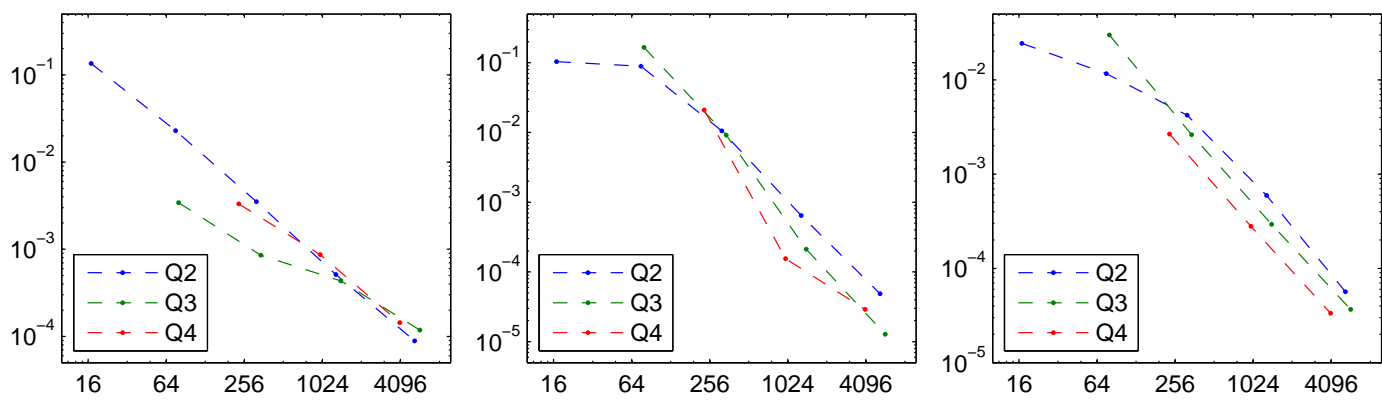

Abbildung 6.8: Relative Fehler von $c_{\mathrm{w}, \max }$ (links), $c_{\mathrm{a}, \max }$ (mitte) und $\Delta p(8)$ (rechts) gegenüber der Anzahl der Matrixeinträge (in tausend) für unterschiedliche Elementordnungen.

ziehen: Die Wahl des am besten geeigneten Verfahren hängt wesentlich vom angestrebten Fehler ab. Für Rechnungen mit sehr hoher zeitlicher Genauigkeit eignen sich am besten Verfahren mit hoher Konvergenzordnung, wie z.B. das Radau-IIA-Verfahren. Der zusätzliche Aufwand für die implizite Kopplung mehrerer Stufen wird dabei durch die höhere Konvergenzordnung ausgeglichen. Auch der Aufwand zur Iteration der NichtLinearität wird mit kleiner werdender Zeitschrittweite geringer.

Wird die Genauigkeit der räumlichen Diskretisierung berücksichtigt und die Anforderung an die Genauigkeit der zeitlichen Diskretisierung daran angepasst, so liefert bei vergleichbarem Aufwand immer das sBDF-Verfahren die besten Ergebnisse. Gerade auch für turbulente Strömungen, bei denen keine hohe Genauigkeit in der räumlichen Diskretisierung erreicht werden kann und gleichzeitig die Lösungen zeitlich stark fluktuieren können, eignen sich die sBDF-Verfahren besonders. Diese erlauben bei vergleichbarem Gesamtaufwand den kleinsten Zeitschritt aller Verfahren. Während der Berechnung von stationären Lösungen eine spezielle Rolle zukommt (siehe Kapitel 7), wird daher für die Berechnung turbulenter Strömungen in Kapitel 8 das sBDF-Verfahren zweiter Ordnung verwendet. 


\section{Kapitel 7}

\section{Laminare nicht-isotherme Hohlraumströmung}

In diesem Kapitel sollen numerische Resultate zur laminaren nicht-isothermen Hohlraumströmung präsentiert werden. Es handelt sich um ein Problem freier Konvektion, bei der die Strömung nur von den durch Temperaturunterschiede hervorgerufenen Auftriebseffekten angetrieben wird. Im Gegensatz zum Benchmarkproblem des vorherigen Kapitels wird jetzt das vollständige Oberbeck-Boussinesq-Modell verwendet. Die nichtisotherme Hohlraumströmung kann als Prototyp für eine Raumluftströmung angesehen werden, bei der die Strömung ebenfalls ausschließlich von unterschiedlich warmen Oberflächen (z.B. Heizkörpern oder Kühlelementen) angetrieben wird. Eine zentrale Rolle spielt dabei der Wärmetransfer zwischen heißen bzw. kalten Oberflächen und dem Fluid. Wichtig für die thermische Behaglichkeit ist auch die Strömungsgeschwindigkeit. Zunächst beschränken wir uns auf den eher akademischen Fall kleiner Rayleigh-Zahlen, für den sich stationäre laminare Strömungen einstellen. Eine turbulente Hohlraumströmung wird im nächsten Kapitel untersucht.

\subsection{Problembeschreibung}

Beim Gebiet $\Omega=[0,1]^{2} \subset \mathbb{R}^{2}$ handelt es sich um das zweidimensionale Einheitsquadrat. Für das Geschwindigkeitsfeld u schreiben wir Haftbedingungen an allen Wänden vor. An den horizontalen Wänden oben und unten verwenden wir isolierende Randbedingungen für die Temperatur $\theta$, während an den vertikalen Wänden links und rechts die Temperatur durch $\theta_{\text {warm }}=0,5$ und $\theta_{\text {kalt }}=-0,5$ festgelegt wird. Dabei handelt es sich um eine skalierte Abweichung von einer mittleren Temperatur. Die Gravitationsbeschleunigung wirkt senkrecht nach unten. Für die Prantl-Zahl wird etwa der Wert $\operatorname{Pr}=0,71$ von Luft bei Raumtemperatur verwendet. Durch den Temperaturunterschied steigt das Fluid an der warmen Wand nach oben und sinkt an der kalten Wand wieder 

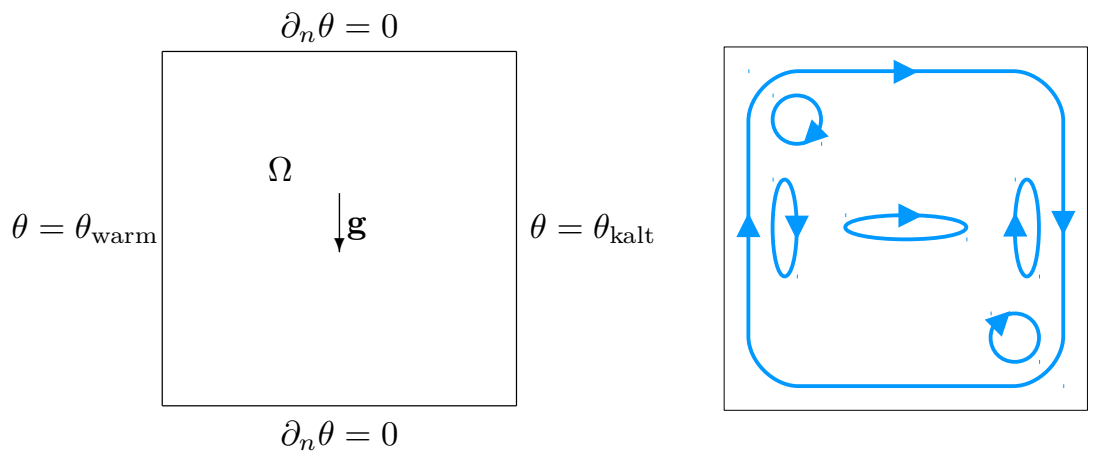

Abbildung 7.1: Darstellung des Gebiets $\Omega$ mit Randbedingungen und Skizze möglicher Rezirkulationszonen für große Rayleigh-Zahlen.

nach unten. Abhängig von der Rayleigh-Zahl entstehen eine große Zirkulation entlang des Gebietsrandes und weitere Rezirkulationszonen im Inneren des Gebiets. Eine schematische Darstellung der Geometrie und der Rezirkulationszonen ist in Abbildung 7.1 skizziert. Als Gleichungsmodell für $\mathbf{u}, p$ und $\theta$ verwenden wir wie bereits erwähnt das Oberbeck-Boussinesq-Modell (3.33) bis (3.35).

Im Folgenden beschränken wir uns auf den Bereich Ra $\leq 10^{7}$, für den die Lösungen laminar sind und für $t \rightarrow \infty$ gegen einen stationären Grenzwert konvergieren. Ab der kritischen Rayleigh-Zahl $\operatorname{Ra}_{\text {crit }}=(1,82 \pm 0,01) \times 10^{8}$ (siehe Ref. [45]), bei der die erste Bifurkation auftritt, werden die Lösungen instationär und für noch größere RayleighZahlen auch turbulent. Diesen Fall (mit etwas anderen Randbedingungen) werden wir im nächsten Kapitel betrachten. Zur Skalierung der Lösung werden folgende Referenzgrößen verwendet:

$$
L_{r}=H, \quad U_{r}=\frac{\alpha}{H \mathrm{Ra}^{1 / 2}}, \quad t_{r}=\frac{L_{r}}{U_{r}}=\frac{H^{2} \mathrm{Ra}^{1 / 2}}{\alpha}, \quad \Delta T=T_{h}-T_{c} .
$$

Diese sind für freie Konvektion an einer vertikalen beheizten Platte bemessen.

\subsection{Beschreibung der Benchmarkwerte}

Wie beim vorherigen Testproblem aus Kapitel 6, ist die exakte Lösung für die Hohlraumströmung mit differentiell beheizten Seitenwänden nicht bekannt. Zur Auswertung der numerischen Lösungen werden daher spezielle Kenngrößen herangezogen. Da für kleine Rayleigh-Zahlen keine experimentellen Daten vorliegen, werden wir die Ergebnisse ausschließlich mit Resultaten anderer numerischer Untersuchungen des Problems vergleichen.

Neben einer qualitativen Betrachtung des Geschwindigkeitsfeldes, bei der die Lage verschiedener Rezirkulationszonen untersucht wird, werden insbesondere Geschwindig- 
keitsprofile auf der vertikalen und horizontalen Mittellinie der Kammer gezeigt. Tabellarisch erfasst werden die Extrema der $\mathrm{u}$-/v-Komponente der Geschwindigkeit $u_{\max }, v_{\max }$ auf den beiden Querschnitten.

Für thermische Strömungen spielt der lokale horizontale Wärmestrom

$$
q(x, y)=u \theta-\alpha \frac{\partial \theta}{\partial x}
$$

eine wichtige Rolle. Der totale Wärmestrom durch die vertikale Ebene mit fester $x$ Koordinate ist gegeben durch:

$$
q(x)=\int_{0}^{1} q(x, s) d s
$$

Für isolierte horizontale Wände und stationäre Lösungen ist dieser unabhängig von der $x$-Koordinate. Wir werden zur Überprüfung $q$ auf zwei senkrechten Querschnitten berechnen, einmal an der heißen Wand $q_{0}:=q(0)$ und einmal in der Gebietsmitte $q_{\text {mid }}:=q(0,5)$. Außerdem werden wir $q$ einmal über das gesamte Gebiet $\Omega$ mitteln. Da es sich um Finite-Elemente-Lösungen handelt, lassen sich diese Integrale mittels numerischer Quadraturformeln exakt auswerten. Aus dem horizontalen Wärmestrom lässt sich die dimensionslose Nusselt-Zahl berechnen, die das Verhältnis von konvektivem Wärmetransport $h$ zu diffusivem Wärmetransport bei ruhendem Fluid beschreibt:

$$
\mathrm{Nu}=\frac{h L}{\alpha}, \quad h=\frac{q}{A \Delta T} .
$$

In unserem entdimensionalisierten Beispiel sind $L=A=\Delta T=1$ und somit $\mathrm{Nu}=\frac{q}{\alpha}$. So erhalten wir entsprechend drei Nusselt-Zahlen $\mathrm{Nu}_{0}, \mathrm{Nu}_{\text {mid }}, \mathrm{Nu}_{\text {avg }}$, abhängig davon, wie $q$ berechnet wurde. Zusätzlich werden wir die lokalen Extrema $\mathrm{Nu}_{\min }$ und $\mathrm{Nu}_{\max }$ der Nusselt-Zahl an der heißen Wand untersuchen.

Bemerkung 7.1 Für die exakte Lösung gilt $\mathrm{Nu}_{0}=\mathrm{Nu}_{\text {mid }}=\mathrm{Nu}_{\text {avg }}$, wir werden jedoch sehen, dass die Berechnungen für diskrete Lösungen unterschiedlich genau sind. Für $x=0$ ist auch $u=0$ und somit $q_{0}$ allein vom Temperaturgradienten in der Grenzschicht abhängig. Daher wird die Genauigkeit von $\mathrm{Nu}_{0}$ wesentlich von der Auflösung der Wandgrenzschichten abhängen. Für $\mathrm{Nu}_{\text {avg }}$ können wir hingegen ausnutzen, dass

$$
\int_{0}^{1} \frac{\partial \theta_{h}(x, y)}{\partial x} \mathrm{~d} x=\theta_{h}(1, y)-\theta_{h}(0, y)=\theta_{\text {kalt }}-\theta_{\text {warm }}
$$

ist. $\mathrm{Nu}_{\text {avg }}$ hängt also nicht von Ableitungen der Lösung ab. Daher können wir für $\mathrm{Nu}_{\text {avg }}$ die höchste Genauigkeit erwarten.

Zur Untersuchung von zweidimensionalen Strömungen lässt sich außerdem die Stromfunktion $\Phi$ heranziehen. Diese ist durch $\nabla \Phi=\mathbf{u}^{\perp}$ definiert, d.h., die Stromlinien des Geschwindigkeitsfeldes entsprechen den Isolinien der Stromfunktion. Diese Vorgehensweise hat den Vorteil, dass sich durch die Isolinien von $\Phi$ automatisch geschlossene 
Stromlinien ergeben. Bei einer numerischen Integration des Geschwindigkeitsfeldes ist dies in der Regel nicht der Fall ist. Numerisch lässt sich die Stromfunktion durch Lösen des Poisson-Problems

$$
\nabla \cdot(\nabla \Phi)=\nabla \cdot \mathbf{u}^{\perp} \text { in } \Omega, \quad \Phi=0 \text { auf } \partial \Omega
$$

bestimmen. Die Randbedingung ergibt sich daraus, dass die Tangentialableitung $\mathbf{n}^{\perp} \cdot \nabla \Phi=\mathbf{n} \cdot \mathbf{u}=0$ auf dem ganzen Rand $\partial \Omega$ verschwindet und wir somit eine beliebige Konstante vorschreiben können.

Dieses Hilfsproblem wird auf dem gleichen Gitter wie das Strömungsproblem mittels einer Finite-Elemente-Methode höherer Ordnung gelöst, um weitere Diskretisierungsfehler zu minimieren. Lokale Extrema der Stromfunktion beschreiben Wirbelzentren. Wir werden den Wert des Maximums $\Phi_{\max }$ der Stromfunktion im oberen linken Viertel des Gebiets tabellarisch angeben.

Als Referenzwerte für die Benchmarkgrößen verwenden wir Werte aus verschiedenen Veröffentlichungen zu numerischen Untersuchungen des Problems. Ältere Resultate in Ref. [17] von 1983 und Ref. [65] von 1989 basieren auf einer Umformulierung der NavierStokes-Gleichungen mittels der Stromfunktion $\Phi$ und der Vortizität $\omega=\nabla \times \mathbf{u}$. In beiden Arbeiten werden Finite-Differenzen-Schemata zweiter bzw. höherer Ordnung verwendet. Die Gitterauflösung beträgt nur bis zu 80x80 bzw. 50x50 Knoten. In der Arbeit [44] von 1991 wird eine pseudo-spektrale Chebyshev-Methode mit einem Polynomgrad von bis zu 128 in jeder Koordinatenrichtung verwendet. Bei der in Ref. [26] verwendeten Spektrale-Elemente-Methode handelt es sich um eine Finite-Elemente-Methode mit niedriger Gitterauflösung aber hohem Polynomgrad. Für die Entkopplung von Geschwindigkeit und Druck wird dort eine Druck-Korrektur-Methode eingesetzt. Diese Methode ist bezüglich der räumlichen Diskretisierung der in dieser Arbeit verwendeten Methode am ähnlichsten.

\subsection{Zeitabhängiger Lösungsverlauf}

Die laminaren stationären Lösungen berechnen wir durch eine zeitabhängige Simulation, bei deren Start sich das Medium in Ruhe befindet und ein linearer Temperaturverlauf vorliegt. Für diese Startphase liegen keine Referenzwerte zum Vergleich vor. Wir betrachten daher zunächst nur den zeitlichen Verlauf von $\mathrm{Nu}_{0}$ für verschiedene Rayleigh-Zahlen in Abbildung 7.2 (links). Nach einer Einschwingphase konvergiert die Lösung und damit auch die Nusselt-Zahl gegen einen stationären Grenzwert. Für große Rayleigh-Zahlen und die damit verbundene geringe Viskosität dauert diese Einschwingphase länger.

Der rechte Teil der Abbildung zeigt den Verlauf der Nusselt-Zahl für Ra $=10^{6}$ und verschiedene Zeitschrittweiten $\tau$. Kleine $\tau$ liefern zwar einen genaueren zeitlichen Verlauf, ändern aber am stationären Grenzwert nichts. Eine große Zeitschrittweite hat sogar den Vorteil, dass das Verfahren dissipativer ist und schneller konvergiert. Daher wird 

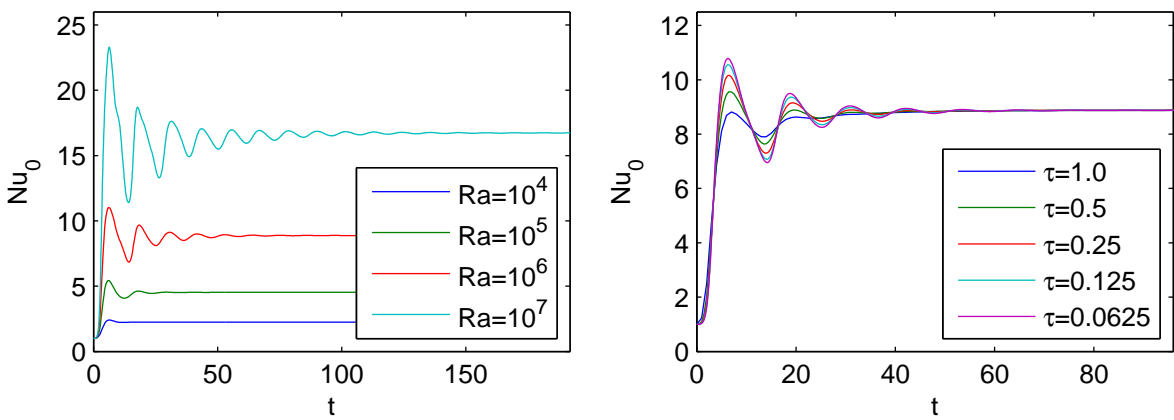

Abbildung 7.2: Zeitabhängiger Verlauf von $\mathrm{Nu}_{0}$ für verschiedene Rayleigh-Zahlen (links) und Zeitschrittweiten des impliziten Euler-Verfahrens mit zwei Fixpunktiterationsschritten für $\mathrm{Ra}=10^{6}$ (rechts).

zur Berechnung der stationären Grenzwerte ein IMEX-Euler Verfahren mit großer Zeitschrittweite $\tau=1$ verwendet. Als Konvergenzkriterium wird $\left\|\partial_{t} \mathbf{u}_{h}\right\|_{0} \leq 10^{-12}$ in der Euklidschen Norm des $\mathbb{R}^{n_{u}}$ verwendet. Diese Vorgehensweise zeigte bessere Konvergenzeigenschaften als eine einfache Fixpunktiteration des stationären Problems und führt zudem durch die Diskretisierung der Zeitableitung zu besseren algebraischen Eigenschaften der zu lösenden linearen Probleme.

\subsection{Beschreibung wesentlicher Strömungsmerkmale der stationären Lösung}

Wir betrachten zunächst die Entwicklung der Lösungen für wachsende Rayleigh-Zahlen, die in Abbildung 7.3 dargestellt ist. Die Grenzschicht der Temperatur $\theta$ an den beheizten und gekühlten Wänden wird für große Rayleigh-Zahlen immer dünner, während sich gleichzeitig ein nahzu linearer vertikaler Temperaturverlauf in der Gebietsmitte einstellt. Für kleine Rayleigh-Zahlen $\mathrm{Ra} \leq 10^{4}$ wird das Geschwindigkeitsfeld durch einen zentralen Hauptwirbel dominiert. Für größere Rayleigh-Zahlen wandern die Bereiche großer Geschwindigkeitsbeträge näher an den Rand des Gebiets und es bilden sich ebenfalls Grenzschichten. Zusätzlich entstehen verschiedene kleine Rezirkulationszonen im Gebiet. Dass die Normierung des Geschwindigkeitsfeldes mittels (7.1) korrekt gewählt wurde, wird dadurch bestätigt, dass sich unabhängig von der Rayleigh-Zahl eine maximale Geschwindigkeit von $\|\mathbf{u}\|_{\infty} \approx 0,22$ einstellt.

Für große Rayleigh-Zahlen Ra $\geq 10^{6}$ kommt das Fluid in einem großen Bereich in der Gebietsmitte fast zum Stillstand. Im Gegensatz dazu bildet sich bei isothermen Hohlraumströmungen mit einer bewegten Seitenwand im Zentrum der Kammer eine Festkörperrotation aus, bei der sich das Fluid auf Kreisbahnen um das Wirbelzentrum bewegt und die Geschwindigkeit proportional zum Abstand zu dem Wirbelzentrum ist [23]. Im nicht-isothermen Fall würde durch einen sich im Uhrzeigersinn drehenden Wir- 

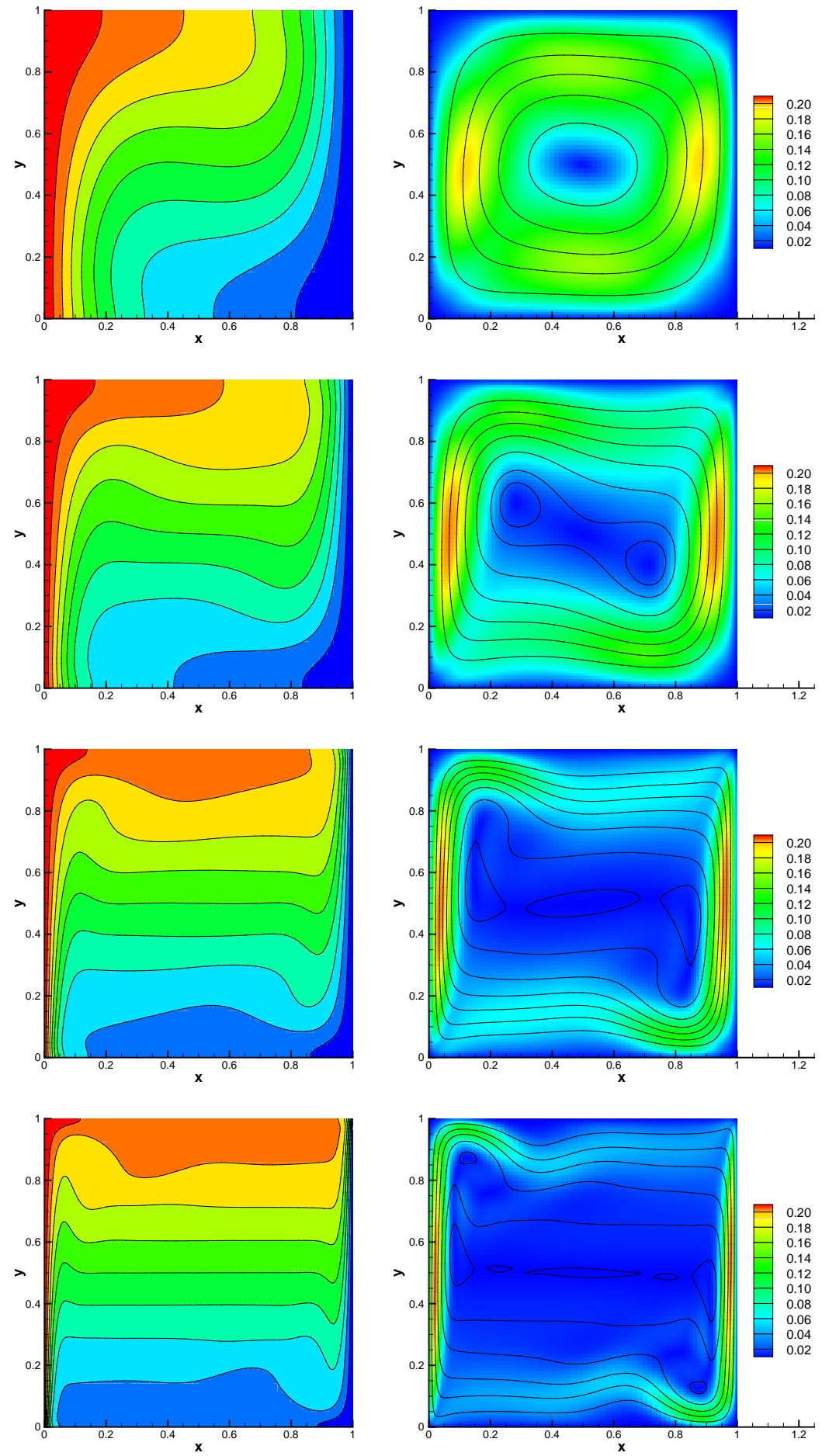

Abbildung 7.3: Temperatur $\theta$ (links) und Geschwindigkeitsbetrag $|\mathbf{u}|$ mit Stromlinien (rechts) für Ra $\in\left\{10^{4}, 10^{5}, 10^{6}, 10^{7}\right\}$ (von oben nach unten). 

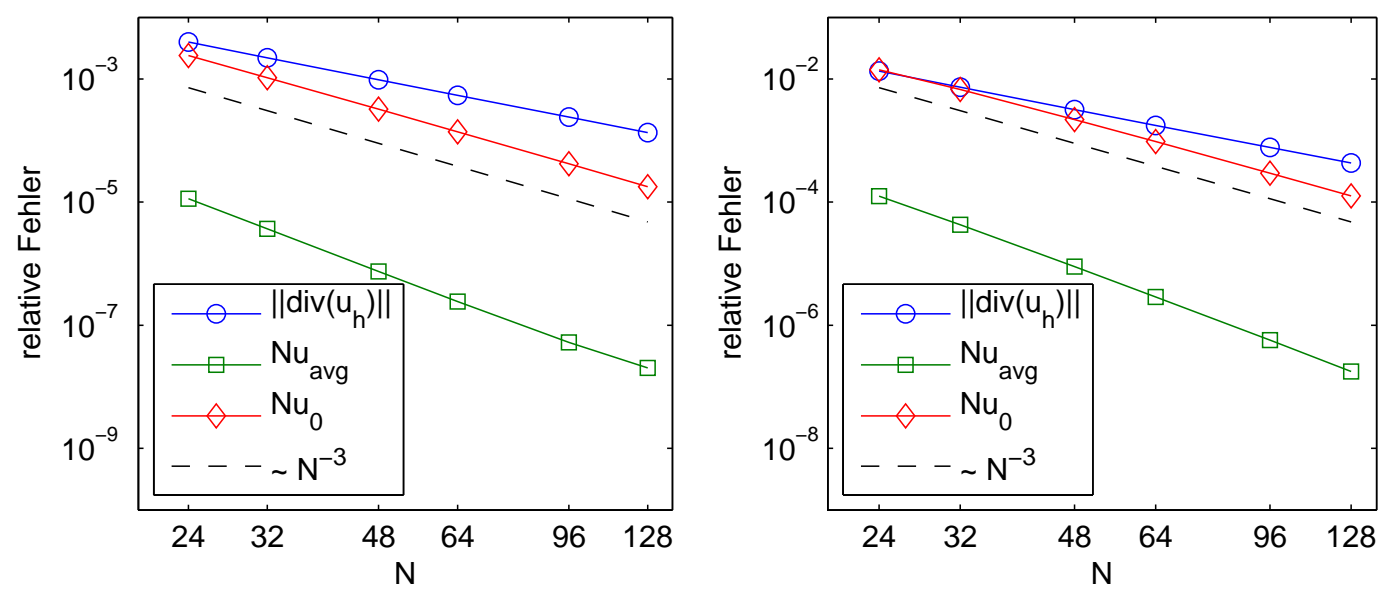

Abbildung 7.4: Konvergenz ausgewählter Größen für Ra $=10^{4}$ (links) und Ra $=10^{5}$ (rechts) auf äquidistanten Gittern mit $\mathcal{Q}_{2} / \mathcal{Q}_{1} / \mathcal{Q}_{2}$-Elementen und $N^{2}$ Zellen.

bel warmes Fluid nach unten und kaltes Fluid nach oben transportiert. Dem wirken jedoch die Auftriebskräfte entgegen. Die vertikale Temperaturschichtung wirkt also stabilisierend auf das Fluid und sorgt dafür, dass abgesehen von den vertikalen Wänden die meisten Stromlinien horizontal verlaufen.

\subsection{Gitterkonvergenzstudie}

Die Untersuchungen in diesem Abschnitt sind in drei Teile gegliedert. Zuerst wird die Konvergenz für das $\mathcal{Q}_{2} / \mathcal{Q}_{1} / \mathcal{Q}_{2}$-Element auf äquidistanten Gittern untersucht. Dann werden zur Verbesserung der Approximation anisotrope Gitter verwendet und zuletzt auch Elemente mit höherer polynomieller Ordnung betrachtet. Alle tabellarischen Ergebnisse sind am Ende dieses Kapitels zu finden.

Die Ergebnisse für das $\mathcal{Q}_{2} / \mathcal{Q}_{1} / \mathcal{Q}_{2}$-Element auf äquidistanten Gittern sind für die Rayleigh-Zahlen Ra $\in\left\{10^{4}, 10^{5}, 10^{6}\right\}$ in Tabelle 7.3 auf Seite 117 zusammen mit Literaturwerten angegeben. Die Auflösung des Gitters ist durch die Anzahl der Zellen $N$ in jede Koordinatenrichtung angegeben. Die Übereinstimmung mit den Referenzwerten ist sehr gut. Für größere Rayleigh-Zahlen nimmt der Diskretisierungsfehler jedoch zu, was auf die Entstehung von Grenzschichten zurückzuführen ist, die durch äquidistante Gitter schlecht aufgelöst werden. Abbildung 7.4 zeigt die Konvergenz ausgewählter Kenngrößen gegen extrapolierte Grenzwerte. Dabei wird eine Konvergenzrate von bis zu $\mathcal{O}\left(N^{-3}\right)$ erreicht. Bemerkenswert ist die hohe relative Genauigkeit von $\mathrm{Nu}_{\mathrm{avg}}$, die für $\mathrm{Ra}=10^{4}$ etwa $10^{-8}$ und für $\mathrm{Ra}=10^{5}$ etwa $10^{-7}$ beträgt.

Um die Grenzschichten für große Rayleigh-Zahlen besser aufzulösen, werden jetzt ani- 

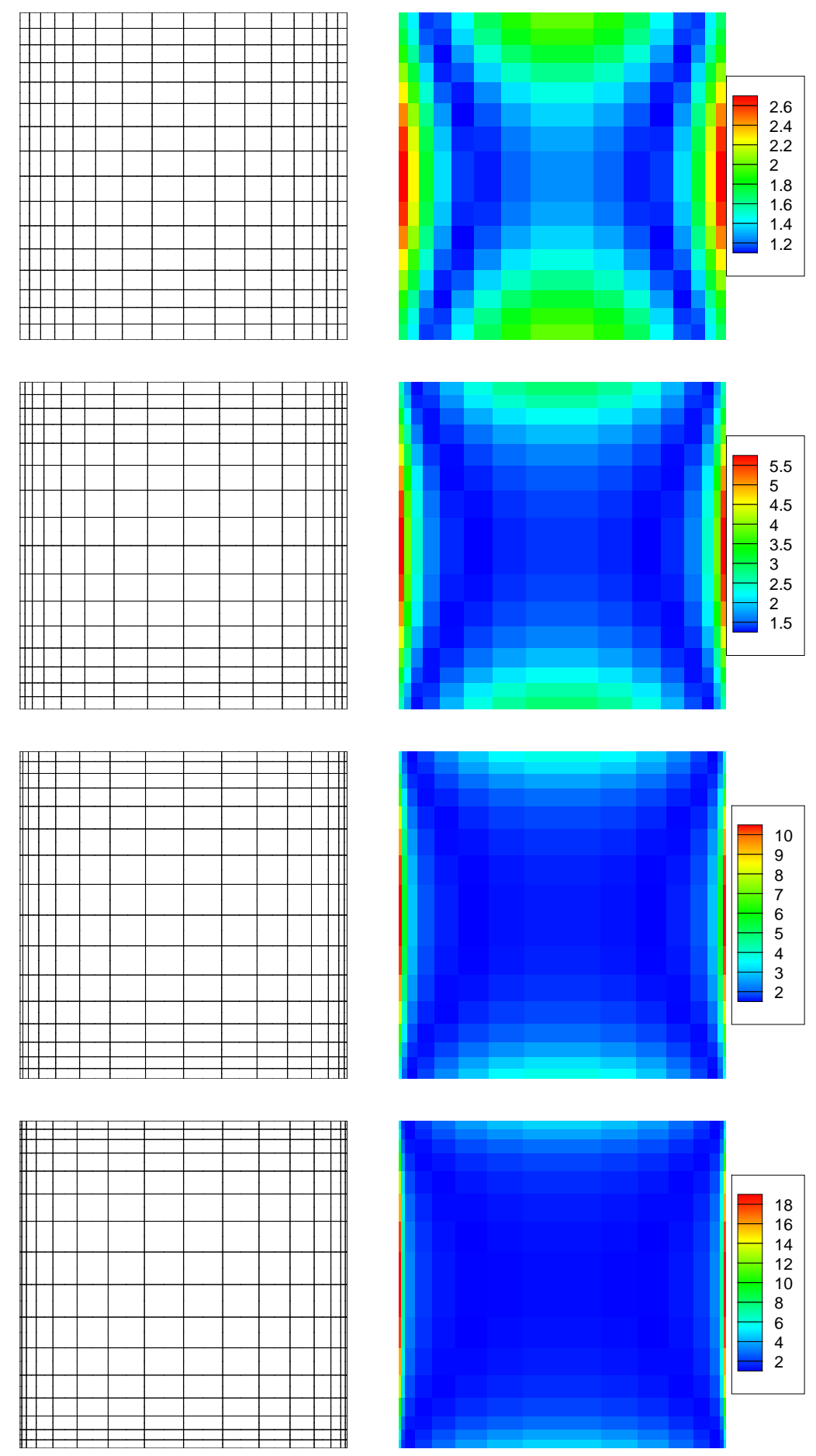

Abbildung 7.5: Angepasste anisotrope Gitter für die Rayleigh-Zahlen Ra $\in\left\{10^{4}, 10^{5}, 10^{6}, 10^{7}\right\}$ (von oben nach unten) und Darstellung des zellweisen Seitenverhältnisses (rechts). 

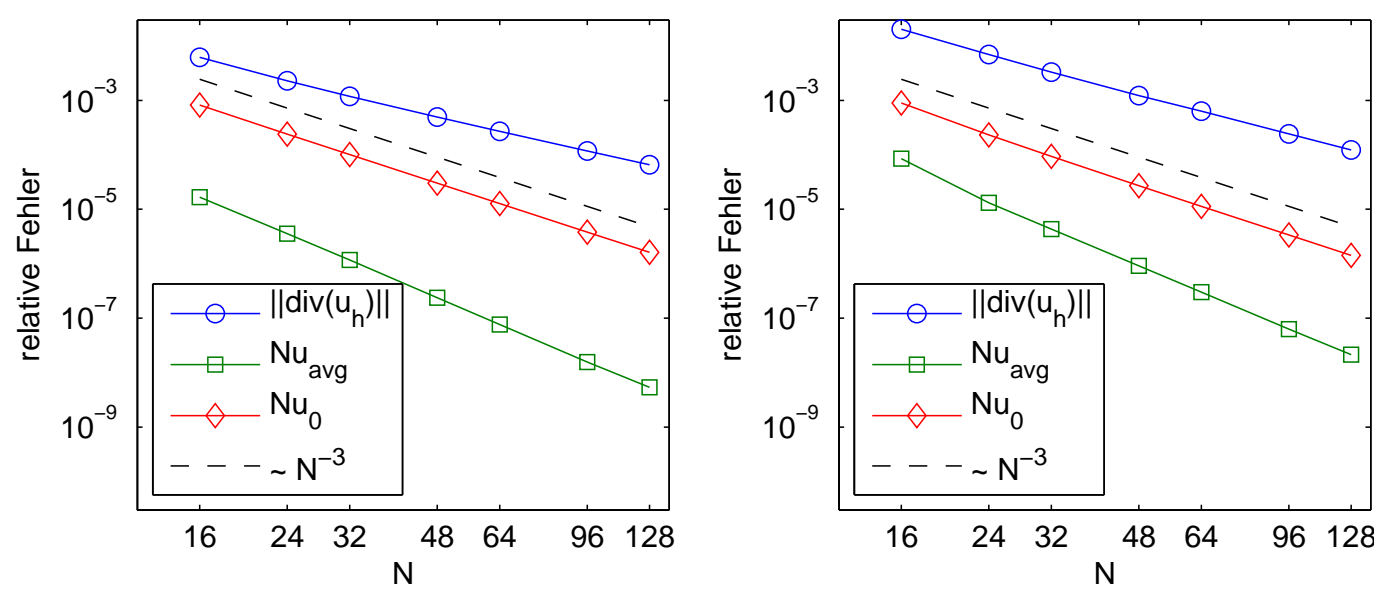

Abbildung 7.6: Konvergenz ausgewählter Größen für Ra $=10^{4}$ (links) und Ra $=10^{5}$ (rechts) auf anisotropen Gittern mit $\mathcal{Q}_{2} / \mathcal{Q}_{1} / \mathcal{Q}_{2}$-Elementen und $N^{2}$ Zellen.

sotrope Gitter verwendet. Dazu transformieren wir die Knoten der äquidistanten Gitter mit Abbildungen $T_{a, b}:[0,1]^{2} \rightarrow[0,1]^{2}$, die die Knoten näher an den Gebietsrand bewegen. Diese Abbildungen sind durch

$$
\left(\begin{array}{l}
x \\
y
\end{array}\right) \mapsto T_{a, b}\left(\left(\begin{array}{l}
x \\
y
\end{array}\right)\right):=\left(\begin{array}{l}
x-(1-a) \frac{1}{2 \pi} \sin (2 \pi x) \\
y-(1-b) \frac{1}{2 \pi} \sin (2 \pi y)
\end{array}\right)
$$

gegeben. Die Parameter $0<a, b \leq 1$ steuern dabei die Streckung der Zellen am Rand des Gebiets. An den vertikalen Wänden ergibt sich ein Seitenverhältnis von etwa $a: 2-b$ während sich an den horizontalen Wänden ein Seitenverhältnis von $b: 2-a$ ergibt. Da die Nusselt-Zahl der mittleren Ableitung der Temperatur an der vertikalen Wand entspricht, verwenden wir $a \approx \mathrm{Nu}^{-1}$ und $b \approx \mathrm{Nu}^{-1 / 3}$. So wird erreicht, dass immer ausreichend viele Zellen innerhalb der Grenzschichten liegen. In der Gebietsmitte entspricht die Auflösung unabhängig von der Streckung mindestens der halben Auflösung der äquidistanten Gitter. Grobe Gitter mit $16^{2}$ Zellen für die entsprechenden RayleighZahlen sind in Abbildung 7.5 dargestellt. Die lokale Ähnlichkeit der Seitenverhältnisse benachbarter Zellen, eine der Voraussetzungen an das Gitter zur Konstruktion eines anisotropen divergenzerhaltenden Interpolationsoperators, ist deutlich zu sehen.

Die Ergebnisse der Rechnungen auf den anisotropen Gittern für Ra $\in\left\{10^{4}, 10^{5}, 10^{6}, 10^{7}\right\}$ sind in Tabelle 7.4 auf Seite 118 aufgelistet. Im Verlgeich zu den uniformen Gittern nimmt die Genauigkeit für $\mathrm{Nu}_{\text {mid }}$ etwas ab, während die Genauigkeit für alle Größen innerhalb der Grenzschicht deutlich zunimmt. Da die Gitter außerdem für jede Rayleigh-Zahl angepasst sind, ist auch der Genauigkeitsverlust für steigende Rayleigh-Zahlen geringer als bei den uniformen Gittern. Wie in Abbildung 7.6 zu sehen ist, bleiben die Konvergenzraten auch auf den anisotropen Gittern erhalten.

Eine weitere Möglichkeit, die Genauigkeit der Approximation zu verbessern, ist die 

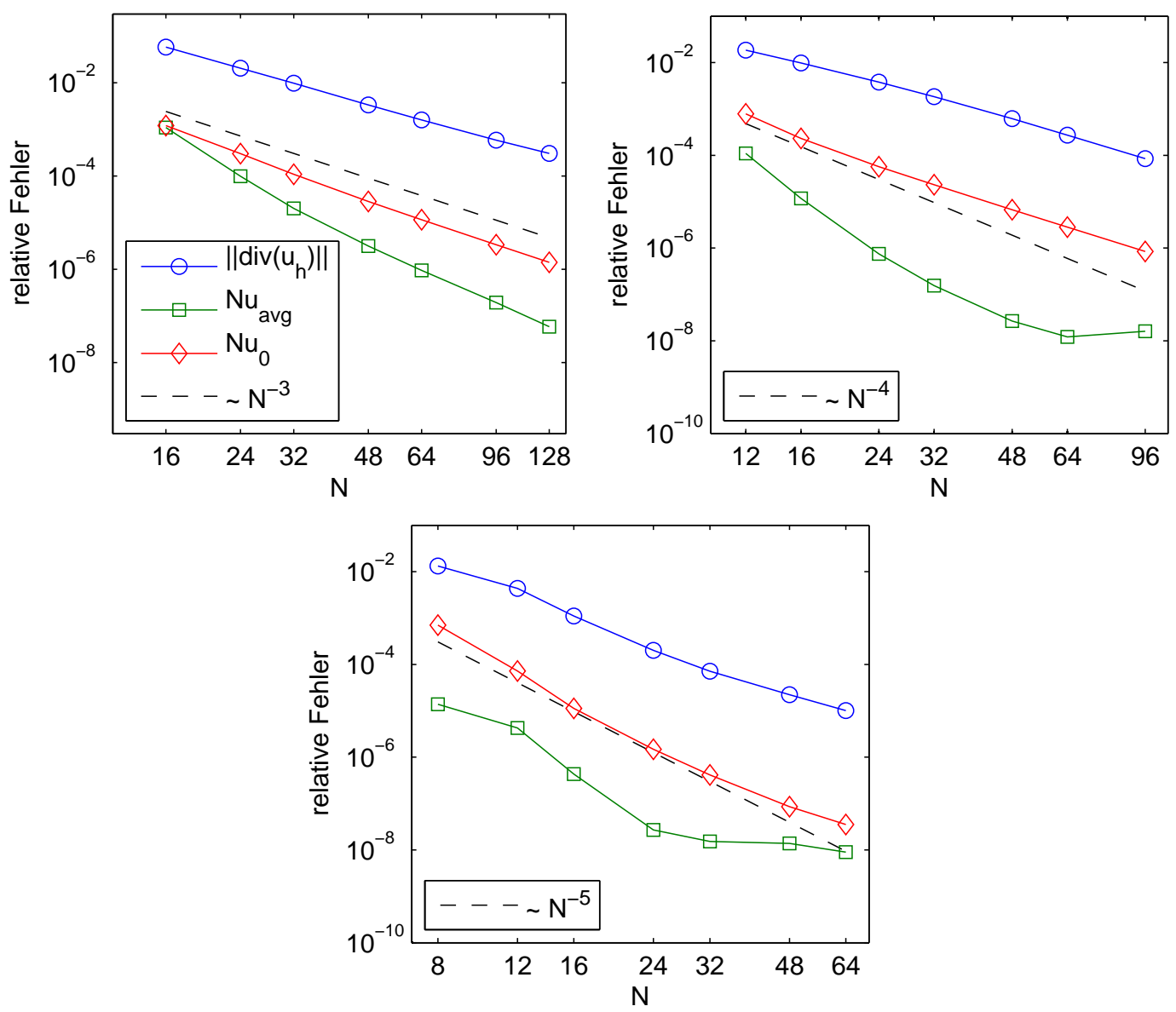

Abbildung 7.7: Konvergenz ausgewählter Größen für Ra $=10^{6}$ auf anisotropen Gittern mit $N^{2}$ Zellen und $\mathcal{Q}_{2} / \mathcal{Q}_{1} / \mathcal{Q}_{2}$-Elementen (oben links), $\mathcal{Q}_{3} / \mathcal{Q}_{2} / \mathcal{Q}_{3}$-Elementen (oben rechts) und $\mathcal{Q}_{4} / \mathcal{Q}_{3} / \mathcal{Q}_{4}$-Elementen (unten).

Erhöhung der Elementordnung. Ergebnisse für Elemente der Ordnung $k=3$ und $k=4$ sowie Ra $\in\left\{10^{6}, 10^{7}\right\}$ sind in Tabelle 7.5 auf Seite 119 angegeben. Abbildung 7.7 zeigt einen Vergleich der Konvergenzraten bei $\mathrm{Ra}=10^{6}$ für alle verwendeten Elementordnungen. Da es sich um anisotrope Gitter mit variierendem Seitenverhältnis der Zellen handelt, lässt sich diesen Gittern keine aussagekräftige Gitterweite $h$ zuordnen. Zum besseren Vergleich sind daher jeweils die Konvergenzraten $N^{-3}, N^{-4}$ bzw. $N^{-5}$ mit eingetragen. Für $\mathrm{Nu}_{\text {avg }}$ erreichen die Elemente höherer Ordnung bei $N=64$ bzw. $N=32$ bereits die Rechengenauigkeit. Die anderen Werte konvergieren weiter, allerdings teilweise mit reduzierter Konvergenzordnung.

In Abbildung 7.8 ist die Konvergenz für $\mathrm{Ra}=10^{7}$ dargestellt. Selbst bei dieser großen Rayleigh-Zahl erreicht die Diskretisierung mit $\mathcal{Q}_{4} / \mathcal{Q}_{3} / \mathcal{Q}_{4}$-Elementen bei $N=48$ für $\mathrm{Nu}_{\text {avg }}$ die numerische Rechengenauigkeit. 

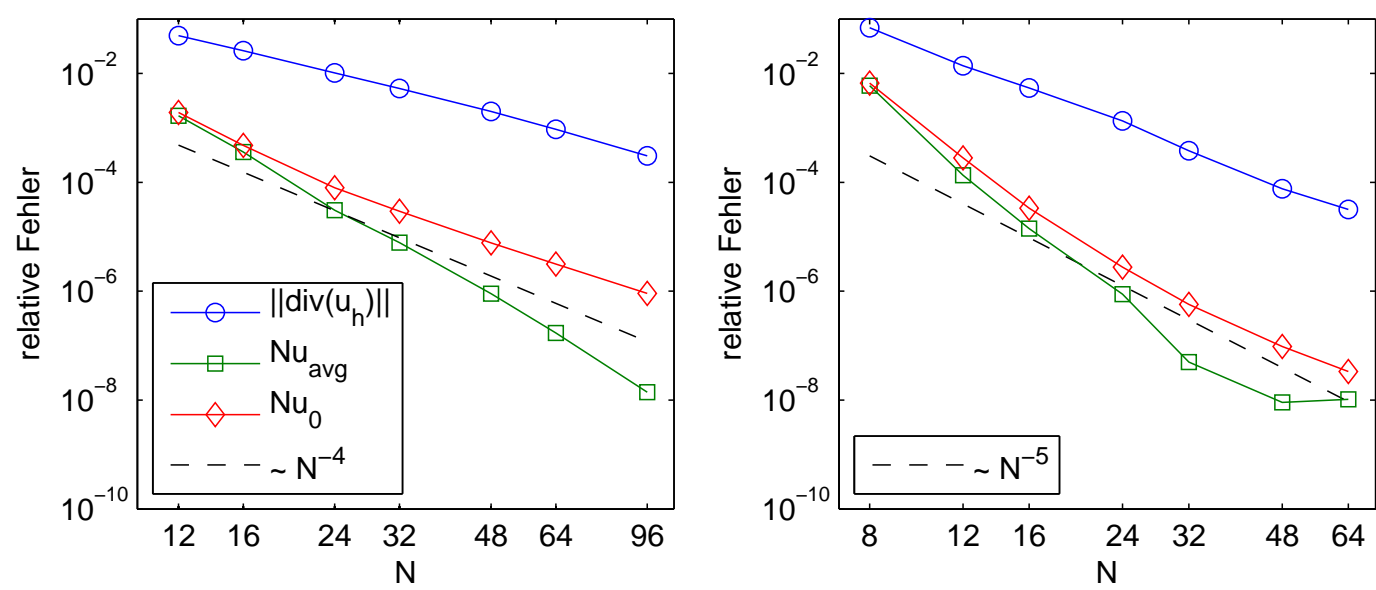

Abbildung 7.8: Konvergenz ausgewählter Größen für Ra $=10^{7}$ auf anisotropen Gittern mit $N^{2}$ Zellen und $\mathcal{Q}_{3} / \mathcal{Q}_{2} / \mathcal{Q}_{3}$-Elementen (links) und $\mathcal{Q}_{4} / \mathcal{Q}_{3} / \mathcal{Q}_{4}$-Elementen (rechts).

\begin{tabular}{ccccccc}
\hline $\mathrm{Ra}$ & $\sqrt{\mathrm{Ra}} u_{\max }$ & $\sqrt{\mathrm{Ra}} v_{\max }$ & $\mathrm{Nu}_{\min }$ & $\mathrm{Nu}_{\max }$ & $\mathrm{Nu}$ & $\sqrt{\mathrm{Ra}} \Phi_{\max }$ \\
\hline $10^{4}$ & 16.1833 & 19.6282 & 0.58496 & 3.53105 & 2.2448158 & 5.073673 \\
& $\approx 5 \times 10^{-6}$ & $\approx 5 \times 10^{-6}$ & $\approx 5 \times 10^{-5}$ & $\approx 1 \times 10^{-6}$ & $\approx 1 \times 10^{-8}$ & $\approx 2 \times 10^{-7}$ \\
\hline $10^{5}$ & 34.7407 & 68.6358 & 0.72795 & 7.72012 & 4.5216360 & 9.6164 \\
& $\approx 3 \times 10^{-6}$ & $\approx 3 \times 10^{-6}$ & $\approx 3 \times 10^{-5}$ & $\approx 1 \times 10^{-6}$ & $\approx 1 \times 10^{-8}$ & $\approx 1 \times 10^{-5}$ \\
\hline $10^{6}$ & 64.8344 & 220.5651 & 0.97944 & 17.5360 & 8.8252016 & 16.810 \\
& $\approx 8 \times 10^{-7}$ & $\approx 5 \times 10^{-7}$ & $\approx 1 \times 10^{-5}$ & $\approx 1 \times 10^{-6}$ & $\approx 1 \times 10^{-8}$ & $\approx 3 \times 10^{-5}$ \\
\hline $10^{7}$ & 148.585 & 699.330 & 1.3663 & 39.395 & 16.523093 & 30.164 \\
& $\approx 7 \times 10^{-6}$ & $\approx 2 \times 10^{-6}$ & $\approx 8 \times 10^{-5}$ & $\approx 3 \times 10^{-5}$ & $\approx 1 \times 10^{-8}$ & $\approx 5 \times 10^{-4}$ \\
\hline
\end{tabular}

Tabelle 7.1: Benchmarkwerte und geschätzte relative Fehler.

In Tabelle 7.1 sind extrapolierte Benchmarkwerte für Ra $\in\left\{10^{4}, 10^{5}, 10^{6}, 10^{7}\right\}$ angegeben. Für kleine Rayleigh-Zahlen sind diese deutlich genauer als die Werte aus den älteren Arbeiten [17, 65]. Für Ra $=10^{6}, 10^{7}$ ist die Übereinstimmung mit den Werten aus $[26,44]$ sehr gut. Die Genauigkeit der Benchmarkwerte konnte insbesondere für die Nusselt-Zahl jedoch deutlich gesteigert werden.

\subsection{Druckseparation}

In diesem Abschnitt soll die Auswirkung der Druckseparation (siehe Abschnitt 2.5) untersucht werden. In Abbildung 7.9 a) bis d) wird dazu der Druck und der zellweise Divergenzfehler für Rechnungen mit $\mathcal{Q}_{2} / \mathcal{Q}_{1} / \mathcal{Q}_{2}$-Elementen auf Gittern mit $N^{2}=256$ 


\begin{tabular}{ccccc}
\hline$N$ & Standard & Variante A & Variante B & Grad-Div \\
\hline 16 & 0.058520 & 0.051035 & 0.050350 & 0.007430 \\
32 & 0.009804 & 0.009119 & 0.009082 & 0.002589 \\
\hline
\end{tabular}

Tabelle 7.2: Divergenzfehler $\left\|\nabla \cdot \mathbf{u}_{h}\right\|_{0}$ für zwei Varianten der Druckseparation und für Divergenzstabilisierung mit $\gamma_{K}=0,3$.

Zellen und $\mathrm{Ra}=10^{6}$ dargestellt. In Teil a) der Abbildung werden die Werte für eine Rechnung ohne Grad-Div-Stabilisierung und ohne Druckseparation gezeigt. Es ist deutlich zu erkennen, dass der Druck durch einen globalen quadratischen Term in der $y$-Koordinate dominiert wird. Dieser entsteht durch den aus dem annähernd linearen vertikalen Temperaturverlauf resultierenden Auftriebsterm der Boussinesq-Approximation. Da der Ansatzraum für den Druck nur stückweise lineare Funktionen enthält, verursacht dieser quadratische Anteil einen Approximationsfehler, der sich insbesondere auf $\nabla \cdot \mathbf{u}_{h}$ auswirkt.

Zur Berechnung eines approximierten Drucks wird

$$
\min _{A \in \mathbb{R}} \int_{\Omega}(\theta-A(y-0.5))^{2} \mathrm{~d} \mathbf{x}
$$

bestimmt. Mit dem Minimalargument $A$ kann dann ein reduzierter Druck berechnet werden, der den quadratischen Anteil nicht mehr enthält (Variante A). Das Ergebnis dieser Rechnung ist in Teil b) von Abbildung 7.9 zu sehen. Der reduzierte Druck kann deutlich besser approximiert werden, wodurch auch der Divergenzfehler kleiner wird. In einem weiteren Schritt kann zusätzlich der kubische Anteil des Temperaturverlaufes durch

$$
\min _{B, C \in \mathbb{R}} \int_{\Omega}\left(\theta-B(y-0.5)-C(y-0.5)^{3}\right)^{2} \mathrm{~d} \mathbf{x}
$$

bestimmt werden. Die Subtraktion dieses Anteils (Variante B) verbessert die Divergenzfehler jedoch kaum noch, wie die Tabelle 7.2 zeigt. Die Hauptursache für den verbleibenden Divergenzfehler sind die Trägheitskräfte $\left(\mathbf{u}_{h} \cdot \nabla\right) \mathbf{u}_{h}$, die hauptsächlich im Bereich der Ecken wirken, in denen sich die Strömung von den vertikalen Wänden ablöst und die Richtung ändert.

Um den Divergenzfehler in allen Bereichen zu verbessern, kann die Grad-DivStabilisierung verwendet werden. Teil d) von Abbildung 7.9 zeigt die Ergebnisse für eine Rechnung mit $\gamma_{K}=0,3$ und ohne Druckseparation. Die zellweisen Divergenzfehler sind gegenüber der unstabilisierten Rechnung deutlich kleiner und konzentrieren sich weniger stark in den Ecken des Gebiets.

Für das vorliegende Problem ist der Einfluss der Druckseparation eher gering und deutlich schwächer als der Effekt von Grad-Div-Stabilisierung. Für eine Diskretisierung mit inf-sup stabilen Elementen und bei Beibehaltung des gekoppelten Problems für Geschwindigkeit und Druck ist eine Druckseparation daher nicht erforderlich. 

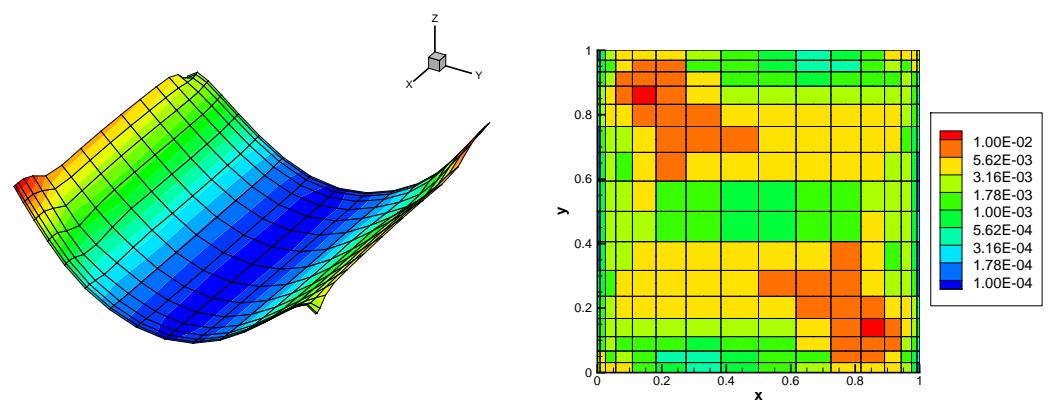

(a) Rechnung ohne Modifikation,
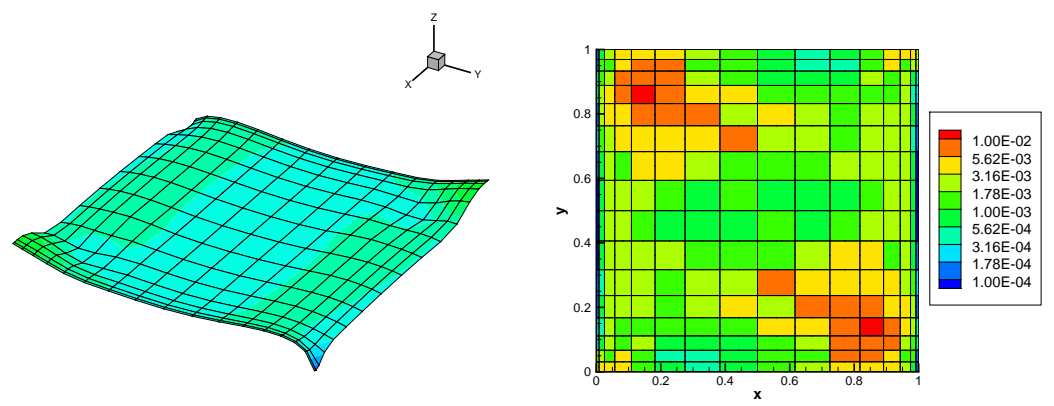

(b) mit Subtraktion des linearen Anteils des Boussinesq-Terms,
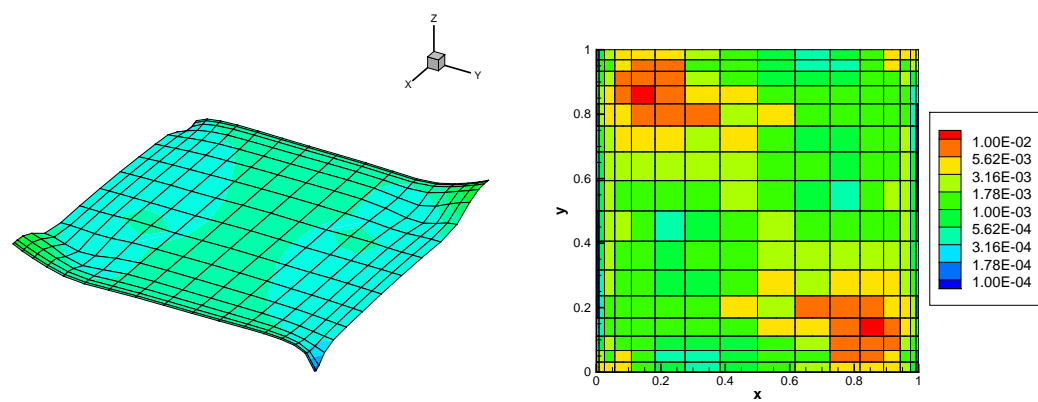

(c) mit Subtraktion des linearen und kubischen Anteils des Boussinesq-Terms,
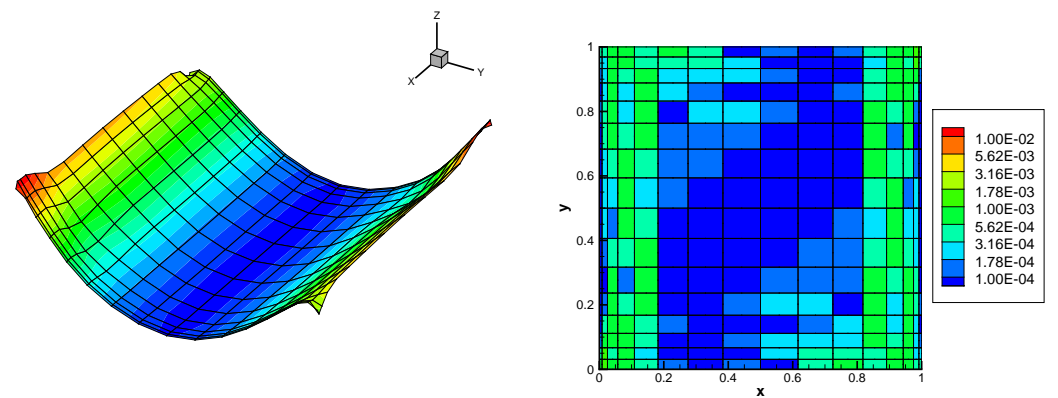

(d) und mit Grad-Div-Stabilisierung mit $\gamma_{K}=0.3$.

Abbildung 7.9: Druck (links) und zellweiser Divergenzfehler (rechts). 
Bemerkung 7.2 Wird das gekoppelte Problem für Geschwindigkeit und Druck durch ein Projektionsverfahren entkoppelt, kann die Druckseparationsmethode wesentliche Vorteile bringen. Im ersten Schritt dieser Verfahren wird z.B. mit einem alten Druck des letzten Zeitschritts die Impulsgleichung ohne Einhaltung der Divergenzbedingung gelöst. Danach wird das Geschwindigkeitsfeld zurück in den Raum der diskret divergenzfreien Funktionen $\mathbf{Z}_{h}$ projiziert. Je besser die Approximation des Drucks im ersten Schritt ist, desto genauer arbeiten diese Verfahren. 


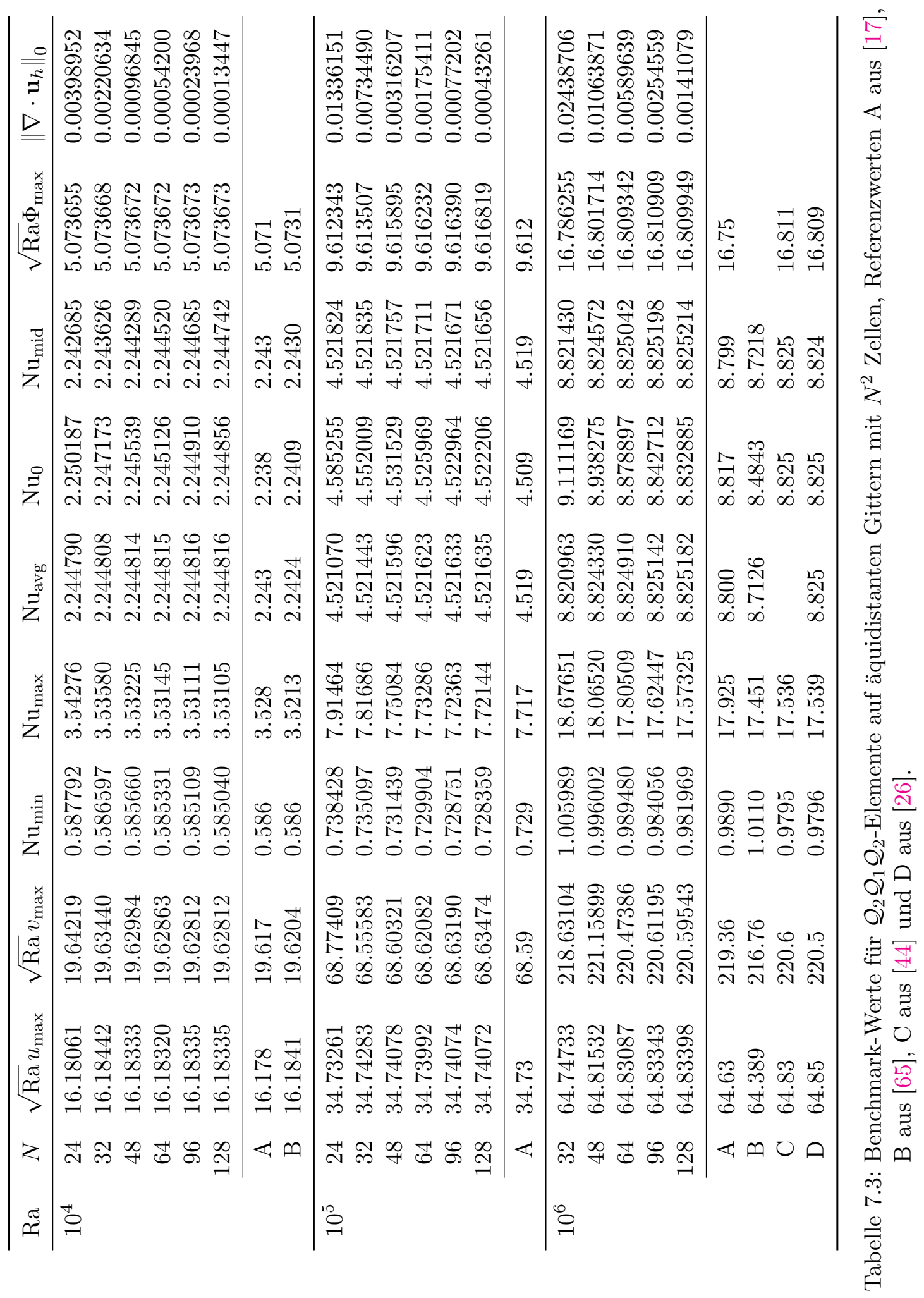




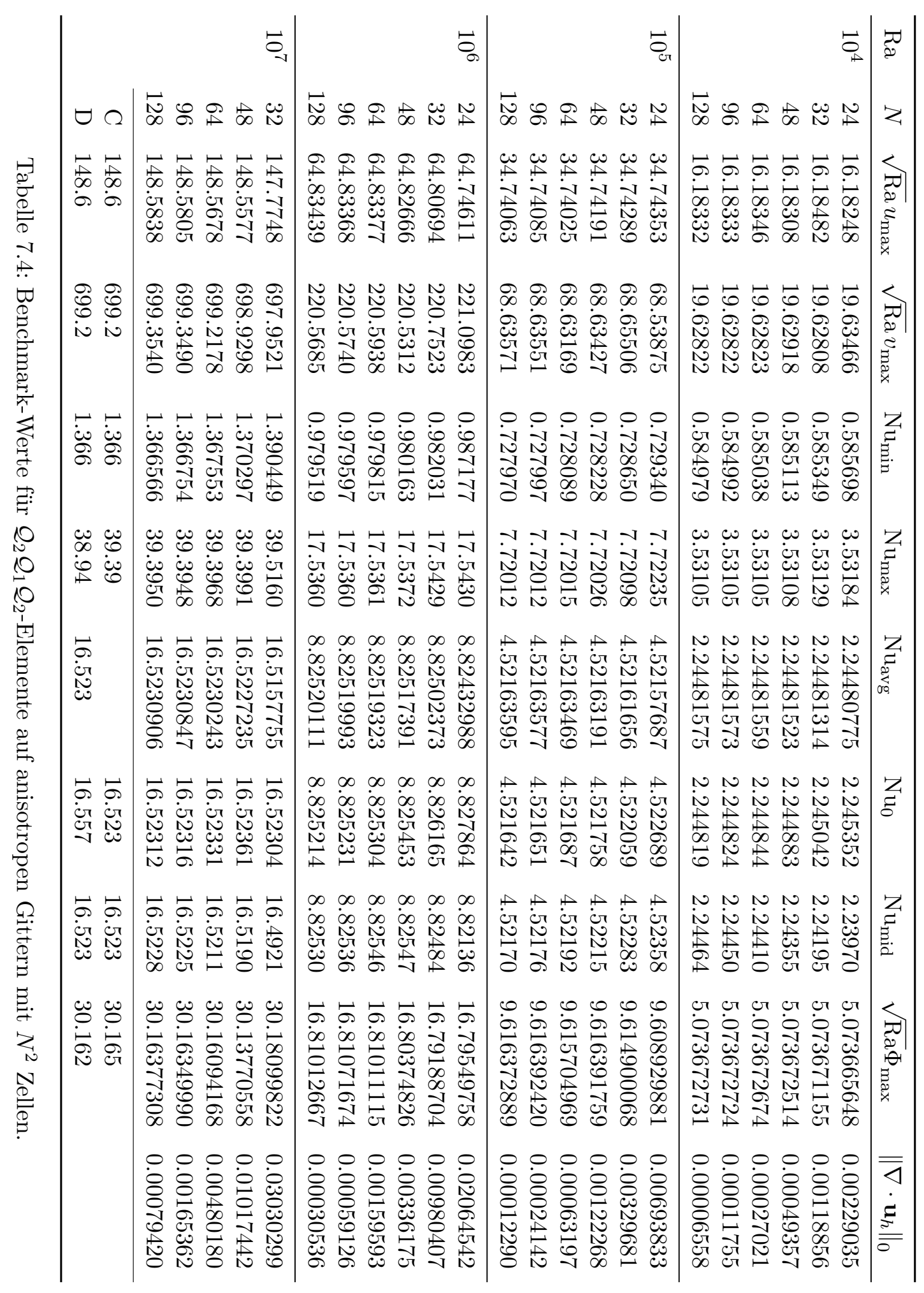




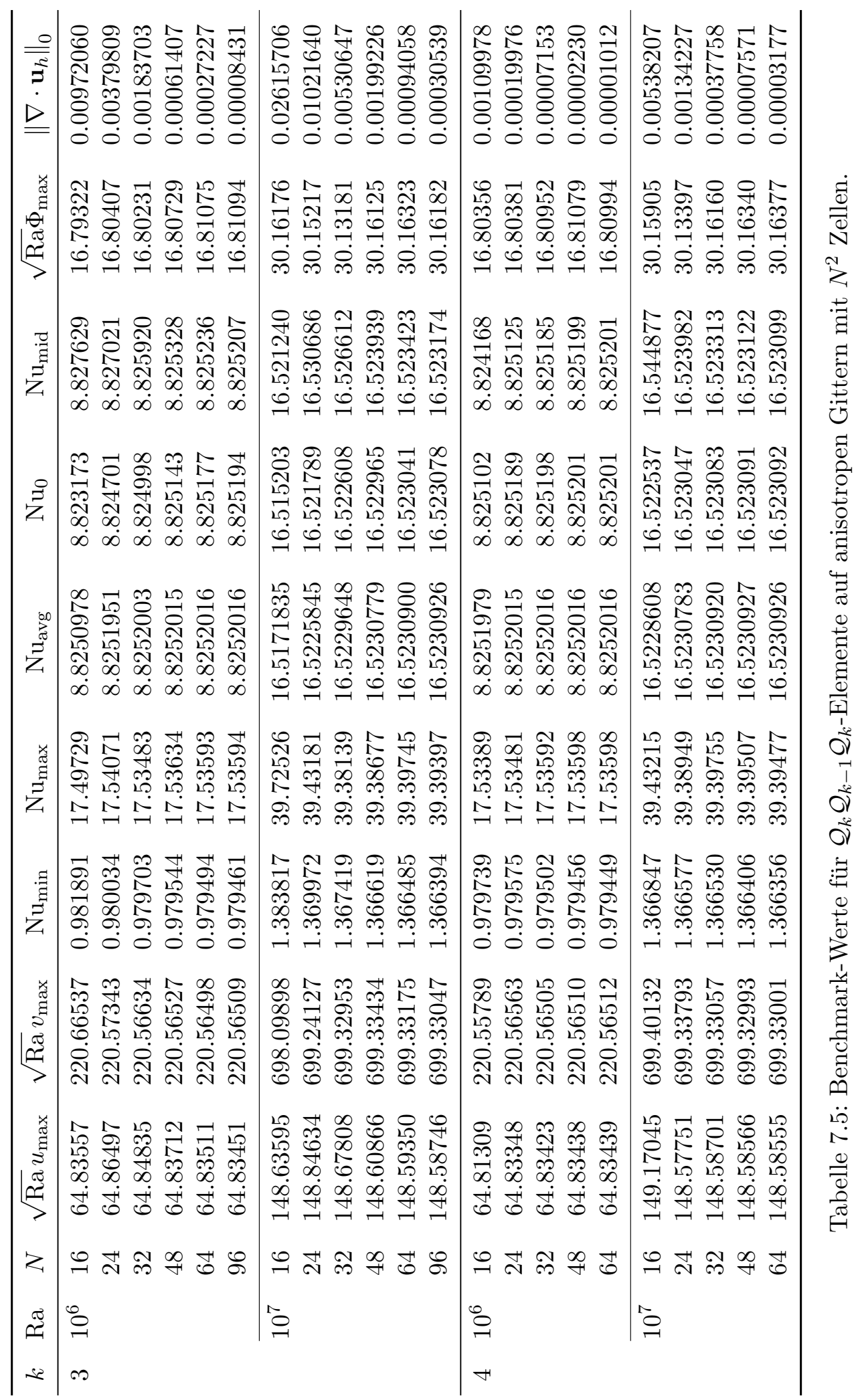




\section{Kapitel 8}

\section{Turbulente nicht-isotherme Hohlraumströmung}

Nach den Untersuchungen zu laminaren Hohlraumströmungen im vorherigen Kapitel wird nun der Fall einer turbulenten Hohlraumströmung bei Ra $=1,58 \times 10^{9}$ betrachtet. Dabei handelt es sich um ein verbreitetes Testproblem, das sowohl experimentell in den Arbeiten [2] und [69], als auch numerisch in den Arbeiten [58] und [40] gut untersucht wurde. Für die folgenden Rechnungen werden das Lilly-Eidson-Turbulenzmodell und erstmals für nicht-isotherme Strömungen das VMS-Turbulenzmodell verwendet.

\subsection{Beschreibung des Experiments}

Die folgende Beschreibung des Versuchsaufbaus ist Ref. [2] entnommen. Es handelt sich um eine luftgefüllte Kammer mit quadratischem Querschnitt, Breite und Höhe sind 0,75 m. Die Länge der Kammer ist 1,5 m, so dass die Strömung in der Mitte der Kammer bis auf kleine Störungen zweidimensional ist. Die vertikalen Seitenwände werden bei konstant $10 \pm 0,15{ }^{\circ} \mathrm{C}$ bzw. $50 \pm 0,15{ }^{\circ} \mathrm{C}$ gehalten, während die Raumtemperatur $30 \pm 0,2{ }^{\circ} \mathrm{C}$ beträgt. Die resultierende Rayleigh-Zahl wird mit $1,58 \times 10^{9}$ angegeben.

Die horizontalen Seitenwände oben und unten bestehen aus einem 1,5 mm dicken Stahlblech und $10 \mathrm{~cm}$ Styropor. Ihre thermischen Eigenschaften sind in der Beschreibung des Experiments jedoch nicht genau angegeben und lassen sich bei der numerischen Rechnung somit nicht befriedigend modellieren. Stattdessen werden, wie auch bei den anderen numerischen Studien [40,58], interpolierte Daten des Experiments als DirichletRandwerte verwendet.

Bei der Auswertung der experimentellen Daten ist zu beobachten, dass die Symmetrie zwischen heißer und kalter Seitenwand deutlich gestört ist. Eine mögliche Ursache hierfür könnte der große Temperaturunterschied von $40{ }^{\circ} \mathrm{C}$ sein, bei dem sich wesent- 

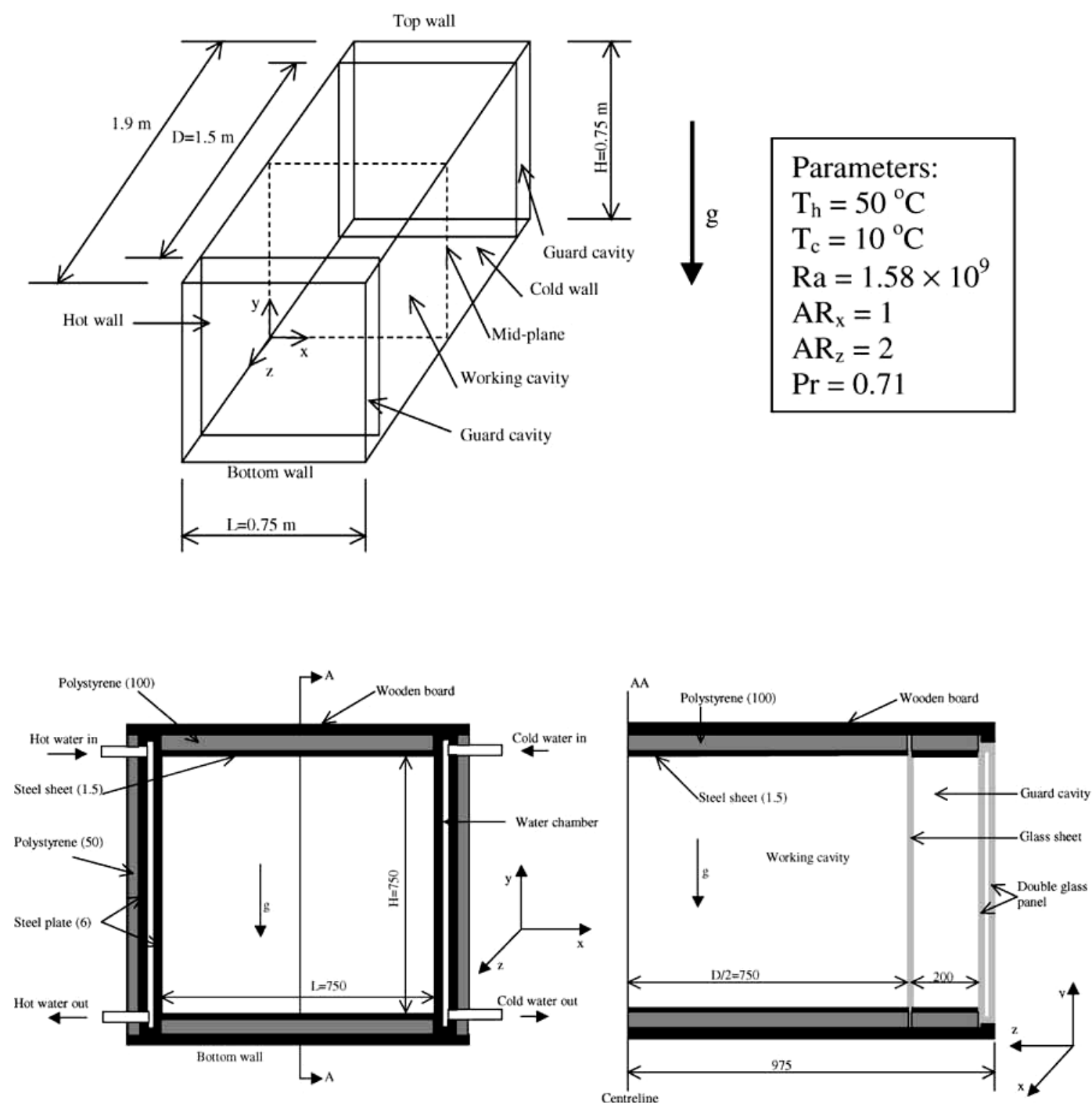

Abbildung 8.1: Abbildung zum Versuchsaufbau der thermisch angetriebenen Hohlraumströmung aus Ref. [2]. 


\begin{tabular}{cccccc}
\hline Temperatur & Dichte & $\begin{array}{c}\text { Kinematische } \\
\text { Viskosität }\end{array}$ & $\begin{array}{c}\text { Expansions- } \\
\text { koeffizient } \\
\beta\end{array}$ & $\begin{array}{c}\text { Prandtl- } \\
\text { Zahl } \\
T\end{array}$ & $\begin{array}{c}\text { Rayleigh- } \\
\text { Zahl }\end{array}$ \\
$\begin{array}{ccccc}\text { Pr } \\
\mathrm{kg} / \mathrm{m}^{3}\end{array}$ & $\mathrm{~m}^{2} / \mathrm{s} \times 10^{-6}$ & $1 / \mathrm{K} \times 10^{-3}$ & - & $10^{9}$ \\
\hline 10 & 1.247 & 14.21 & 3.532 & 0.7176 & 2.0779 \\
20 & 1.205 & 15.11 & 3.411 & 0.7156 & 1.7698 \\
30 & 1.165 & 16.04 & 3.299 & 0.7138 & 1.5152 \\
40 & 1.128 & 16.98 & 3.193 & 0.7121 & 1.3055 \\
50 & 1.093 & 17.95 & 3.095 & 0.7105 & 1.1298 \\
\hline
\end{tabular}

Tabelle 8.1: Physikalische Eigenschaften von (Raum-) Luft nach http://www.mhtl. uwaterloo.ca/old/onlinetools/airprop/airprop.html.

liche physische Eigenschaften von Luft zum Teil um mehr als $25 \%$ ändern (z.B. kinematische Viskosität, siehe Tabelle 8.1). Das verwendete Oberbeck-Boussinesq-Modell berücksichtigt diese Änderungen nicht und verwendet konstante mittlere Werte. Diese werden in Übereinstimung mit den Angaben zum Experiment so gewählt, dass bei einer Prantl-Zahl von $\operatorname{Pr}=0,71$ eine Rayleigh-Zahl von $\mathrm{Ra}=1,58 \times 10^{8}$ erreicht wird.

Die experimentellen Daten werden zum besseren Vergleich symmetrisiert. Im Experiment ist ein kleinerer Temperaturunterschied bei gleicher Rayleigh-Zahl nur durch eine technisch aufwendigere, größere Kammer zu erreichen. Entsprechende Versuche sind jedoch nicht verfügbar. Der vorliegende Temperaturunterschied und die daraus resultierende Dichtevariation liegen gerade noch in dem Bereich, für den das verwendete OberbeckBoussinesq-Modell als anwendbar betrachtet wird.

\subsection{Wahl der Filterweite}

Für die Turbulenzmodelle müssen die turbulenten Viskositäten $\nu_{K}^{T}$ und $\alpha_{K}^{T}$ festgelegt werden. Es muss neben $C_{E}$ (siehe Abschnitt 3.4.4) auch die Filterweite $\delta$ bestimmt werden. Dazu gibt es verschiedene Möglichkeiten. In Abbildung 8.2 ist zur Veranschaulichung eine dreidimensionale anisotrope Gitterzelle mit den Bezeichnungen für die Gitterparameter skizziert. Eine skalare Filterweite $\delta_{K}>0$ für jede Zelle $K$ lässt sich auf unterschiedliche Weisen wählen:

$$
\begin{array}{lll}
\text { Durchmesser: } & \delta_{K} \sim \operatorname{diam}(K)=\left(h_{K, 1}^{2}+h_{K, 2}^{2}+h_{K, 3}^{2}\right)^{1 / 2}=h_{K}, \\
\text { kürzeste Kante: } & \delta_{K} \sim h_{K, \min }=\min _{i=1, \ldots, d} h_{K, i}, \\
\text { längste Kante: } & \delta_{K} \sim h_{K, \max }=\max _{i=1, \ldots, d} h_{K, i}, \\
\text { geometrisches Mittel: } & \delta_{K} \sim \operatorname{vol}(K)^{1 / 3}=\left(h_{1} h_{2} h_{3}\right)^{1 / 3} .
\end{array}
$$



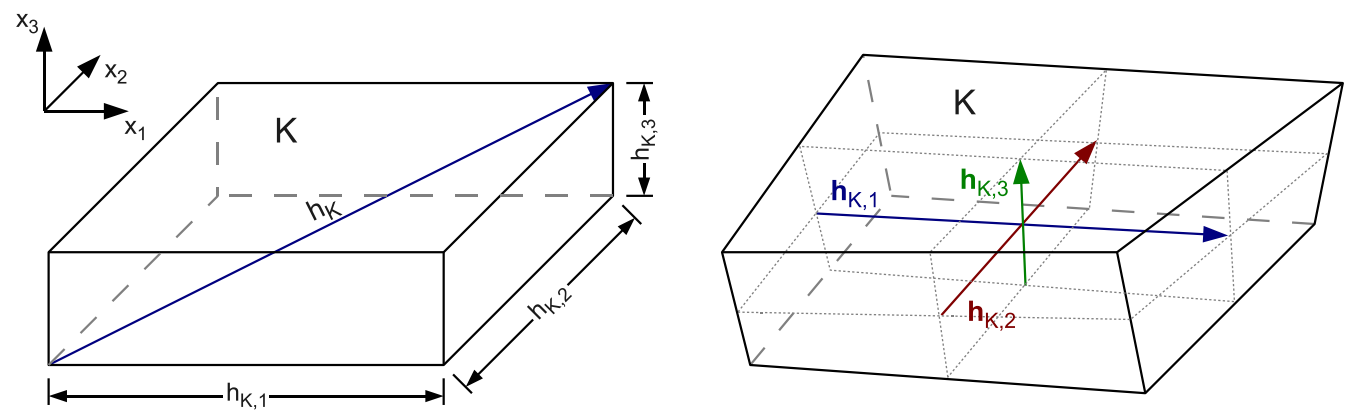

Abbildung 8.2: Anisotrope Gitterzellen $K$ und Gitterparameter.

Am Beispiel eines Gitters, das nur in eine Koordinatenrichtung verfeinert wird, lassen sich jedoch Nachteile dieser Definitionen aufzeigen. Der Durchmesser $h_{K}$ und die längste Kante $h_{K, \max }$ ändern sich bei wiederholter Verfeinerung in nur eine Richtung wenig bzw. gar nicht. Die vorhandene höhere Aufösung des Gitters in diese Richtung wird vom Modell nicht erkannt und kleine Strukturen entlang dieser Richtung werden durch die zu große Diffusion unnötig gedämpft. Die Verwendung von $h_{K \text {,min }}$ führt in der Regel nicht zu einem stabilen Turbulenzmodell, da die turbulente Viskosität nicht ausreicht, um Fluktuationen in die anderen Koordinatenrichtungen zu dämpfen. Die Wahl des geometrischen Mittels führt für die beschriebene Verfeinerung auf die willkührliche Skalierung $\delta_{K} \sim h_{K \text {,min }}^{1 / 3}$, bzw. für zweidimensionale Gitter auf $\delta_{K} \sim h_{K \text {,min }}^{1 / 2}$, und ist somit dimensionsabhängig.

Soll die Lösung des Modells unabhängig von einer anisotropen Transformation des Gebiets und von der Einführung einer zusätzlichen homogenen Koordinate sein, scheidet somit die Verwendung einer skalaren Filterweite aus. Wir betrachten daher eine Modifikation des Turbulenzmodells mit einem anisotropen Multiskalen-Diffusionsoperator. Dazu ersetzen wir die bisherigen Modellterme (3.31) durch anisotrope Varianten. Exemplarisch für die Temperaturgleichung lautet dieser Term:

$$
\sum_{K \in \mathcal{T}_{h}} \widehat{\alpha}_{K}^{T}\left(\delta_{K}\left[\kappa_{\theta}\left(\nabla \theta_{h}\right)\right], \delta_{K}\left[\kappa_{\theta}\left(\nabla \psi_{h}\right)\right]\right)_{K} .
$$

Dabei ist für jedes $K \in \mathcal{T}_{h}$ die Filterweite

$$
\delta_{K}=\left(\begin{array}{ccc}
h_{K, 1} & 0 & 0 \\
0 & h_{K, 2} & 0 \\
0 & 0 & h_{K, 3}
\end{array}\right) \in \mathbb{R}^{d \times d}
$$

jetzt eine anisotrope Skalierungsmatrix und $\widehat{\alpha}_{K}^{T}$ ist wie in (3.25), jedoch ohne das Quadrat der Filterweite als Faktor, definiert. Handelt es sich nicht um eine kartesische Zelle, so kann $\delta_{K}$ durch die Vektoren $\mathbf{h}_{K, i}, i=1, \ldots, d$, die die Zentren gegenüberliegender Seitenflächen der Zelle $K$ verbinden (siehe Abbildung 8.2 rechts), definiert werden:

$$
\delta_{K}=\left(\mathbf{h}_{K, 1}, \mathbf{h}_{K, 2}, \mathbf{h}_{K, 3}\right) \in \mathbb{R}^{d \times d} .
$$


Die Reihenfolge der Vektoren $\mathbf{h}_{K, i}$ und ihre Orientierung spielt keine Rolle, da

$$
\left(\delta_{K}\left[\kappa_{\theta}\left(\nabla \theta_{h}\right)\right], \delta_{K}\left[\kappa_{\theta}\left(\nabla \psi_{h}\right)\right]\right)_{K}=\sum_{i=1, \ldots, d}\left(\mathbf{h}_{K, i} \cdot \kappa_{\theta}\left(\nabla \theta_{h}\right), \mathbf{h}_{K, i} \cdot \kappa_{\theta}\left(\nabla \psi_{h}\right)\right)_{K}
$$

unabhängig von beidem ist. Der anisotrope Diffusionsterm setzt sich also aus Diffusionstermen für die einzelnen Richtungen $\mathbf{h}_{K, i}$ zusammen, deren Viskositätsparameter an die Auflösung des Gitters in die jeweilige Richtung angepasst ist.

\subsection{Zeitliche Mittelung}

Auch für die turbulente Hohlraumströmung wird die Simulation mit einem ruhenden Fluid und einem linearen Temperaturverlauf von der warmen zur kalten Wand gestartet. Die Startphase wird lang genug gewählt, so dass sich eine statistisch stationäre Strömung einstellt. Die Turbulenz entwickelt sich automatisch durch Diskretisierungsfehler und Residuen der algebraischen Lösungsverfahren. Ein spezielle Anregung von Turbulenz (z.B. duch gestörte Startwerte) war nicht nötig. Die Startphase der Rechnung umfasst etwa 2500 Zeitschritte mit einer Zeitschrittweite von $\tau=0,125$, was etwa 20 vollständigen Strömungsumläufen entspricht. Das Intervall, über das die Lösung danach gemittelt wird, umfasst mindestens 1000 weitere Zeitschritte.

\subsection{Zweidimensionale Rechnungen}

Bei Turbulenz handelt es sich um ein dreidimensionales Phänomen. Die grundlegenden Mechanismen, die zum Energietransfer von den großen zu den kleinen Skalen führen und schließlich das Kolmogorovsche 5/3-Gesetz begründen, funktionieren in zweidimensionalen Strömungen nicht. Experimentelle Untersuchungen in Ref. [2] zeigen jedoch, dass es sich bei der betrachteten Hohlraumströmung um eine statistisch zweidimensionale Strömung handelt. Die Fluktuationen in der dritten Raumkoordinate entstehen erst im mittleren Skalenbereich innerhalb der Energiekaskade, während die energiereichen Strukturen zweidimensional sind. Da die Large Eddy Simulation nur die Simulation dieser Strukturen zum Ziel hat und der Einfluss der kleineren Skalen durch das Turbulenzmodell modelliert wird, werden wir zunächst zweidimensionale Rechnungen durchführen.

Wie für die laminaren Rechnungen werden auch im turbulenten Fall anisotrope Gitter verwendet. Wegen der benötigten großen Anzahl an Zeitschritten wird die Auflösung jedoch auf $N=64$ und die Elementordnung auf $k=2$ begrenzt.

Für das Lilly-Eidson-Modell sind die Ergebnisse in Abbildung 8.3 dargestellt und werden mit experimentellen Werten aus [2] verglichen. Im oberen Teil der Abbildung sind ein vertikaler und ein horizontaler Temperaturquerschnitt zu sehen. Auch für die turbulente Strömung ist deutlich ein linearer vertikaler Temperaturverlauf zu sehen. Die beiden mittleren Abbildungen zeigen logarithmische Darstellungen der Geschwindigkeit und 

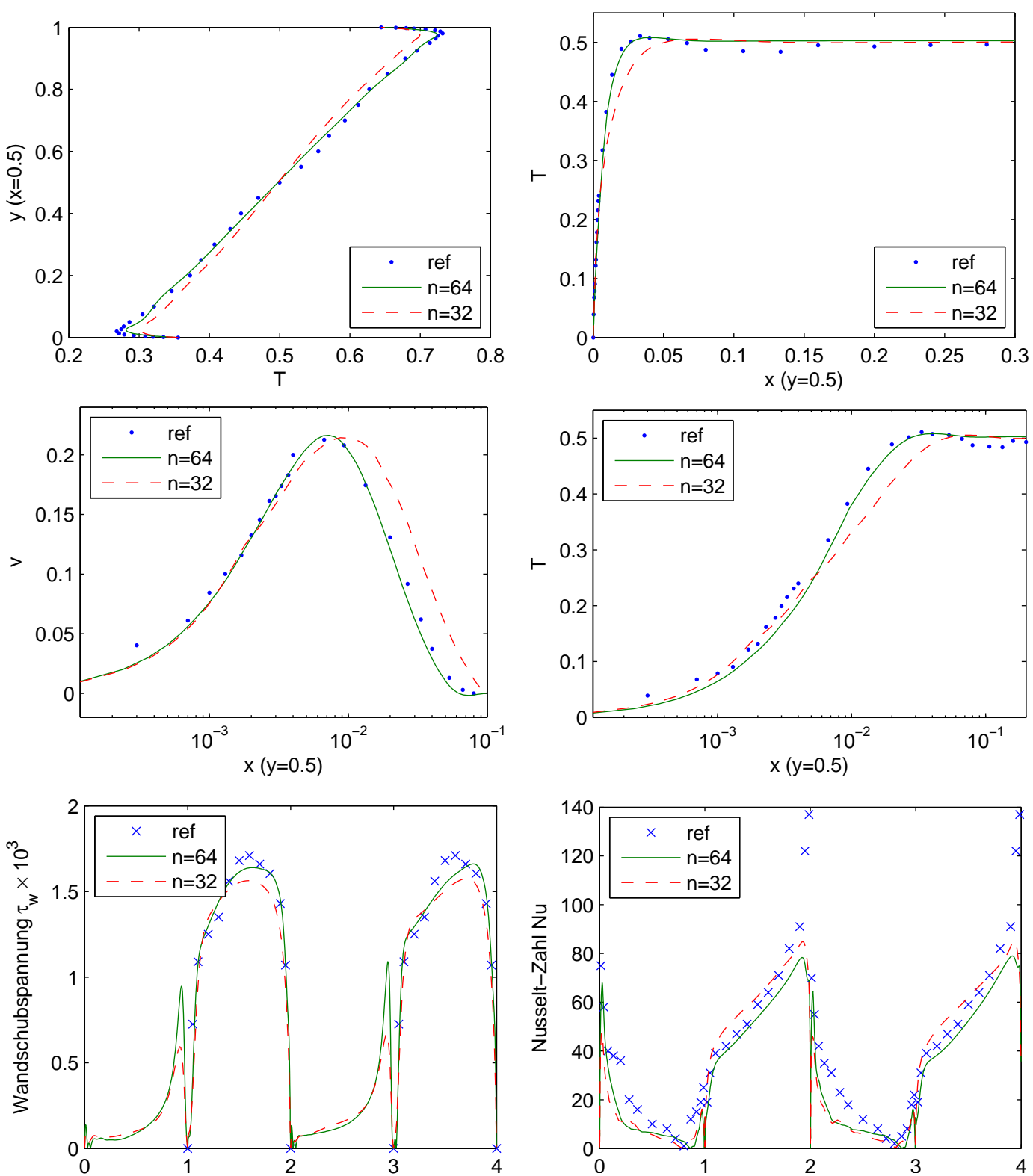

Abbildung 8.3: Lösungen mit Lilly-Eidson-Modell, Temperaturquerschnitte (oben), logarithmische Darstellung der Grenzschichten an der warmen Wand (mitte) sowie Wandschubspannung und Nusselt-Zahl an der warmen Wand (unten). 

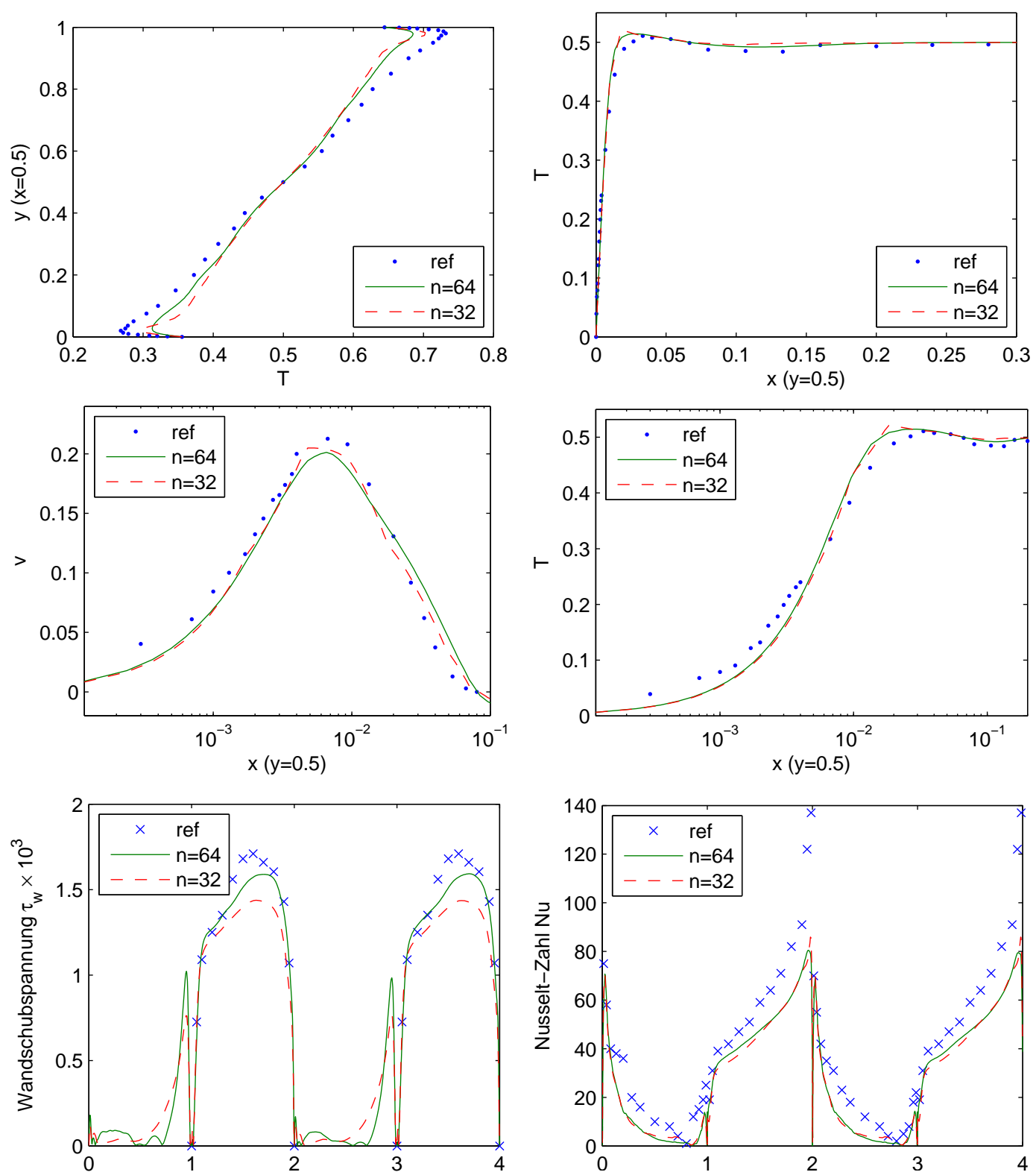

Abbildung 8.4: Lösungen mit VMS-Turbulenzmodell, Temperaturquerschnitte (oben), logarithmische Darstellung der Grenzschichten an der warmen Wand (mitte) sowie Wandschubspannung und Nusselt-Zahl an der warmen Wand (unten). 


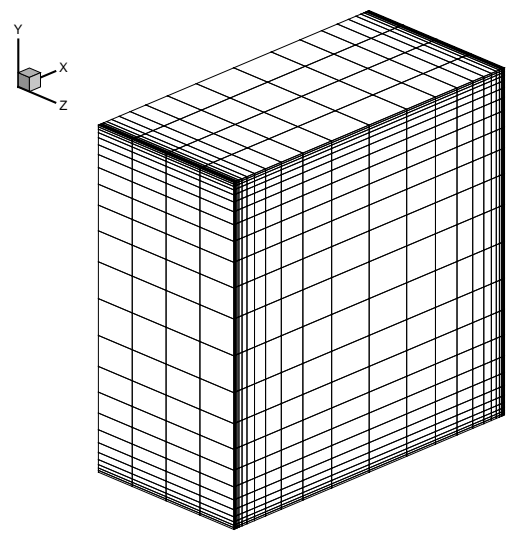

Abbildung 8.5: Beispielgitter mit 24x24x4 Zellen für die dreidimensionalen Rechnungen.

der Temperatur in den vertikalen Grenzschichten an der warmen Wand. Insbesondere dort ist eine starke Gitterabhängigkeit der Lösung zu erkennen. Diese wird nicht durch die zu geringe Gitteraufösung verursacht, sondern durch die gitterabhängige Dissipation des Turbulenzmodells. Auf dem feinen Gitter wird eine gute Übereinstimmung mit den Referenzwerten erreicht. Im unteren Teil der Abbildung sind die Wandschubspannung und die Nusselt-Zahl entlang des Umfangs der Kammer (im mathematisch positiven Drehsinn) aufgetragen. Auch hier zeigt sich die Gitterabhängigkeit der Lösung.

In Abbildung 8.4 sind die gleichen Ergebnisse für das VMS-Turbulenzmodell dargestellt. In den Grenzschichtprofilen zeigt sich das Modell deutlich weniger gitterabhängig. Die Abweichungen von den Referenzwerten für die Wandschubspannung und die Nusselt-Zahl sind etwas größer als beim Lilly-Eidson-Modell und zeigen ebenfalls eine stärkere Gitterabhängigkeit. Das kann dadurch begründet werden, dass für das VMS-Turbulenzmodell kein van-Driest-damping verwendet und das vollständige Turbulenzmodell bis zur Wand hin angewendet wird. Die Abweichungen im vertikalen Temperaturverlauf lassen sich dadurch jedoch nicht erklären.

\subsection{Dreidimensionale Rechnungen}

Für die dreidimensionalen Rechnungen wird das Gebiet in $z$-Richtung als periodisch betrachtet. Zur Begrenzung des Rechenaufwands müssen außerdem gröbere Gitter verwendet werden. In Abbildung 8.5 ist exemplarisch ein Gitter mit $24 \times 24 \times 4$ Zellen dargestellt. Für eine feinere Rechnung wird ein Gitter mit $32 \times 32 \times 8$ Zellen verwendet. Das entspricht bei der Verwendung von $\mathcal{Q}_{2} / \mathcal{Q}_{1} / \mathcal{Q}_{2}$-Elementen insgesamt $4 \cdot\left(49^{2} \cdot 9\right)+25^{2} \cdot 5=89561$ bzw. $4 \cdot\left(65^{2} \cdot 17\right)+33^{2} \cdot 9=297101$ Unbekannten.

Wir betrachten gemittelte Lösungen auf dem zentralen Querschnitt $z=0$ für das VMSTurbulenzmodell in Abbildung 8.6. Die Übereinstimmung der Lösungen auf den groben 

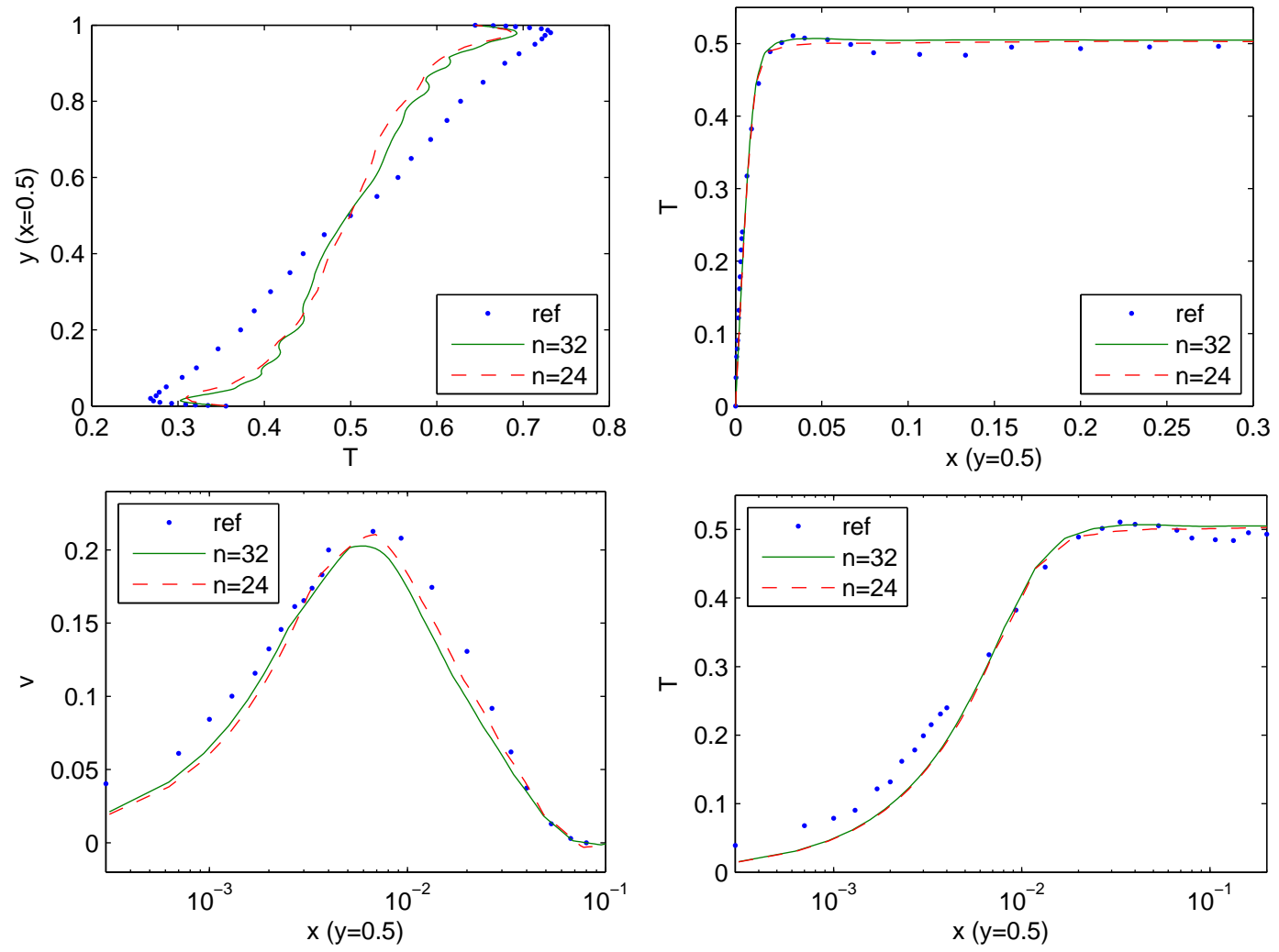

Abbildung 8.6: VMS Turbulenzmodell, Temperaturquerschnitte (oben) und logarithmische Darstellung der Grenzschichten an der warmen Wand (unten) für $z=0$.

dreidimensionalen Gittern ist für die Grenzschichten an den vertikalen Wänden erneut gut. Auch zeigt sich wieder der geringe Einfluss der Gitterauflösung auf die Lösung. Wie schon bei den zweidimensionalen Rechnungen sind jedoch größere Abweichungen beim vertikalen Temperaturverlauf zu erkennen. Insgesamt sind die Unterschiede zwischen den zwei- und dreidimensionalen Rechnungen gering. Dies zeigt, dass statistisch zweidimensionale Strömungen bei Verwendung eines Turbulenzmodells auch zweidimensional gerechnet werden können.

\subsection{Untersuchung des Energiespektrums}

Bisher wurden lediglich zeitlich gemittelte Größen betrachtet. In diesem Abschnitt sollen auch Energiespektren untersucht werden. Da das Problem jedoch keine homogene Koordiantenrichtung besitzt, kann das Energiespektrum nicht durch eine FourierTransformation berechnet werden. Stattdessen verwenden wir Taylor's frozen turbulence hypothesis (siehe Ref. [59]). Es wird angenommen, dass die turbulenten Fluk- 

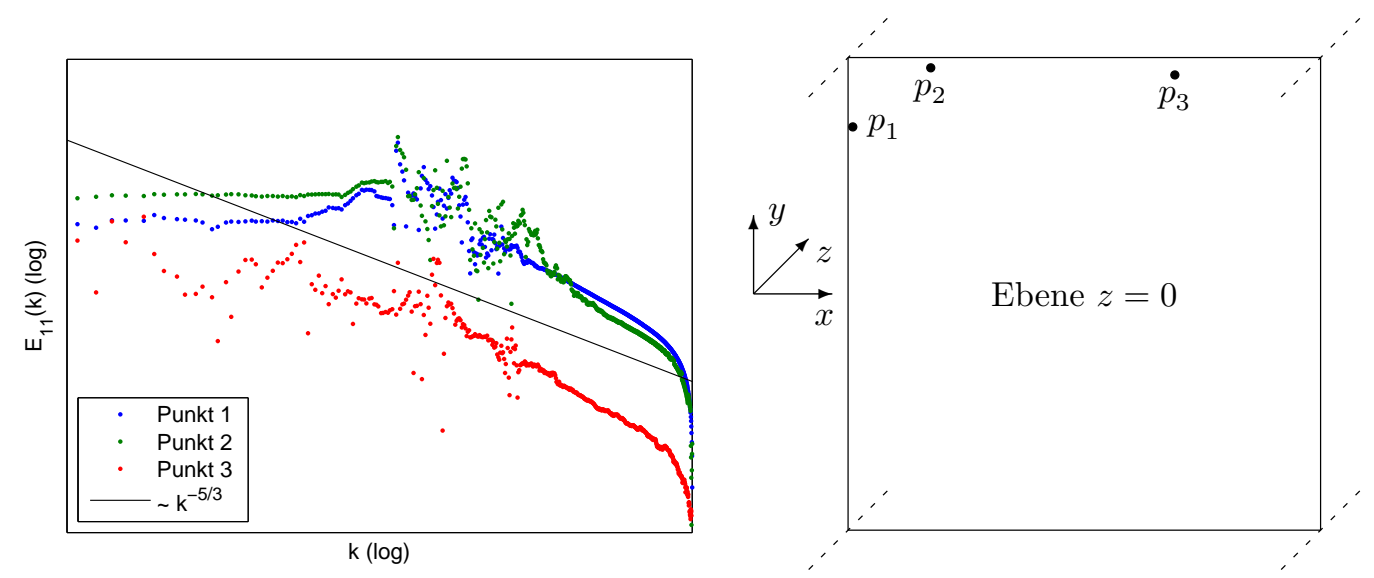

Abbildung 8.7: Longitudinales Energiespektrum $E_{11}(k)$ in drei Messpunkten und Skizze der Lage der Messpunkte.

tuationen auf die gemittelte Strömung aufgesetzt sind und sich, während sie einen Punkt passieren, nicht verändern. Dann können wir den Geschwindigkeitsvektor in einem stationären Punkt messen, an dem die turbulenten Fluktuationen mit der mittleren Strömungsgeschwindigkeit vorbeitransportiert werden. So kann ein eindimensionales longitudinales Energiespektrum $E_{11}(k)$ in Richtung der mittleren Strömungsrichtung berechnet werden. Für das vollständige Energiespektrum $E(k)$ lässt sich für isotrope Turbulenz folgende Abhängigkeit von $E_{11}(k)$ zeigen:

$$
E(k)=\frac{1}{2} k^{3} \frac{\mathrm{d}}{\mathrm{d} k}\left(\frac{1}{k} \frac{\mathrm{d} E_{11}(k)}{\mathrm{d} k}\right) .
$$

Daraus folgt, dass auch $E_{11}(k)$ im mittleren Skalenbereich das gleiche Potenzgesetz

$$
E_{11}(k)=K_{1} \varepsilon^{2 / 3} k^{-5 / 3}, \quad K_{1}=\frac{18}{55} K_{0}
$$

wie $E(k)$ erfüllt. Wir werden $E_{11}(k)$ aus Messungen in drei Punkten berechnen. Die Lage der Messpunkte ist zusammen mit den Ergebnissen in Abbildung 8.7 dargestellt.

Die Energiespektren zeigen deutlich, dass es sich nicht um eine periodische Strömung oder eine Überlagerung mehrerer periodischer Strömungen handelt. Solche Strömungen besitzen diskrete Energiespektren bei denen sich die Energie auf wenige isolierte Wellenzahlen verteilt. Die numerische Simulation entspricht also tatsächlich einer turbulenten Strömung.

Für kleinere Wellenzahlen zeigen die Energiespektren teilweise starke Schwankungen. Diese sind möglicherweise auf Rezirkulationszonen zurückzuführen, die Fluktuationen mit niedriger Frequenz in der Strömung verursachen. Für größere Wellenzahlen wird annähernd das Kolmogorovsche 5/3-Gesetz reproduziert. 


\section{Abschlussbetrachtung}

Die vorliegende Arbeit enthält ein komplettes Programm zur Implementierung eines Strömungslösers für nicht-isotherme inkompressible Strömungsprobleme. Es wird die Wahl des mathematischen Modells, der räumlichen Diskretisierung, des Turbulenzmodells und der zeitlichen Diskretisierung behandelt.

Als mathematisches Modell wird das Oberbeck-Boussinesq-Modell verwendet. Dazu werden in Kapitel 1 neuere Resultate aus [20] zitiert, die zeigen, dass es sich bei diesem Modell um einen speziellen singulären Grenzwert des deutlich allgemeineren NavierStokes-Fourier-Modells handelt. Das Oberbeck-Boussinesq-Modell steht somit auf einem stabilen mathematischen Fundament.

Um turbulente Strömungen zu berechnen, ohne alle Skalen aufzulösen, muss das Strömungsmodell um ein Turbulenzmodell erweitert werden. Das Forschungsgebiet der Turbulenzmodellierung befindet sich dabei im stetigen Wandel. Durch die zunehmende Rechenleistung von Computersystemen wird der Wunsch geweckt, nicht nur statistisch gemittelte Lösungen zu berechnen, sondern einen Teil der turbulenten Strukturen auch zeitabhängig zu simulieren. Dazu sind robuste und gleichzeitig genaue Turbulenzmodelle nötig, die sich zudem effizient implementieren lassen. In dieser Arbeit wird daher erstmals ein auf einem Multiskalen-Diffusionsoperator basierendes LES-Modell auf nichtisotherme Strömungen angewendet, das sich zuvor in ersten numerischen Versuchen mit isothermen Strömungen bewährt hat.

Die Analysis für Lösungen im Raum diskret divergenzfreier Funktionen in Kapitel 4 umfasst die Turbulenzmodellterme und stellt eine Erweiterung bestehender Analysis für den isothermen Fall dar. Die Fehlerabschätzungen für das semi-diskrete Problem basieren auf hohen räumlichen Regularitätsannahmen, die auch die Existenz und Eindeutigkeit der Lösungen sicherstellen. Ein kurzer Beweis für die Eindeutigkeit der Lösung wird ebenfalls in Kapitel 4 geführt. Ansonsten handelt es sich bei der Frage nach der Existenz und Eindeutigkeit von Lösungen der inkompressiblen Navier-Stokes-Gleichungen bei niedriger Regularität weiterhin um eines der offenen Millenium-Probleme.

Die a-priori Fehlerabschätzung enthält einen exponentiell in der Zeit anwachsenden Term, der sich wegen der nötigen Abschätzungen für die nicht-linearen Terme nicht vermeiden lässt. Ein exponentielles Anwachsen der Fehler ist wegen der deutlich besseren Stabilitätsabschätzungen jedoch nur für kurze Zeit möglich. Aber nur in speziellen 
Fällen, in denen sich das Problem dissipativ verhält, sind bessere Abschätzungen ohne exponentielles Anwachsen des Fehlers möglich. Eine sorgfältigere Abschätzung mit höherem technischen Aufwand könnte jedoch zu einer Verbesserung der Abschätzung hinsichtlich der Abhängigkeit von den Daten führen.

In dieser Arbeit wird der Ansatz verfolgt, die Zeitdiskretisierung und die räumliche Diskretisierung unabhängig voneinander zu betrachten. Eine kombinierte voll diskrete Analysis ist meist technisch aufwendig und bringt kaum neue Erkenntnisse über das Zusammenspiel beider Diskretisierungen. Stattdessen können die Eigenschaften des semi-diskreten Systems untersucht werden und Konzepte wie Stabilität oder der Index algebraischer Variablen zur Auswahl geeigneter Verfahren verwendet werden. An dieser Schnittstelle fehlen allerdings einige Konzepte, um alle Eigenschaften der Verfahren bei Anwendung auf die nicht-lineare Impulsgleichung zu beschreiben. Insbesondere die Interaktion verschiedener Skalenbereiche wird bisher durch lineare Testprobleme und Stabilitätsbegriffe nicht erfasst.

Ausführliche numerische Untersuchungen werden in den letzten drei Kapiteln der Arbeit vorgestellt. Erst wird die zeitabhängige Umströmung eines Zylinders, ein isothermes Benchmarkproblem mit zeitabhängigen Randbedingungen und Referenzwerten, untersucht. Der Schwerpunkt liegt dabei auf der zeitlichen Diskretisierung. Im Ergebnis stellt sich das sBDF-Verfahren, bei dem es sich um eine Modifikation eines Verfahrens aus [5] handelt, als effizientestes Verfahren heraus. Es realisiert neben der Zeitdiskretisierung gleichzeitig die Linearisierung des Gleichungssystems.

Am Beispiel laminarer nicht-isothermer Hohlraumströmungen mit stationären Lösungen werden ausführliche Gitterkonvergenzstudien durchgeführt. Der Schwerpunkt liegt dabei auf der Auswertung der Nusselt-Zahl, einer dimensionslosen Kenngröße für nichtisotherme Strömungen. Die Genauigkeit von Benchmarkwerten aus älteren Arbeiten konnte so zum Teil erheblich gesteigert werden. Die Finite-Elemente-Methode mit inf-sup stabilen Elementen erweist sich dabei als sehr robust und gleichzeitig präzise. Sie erfordert keine Kalibrierung von problemabhängigen Tuningparametern und lässt sich dank ihrer Flexibilität bei der Vernetzung von Gebieten auch leicht für komplexe Geometrie und Strömungsprobleme anwenden.

Im letzten Kapitel wird eine nicht-isotherme Hohlraumströmung bei großer RayleighZahl $\mathrm{Ra}=1,58 \times 10^{9}$ untersucht, für die sich eine zeitabhängige turbulente Strömung einstellt. In numerischen Versuchen wird dabei das erstmals auf nicht-isotherme Strömungen angewendete VMS-Turbulenzmodell mit dem etablierten Lilly-Eidson-Turbulenzmodell und experimentellen Messwerten verglichen. Die Ergebnisse sind vielversprechend und eine weitere Untersuchung derartiger Turbulenzmodelle wird empfohlen. Insbesondere auch eine adaptive Grobraumwahl und eine dynamische Berechnung verschiedener turbulenter Parameter, wie sie schon für andere Turbulenzmodelle untersucht wurde, scheinen geeignet, um die Genauigkeit der Methode weiter zu verbessern. 


\section{Symbolverzeichnis}

\begin{tabular}{ll}
\hline Symbol & Bezeichnung \\
\hline$\alpha$ & Temperaturleitfähigkeit \\
$\beta$ & thermischer Expansionskoeffizient \\
$\delta$ & Filterweite \\
$\epsilon$ & kleiner Störungsparameter \\
$\kappa_{u}, \kappa_{\theta}$ & Fluktuationsoperatoren \\
$\mu$ & Viskosität \\
$\nu$ & kinematische Viskosität \\
$\varrho$ & Massendichte \\
$\sigma$ & Entropieproduktionsrate \\
$\tau$ & Zeitschrittweite \\
$\theta$ & Temperatur \\
$\vartheta$ & absolute Temperatur \\
$\Omega \subset \mathbb{R}^{d}$ & Gebiet \\
$\Theta \subseteq H^{1}(\Omega)$ & Funktionenraum für Temperatur \\
\hline $\mathbf{g}=(0,0, g)^{\top}$ & Gravitationsvektor \\
$\mathbf{n}$ & äußerer Einheitsnormalenvektor \\
$\mathbf{q}$ & Wärmestrom \\
$\mathbf{u}=(u, v, w)^{\top}, \mathbf{v}, \mathbf{w}$ & Geschwindigkeitsfeld \\
$\mathbf{x}=(x, y, z)^{\top}$ & Raumkoordianten \\
\hline$b_{s}, c_{s}$ & Trilinearformen der Konvektionsterme \\
$c_{p}$ & spezifische Wärmekapazität bei konstantem Druck \\
$d$ & Raumdimension \\
$e$ & spezifische innere Energie \\
$h$ & Gitterweite \\
$h_{K}$ & Zelldurchmesser \\
$k$ & Wärmeleitfähigkeit oder Wellenzahl \\
$p, q$ & Druck und Testfunktion \\
$s$ & spezifische Entropie \\
$t$ & Zeit \\
\hline & \\
\hline
\end{tabular}




\begin{tabular}{ll}
\hline Symbol & Bezeichnung \\
\hline $\mathrm{m}$ & Meter \\
$\mathrm{s}$ & Sekunde \\
$\mathrm{K}$ & Kelvin \\
$\mathrm{kg}$ & Kilogramm \\
$\mathrm{N}$ & Newton \\
\hline $\mathcal{Q}, \mathcal{P}$ & Tensorprodukt- und Standardpolynomraum \\
$\mathcal{T}_{h}$ & Triangulierung \\
$B, B^{\top}$ & Gradient- bzw. Divergenzoperator \\
$F_{K}: \hat{K} \rightarrow K$ & Zelltransformation \\
$\hat{K}, K \subset \mathbb{R}^{d}$ & Referenzzelle bzw. Gitterzelle \\
$M_{u}, M_{\theta}$ & Massematrizen \\
$Q=L_{*}^{2}(\Omega)$ & Funktionenraum für Druck \\
$\mathbf{V} \subseteq\left[H^{1}(\Omega)\right]^{d}$ & Funktionenraum für Geschwindigkeit \\
$\mathbf{Z} \subsetneq \mathbf{V}$ & Raum divergenzfreier Geschwindigkeitsfelder \\
\hline $\mathbb{C}$ & Komplexe Zahlen \\
$\mathbb{N}$ & Natürliche Zahlen \\
$\mathbb{R}$ & Reelle Zahlen \\
$\mathbb{S}$ & Spannungstensor \\
\hline $\mathrm{Fr}$ & Froude-Zahl \\
$\mathrm{Gr}$ & Grashof-Zahl \\
$\mathrm{Ma}$ & Mach-Zahl \\
$\mathrm{Nu}$ & Nusselt-Zahl \\
$\mathrm{Pe}$ & Péclét-Zahl \\
$\mathrm{Pr}$ & Prandtl-Zahl \\
$\mathrm{Ra}$ & Rayleigh-Zahl \\
$\operatorname{Re}$ & Strouhal-Zahl \\
$\mathrm{Sr}$ &
\end{tabular}




\section{Literaturverzeichnis}

[1] R.A. Adams. Sobolev Spaces. Academic Press, 1975.

[2] F. Ampofo and T. G. Karayiannis. Experimental benchmark data for turbulent natural convection in an air filled square cavity. International Journal of Heat and Mass Transfer, 46:3551-3572, 2003.

[3] U. M. Ascher and L. R. Petzold. Computer methods for ordinary differential equations and differential-algebraic equations. Society for Industrial and Applied Mathematics, 1998.

[4] U.M. Ascher, S.J. Ruuth, and R.J. Spiteri. Implicit-Explicit Runge-Kutta Methods for Time-Dependent Partial Differential Equations. Applied Numerical Mathematics, 25:151-167, 1997.

[5] U.M. Ascher, S.J. Ruuth, and B. Wetton. Implicit-Explicit Methods for TimeDependent Partial Differential Equations. SIAM Journal on Numerical Analysis, 32(3):797-823, 1995.

[6] W. Bangerth, R. Hartmann, and G. Kanschat. deal.II - a general purpose object oriented finite element library. ACM Trans. Math. Softw., 33(4):24/1-24/27, 2007.

[7] W. Bangerth and G. Kanschat. deal. II Differential Equations Analysis Library, Technical Reference. http://www.dealii.org.

[8] L.C. Berselli, T. Iliescu, and W.J. Layton. Mathematics of Large Eddy Simulation of Turbulent Flows. Springer, 2006.

[9] M. Braack, G. Lube, and L. Röhe. Divergence preserving interpolation on anisotropic quadrilateral meshes. Calcolo, submitted, 2011.

[10] James H. Bramble. A proof of the inf-sup condition for the stokes equations on lipschitz domains. Mathematical Models and Methods in Applied Sciences, 13:361$371,2003$.

[11] F. Brezzi. On the existence, uniqueness and approximation of saddle-point problems arising from lagrangian multipliers. RAIRO, 8:129-151, 1974. 
[12] E. Brown and G. Ahlers. Effect of the Earth's Coriolis force on the large-scale circulation of turbulent Rayleigh-Bénard convection. Physics of Fluids, 18:125108, 2006.

[13] A. J. Chorin. Solution of the Navier-Stokes Equations. Mathematics of Computation, 22:745-762, 1968.

[14] A. J. Chorin. Vorticity and turbulence. Springer, 2. überarbeitete Auflage, 1998.

[15] R. Codina, J. Principe, and M. Ávila. Finite element approximation of turbulent thermally coupled incompressible flows with numerical sub-grid scale modelling. International Journal of Numerical Methods for Heat \& Fluid Flow, 20:492-516, 2010.

[16] T.A. Davis. Algorithm 832: UMFPACK V4.3 - an unsymmetric-pattern multifrontal method. ACM Transactions on Mathematical Software, 30:196-199, Juni 2004.

[17] G. De Vahl Davis. Natural Convection of Air in a Square Cavity a Bench Mark Numerical Solution. International Journal for Numerical Methods in Fluids, 3:249264, 1983.

[18] P. Durbin and B. Pettersson Reif. Statistical theory and modeling for turbulent flows. Wiley, 2001.

[19] T. M. Eidson. Numerical simulation of the turbulent Rayleigh-Bénard problem using subgrid modelling. Journal of Fluid Mechanics, 158:245-268, 1985.

[20] E. Feireisl and A. Novotný. Singular limits in thermodynamics of viscous fluids. Advances in mathematical fluid mechanics. Birkhäuser, 2009.

[21] G.P. Galdi and W.J. Layton. Approximation of the larger eddies in fluid motion II: A model for space-filtered flow. Math. Models Methods Appl. Sci., 10(3):343-350, 2000 .

[22] S. Ganesan and V. John. Pressure separation - a technique for improving the velocity error in finite element discretisations of the Navier-Stokes equations. Applied Mathematics and Computation, 165:275-290, 2005.

[23] U. Ghia, K. N. Ghia, and C. T. Shin. High-Re solutions for incompressible flow using the Navier-Stokes equations and a multigrid method. Journal of Computational Physics, 48:387-411, 1982.

[24] V. Girault and P.A. Raviart. Finite element methods for Navier-Stokes equations: theory and algorithms. Springer Series in Computational Mathematics, Vol. 5. Springer Verlag, 1986.

[25] V. Girault and L.R. Scott. A quasi-local interpolation operator preserving the discrete divergence. Calcolo, 40:1-19, 2003. 
[26] T. Gjesdal, C.E. Wasberg, and A.P. Reif. Spectral element benchmark simulations of natural convection in two-dimensional cavities. INTERNATIONAL JOURNAL FOR NUMERICAL METHODS IN FLUIDS, 50:1297-1319, 2006.

[27] K. Goto and K. Milfeld. GotoBLAS2 Basic Linear Algebra Subroutines. http://www.tacc.utexas. edu/tacc-projects/gotoblas2/.

[28] H. Grant, R. Stewart, and A. Moilliet. Turbulence spectra from a tidal channel. Journal of Fluid Mechanics, 12:241-268, 1962.

[29] D. D. Gray and A. Giorgini. The validity of the boussinesq approximation for liquids and gases. Int. Journ. Heat Mass Transfer, 19:545-551, 1976.

[30] E. Hairer, S. P. Nørsett, and G. Wanner. Solving ordinary differential equations I: Nonstiff Problems. Springer Series in Computational Mathematics, Vol. 8. SpringerVerlag, 2. überarbeitete Auflage, 1993.

[31] E. Hairer and G. Wanner. Solving ordinary differential equations II: Stiff and Differential-Algebraic Problems. Springer Series in Computational Mathematics, Vol. 14. Springer-Verlag, 1991.

[32] T. Heister. Parallel Solvers for Incompressible Flow Problems. PhD thesis, GeorgAugust-Universität Göttingen, 2011.

[33] T. J. R. Hughes. Multiscale phenomena: Green's functions, the Dirichlet-toNeumann formulation, subgrid scale models, bubbles and the origins of stabilized methods. Computer Methods in Applied Mechanics and Engineering, 127:387-401, 1995.

[34] F. Incropera, D. DeWitt, T. Bergman, and A. Lavine. Fundamentals of Heat and Mass Transfer. Wiley, 6. überarbeitete Auflage, 2006.

[35] V. John. Parallele Lösung der inkompressiblen Navier-Stokes Gleichungen auf adaptiv verfeinerten Gittern. PhD thesis, Fakultät für Mathematik, Otto-von-GuerickeUniversität Magdeburg, 1997.

[36] V. John. Large Eddy Simulation of Turbulent Incompressible Flows: Analytical and Numerical Results for a Class of LES Models. Springer, 2004.

[37] V. John. Reference values for drag and lift of a two-dimensional time-dependent flow around a cylinder. International Journal for Numerical Methods in Fluids, 44:777-788, 2004.

[38] V. John and S. Kaya. Finite element error analysis of a variational multiscale method for the Navier-Stokes equations. Advances in Computational Mathematics, $28: 43-61,2008$.

[39] C.A. Kennedy and M.H. Carpenter. Additive Runge-Kutta schemes for convectiondiffusion-reaction equations. Applied Numerical Mathematics, 44:139-181, 2003. 
[40] T. Knopp. Finite-Element simulation of bouyancy-driven turbulent flows. $\mathrm{PhD}$ thesis, Georg-August-Universität Göttingen, 2003.

[41] A. Kolmogorov. The Local Structure of Turbulence in Incompressible Viscous Fluid for Very Large Reynolds Numbers. Royal Society (London), Proceedings, Series A - Mathematical and Physical Sciences, 434:9-13, 1991.

[42] W. Layton, C. Manica, M. Neda, M. Olshanskii, and L. Rebholz. On the accuracy of the rotation form in simulations of the Navier-Stokes equations. J. Comput. Phys., 228:3433-3447, 2009.

[43] W. Layton and L. Tobiska. A Two-Level Method with Backtracking for the NavierStokes Equations. SIAM J. Numer. Anal, 35:2035-2054, 1998.

[44] P. Le Quéré. Accurate solutions to the square thermally driven square cavity at high Rayleigh number. Computers and Fluids, 20:29-41, 1991.

[45] P. Le Quéré and M. Behnia. From onset of unsteadiness to chaos in a differentially heated square cavity. Journal of Fluid Mechanics, 359:81-107, 1998.

[46] D.K. Lilly. On the numerical simulation of buoyant convection. Tellus, 14:148-172, 1962.

[47] D.K. Lilly. The representation of small-scale turbulence in numerical simulation experiments. Proceedings of IBM Scientific Computing Symp. on Environmental Sciences, 320:195-210, 1967.

[48] A. Linke. Divergence-Free Mixed Finite Elements for the Incompressible NavierStokes Equation. PhD thesis, Friedrich-Alexander-Universität Erlangen-Nürnberg, 2007.

[49] G. Lube. Numerische Mathematik I. Vorlesungsskript, Georg-August-Universität Göttingen, 2010.

[50] G. Lube and M. A. Olshanskii. Stable finite-element calculation of incompressible flows using the rotation form of convection. IMA Journal of Numerical Analysis, 22:437-461, 2002.

[51] G. Lube, G. Rapin, and J. Löwe. Local projection stabilization for incompressible flows: Equal-order vs. inf-sup stable interpolation. Electronic Transactions on Numerical Analysis, 32:106-122, 2008.

[52] J. Löwe. A locally adapting parameter design for the divergence stabilization of FEM discretizations of the Navier-Stokes equations, volume 69 of Lecture Notes in Computational Science and Engineering. Springer, 2009.

[53] J. Löwe and G. Lube. A projection-based variational multiscale method for largeeddy simulation with application to non-isothermal free-convection problems. Math. Model. Meths. Appl. Sc., akzeptiert im Mai 2011. 
[54] J. Löwe, G. Lube, and L. Röhe. A Projection-Based Variational Multiscale Method for the Incompressible Navier-Stokes/Fourier Model. Lecture Notes in Computational Science and Engineering, BAIL 2010 Proceedings, 81:167-175, 2011.

[55] G. Matthies, P. Skrzypacz, and L. Tobiska. A unified convergence analysis for local projection stabilisations applied to the Oseen problem. Mathematical Modelling and Numerical Analysis, 41:713-742, 2007.

[56] A. Oberbeck. Über die Wärmeleitung der Flüssigkeiten bei Berücksichtigung der Strömung infolge von Temperaturdifferenzen. Annalen der Physik, 243(6):271-292, 1879.

[57] M. Olshanskii, G. Lube, T. Heister, and J. Löwe. Grad-div stabilization and subgrid pressure models for the incompressible Navier-Stokes equations. Comput. Meth. Appl. Mech. Engrg., 198:3975-3988, 2009.

[58] S.H. Peng and L. Davidson. Comparison of subgrid-scale models in les for turbulent convection flow with heat transfer. 2nd EF Conference in Turbulent Heat Transfer, 1:5.24-5.35, 1998 .

[59] S. B. Pope. Turbulent flows. Cambridge Univ. Press, 2001.

[60] A. Quarteroni and A. Valli. Numerical approximation of partial differential equations. Springer series in computational mathematics, 1994.

[61] J. Rang. Design of DIRK schemes for solving the Navier-Stokes equations. Informatikbericht Nr. 2007-02, 2007.

[62] L. Röhe. Turbulence Modeling by a Projection-Based Variational Multiscale Method for Incompressible Flow Problems. PhD thesis, Georg-August-Universität Göttingen, 2011.

[63] L. Röhe and G. Lube. Analysis of a variational multiscale method for Large-Eddy simulation and its application to homogeneous isotropic turbulence. Computer $\mathrm{Me}$ thods in Applied Mechanics and Engineering, 199:2331-2342, 2010.

[64] P. Sagaut, S. Deck, and M. Terracol. Multiscale and Multiresolution Approaches in Turbulence. Imperial College Press, 2006.

[65] T. Saitoh and K. Hirose. High-accuracy bench mark solutions to natural convection in a square cavity. Computational Mechanics, 4:417-427, 1989.

[66] H. Schlichting and K. Gersten. Grenzschicht-Theorie. Springer, 10. überarbeitete Auflage, 2006.

[67] L.R. Scott and S. Zhang. Finite Element Interpolation of Nonsmooth Functions Satisfying Boundary Conditions. Math. Comp., 54:483-493, 1990.

[68] K. R. Sreenivasan. On the universality of the Kolmogorov constant . Physics of Fluids, 7:2778-2784, 1995. 
[69] Y.S. Tian and T.G. Karayiannis. Low turbulence natural convection in an air filled square cavity: Part I: the thermal and fluid flow fields. International Journal of Heat and Mass Transfer, 43:849-866, 2000.

[70] S. Turek. Efficient solvers for incompressible flow problems : an algorithmic and computational approach. Lecture Notes in Computational Science and Engineering. Springer, 1999.

[71] S. Turek and M. Schäfer. Benchmark computations of laminar flow around cylinder. 52:547-566, 1996. co. F. Durst, E. Krause, R. Rannacher.

[72] D. C. Wilcox. Turbulence Modeling for Cfd. D C W Industries, 3. überarbeitete Auflage, 2006.

[73] T. A. Zang. On the rotation and skew-symmetric forms for incompressible flow simulations. Applied Numerical Mathematics - Special issue: Transition to turbulence, 7:27-40, 1991. 


\section{Danksagung}

Mit der Fertigstellung dieser Arbeit endet für mich ein Lebensabschnitt, auf den ich gerne zurückblicke. Mein besonderer Dank gilt meinem Betreuer Herrn Professor Dr. Gert Lube für seine Unterstützung bei der Anfertigung und seine zahlreichen Anmerkungen zu dieser Arbeit.

Auch den weiteren Mitgliedern der Arbeitsgruppe "Numerische Methoden für singulär gestörte PDEs", insbesondere Lars Röhe, mit denen ich gemeinsam die Zeit der Promotion bestritten habe, möchte ich für die fruchtbaren Diskussionen, die gemeinsame Arbeit am Strömungslöser und das Korrekturlesen der Arbeit danken.

Dank gilt auch meiner Familie für die Unterstützung während meines gesamten Studiums und speziell meiner Frau, die die gesamte Arbeit korrekturgelesen hat.

Weiterhin möchte ich dem Graduiertenkolleg 1023 "Identifikation in mathematischen Modellen" für die Finanzierung von Konferenzreisen, unter anderem zur BAIL 2008 in Limerick (Irland) und zum FEM-Symposium 2009 in Oberwiesenthal, sowie für die Bewilligung eines Stipendiums für die Zeit vom September 2010 bis Juli 2011 danken. 


\section{Lebenslauf}

\begin{tabular}{|c|c|}
\hline \multicolumn{2}{|l|}{ Persönliche Daten: } \\
\hline & $\begin{array}{l}\text { Johannes Löwe } \\
\text { geboren am 28.6.1983 in Göttingen } \\
\text { deutscher Staatsbürger }\end{array}$ \\
\hline \multicolumn{2}{|l|}{ Bildungsweg: } \\
\hline September 2010 - Juli 2011 & Stipendiat des Graduiertenkollegs 1023 \\
\hline Juni 2008 - August 2010 & Assoziiertes Mitglied des GK 1023 \\
\hline April 2008 - Juli 2011 & $\begin{array}{l}\text { Doktorand im GAUSS-Programm } \\
\text { der Georg-August-Universität Göttingen }\end{array}$ \\
\hline Oktober 2003 - März 2008 & $\begin{array}{l}\text { Studium der Mathematik } \\
\text { an der Georg-August-Universität Göttingen } \\
\text { Abschluss: Diplom }\end{array}$ \\
\hline 1995 - Juni 2002 & $\begin{array}{l}\text { Gymnasiale Oberstufe } \\
\text { der Adam-von-Trott Schule Sontra } \\
\text { Schulabschluss: Abitur }\end{array}$ \\
\hline $1993-1995$ & Orientierungsstufe, Mittelpunktschule Wehretal \\
\hline $1989-1993$ & Grundschule Wehretal \\
\hline
\end{tabular}

